\title{
Synthesis of $(E)$-Quinoxalinone Oximes through a Multicomponent Reaction under Mild Conditions
}

Jun Xu, Huiyong Yang, Lei He, Lin Huang, Jiabin Shen, Wanmei Li* and Pengfei Zhang*

College of Material Chemistry and Chemical Engineering, Hangzhou Normal University, Hangzhou 311121, China.

Email: liwanmei@hznu.edu.cn (Li, W.); pfzhang@hznu.edu.cn (Zhang, P.)

\section{Supporting Information}

\section{Table of contents}

General Information

1. Optimization of Reaction Conditions 2

2. Experimental Section 3

3. Characterization of the Products 8

4. Computational Details 24

5. References 26

6. X-ray Crystal Data for $\mathbf{1 8}$ and $\mathbf{2 0} 27$

7. Copies of ${ }^{1} \mathrm{H},{ }^{13} \mathrm{C}$ and ${ }^{19} \mathrm{~F}$ NMR Spectra

8. Copies of HRMS Spectra 81 


\section{General Information}

All reagents and deuterated solvents were commercially available and used without further purification. All ketones (2) were purchased from Energy Chemical. All products were separated by silica gel (200-300 mesh) column chromatography with petroleum ether (PE) $\left(60-90{ }^{\circ} \mathrm{C}\right)$ and ethyl acetate (EA). ${ }^{1} \mathrm{H}$ and ${ }^{13} \mathrm{C}$ NMR spectra were recorded on Bruker Advance 500 or 400 spectrometers at ambient temperature with $\mathrm{CDCl}_{3}$ or $\mathrm{CD}_{3} \mathrm{SOCD}_{3}$ as solvent and tetramethylsilane (TMS) as the internal standard. Melting points were determined on an X-5 Data microscopic melting point apparatus. Analytical thin layer chromatography (TLC) was performed on Merk precoated TLC (silica gel 60 F254) plates. Compounds for HRMS were analyzed by positive mode electrospray ionization (ESI) using Agilent 6530 QTOF or Bruker solanX 70 FT-MS mass spectrometer.

\section{Optimization of Reaction Conditions ${ }^{a, b}$}

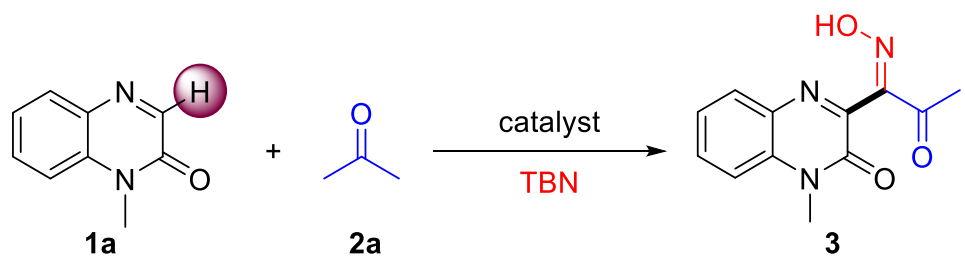

$\begin{array}{ccccc}\text { entry } & \text { catalyst } & \text { 2a (x quiv) } & \text { TBN (y quiv) } & \text { yield }(\%)^{b} \\ 1 & \mathrm{CH}_{3} \mathrm{CO}_{2} \mathrm{H} & 10 & 1.2 & \text { trace } \\ 2 & \mathrm{CF}_{3} \mathrm{CO}_{2} \mathrm{H} & 10 & 1.2 & 23 \\ 3 & \mathrm{CH}_{3} \mathrm{SO}_{3} \mathrm{H} & 10 & 1.2 & 57 \\ 4 & \mathrm{CF}_{3} \mathrm{SO}_{3} \mathrm{H} & 10 & 1.2 & 52 \\ 5 & \mathrm{H}_{2} \mathrm{SO}_{4} & 10 & 1.2 & 0 \\ 6 & \mathrm{Et}_{3} \mathrm{~N} & 10 & 1.2 & 0 \\ 7 & - & 10 & 1.2 & 0 \\ 8^{c} & \mathrm{CH}_{3} \mathrm{SO}_{3} \mathrm{H} & 10 & 1.2 & 81 \\ 9^{d} & \mathrm{CH}_{3} \mathrm{SO}_{3} \mathrm{H} & 10 & 1.2 & 79 \\ 10^{c} & \mathrm{CH}_{3} \mathrm{SO}_{3} \mathrm{H} & 3 & 1.2 & 76 \\ 11^{c} & \mathrm{CH}_{3} \mathrm{SO}_{3} \mathrm{H} & 5 & 1.2 & 82 \\ 12^{c} & \mathrm{CH}_{3} \mathrm{SO}_{3} \mathrm{H} & 20 & 1.2 & 83 \\ 13^{c} & \mathrm{CH}_{3} \mathrm{SO}_{3} \mathrm{H} & 5 & 1.0 & 76 \\ 14^{c} & \mathrm{CH}_{3} \mathrm{SO}_{3} \mathrm{H} & 5 & 1.5 & 82 \\ 15^{c} & \mathrm{CH}_{3} \mathrm{SO}_{3} \mathrm{H} & 5 & 2.0 & 78 \\ 16^{c, e} & \mathrm{CH}_{3} \mathrm{SO}_{3} \mathrm{H} & 5 & 1.2 & \text { trace } \\ 17^{c, f} & \mathrm{CH}_{3} \mathrm{SO}_{3} \mathrm{H} & 5 & 1.2 & 79 \\ 18^{c, g} & \mathrm{CH}_{3} \mathrm{SO}_{3} \mathrm{H} & 5 & 1.2 & 80\end{array}$

a Reaction conditions: 1a $(5.0 \mathrm{mmol})$, 2a (x equiv.), catalyst $(15 \mathrm{~mol} \%)$, NO source $(1.2$ equiv.), room temperature open flask, $6 \mathrm{~h}$. ${ }^{\mathrm{b}}$ Isolated yields by recrystallisation. ${ }^{\mathrm{c}} \mathrm{CH}_{3} \mathrm{SO}_{3} \mathrm{H}$ (25.0 mol\%). ${ }^{\mathrm{d}} \mathrm{CH}_{3} \mathrm{SO}_{3} \mathrm{H}(50.0 \mathrm{~mol} \%) .{ }^{\mathrm{e}}$ Under $\mathrm{N}_{2} .{ }^{\mathrm{f}}$ Extended reaction time to $12 \mathrm{~h}^{\mathrm{g}}{ }^{\mathrm{g}} \mathbf{1 a}(10.0$ mmol) was used. TBN = tert-butyl nitrite. 


\section{Experimental Section}

\subsection{Preparation of substrates 1}
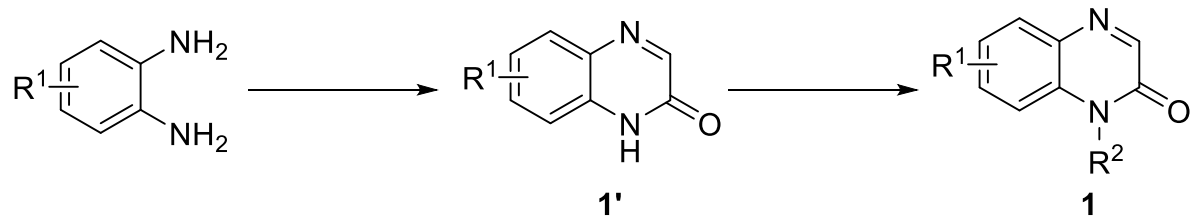

A mixture of $o$-phenylenediamine $(5 \mathrm{mmol})$, ethyl 2-oxoacetate $(6 \mathrm{mmol})$ and ethanol $(20 \mathrm{~mL})$ in a dried $50 \mathrm{~mL}$ round-bottom flask was stirred at reflux for 1 hour. After the completion (as indicated by TLC), the reaction mixture was filtered, washed with ethanol and then dried to give quinoxalinone 1'. Subsequently, A mixture of quinoxalinone 1', $\mathrm{K}_{2} \mathrm{CO}_{3}$ (1.2 equiv.), corresponding halogenoalkane (1.6 equiv.) and DMF $(20 \mathrm{ml})$ in a dried $50 \mathrm{~mL}$ round-bottom flask was stirred at room temperature overnight. After the completion (as indicated by TLC), The mixture was then extracted with ethyl acetate and the collected organic layer was washed with brine, dried with $\mathrm{MgSO}_{4}$. The solvent was removed under reduced pressure, and the crude product was further purified by silica gel column chromatography (200-300 mesh silica gel, $\mathrm{PE} / \mathrm{EA}=5: 1)$ to afford desired substrates $\mathbf{1}$. All the substrates $\mathbf{1}$ are known compounds. ${ }^{1}$

\subsection{General procedure for the synthesis of $(E)$-quinoxalinones oximes 3-39}

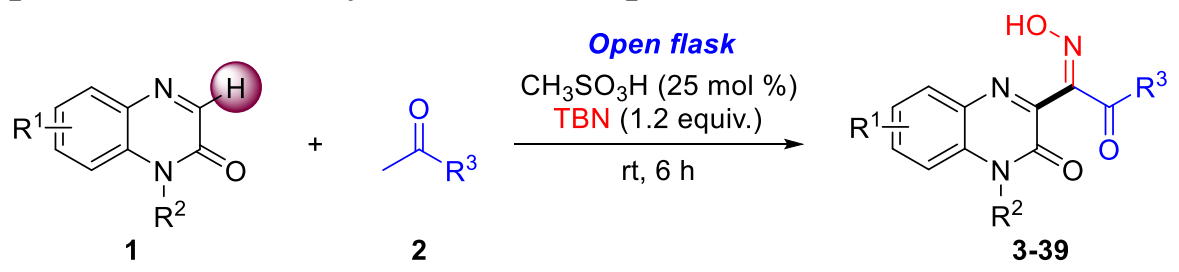

To a dried $25 \mathrm{~mL}$ tube was added quinoxalinones (1) (10.0 mmol), methyl ketones (2) (acetone (5.0 equiv.); other ketones (3.0 equiv.)) and $\mathrm{CH}_{3} \mathrm{SO}_{3} \mathrm{H}$ (25 mol\%). 1.2 equiv of TBN (as batches of 0.4 equiv. each with an interval of $2.0 \mathrm{~h}$ ) was then added to the above mixture under vigorous stirring at ambient temperature. After the completion (as indicated by TLC), the reaction was quenched with saturated $\mathrm{NaHCO}_{3}$. The crude product was obtained through filtration and dryness, which was further purified by recrystallisation using the mixture of ethyl acetate and petroleum ether.

\subsection{General procedure for the synthesis of $(E)$-quinoxalinone oxime 3 on a 1 mmol scale}

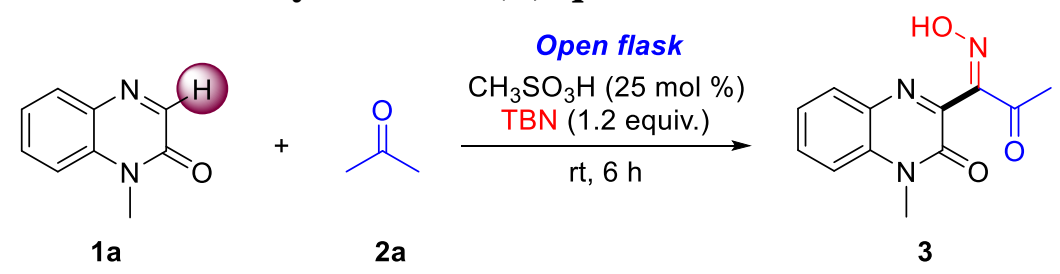

To a dried $5 \mathrm{~mL}$ tube was added quinoxalinones (1a) $(1.0 \mathrm{mmol})$, acetone (2a) (5.0 equiv.) and $\mathrm{CH}_{3} \mathrm{SO}_{3} \mathrm{H}(25 \mathrm{~mol} \%) .1 .2$ equiv of TBN (as batches of 0.4 equiv. each with an interval of $2.0 \mathrm{~h}$ ) was then added to the above mixture under vigorous stirring at ambient temperature. After the completion (as indicated by TLC), the reaction was quenched with saturated $\mathrm{NaHCO}_{3}$. The crude product was obtained through filtration and dryness, which was further purified by 
recrystallisation using the mixture of ethyl acetate and petroleum ether $(85 \%, 208 \mathrm{mg})$.

\subsection{General procedure for the synthesis of $(E)$-quinoxalinones oximes 40-43}

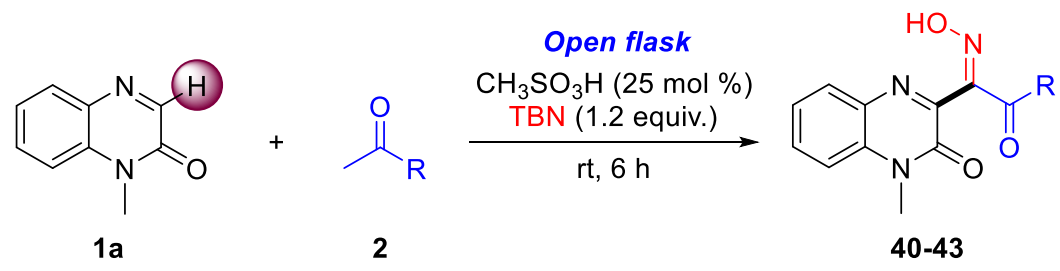

To a dried $15 \mathrm{~mL}$ tube was added quinoxalinone (1a) $(5.0 \mathrm{mmol})$, Raspberry ketone, Vanillylacetone, Nabumetone or 5-alpha-Dihydroprogesterone (2.0 equiv.), DCM (3.0 equiv.) and $\mathrm{CH}_{3} \mathrm{SO}_{3} \mathrm{H}(25 \mathrm{~mol} \%)$. 1.2 equiv of TBN (as batches of 0.4 equiv. each with an interval of $2.0 \mathrm{~h}$ ) was then added to the above mixture under vigorous stirring at ambient temperature. After the completion (as indicated by TLC), the reaction was quenched with saturated $\mathrm{NaHCO}_{3}$. The crude product was obtained through filtration and dryness, which was further purified by recrystallisation using the mixture of ethyl acetate and petroleum ether.

\subsection{General procedure for the synthesis of 44 using 3 as starting material}

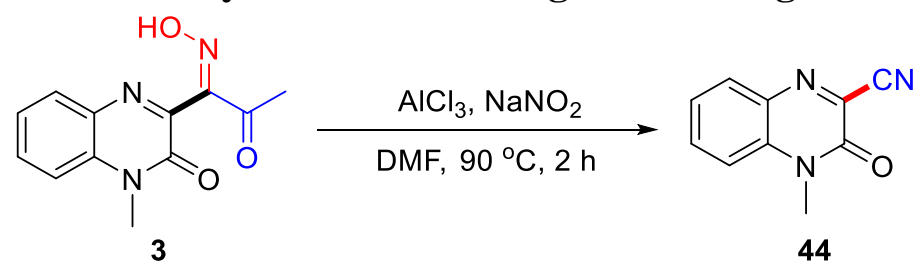

A mixture of (E)-quinoxalinones oxime 3 (2.0 mmol), $\mathrm{NaNO}_{2}$ (10.0 equiv.), $\mathrm{AlCl}_{3}$ (2.0 equiv.) and DMF $(5.0 \mathrm{~mL})$ in a $15 \mathrm{~mL}$ pressure tube was stirred at $90{ }^{\circ} \mathrm{C}$ for 2 hours. After the completion (as indicated by TLC), the reaction was quenched with saturated $\mathrm{NaHCO}_{3}$. The mixture was then extracted with ethyl acetate and the collected organic layer was washed with brine, dried with $\mathrm{MgSO}_{4}$. The solvent was removed under reduced pressure, and the crude product was further purified by silica gel column chromatography (200-300 mesh silica gel, $\mathrm{PE} / \mathrm{EA}=5: 1)$ to afford product 44.

\subsection{General procedure for the synthesis of 45 using 3 as starting material}

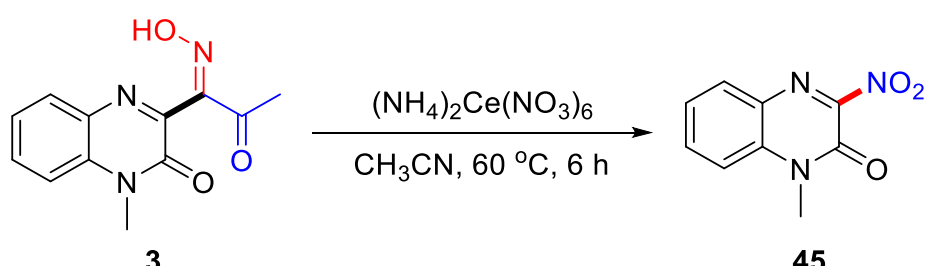

A mixture of $(E)$-quinoxalinones oxime $3(2.0 \mathrm{mmol}),\left(\mathrm{NH}_{4}\right)_{2} \mathrm{Ce}\left(\mathrm{NO}_{3}\right)_{6}$ (2.0 equiv.), $\mathrm{CH}_{3} \mathrm{CN}$ $(5.0 \mathrm{~mL})$ in a $15 \mathrm{~mL}$ tube was stirred at $60{ }^{\circ} \mathrm{C}$ for 6 hours. After the completion (as indicated by TLC), the reaction was quenched with saturated $\mathrm{NaHCO}_{3}$. The mixture was then extracted with ethyl acetate and the collected organic layer was washed with brine, dried with $\mathrm{MgSO}_{4}$. The solvent was removed under reduced pressure, and the crude product was further purified by silica gel column chromatography (200-300 mesh silica gel, PE/EA = 3:1) to afford product 45 . 


\subsection{General procedure for the synthesis of 46 using 3 as starting material}<smiles>CO/N=C(/C(C)=O)c1nc2ccccc2n(C)c1=O</smiles>

To a dried $25 \mathrm{~mL}$ tube was added $(E)$-quinoxalinones oxime $3(2.0 \mathrm{mmol})$ and DMF $(5.0 \mathrm{~mL})$. To this solution 1.5 equiv of $\mathrm{NaH}$ was added followed by the addition of 1.5 equiv. of $\mathrm{CH}_{3} \mathrm{I}$ under vigorous stirring at ambient temperature. After the completion (as indicated by TLC), the reaction was quenched with saturated $\mathrm{NaHCO}_{3}$. The mixture was then extracted with ethyl acetate and the collected organic layer was washed with brine, dried with $\mathrm{MgSO}_{4}$. The solvent was removed under reduced pressure, and the crude product was further purified by silica gel column chromatography (200-300 mesh silica gel, PE/EA = 5:1) to afford product 46.

\subsection{General procedure for radical inhibition experiment}

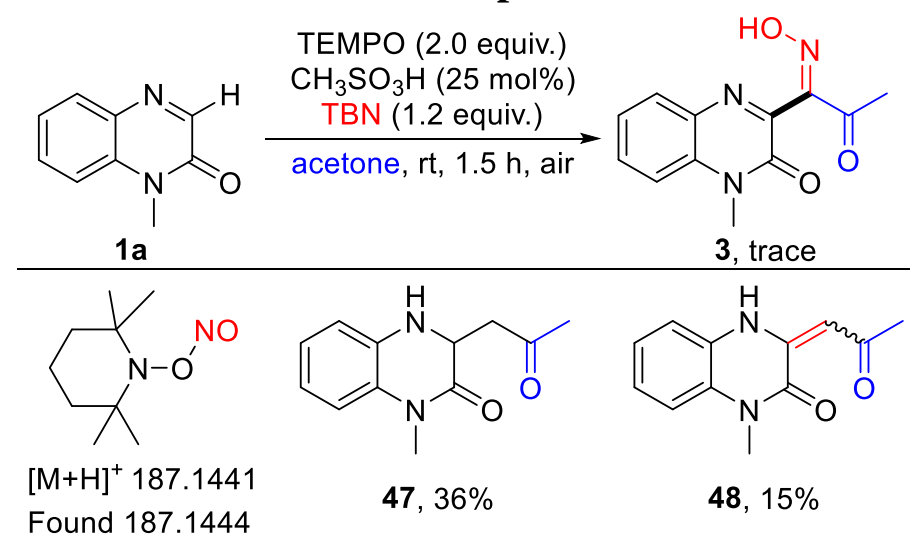

To a dried $10 \mathrm{~mL}$ tube was added quinoxalinones (1a) $(0.5 \mathrm{mmol})$, acetone $(0.5 \mathrm{~mL}), \mathrm{CH}_{3} \mathrm{SO}_{3} \mathrm{H}$ (25 mol\%) and TEMPO (2.0 equiv.). 1.2 equiv of TBN (as batches of 0.4 equiv. each with an interval of $0.5 \mathrm{~h}$ ) was then added to the above mixture under vigorous stirring at ambient temperature for $1.5 \mathrm{~h}$. Intermediate $\mathbf{4 7}$ and $\mathbf{4 8}$ were obtained instead of target product $\mathbf{3}$, and the NO radical was also captured. These experimental results clearly reveal that a radical mechanism is responsible for the reaction, but the generation of $\mathbf{4 7}$ and $\mathbf{4 8}$ may not involve radical pathway.

\subsection{General procedure for the synthesis of 48 using $1 \mathrm{a}$ as starting material}

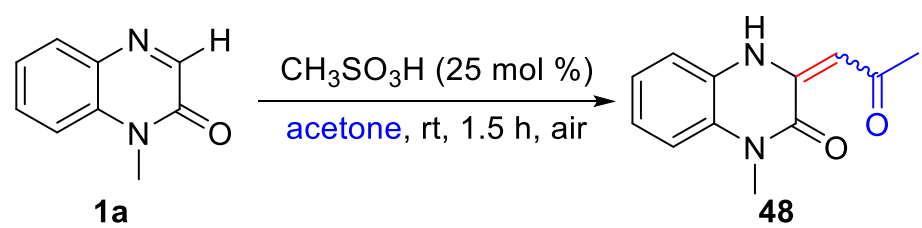

To a dried $10 \mathrm{~mL}$ tube was added quinoxalinones $(\mathbf{1 a})(0.5 \mathrm{mmol})$, acetone $(0.5 \mathrm{~mL})$ and $\mathrm{CH}_{3} \mathrm{SO}_{3} \mathrm{H}(25 \mathrm{~mol} \%)$. The mixture was stirred at ambient temperature for $1.5 \mathrm{~h}$. After the completion (as indicated by TLC), the reaction was quenched with saturated $\mathrm{NaHCO}_{3}$. The mixture was then extracted with ethyl acetate and the collected organic layer was washed with brine, dried with $\mathrm{MgSO}_{4}$. The solvent was removed under reduced pressure, and the crude 
product was further purified by silica gel column chromatography (200-300 mesh silica gel, $\mathrm{PE} / \mathrm{EA}=5: 1)$ to afford product 48.

2.10General procedure for the synthesis of $(E)$-quinoxalinones oxime 3 using 48 as starting material

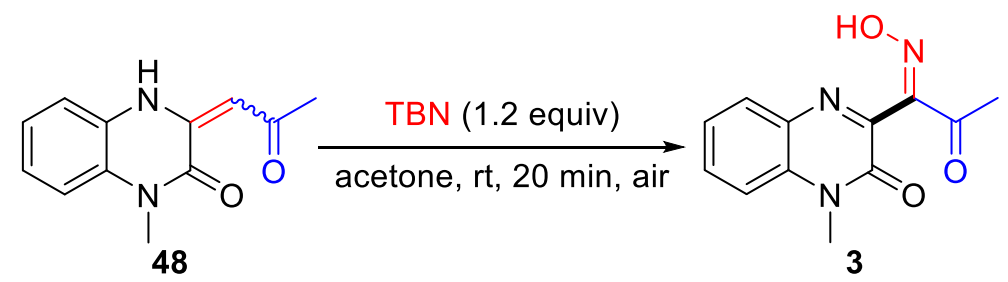

To a dried $10 \mathrm{~mL}$ tube was added compound (48) $(0.5 \mathrm{mmol})$, acetone $(\mathbf{2 a})(0.5 \mathrm{~mL})$ and TBN (1.2 equiv.). The mixture was stirred at ambient temperature for $20 \mathrm{~min}$. After the completion (as indicated by TLC), the reaction was quenched with saturated $\mathrm{NaHCO}_{3}$. The mixture was then extracted with ethyl acetate and the collected organic layer was washed with brine, dried with $\mathrm{MgSO}_{4}$. The solvent was removed under reduced pressure, and the crude product was further purified by silica gel column chromatography (200-300 mesh silica gel, PE/EA = 2:1) to afford product 3 .

\subsection{General procedure for the synthesis of 48 using 47 as starting material}

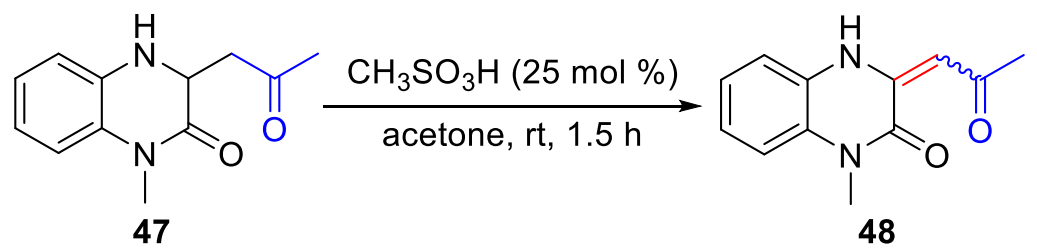

To a dried $10 \mathrm{~mL}$ tube was added compound (47) $(0.5 \mathrm{mmol})$, acetone $(0.5 \mathrm{~mL})$ and $\mathrm{CH}_{3} \mathrm{SO}_{3} \mathrm{H}$ (25 mol\%). The mixture was stirred at ambient temperature under specified atmosphere for 1.5 h. After the completion (as indicated by TLC), the reaction was quenched with saturated $\mathrm{NaHCO}_{3}$. The mixture was then extracted with ethyl acetate and the collected organic layer was washed with brine, dried with $\mathrm{MgSO}_{4}$. The solvent was removed under reduced pressure, and the crude product was further purified by silica gel column chromatography (200-300 mesh silica gel, PE/EA = 5:1) to afford product 48.

\subsection{General procedure for sample preparation and crystal measurement}

The single crystals of products $\mathbf{1 8}$ and $\mathbf{2 0}$ were prepared through volatilization method using the mixture dichloromethane and diethyl ether as solvent. Then, a suitable crystal was selected and placed on a CCD area detector diffractometer. The crystal was kept at corresponding temperature (100.15 K for 18 and $296.15 \mathrm{~K}$ for 20) during data collection. The structure was solved with the Olex2. Solve structure solution program using Charge Flipping and refined with the ShelXL refinement package using Least Squares minimisation. 


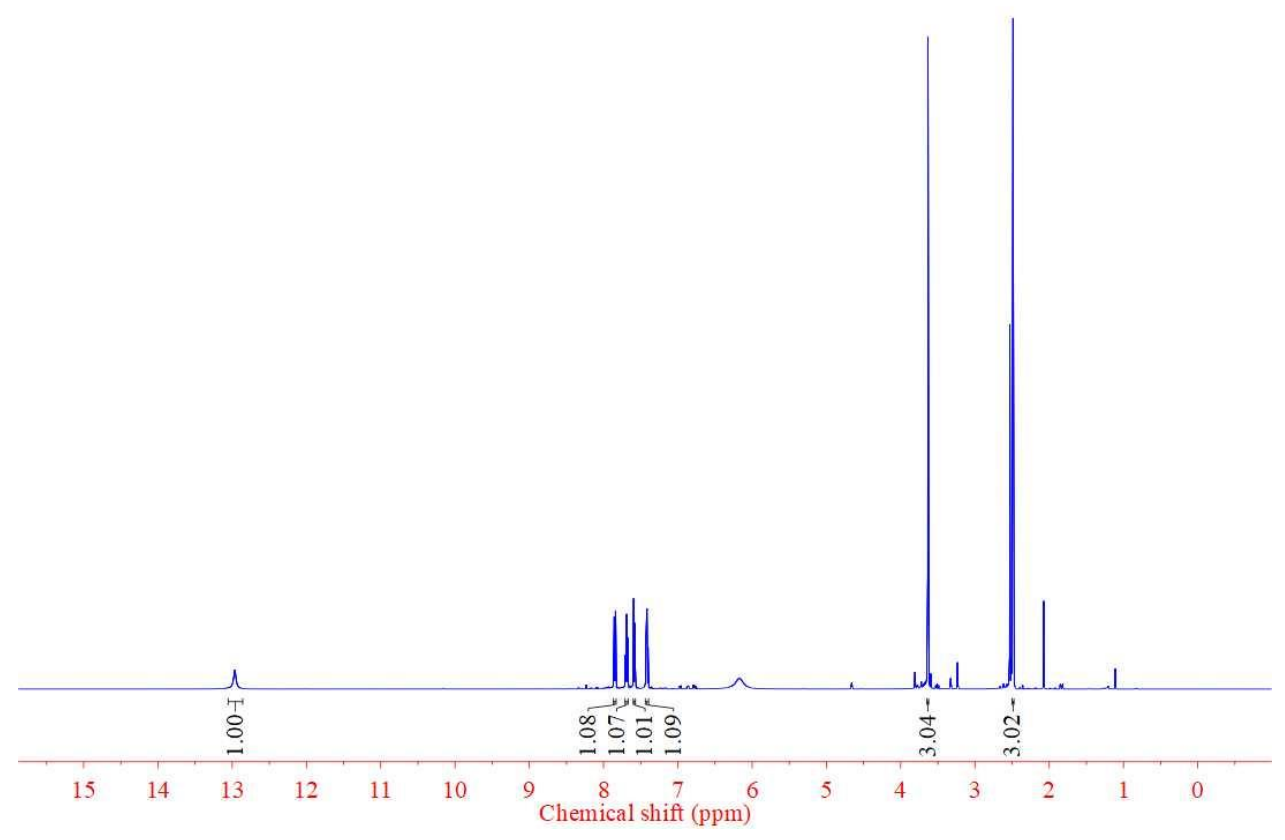

Figure S1 ${ }^{1} \mathrm{H}$ NMR spectrum of crude product 3

The NMR spectrum of crude product 3 has been recorded (Figure S1), this test proved that there was no other stereoisomer exists.

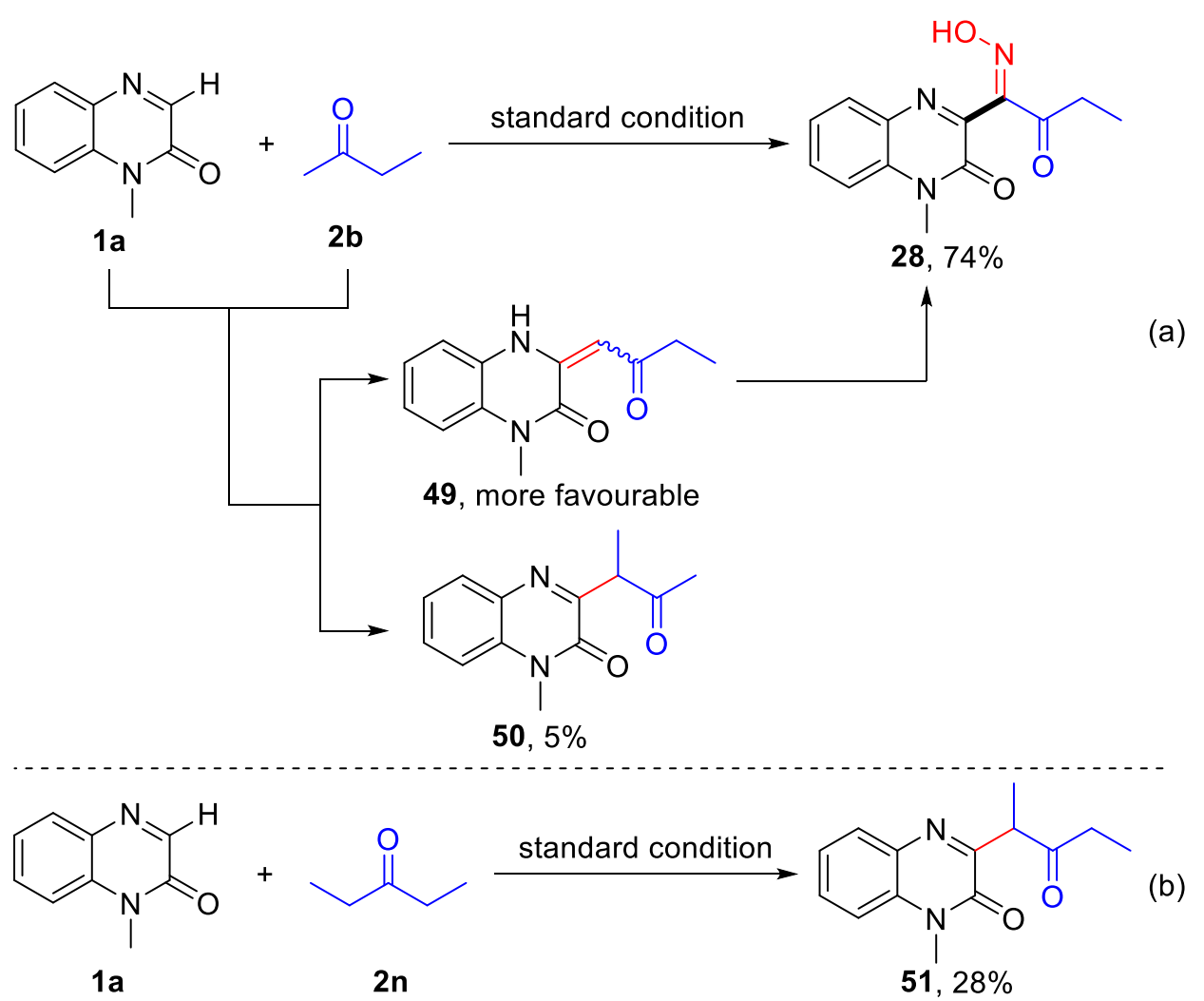

Scheme S1 The formation of compounds $\mathbf{5 0}$ and $\mathbf{5 1}$ 


\section{Characterization of the Products}

(E)-3-(1-(Hydroxyimino)-2-oxopropyl)-1-methylquinoxalin-2(1H)-one (3)<smiles>CC(=O)/C(=N/O)c1nc2ccccc2n(C)c1=O</smiles>

Purified by recrystallisation using the mixture of ethyl acetate and petroleum ether as solvent. Brown solid (1.96 g, 80\% yield); M.p. 223-224 ${ }^{\circ} \mathrm{C} .{ }^{1} \mathrm{H}$ NMR (500 MHz, DMSO) $\delta 12.94$ (s, $1 \mathrm{H}), 7.86(\mathrm{~d}, J=7.5 \mathrm{~Hz}, 1 \mathrm{H}), 7.72(\mathrm{~s}, 1 \mathrm{H}), 7.62(\mathrm{~d}, J=6.8 \mathrm{~Hz}, 1 \mathrm{H}), 7.44(\mathrm{~d}, J=6.4 \mathrm{~Hz}, 1 \mathrm{H})$, 3.65 (s, 3H), 2.49 (s, 3H); ${ }^{13} \mathrm{C}$ NMR (126 MHz, DMSO) $\delta$ 195.9, 153.5, 152.7, 152.4, 133.6, 132.3, 132.1, 130.1, 124.4, 115.6, 29.4, 25.9; HRMS (ESI) m/z: $[\mathrm{M}+\mathrm{Na}]^{+}$Calcd for $\mathrm{C}_{12} \mathrm{H}_{11} \mathrm{~N}_{3} \mathrm{O}_{3} \mathrm{Na}$ 268.0693; Found 268.0696.

(E)-1-Ethyl-3-(1-(hydroxyimino)-2-oxopropyl)quinoxalin-2(1H)-one (4)<smiles>CCn1c(=O)c(/C(=N\O)C(C)=O)nc2ccccc21</smiles>

Purified by recrystallisation using the mixture of ethyl acetate and petroleum ether as solvent. Brown solid (2.02 g, 78\% yield); M.p. $216-217{ }^{\circ} \mathrm{C} .{ }^{1} \mathrm{H}$ NMR (500 MHz, DMSO) $\delta 12.94$ (s, $1 \mathrm{H}), 7.88(\mathrm{~d}, J=7.8 \mathrm{~Hz}, 1 \mathrm{H}), 7.75-7.69(\mathrm{~m}, 2 \mathrm{H}), 7.44(\mathrm{t}, J=6.6 \mathrm{~Hz}, 1 \mathrm{H}), 4.28(\mathrm{q}, J=7.0 \mathrm{~Hz}$, 2H), 2.49 (s, 3H), 1.25 (t, $J=7.1 \mathrm{~Hz}, 3 \mathrm{H}) ;{ }^{13} \mathrm{C}$ NMR (126 MHz, DMSO) $\delta 195.9,153.4,152.4$, $152.3,132.6,132.4,132.2,130.5,124.3,115.3,37.4,25.9,12.8 ;$ HRMS (ESI) m/z: $[\mathrm{M}+\mathrm{Na}]^{+}$ Calcd for $\mathrm{C}_{13} \mathrm{H}_{13} \mathrm{~N}_{3} \mathrm{O}_{3} \mathrm{Na} 282.0849$; Found 282.0850.

(E)-1-Butyl-3-(1-(hydroxyimino)-2-oxopropyl)quinoxalin-2(1H)-one (5)<smiles>CCCCn1c(=O)c(/C(=N\O)C(C)=O)nc2ccccc21</smiles>

Purified by recrystallisation using the mixture of ethyl acetate and petroleum ether as solvent. Brown solid (2.33 g, 81\% yield); M.p. $229-230{ }^{\circ} \mathrm{C} .{ }^{1} \mathrm{H}$ NMR (500 MHz, DMSO) $\delta 12.91$ (s, $1 \mathrm{H}), 7.87(\mathrm{~d}, J=6.8 \mathrm{~Hz}, 1 \mathrm{H}), 7.74-7.67(\mathrm{~m}, 2 \mathrm{H}), 7.43(\mathrm{t}, J=8.1 \mathrm{~Hz}, 1 \mathrm{H}), 4.26-4.21(\mathrm{~m}$, 2H), $2.48(\mathrm{~s}, 3 \mathrm{H}), 1.63(\mathrm{dd}, J=15.0,7.3 \mathrm{~Hz}, 2 \mathrm{H}), 1.37(\mathrm{dd}, J=15.0,7.4 \mathrm{~Hz}, 2 \mathrm{H}), 0.92(\mathrm{t}, J=$ $7.4 \mathrm{~Hz}, 3 \mathrm{H}) ;{ }^{13} \mathrm{C}$ NMR (126 MHz, DMSO) $\delta 195.8,153.5,152.6,152.4,132.6,132.6,132.2$, 130.4, 124.3, 115.4, 41.8, 29.5, 25.9, 20.0, 14.1; HRMS (ESI) $\mathrm{m} / \mathrm{z}:[\mathrm{M}+\mathrm{Na}]^{+} \mathrm{Calcd}$ for $\mathrm{C}_{15} \mathrm{H}_{17} \mathrm{~N}_{3} \mathrm{O}_{3} \mathrm{Na}$ 310.1162; Found 310.1162. 
(E)-3-(1-(Hydroxyimino)-2-oxopropyl)-1-isobutylquinoxalin-2(1H)-one (6)<smiles>CC(=O)/C(=N/O)c1nc2ccccc2n(CC(C)C)c1=O</smiles>

Purified by recrystallisation using the mixture of ethyl acetate and petroleum ether as solvent. Brown solid (2.21 g, 77\% yield); M.p. 222-223 ${ }^{\circ} \mathrm{C} .{ }^{1} \mathrm{H}$ NMR (500 MHz, DMSO) $\delta 12.92$ (s, $1 \mathrm{H}), 7.85(\mathrm{~d}, J=7.8 \mathrm{~Hz}, 1 \mathrm{H}), 7.67(\mathrm{q}, J=8.0 \mathrm{~Hz}, 2 \mathrm{H}), 7.41(\mathrm{t}, J=6.9 \mathrm{~Hz}, 1 \mathrm{H}), 4.12(\mathrm{~d}, J=7.1$ $\mathrm{Hz}, 2 \mathrm{H}), 2.48(\mathrm{~s}, 3 \mathrm{H}), 2.17-2.08(\mathrm{~m}, 1 \mathrm{H}), 0.89(\mathrm{~d}, J=6.3 \mathrm{~Hz}, 6 \mathrm{H}) ;{ }^{13} \mathrm{C}$ NMR $(126 \mathrm{MHz}$, DMSO) $\delta 195.8,153.5,153.0,152.5,132.8,132.5,132.0,130.4,124.2,115.8,48.3,27.2,25.9$, 20.2; HRMS (ESI) m/z: [M+Na $]^{+}$Calcd for $\mathrm{C}_{15} \mathrm{H}_{17} \mathrm{~N}_{3} \mathrm{O}_{3} \mathrm{Na}$ 310.1162; Found 310.1165.

\section{(E)-3-(1-(Hydroxyimino)-2-oxopropyl)-1-isopentylquinoxalin-2(1H)-one (7)}<smiles>CC(=O)/C(=N/O)c1nc2ccccc2n(CCC(C)(C)C)c1=O</smiles>

Purified by recrystallisation using the mixture of ethyl acetate and petroleum ether as solvent. Brown solid (2.26 g, 75\% yield); M.p. $234-23{ }^{\circ} \mathrm{C} .{ }^{1} \mathrm{H}$ NMR (500 MHz, DMSO) $\delta 12.93$ (s, 1H), $7.87(\mathrm{~d}, J=7.4 \mathrm{~Hz}, 1 \mathrm{H}), 7.73(\mathrm{t}, J=7.4 \mathrm{~Hz}, 1 \mathrm{H}), 7.63(\mathrm{~d}, J=8.5 \mathrm{~Hz}, 1 \mathrm{H}), 7.43(\mathrm{t}, J=7.5 \mathrm{~Hz}$, $1 \mathrm{H}), 4.30-4.19(\mathrm{~m}, 2 \mathrm{H}), 2.49$ (s, 3H), 1.69 (td, $J=13.1,6.5 \mathrm{~Hz}, 1 \mathrm{H}), 1.51$ (dd, $J=15.1,7.0$ $\mathrm{Hz}, 2 \mathrm{H}), 0.96(\mathrm{~d}, J=6.6 \mathrm{~Hz}, 6 \mathrm{H}) ;{ }^{13} \mathrm{C} \mathrm{NMR}(126 \mathrm{MHz}, \mathrm{DMSO}) \delta 195.8,153.4,152.5,152.3$, 132.6, 132.5, 132.2, 130.5, 124.3, 115.3, 36.1, 26.1, 25.9, 22.8; HRMS (ESI) m/z: $[\mathrm{M}+\mathrm{Na}]^{+}$ Calcd for $\mathrm{C}_{16} \mathrm{H}_{19} \mathrm{~N}_{3} \mathrm{O}_{3} \mathrm{Na}$ 324.1319; Found 324.1317.

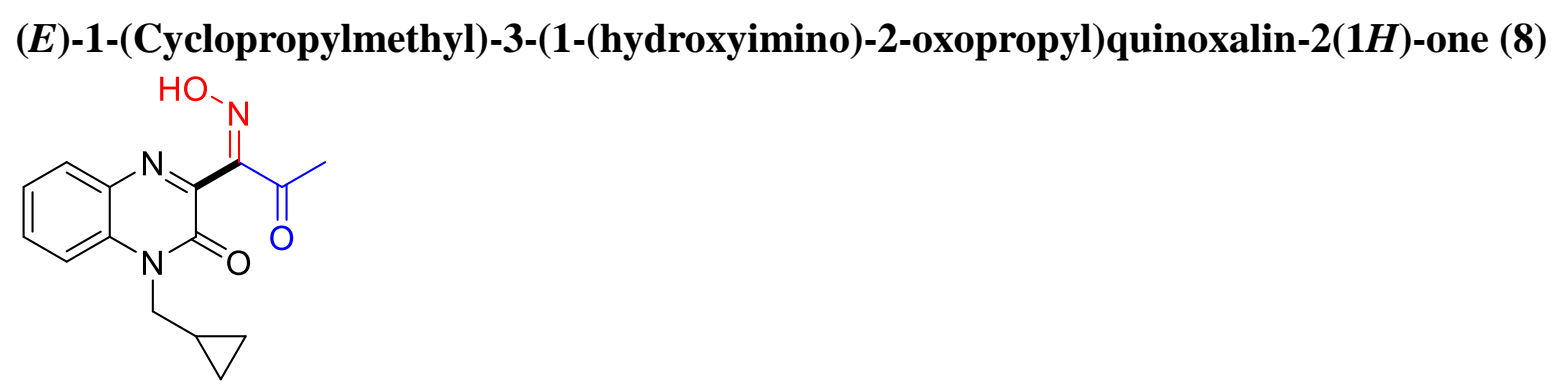

Purified by recrystallisation using the mixture of ethyl acetate and petroleum ether as solvent. Brown solid (2.02 g, 71\% yield); M.p. 202-203 ${ }^{\circ} \mathrm{C} .{ }^{1} \mathrm{H}$ NMR (500 MHz, DMSO) $\delta 12.92$ (s, $1 \mathrm{H}), 7.88(\mathrm{~d}, J=7.7 \mathrm{~Hz}, 1 \mathrm{H}), 7.79(\mathrm{~d}, J=8.4 \mathrm{~Hz}, 1 \mathrm{H}), 7.73(\mathrm{t}, J=7.6 \mathrm{~Hz}, 1 \mathrm{H}), 7.44(\mathrm{t}, J=7.5$ $\mathrm{Hz}, 1 \mathrm{H}), 4.19(\mathrm{~d}, J=6.9 \mathrm{~Hz}, 2 \mathrm{H}), 2.48(\mathrm{~s}, 3 \mathrm{H}), 1.27(\mathrm{dd}, J=11.0,6.6 \mathrm{~Hz}, 1 \mathrm{H}), 0.52-0.43(\mathrm{~m}$, $4 \mathrm{H}) ;{ }^{13} \mathrm{C}$ NMR (126 MHz, DMSO) $\delta$ 195.9, 153.5, 152.9, 152.5, 132.9, 132.5, 132.1, 130.4, 124.3, 115.7, 45.9, 25.9, 10.0, 4.2; HRMS (ESI) m/z: $[\mathrm{M}+\mathrm{Na}]^{+}$Calcd for $\mathrm{C}_{15} \mathrm{H}_{15} \mathrm{~N}_{3} \mathrm{O}_{3} \mathrm{Na}$ 308.1006; Found 308.1006. 
<smiles>CC(=O)/C(=N/O)c1nc2ccccc2n(CC2CCCCC2)c1=O</smiles>

Purified by recrystallisation using the mixture of ethyl acetate and petroleum ether as solvent. Brown solid (2.22 g, 68\% yield); M.p. $229-230{ }^{\circ} \mathrm{C} .{ }^{1} \mathrm{H}$ NMR (500 MHz, DMSO) $\delta 12.93$ (s, $1 \mathrm{H}), 7.86(\mathrm{~d}, J=7.8 \mathrm{~Hz}, 1 \mathrm{H}), 7.73-7.62(\mathrm{~m}, 2 \mathrm{H}), 7.42(\mathrm{t}, J=7.4 \mathrm{~Hz}, 1 \mathrm{H}), 4.14(\mathrm{~d}, J=7.1 \mathrm{~Hz}$, 2H), $2.50(\mathrm{~s}, 3 \mathrm{H}), 1.81(\mathrm{~s}, 1 \mathrm{H}), 1.64-1.57(\mathrm{~m}, 5 \mathrm{H}), 1.19-1.06(\mathrm{~m}, 5 \mathrm{H}) ;{ }^{13} \mathrm{C} \mathrm{NMR}(126 \mathrm{MHz}$, DMSO) $\delta$ 195.8, 153.5, 153.0, 152.4, 132.9, 132.6, 132.0, 130.4, 124.2, 115.7, 47.3, 36.4, 30.5, 26.2, 25.9, 25.7; HRMS (ESI) m/z: $[\mathrm{M}+\mathrm{Na}]^{+}$Calcd for $\mathrm{C}_{18} \mathrm{H}_{21} \mathrm{~N}_{3} \mathrm{O}_{3} \mathrm{Na}$ 350.1475; Found 350.1482 .

Methyl (E)-2-(3-(1-(hydroxyimino)-2-oxopropyl)-2-oxoquinoxalin-1(2H)-yl)acetate (10)<smiles>COC(=O)Cn1c(=O)c(/C(=N\O)C(C)=O)nc2ccccc21</smiles>

Purified by recrystallisation using the mixture of ethyl acetate and petroleum ether as solvent. Brown solid (2.21 g, 73\% yield); M.p. 220-221 ${ }^{\circ} \mathrm{C} .{ }^{1} \mathrm{H}$ NMR (500 MHz, DMSO) $\delta 12.99$ (s, $1 \mathrm{H}), 7.90(\mathrm{~d}, J=9.3 \mathrm{~Hz}, 1 \mathrm{H}), 7.70(\mathrm{t}, J=8.6 \mathrm{~Hz}, 1 \mathrm{H}), 7.59$ (d, $J=8.1 \mathrm{~Hz}, 1 \mathrm{H}), 7.46$ (t, $J=7.1$ $\mathrm{Hz}, 1 \mathrm{H}), 5.14$ (s, 2H), 3.72 (s, 3H), 2.48 (s, 3H); ${ }^{13} \mathrm{C}$ NMR (126 MHz, DMSO) $\delta$ 195.8, 168.2, 153.0, 152.5, 152.2, 132.9, 132.4, 132.3, 130.4, 124.7, 115.5, 53.0, 43.9, 25.9; HRMS (ESI) $\mathrm{m} / \mathrm{z}:[\mathrm{M}+\mathrm{Na}]^{+}$Calcd for $\mathrm{C}_{14} \mathrm{H}_{13} \mathrm{~N}_{3} \mathrm{O}_{5} \mathrm{Na} 326.0747$; Found 326.0746.

(E)-1-Allyl-3-(1-(hydroxyimino)-2-oxopropyl)quinoxalin-2(1H)-one (11)<smiles>C=CCn1c(=O)c(/C(=N\O)C(C)=O)nc2ccccc21</smiles>

Purified by recrystallisation using the mixture of ethyl acetate and petroleum ether as solvent. Brown solid (1.82 g, 67\% yield); M.p. 200-201 ${ }^{\circ} \mathrm{C} .{ }^{1} \mathrm{H}$ NMR (500 MHz, DMSO) $\delta 13.03$ (s, $1 \mathrm{H}), 7.88(\mathrm{dd}, J=8.0,1.3 \mathrm{~Hz}, 1 \mathrm{H}), 7.72-7.67(\mathrm{~m}, 1 \mathrm{H}), 7.57(\mathrm{~d}, J=8.4 \mathrm{~Hz}, 1 \mathrm{H}), 7.43(\mathrm{t}, J=$ $7.5 \mathrm{~Hz}, 1 \mathrm{H}), 5.94(\mathrm{ddd}, J=22.2,10.1,4.9 \mathrm{~Hz}, 1 \mathrm{H}), 5.21(\mathrm{dd}, J=10.4,1.0 \mathrm{~Hz}, 1 \mathrm{H}), 5.03$ (dd, $J$ $=17.3,1.0 \mathrm{~Hz}, 1 \mathrm{H}), 4.89(\mathrm{~d}, J=4.8 \mathrm{~Hz}, 2 \mathrm{H}), 2.48(\mathrm{~s}, 3 \mathrm{H}) ;{ }^{13} \mathrm{C}$ NMR $(126 \mathrm{MHz}, \mathrm{DMSO}) \delta$ 
195.9, 153.4, 152.5, 152.4, 132.6, 132.5, 132.0, 131.6, 130.3, 124.4, 117.8, 115.8, 44.1, 25.9;

HRMS (ESI) m/z: [M+Na] ${ }^{+}$Calcd for $\mathrm{C}_{14} \mathrm{H}_{13} \mathrm{~N}_{3} \mathrm{O}_{3} \mathrm{Na}$ 294.0849; Found 294.0850.

(E)-3-(1-(Hydroxyimino)-2-oxopropyl)-1-(prop-2-yn-1-yl)quinoxalin-2(1H)-one (12)<smiles>C#CCn1c(=O)c(/C(=N\O)C(C)=O)nc2ccccc21</smiles>

Purified by recrystallisation using the mixture of ethyl acetate and petroleum ether as solvent. Brown solid (1.86 g, 69\% yield); M.p. $193-194{ }^{\circ} \mathrm{C} .{ }^{1} \mathrm{H}$ NMR (500 MHz, DMSO) $\delta 7.90$ (dd, $J$ $=8.0,1.3 \mathrm{~Hz}, 1 \mathrm{H}), 7.77(\mathrm{ddd}, J=8.6,7.3,1.4 \mathrm{~Hz}, 1 \mathrm{H}), 7.68(\mathrm{~d}, J=7.9 \mathrm{~Hz}, 1 \mathrm{H}), 7.51-7.45$ $(\mathrm{m}, 1 \mathrm{H}), 5.11(\mathrm{~d}, J=2.4 \mathrm{~Hz}, 2 \mathrm{H}), 3.39(\mathrm{t}, J=2.4 \mathrm{~Hz}, 1 \mathrm{H}), 2.48(\mathrm{~s}, 3 \mathrm{H}) ;{ }^{13} \mathrm{C} \mathrm{NMR}(126 \mathrm{MHz}$, DMSO) $\delta 200.7,157.9,157.7,156.6,137.3,136.9,136.6,135.1,129.6,120.5,82.9,80.8,36.5$, 30.6; HRMS (ESI) m/z: [M+Na] ${ }^{+}$Calcd for $\mathrm{C}_{14} \mathrm{H}_{11} \mathrm{~N}_{3} \mathrm{O}_{3} \mathrm{Na}$ 292.0693; Found 292.0693.

(E)-3-(1-(Hydroxyimino)-2-oxopropyl)-1-(2-methylbenzyl)quinoxalin-2(1H)-one (13)<smiles>CC(=O)/C(=N/O)c1nc2ccccc2n(Cc2ccccc2C)c1=O</smiles>

Purified by recrystallisation using the mixture of ethyl acetate and petroleum ether as solvent. Brown solid (2.18 g, 65\% yield); M.p. 246-247 ${ }^{\circ} \mathrm{C} .{ }^{1} \mathrm{H}$ NMR (500 MHz, DMSO) $\delta 13.04$ (s, $1 \mathrm{H}), 7.91(\mathrm{dd}, J=8.0,1.4 \mathrm{~Hz}, 1 \mathrm{H}), 7.60(\mathrm{ddd}, J=8.6,7.3,1.5 \mathrm{~Hz}, 1 \mathrm{H}), 7.45-7.39(\mathrm{~m}, 1 \mathrm{H})$, $7.28(\mathrm{dd}, J=14.4,7.7 \mathrm{~Hz}, 2 \mathrm{H}), 7.16(\mathrm{t}, J=7.4 \mathrm{~Hz}, 1 \mathrm{H}), 7.03(\mathrm{t}, J=7.4 \mathrm{~Hz}, 1 \mathrm{H}), 6.42(\mathrm{~d}, J=$ $7.7 \mathrm{~Hz}, 1 \mathrm{H}), 5.44$ (s, 2H), 2.49 (s, 3H), 2.44 (s, 3H); ${ }^{13} \mathrm{C}$ NMR (126 MHz, DMSO) $\delta 195.9$, 153.4, 153.0, 152.6, 138.5, 135.9, 132.6, 132.1, 130.4, 129.2, 128.7, 127.8, 124.6, 124.2, 115.9, 45.1, 25.9, 21.5; HRMS (ESI) m/z: $[\mathrm{M}+\mathrm{Na}]^{+}$Calcd for $\mathrm{C}_{19} \mathrm{H}_{17} \mathrm{~N}_{3} \mathrm{O}_{3} \mathrm{Na}$ 358.1162; Found 358.1163 .

(E)-1-(2-Fluorobenzyl)-3-(1-(hydroxyimino)-2-oxopropyl)quinoxalin-2(1H)-one (14)<smiles>CC(=O)/C(=N/O)c1nc2ccccc2n(Cc2ccccc2F)c1=O</smiles>

Purified by recrystallisation using the mixture of ethyl acetate and petroleum ether as solvent. Brown solid (2.03 g, 60\% yield); M.p. 206-207 ${ }^{\circ} \mathrm{C} .{ }^{1} \mathrm{H}$ NMR (500 MHz, DMSO) $\delta 13.08$ (s, 
1H), $7.91(\mathrm{dd}, J=8.0,1.4 \mathrm{~Hz}, 1 \mathrm{H}), 7.68-7.63(\mathrm{~m}, 1 \mathrm{H}), 7.48(\mathrm{~d}, J=7.9 \mathrm{~Hz}, 1 \mathrm{H}), 7.43(\mathrm{t}, J=$ $7.6 \mathrm{~Hz}, 1 \mathrm{H}), 7.35(\mathrm{dd}, J=13.3,6.2 \mathrm{~Hz}, 1 \mathrm{H}), 7.30-7.26(\mathrm{~m}, 1 \mathrm{H}), 7.12(\mathrm{t}, J=7.5 \mathrm{~Hz}, 1 \mathrm{H}), 6.89$ $(\mathrm{t}, J=7.7 \mathrm{~Hz}, 1 \mathrm{H}), 5.55(\mathrm{~s}, 2 \mathrm{H}), 2.50(\mathrm{~s}, 3 \mathrm{H}) ;{ }^{13} \mathrm{C}$ NMR (126 MHz, DMSO) $\delta 195.9,160.4$ $\left(\mathrm{C}-\mathrm{F}, 1 J_{\mathrm{C}-\mathrm{F}}=245.7 \mathrm{~Hz}\right), 153.3,152.9,152.5,132.6,132.3,130.5,130.2\left(\mathrm{C}-\mathrm{F}, 3 J_{\mathrm{C}-\mathrm{F}}=8.8 \mathrm{~Hz}\right)$, $128.2\left(\mathrm{C}-\mathrm{F}, 4 J_{\mathrm{C}-\mathrm{F}}=3.8 \mathrm{~Hz}\right), 125.3\left(\mathrm{C}-\mathrm{F}, 4 J_{\mathrm{C}-\mathrm{F}}=3.8 \mathrm{~Hz}\right), 124.7,122.8,122.7,116.2\left(\mathrm{C}-\mathrm{F}, 2 J_{\mathrm{C}-\mathrm{F}}\right.$ $=20.2 \mathrm{~Hz}), 115.4,39.7\left(\mathrm{C}-\mathrm{F}, 3 J_{\mathrm{C}-\mathrm{F}}=5.0 \mathrm{~Hz}\right), 25.9 ;{ }^{19} \mathrm{~F}$ NMR (471 MHz, DMSO) $\delta$-117.5; HRMS (ESI) m/z: [M+Na] ${ }^{+}$Calcd for $\mathrm{C}_{18} \mathrm{H}_{14} \mathrm{FN}_{3} \mathrm{O}_{3} \mathrm{Na} 362.0911$; Found 362.0933.

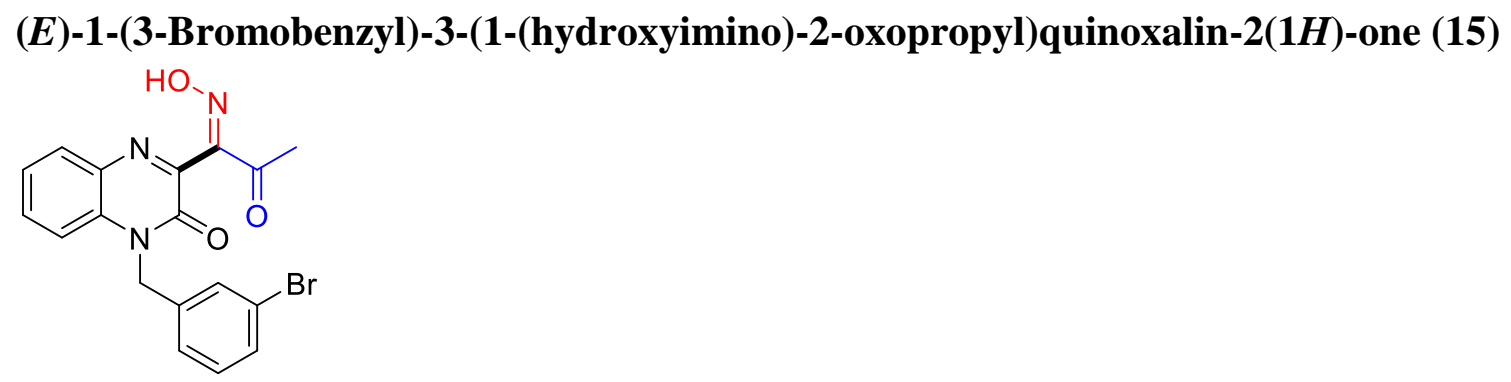

Purified by recrystallisation using the mixture of ethyl acetate and petroleum ether as solvent. Brown solid (2.19 g, 55\% yield); M.p. $251-252{ }^{\circ} \mathrm{C} .{ }^{1} \mathrm{H}$ NMR (500 MHz, DMSO) $\delta 13.04$ (s, $1 \mathrm{H}), 7.90(\mathrm{dd}, J=8.0,1.1 \mathrm{~Hz}, 1 \mathrm{H}), 7.69-7.63(\mathrm{~m}, 1 \mathrm{H}), 7.58(\mathrm{~d}, J=8.3 \mathrm{~Hz}, 1 \mathrm{H}), 7.48(\mathrm{~d}, J=$ $8.7 \mathrm{~Hz}, 2 \mathrm{H}), 7.43(\mathrm{t}, J=7.4 \mathrm{~Hz}, 1 \mathrm{H}), 7.31(\mathrm{t}, J=7.8 \mathrm{~Hz}, 1 \mathrm{H}), 7.21(\mathrm{~d}, J=7.7 \mathrm{~Hz}, 1 \mathrm{H}), 5.53(\mathrm{~s}$, 2H), 2.51 (s, 3H); ${ }^{13} \mathrm{C}$ NMR (101 MHz, DMSO) $\delta 195.4,152.8,152.4,152.0,138.3,132.1$, 132.0, 131.8, 131.0, 130.5, 130.0, 129.7, 125.7, 124.2, 122.0, 115.2, 44.1, 25.4; HRMS (ESI) $\mathrm{m} / \mathrm{z}:[\mathrm{M}+\mathrm{Na}]^{+} \mathrm{Calcd}$ for $\mathrm{C}_{18} \mathrm{H}_{14} \mathrm{BrN}_{3} \mathrm{O}_{3} \mathrm{Na} 422.0111$; Found 422.0113 .

\section{(E)-3-(1-(Hydroxyimino)-2-oxopropyl)-1-(4-methylbenzyl)quinoxalin-2(1H)-one (16)}<smiles>CC(=O)/C(=N/O)c1nc2ccccc2n(Cc2ccc(C)cc2)c1=O</smiles>

Purified by recrystallisation using the mixture of ethyl acetate and petroleum ether as solvent. Brown solid (2.31 g, 69\% yield); M.p. $237-238{ }^{\circ} \mathrm{C} .{ }^{1} \mathrm{H}$ NMR (500 MHz, DMSO) $\delta 12.99$ (s, $1 \mathrm{H}), 7.87(\mathrm{~d}, J=7.7 \mathrm{~Hz}, 1 \mathrm{H}), 7.62(\mathrm{t}, J=7.5 \mathrm{~Hz}, 1 \mathrm{H}), 7.55(\mathrm{~d}, J=8.4 \mathrm{~Hz}, 1 \mathrm{H}), 7.40(\mathrm{t}, J=7.5$ $\mathrm{Hz}, 1 \mathrm{H}), 7.14$ (s, 4H), 5.47 (s, 2H), 2.50 (s, 3H), 2.24 (s, 3H); ${ }^{13} \mathrm{C}$ NMR (126 MHz, DMSO) $\delta$ 195.9, 153.4, 153.0, 152.6, 137.3, 133.0, 132.6, 132.6, 132.1, 130.4, 129.8, 127.3, 124.5, 115.9, 44.9, 25.9, 21.1; HRMS (ESI) m/z: $[\mathrm{M}+\mathrm{Na}]^{+}$Calcd for $\mathrm{C}_{19} \mathrm{H}_{17} \mathrm{~N}_{3} \mathrm{O}_{3} \mathrm{Na}$ 358.1162; Found 358.1163. 
<smiles>CC(=O)/C(=N/O)c1nc2ccccc2n(Cc2ccc(F)cc2)c1=O</smiles>

Purified by recrystallisation using the mixture of ethyl acetate and petroleum ether as solvent. Brown solid (2.03 g, 60\% yield); M.p. $197-198{ }^{\circ} \mathrm{C} .{ }^{1} \mathrm{H}$ NMR (500 MHz, DMSO) $\delta 13.05$ (s, $1 \mathrm{H}), 7.90(\mathrm{~d}, J=7.0 \mathrm{~Hz}, 1 \mathrm{H}), 7.65(\mathrm{t}, J=7.2 \mathrm{~Hz}, 1 \mathrm{H}), 7.60(\mathrm{~d}, J=8.1 \mathrm{~Hz}, 1 \mathrm{H}), 7.42(\mathrm{t}, J=7.2$ $\mathrm{Hz}, 1 \mathrm{H}), 7.33(\mathrm{dd}, J=8.5,5.5 \mathrm{~Hz}, 2 \mathrm{H}), 7.20(\mathrm{t}, J=8.8 \mathrm{~Hz}, 2 \mathrm{H}), 5.52(\mathrm{~s}, 2 \mathrm{H}), 2.52(\mathrm{~s}, 3 \mathrm{H}) ;{ }^{13} \mathrm{C}$ NMR (126 MHz, DMSO) $\delta$ 195.9, $161.9\left(\mathrm{C}-\mathrm{F}, 1 J_{\mathrm{C}-\mathrm{F}}=244.4 \mathrm{~Hz}\right), 153.3,153.0,152.6,132.6$, $132.5,132.2,132.2,130.5,129.5\left(\mathrm{C}-\mathrm{F}, 3 J_{\mathrm{C}-\mathrm{F}}=8.8 \mathrm{~Hz}\right), 124.6,116.1\left(\mathrm{C}-\mathrm{F}, 2 J_{\mathrm{C}-\mathrm{F}}=22.7 \mathrm{~Hz}\right)$, 115.8, 44.5, 25.9; ${ }^{19} \mathrm{~F}$ NMR (471 MHz, DMSO) $\delta$-114.7; HRMS (ESI) m/z: $[\mathrm{M}+\mathrm{Na}]^{+} \mathrm{Calcd}$ for $\mathrm{C}_{18} \mathrm{H}_{14} \mathrm{FN}_{3} \mathrm{O}_{3} \mathrm{Na} 362.0911$; Found 362.0913.

(E)-1-(4-Chlorobenzyl)-3-(1-(hydroxyimino)-2-oxopropyl)quinoxalin-2(1H)-one (18)<smiles>CC(=O)/C(=N/O)c1nc2ccccc2n(Cc2ccc(Cl)cc2)c1=O</smiles>

Purified by recrystallisation using the mixture of ethyl acetate and petroleum ether as solvent. Brown solid (2.20 g, 62\% yield); M.p. $249-250{ }^{\circ} \mathrm{C} .{ }^{1} \mathrm{H}$ NMR (500 MHz, DMSO) $\delta 13.05$ (s, $1 \mathrm{H}), 7.91-7.88(\mathrm{~m}, 1 \mathrm{H}), 7.62(\mathrm{t}, J=7.3 \mathrm{~Hz}, 1 \mathrm{H}), 7.54(\mathrm{~d}, J=8.4 \mathrm{~Hz}, 1 \mathrm{H}), 7.40(\mathrm{dt}, J=5.6$, $2.8 \mathrm{~Hz}, 3 \mathrm{H}), 7.28(\mathrm{~d}, J=8.5 \mathrm{~Hz}, 2 \mathrm{H}), 5.52(\mathrm{~s}, 2 \mathrm{H}), 2.52(\mathrm{~s}, 3 \mathrm{H}) ;{ }^{13} \mathrm{C}$ NMR (126 MHz, DMSO) $\delta 195.9,153.3,152.9,152.5,135.0,132.7,132.7,132.5,132.2,130.5,129.3,129.2,124.7$, 115.7, 44.6, 25.9; HRMS (ESI) m/z: $[\mathrm{M}+\mathrm{Na}]^{+}$Calcd for $\mathrm{C}_{18} \mathrm{H}_{14} \mathrm{ClN}_{3} \mathrm{O}_{3} \mathrm{Na}$ 378.0616; Found 378.0613 .

(E)-3-(1-(Hydroxyimino)-2-oxopropyl)-1,5-dimethylquinoxalin-2(1H)-one (19)<smiles>CC(=O)/C(=N/O)c1nc2c(C)cccc2n(C)c1=O</smiles>

Purified by recrystallisation using the mixture of ethyl acetate and petroleum ether as solvent. Brown solid (1.63 g, 63\% yield); M.p. $225-226{ }^{\circ} \mathrm{C} .{ }^{1} \mathrm{H}$ NMR (500 MHz, DMSO) $\delta 12.88$ (s, $1 \mathrm{H}), 7.62-7.58(\mathrm{~m}, 1 \mathrm{H}), 7.44(\mathrm{~d}, J=8.4 \mathrm{~Hz}, 1 \mathrm{H}), 7.31(\mathrm{~d}, J=7.3 \mathrm{~Hz}, 1 \mathrm{H}), 3.63(\mathrm{~s}, 3 \mathrm{H}), 2.55$ (s, 3H), 2.49 (s, 3H); ${ }^{13} \mathrm{C}$ NMR (126 MHz, DMSO) $\delta$ 195.9, 153.7, 152.6, 150.8, 138.4, 133.8, 
131.9, 130.9, 125.5, 113.5, 29.5, 26.0, 17.6; HRMS (ESI) $\mathrm{m} / \mathrm{z}:[\mathrm{M}+\mathrm{Na}]^{+}$Calcd for $\mathrm{C}_{13} \mathrm{H}_{13} \mathrm{~N}_{3} \mathrm{O}_{3} \mathrm{Na} 282.0849$; Found 282.0850.

(E)-6-Chloro-3-(1-(hydroxyimino)-2-oxopropyl)-1-methylquinoxalin-2(1H)-one (20)<smiles>CC(=O)/C(=N/O)c1nc2cc(Cl)ccc2n(C)c1=O</smiles>

Purified by recrystallisation using the mixture of ethyl acetate and petroleum ether as solvent. Brown solid (1.59 g, 57\% yield); M.p. $231-232{ }^{\circ} \mathrm{C} .{ }^{1} \mathrm{H}$ NMR (500 MHz, DMSO) $\delta 13.13$ (s, $1 \mathrm{H}), 7.96(\mathrm{~d}, J=2.4 \mathrm{~Hz}, 1 \mathrm{H}), 7.78(\mathrm{dd}, J=9.0,2.4 \mathrm{~Hz}, 1 \mathrm{H}), 7.67(\mathrm{~d}, J=9.1 \mathrm{~Hz}, 1 \mathrm{H}), 3.64(\mathrm{~s}$, $3 \mathrm{H}), 2.47$ (s, 3H); ${ }^{13} \mathrm{C}$ NMR (126 MHz, DMSO) $\delta$ 195.8, 154.0, 153.1, 152.5, 132.8, 132.6, 131.8, 129.0, 128.3, 117.6, 29.7, 25.9; HRMS (ESI) m/z: $[\mathrm{M}+\mathrm{Na}]^{+}$Calcd for $\mathrm{C}_{12} \mathrm{H}_{10} \mathrm{ClN}_{3} \mathrm{O}_{3} \mathrm{Na}$ 302.0303; Found 302.0308.

(E)-6-Bromo-3-(1-(hydroxyimino)-2-oxopropyl)-1-methylquinoxalin-2(1H)-one (21)<smiles>CC(=O)/C(=N/O)c1nc2cc(Br)ccc2n(C)c1=O</smiles>

Purified by recrystallisation using the mixture of ethyl acetate and petroleum ether as solvent. Brown solid (1.97 g, 61\% yield); M.p. 238-239 ${ }^{\circ} \mathrm{C} .{ }^{1} \mathrm{H}$ NMR (500 MHz, DMSO) $\delta 12.99$ (s, $1 \mathrm{H}), 7.86(\mathrm{~d}, J=1.9 \mathrm{~Hz}, 1 \mathrm{H}), 7.78(\mathrm{~d}, J=8.5 \mathrm{~Hz}, 1 \mathrm{H}), 7.60(\mathrm{dd}, J=8.5,2.0 \mathrm{~Hz}, 1 \mathrm{H}), 3.63(\mathrm{~s}$, $3 \mathrm{H}), 2.48$ (s, 3H); ${ }^{13} \mathrm{C}$ NMR (126 MHz, DMSO) $\delta$ 195.8, 153.2, 152.8, 152.5, 134.8, 131.7, 131.3, 127.4, 125.4, 118.5, 29.7, 25.9; HRMS (ESI) m/z: $[\mathrm{M}+\mathrm{Na}]^{+}$Calcd for $\mathrm{C}_{12} \mathrm{H}_{10} \mathrm{BrN}_{3} \mathrm{O}_{3} \mathrm{Na}$ 345.9798; Found 345.9802.

(E)-7-Bromo-3-(1-(hydroxyimino)-2-oxopropyl)-1-methylquinoxalin-2(1H)-one (22)<smiles>CC(=O)/C(=N/O)c1nc2ccc(Br)cc2n(C)c1=O</smiles>

Purified by recrystallisation using the mixture of ethyl acetate and petroleum ether as solvent. Brown solid (1.87 g, 58\% yield); M.p. $242-243{ }^{\circ} \mathrm{C} .{ }^{1} \mathrm{H}$ NMR (500 MHz, DMSO) $\delta 13.01$ (s, $1 \mathrm{H}), 8.07(\mathrm{~d}, J=2.3 \mathrm{~Hz}, 1 \mathrm{H}), 7.87(\mathrm{dd}, J=9.0,2.3 \mathrm{~Hz}, 1 \mathrm{H}), 7.59(\mathrm{~d}, J=9.0 \mathrm{~Hz}, 1 \mathrm{H}), 3.63(\mathrm{~s}$, $3 \mathrm{H}), 2.48$ (s, 3H); ${ }^{13} \mathrm{C}$ NMR (126 MHz, DMSO) $\delta 195.8,153.9,153.1,152.5,134.5,133.2$, 133.0, 132.0, 117.9, 116.0, 29.7, 25.9; HRMS (ESI) m/z: $[\mathrm{M}+\mathrm{Na}]^{+}$Calcd for $\mathrm{C}_{12} \mathrm{H}_{10} \mathrm{BrN}_{3} \mathrm{O}_{3} \mathrm{Na}$ 345.9798; Found 345.9800. 
(E)-3-(1-(Hydroxyimino)-2-oxopropyl)-1,6,7-trimethylquinoxalin-2(1H)-one (23)<smiles>CC(=O)/C(=N/O)c1nc2cc(C)c(C)cc2n(C)c1=O</smiles>

Purified by recrystallisation using the mixture of ethyl acetate and petroleum ether as solvent. Brown solid (1.78g, 65\% yield); M.p. 233-234 ${ }^{\circ} \mathrm{C} .{ }^{1} \mathrm{H}$ NMR (500 MHz, DMSO) $\delta 12.85$ (s, $1 \mathrm{H}), 7.59(\mathrm{~s}, 1 \mathrm{H}), 7.41(\mathrm{~s}, 1 \mathrm{H}), 3.60(\mathrm{~s}, 3 \mathrm{H}), 2.47(\mathrm{~s}, 3 \mathrm{H}), 2.40(\mathrm{~s}, 3 \mathrm{H}), 2.30(\mathrm{~s}, 3 \mathrm{H}) ;{ }^{13} \mathrm{C} \mathrm{NMR}$ (126 MHz, DMSO) $\delta 195.9,153.7,152.8,150.8,141.9,133.1,131.6,130.8,129.9,115.7,29.3$, 25.9, 20.5, 19.1; HRMS (ESI) m/z: $[\mathrm{M}+\mathrm{Na}]^{+}$Calcd for $\mathrm{C}_{14} \mathrm{H}_{15} \mathrm{~N}_{3} \mathrm{O}_{3} \mathrm{Na}$ 296.1006; Found 296.1007.

(E)-3-(1-(Hydroxyimino)-2-oxopropyl)-6-(trifluoromethyl)quinoxalin-2(1H)-one (24)<smiles>CC(=O)/C(=N/O)c1nc2cc(C(F)(F)F)ccc2[nH]c1=O</smiles>

Purified by recrystallisation using the mixture of ethyl acetate and petroleum ether as solvent. Brown solid (1.35 g, 45\% yield); M.p. $230-231{ }^{\circ} \mathrm{C} .{ }^{1} \mathrm{H}$ NMR (500 MHz, DMSO) $\delta 13.05$ (s, $1 \mathrm{H}), 13.01(\mathrm{~s}, 1 \mathrm{H}), 8.18(\mathrm{~d}, J=1.1 \mathrm{~Hz}, 1 \mathrm{H}), 7.94(\mathrm{~d}, J=8.7 \mathrm{~Hz}, 1 \mathrm{H}), 7.53(\mathrm{~d}, J=8.6 \mathrm{~Hz}, 1 \mathrm{H})$, $2.48(\mathrm{~s}, 3 \mathrm{H}) ;{ }^{13} \mathrm{C}$ NMR (126 MHz, DMSO) $\delta 195.7,156.0,153.2,152.9,135.3,131.0,128.0$ $\left(\mathrm{C}-\mathrm{F}, 3 J_{\mathrm{C}-\mathrm{F}}=3.8 \mathrm{~Hz}\right), 126.7\left(\mathrm{C}-\mathrm{F}, 3 J_{\mathrm{C}-\mathrm{F}}=3.8 \mathrm{~Hz}\right), 124.6\left(\mathrm{C}-\mathrm{F}, 2 J_{\mathrm{C}-\mathrm{F}}=32.8 \mathrm{~Hz}\right), 124.3(\mathrm{C}-\mathrm{F}$, $1 J_{\mathrm{C}-\mathrm{F}}=272.2 \mathrm{~Hz}$ ), 117.7, 25.9; ${ }^{19} \mathrm{~F}$ NMR (471 MHz, DMSO) $\delta-60.3$; HRMS (ESI) $\mathrm{m} / \mathrm{z}$ : $[\mathrm{M}+\mathrm{Na}]^{+}$Calcd for $\mathrm{C}_{12} \mathrm{H}_{8} \mathrm{~F}_{3} \mathrm{~N}_{3} \mathrm{O}_{3} \mathrm{Na}$ 322.0410; Found 322.0410.

(E)-3-(1-(Hydroxyimino)-2-oxopropyl)-6-nitroquinoxalin-2(1H)-one (25)<smiles>CC(=O)/C(=N/O)c1nc2cc([N+](=O)[O-])ccc2[nH]c1=O</smiles>

Purified by recrystallisation using the mixture of ethyl acetate and petroleum ether as solvent. Brown solid (1.08 g, 39\% yield); M.p. $245-246{ }^{\circ} \mathrm{C} .{ }^{1} \mathrm{H}$ NMR $(500 \mathrm{MHz}, \mathrm{DMSO}) \delta 8.59$ (d, $J=$ $2.5 \mathrm{~Hz}, 1 \mathrm{H}), 8.41(\mathrm{dd}, J=9.1,2.6 \mathrm{~Hz}, 1 \mathrm{H}), 7.52(\mathrm{~d}, J=9.1 \mathrm{~Hz}, 1 \mathrm{H}), 2.48(\mathrm{~s}, 3 \mathrm{H}) ;{ }^{13} \mathrm{C} \mathrm{NMR}$ (126 MHz, DMSO) $\delta 195.8,156.5,154.0,152.9,143.0,138.8,130.7,126.1,125.0,118.1,25.9$; HRMS (ESI) m/z: [M+Na] $]^{+}$Calcd for $\mathrm{C}_{11} \mathrm{H}_{8} \mathrm{~N}_{4} \mathrm{O}_{5} \mathrm{Na} 299.0387$; Found 299.0393.

(E)-3-(1-(Hydroxyimino)-2-oxopropyl)-6,7-dimethylquinoxalin-2(1H)-one (26)<smiles>CC(=O)/C(=N/O)c1nc2cc(C)c(C)cc2[nH]c1=O</smiles> 
Purified by recrystallisation using the mixture of ethyl acetate and petroleum ether as solvent. Brown solid (1.37 g, 53\% yield); M.p. $240-241{ }^{\circ} \mathrm{C} .{ }^{1} \mathrm{H}$ NMR (500 MHz, DMSO) $\delta 12.83$ (s, $1 \mathrm{H}), 12.49(\mathrm{~s}, 1 \mathrm{H}), 7.56(\mathrm{~s}, 1 \mathrm{H}), 7.11(\mathrm{~s}, 1 \mathrm{H}), 2.46(\mathrm{~s}, 3 \mathrm{H}), 2.33(\mathrm{~s}, 3 \mathrm{H}), 2.29(\mathrm{~s}, 3 \mathrm{H}) ;{ }^{13} \mathrm{C} \mathrm{NMR}$ (126 MHz, DMSO) $\delta 195.9,153.6,153.4,152.5,141.5,132.9,130.5,130.4,129.0,116.1,25.9$, 20.3, 19.3; HRMS (ESI) m/z: [M+Na $]^{+}$Calcd for $\mathrm{C}_{13} \mathrm{H}_{13} \mathrm{~N}_{3} \mathrm{O}_{3} \mathrm{Na} 282.0849$; Found 282.0849.

(E)-3-(1-(Hydroxyimino)-2-oxopropyl)quinoxalin-2(1H)-one (27)<smiles>CC(=O)/C(=N/O)c1nc2ccccc2[nH]c1=O</smiles>

Purified by recrystallisation using the mixture of ethyl acetate and petroleum ether as solvent. Brown solid (1.50 g, 65\% yield); M.p. 228-229 ${ }^{\circ} \mathrm{C} .{ }^{1} \mathrm{H}$ NMR (500 MHz, DMSO) $\delta 12.90$ (s, $1 \mathrm{H}), 12.65(\mathrm{~s}, 1 \mathrm{H}), 7.80(\mathrm{dd}, J=8.4,1.3 \mathrm{~Hz}, 1 \mathrm{H}), 7.61(\mathrm{td}, J=7.6,1.3 \mathrm{~Hz}, 1 \mathrm{H}), 7.35$ (dd, $J=$ 11.0, 4.6 Hz, 2H), 2.47 (s, 3H); ${ }^{13} \mathrm{C}$ NMR (126 MHz, DMSO) $\delta$ 195.9, 154.0, 153.4, 153.3, 132.4, 131.9, 131.8, 129.3, 124.1, 116.2, 25.9; HRMS (ESI) $\mathrm{m} / \mathrm{z}:[\mathrm{M}+\mathrm{Na}]^{+}$Calcd for $\mathrm{C}_{11} \mathrm{H}_{9} \mathrm{~N}_{3} \mathrm{O}_{3} \mathrm{Na} 254.0536$; Found 254.0537.

(E)-3-(1-(Hydroxyimino)-2-oxobutyl)-1-methylquinoxalin-2(1H)-one (28)<smiles>CCC(=O)/C(=N/O)c1nc2ccccc2n(C)c1=O</smiles>

Purified by recrystallisation using the mixture of ethyl acetate and petroleum ether as solvent. Brown solid (1.97 g, 76\% yield); M.p. $227-228{ }^{\circ} \mathrm{C} .{ }^{1} \mathrm{H}$ NMR (500 MHz, DMSO) $\delta 12.98$ (s, $1 \mathrm{H}), 7.86(\mathrm{dd}, J=8.0,1.4 \mathrm{~Hz}, 1 \mathrm{H}), 7.73(\mathrm{ddd}, J=8.6,7.2,1.5 \mathrm{~Hz}, 1 \mathrm{H}), 7.64(\mathrm{dd}, J=8.5,1.0$ $\mathrm{Hz}, 1 \mathrm{H}), 7.47-7.43(\mathrm{~m}, 1 \mathrm{H}), 3.65(\mathrm{~s}, 3 \mathrm{H}), 2.95(\mathrm{q}, J=7.3 \mathrm{~Hz}, 2 \mathrm{H}), 1.07(\mathrm{t}, J=7.3 \mathrm{~Hz}, 3 \mathrm{H})$; ${ }^{13} \mathrm{C}$ NMR (126 MHz, DMSO) $\delta$ 198.5, 152.8, 152.8, 152.5, 133.6, 132.3, 132.1, 130.1, 124.4, 115.6, 31.0, 29.4, 8.3; HRMS (ESI) m/z: [M+Na $]^{+}$Calcd for $\mathrm{C}_{13} \mathrm{H}_{13} \mathrm{~N}_{3} \mathrm{O}_{3} \mathrm{Na}$ 282.0849; Found 282.0858 .

(E)-3-(1-(Hydroxyimino)-3-methyl-2-oxobutyl)-1-methylquinoxalin-2(1H)-one (29)<smiles>CC(C)C(=O)/C(=N/O)c1nc2ccccc2n(C)c1=O</smiles>

Purified by recrystallisation using the mixture of ethyl acetate and petroleum ether as solvent. Brown solid (2.13 g, 78\% yield); M.p. 220-221 ${ }^{\circ} \mathrm{C} .{ }^{1} \mathrm{H}$ NMR (500 MHz, DMSO) $\delta 12.89$ (s, $1 \mathrm{H}), 7.87(\mathrm{~d}, J=7.9 \mathrm{~Hz}, 1 \mathrm{H}), 7.74-7.70(\mathrm{~m}, 1 \mathrm{H}), 7.62(\mathrm{~d}, J=8.3 \mathrm{~Hz}, 1 \mathrm{H}), 7.44(\mathrm{t}, J=7.5 \mathrm{~Hz}$, $1 \mathrm{H}), 3.65(\mathrm{~s}, 3 \mathrm{H}), 3.62(\mathrm{dd}, J=11.4,4.4 \mathrm{~Hz}, 1 \mathrm{H}), 1.15(\mathrm{~d}, J=6.9 \mathrm{~Hz}, 6 \mathrm{H}) ;{ }^{13} \mathrm{C} \mathrm{NMR}(126$ 
MHz, DMSO) $\delta$ 201.6, 152.7, 152.6, 151.9, 133.6, 132.3, 132.0, 130.1, 124.3, 115.6, 35.1, 29.4, 19.0; HRMS (ESI) m/z: [M+Na $]^{+}$Calcd for $\mathrm{C}_{14} \mathrm{H}_{15} \mathrm{~N}_{3} \mathrm{O}_{3} \mathrm{Na} 296.1006$; Found 296.1005.

(E)-3-(1-(Hydroxyimino)-2-oxopentyl)-1-methylquinoxalin-2(1H)-one (30)<smiles>CCCC(=O)/C(=N/O)c1nc2ccccc2n(C)c1=O</smiles>

Purified by recrystallisation using the mixture of ethyl acetate and petroleum ether as solvent. Brown solid (1.99 g, 73\% yield); M.p. $237-238{ }^{\circ} \mathrm{C} .{ }^{1} \mathrm{H}$ NMR (500 MHz, DMSO) $\delta 12.88$ (s, $1 \mathrm{H}), 7.86(\mathrm{dd}, J=8.0,1.3 \mathrm{~Hz}, 1 \mathrm{H}), 7.73(\mathrm{ddd}, J=8.6,7.3,1.5 \mathrm{~Hz}, 1 \mathrm{H}), 7.63(\mathrm{dd}, J=8.5,0.9$ $\mathrm{Hz}, 1 \mathrm{H}), 7.47-7.41(\mathrm{~m}, 1 \mathrm{H}), 3.65(\mathrm{~s}, 3 \mathrm{H}), 2.89(\mathrm{t}, J=7.2 \mathrm{~Hz}, 2 \mathrm{H}), 1.63(\mathrm{dd}, J=14.7,7.3 \mathrm{~Hz}$, 2H), $0.94(\mathrm{t}, J=7.6 \mathrm{~Hz}, 3 \mathrm{H}) ;{ }^{13} \mathrm{C}$ NMR (126 MHz, DMSO) $\delta 198.1,153.1,152.8,152.5,133.6$, 132.3, 132.0, 130.1, 124.3, 115.6, 29.4, 17.7, 14.1; HRMS (ESI) m/z: [M+Na] ${ }^{+}$Calcd for $\mathrm{C}_{14} \mathrm{H}_{15} \mathrm{~N}_{3} \mathrm{O}_{3} \mathrm{Na} 296.1006$; Found 296.1006.

(E)-3-(1-(hydroxyimino)-5-methyl-2-oxohexyl)-1-methylquinoxalin-2(1H)-one (31)<smiles>CC(C)CCC(=O)/C(=N/O)c1nc2ccccc2n(C)c1=O</smiles>

Purified by recrystallisation using the mixture of ethyl acetate and petroleum ether as solvent. Brown solid (1.87 g, 62\% yield); M.p. $242-243{ }^{\circ} \mathrm{C} .{ }^{1} \mathrm{H}$ NMR (500 MHz, DMSO) $\delta 12.87$ (s, $1 \mathrm{H}), 7.87(\mathrm{dd}, J=8.0,1.3 \mathrm{~Hz}, 1 \mathrm{H}), 7.76-7.72(\mathrm{~m}, 1 \mathrm{H}), 7.64(\mathrm{~d}, J=7.9 \mathrm{~Hz}, 1 \mathrm{H}), 7.47-7.43$ $(\mathrm{m}, 1 \mathrm{H}), 3.65(\mathrm{~s}, 3 \mathrm{H}), 2.94-2.89(\mathrm{~m}, 2 \mathrm{H}), 1.60(\mathrm{dt}, J=13.3,6.6 \mathrm{~Hz}, 1 \mathrm{H}), 1.51(\mathrm{dd}, J=14.9$, $7.2 \mathrm{~Hz}, 2 \mathrm{H}), 0.91$ (d, $J=6.6 \mathrm{~Hz}, 6 \mathrm{H}) ;{ }^{13} \mathrm{C} \mathrm{NMR}$ (126 MHz, DMSO) $\delta 198.3,153.0,152.7$, 152.5, 133.6, 132.3, 132.1, 130.1, 124.4, 115.7, 35.6, 33.1, 29.4, 27.7, 22.8; HRMS (ESI) m/z: $[\mathrm{M}+\mathrm{Na}]^{+}$Calcd for $\mathrm{C}_{16} \mathrm{H}_{19} \mathrm{~N}_{3} \mathrm{O}_{3} \mathrm{Na}$ 324.1319; Found 324.1319.

\section{(E)-3-(1-(Hydroxyimino)-2-oxohexyl)-1-methylquinoxalin-2(1H)-one (32)}<smiles>CCCCC(=O)/C(=N/O)c1nc2ccccc2n(C)c1=O</smiles>

Purified by recrystallisation using the mixture of ethyl acetate and petroleum ether as solvent. Brown solid (2.01 g, 70\% yield); M.p. 239-240 ${ }^{\circ} \mathrm{C} .{ }^{1} \mathrm{H}$ NMR (500 MHz, DMSO) $\delta 12.86$ (s, $1 \mathrm{H}), 7.87(\mathrm{dd}, J=8.0,1.2 \mathrm{~Hz}, 1 \mathrm{H}), 7.75-7.69(\mathrm{~m}, 1 \mathrm{H}), 7.63(\mathrm{~d}, J=8.1 \mathrm{~Hz}, 1 \mathrm{H}), 7.47-7.41$ $(\mathrm{m}, 1 \mathrm{H}), 3.65(\mathrm{~s}, 3 \mathrm{H}), 2.92(\mathrm{t}, J=7.4 \mathrm{~Hz}, 2 \mathrm{H}), 1.63-1.56(\mathrm{~m}, 2 \mathrm{H}), 1.40-1.32(\mathrm{~m}, 2 \mathrm{H}), 0.91(\mathrm{t}$, $J=7.4 \mathrm{~Hz}, 3 \mathrm{H}) ;{ }^{13} \mathrm{C} \mathrm{NMR}(101 \mathrm{MHz}, \mathrm{DMSO}) \delta 197.7,152.5,152.2,152.0,133.1,131.8$, 131.5, 129.6, 123.9, 115.1, 36.8, 28.9, 25.9, 21.7, 13.7; HRMS (ESI) m/z: [M+Na] ${ }^{+}$Calcd for $\mathrm{C}_{15} \mathrm{H}_{17} \mathrm{~N}_{3} \mathrm{O}_{3} \mathrm{Na}$ 310.1162; Found 310.1162. 
(E)-3-(1-(Hydroxyimino)-2-oxoheptyl)-1-methylquinoxalin-2(1H)-one (33)<smiles>CCCCCC(=O)/C(=N/O)c1nc2ccccc2n(C)c1=O</smiles>

Purified by recrystallisation using the mixture of ethyl acetate and petroleum ether as solvent. Brown solid (1.96 g, 65\% yield); M.p. $245-246{ }^{\circ} \mathrm{C} .{ }^{1} \mathrm{H}$ NMR (500 MHz, DMSO) $\delta 12.88$ (s, $1 \mathrm{H}), 7.86(\mathrm{~d}, J=7.8 \mathrm{~Hz}, 1 \mathrm{H}), 7.70(\mathrm{t}, J=7.6 \mathrm{~Hz}, 1 \mathrm{H}), 7.60$ (d, $J=8.4 \mathrm{~Hz}, 1 \mathrm{H}), 7.43$ (t, $J=7.5$ $\mathrm{Hz}, 1 \mathrm{H}), 3.64(\mathrm{~s}, 3 \mathrm{H}), 2.92(\mathrm{t}, J=7.3 \mathrm{~Hz}, 2 \mathrm{H}), 1.67-1.58(\mathrm{~m}, 2 \mathrm{H}), 1.31(\mathrm{~d}, J=3.5 \mathrm{~Hz}, 4 \mathrm{H})$, $0.89(\mathrm{~d}, J=6.6 \mathrm{~Hz}, 3 \mathrm{H}) ;{ }^{13} \mathrm{C}$ NMR $(126 \mathrm{MHz}, \mathrm{DMSO}) \delta 198.2,153.1,152.8,152.5,133.5$, 132.3, 132.0, 130.1, 124.3, 115.5, 37.5, 31.3, 29.4, 23.9, 22.4, 14.3; HRMS (ESI) m/z: $[\mathrm{M}+\mathrm{Na}]^{+}$Calcd for $\mathrm{C}_{16} \mathrm{H}_{19} \mathrm{~N}_{3} \mathrm{O}_{3} \mathrm{Na}$ 324.1319; Found 324.1318.

(E)-3-(1-(hydroxyimino)-2-oxooctyl)-1-methylquinoxalin-2(1H)-one (34)<smiles>CCCCCCC(=O)/C(=N/O)c1nc2ccccc2n(C)c1=O</smiles>

Purified by recrystallisation using the mixture of ethyl acetate and petroleum ether as solvent. Brown solid (2.08 g, 66\% yield); M.p. 250-251 ${ }^{\circ} \mathrm{C} .{ }^{1} \mathrm{H}$ NMR (500 MHz, DMSO) $\delta 12.87$ (s, $1 \mathrm{H}), 7.86(\mathrm{dd}, J=8.0,1.2 \mathrm{~Hz}, 1 \mathrm{H}), 7.74-7.69(\mathrm{~m}, 1 \mathrm{H}), 7.62(\mathrm{~d}, J=8.1 \mathrm{~Hz}, 1 \mathrm{H}), 7.46-7.41$ $(\mathrm{m}, 1 \mathrm{H}), 3.65(\mathrm{~s}, 3 \mathrm{H}), 2.92(\mathrm{t}, J=7.4 \mathrm{~Hz}, 2 \mathrm{H}), 1.64-1.57(\mathrm{~m}, 2 \mathrm{H}), 1.31(\mathrm{dt}, J=10.3,6.9 \mathrm{~Hz}$, $6 \mathrm{H}), 0.87(\mathrm{t}, J=6.9 \mathrm{~Hz}, 3 \mathrm{H}) ;{ }^{13} \mathrm{C}$ NMR (101 MHz, DMSO) $\delta 197.6,152.5,152.2,152.0,133.1$, 131.8, 131.5, 129.6, 123.8, 115.1, 37.1, 31.0, 28.9, 28.2, 23.7, 21.9, 13.9; HRMS (ESI) m/z: $[\mathrm{M}+\mathrm{Na}]^{+}$Calcd for $\mathrm{C}_{17} \mathrm{H}_{21} \mathrm{~N}_{3} \mathrm{O}_{3} \mathrm{Na} 338.1475$; Found 338.1473.

(E)-3-(1-(hydroxyimino)-2-oxononyl)-1-methylquinoxalin-2(1H)-one (35)<smiles>CCCCCCCC(=O)/C(=N/O)c1nc2ccccc2n(C)c1=O</smiles>

Purified by recrystallisation using the mixture of ethyl acetate and petroleum ether as solvent. Brown solid (2.34 g, 71\% yield); M.p. 256-257 ${ }^{\circ} \mathrm{C} .{ }^{1} \mathrm{H}$ NMR (500 MHz, DMSO) $\delta 12.86$ (s, $1 \mathrm{H}), 7.86(\mathrm{dd}, J=8.0,1.2 \mathrm{~Hz}, 1 \mathrm{H}), 7.74-7.69(\mathrm{~m}, 1 \mathrm{H}), 7.62(\mathrm{~d}, J=8.1 \mathrm{~Hz}, 1 \mathrm{H}), 7.46-7.41$ $(\mathrm{m}, 1 \mathrm{H}), 3.65(\mathrm{~s}, 3 \mathrm{H}), 2.91(\mathrm{t}, J=7.4 \mathrm{~Hz}, 2 \mathrm{H}), 1.60(\mathrm{dd}, J=14.4,7.2 \mathrm{~Hz}, 2 \mathrm{H}), 1.34-1.25(\mathrm{~m}$, $8 \mathrm{H}), 0.87(\mathrm{t}, J=6.9 \mathrm{~Hz}, 3 \mathrm{H}) ;{ }^{13} \mathrm{C}$ NMR (101 MHz, DMSO) $\delta 197.6,152.5,152.2,152.0,133.1$, 131.8, 131.5, 129.6, 123.8, 115.1, 37.1, 31.1, 28.9, 28.5, 28.5, 23.7, 22.0, 13.9; HRMS (ESI) $\mathrm{m} / \mathrm{z}:[\mathrm{M}+\mathrm{Na}]^{+}$Calcd for $\mathrm{C}_{18} \mathrm{H}_{23} \mathrm{~N}_{3} \mathrm{O}_{3} \mathrm{Na} 352.1632$; Found 352.1632. 
(E)-3-(1-(hydroxyimino)-2-oxoundecyl)-1-methylquinoxalin-2(1H)-one (36)<smiles>CCCCCCCCCC(=O)/C(=N/O)c1nc2ccccc2n(C)c1=O</smiles>

Purified by recrystallisation using the mixture of ethyl acetate and petroleum ether as solvent. Brown solid (1.96 g, 55\% yield); M.p. 259-260 ${ }^{\circ} \mathrm{C} .{ }^{1} \mathrm{H}$ NMR (500 MHz, DMSO) $\delta 12.97$ (s, $1 \mathrm{H}), 7.86(\mathrm{dd}, J=8.0,1.2 \mathrm{~Hz}, 1 \mathrm{H}), 7.75-7.70(\mathrm{~m}, 1 \mathrm{H}), 7.63(\mathrm{~d}, J=8.4 \mathrm{~Hz}, 1 \mathrm{H}), 7.47-7.41$ $(\mathrm{m}, 1 \mathrm{H}), 3.65(\mathrm{~s}, 3 \mathrm{H}), 2.90(\mathrm{t}, J=7.3 \mathrm{~Hz}, 2 \mathrm{H}), 1.59(\mathrm{dd}, J=14.4,7.2 \mathrm{~Hz}, 2 \mathrm{H}), 1.33-1.23(\mathrm{~m}$, $12 \mathrm{H}), 0.87(\mathrm{t}, J=6.8 \mathrm{~Hz}, 3 \mathrm{H}) ;{ }^{13} \mathrm{C}$ NMR $(101 \mathrm{MHz}, \mathrm{DMSO}) \delta 197.7,152.5,152.3,152.2$, $133.1,131.8,131.5,129.6,123.8,115.1,37.0,31.3,28.9,28.6,28.6,28.6,23.7,22.1$, 13.9; HRMS (ESI) m/z: [M+Na] $]^{+}$Calcd for $\mathrm{C}_{20} \mathrm{H}_{27} \mathrm{~N}_{3} \mathrm{O}_{3} \mathrm{Na}$ 380.1945; Found 380.1945.

(E)-3-(2-Cyclopropyl-1-(hydroxyimino)-2-oxoethyl)-1-methylquinoxalin-2(1H)-one (37)<smiles>Cn1c(=O)c(/C(=N\O)C(=O)C2CC2)nc2ccccc21</smiles>

Purified by recrystallisation using the mixture of ethyl acetate and petroleum ether as solvent. Brown solid (1.95 g, 72\% yield); M.p. 208-209 ${ }^{\circ} \mathrm{C} .{ }^{1} \mathrm{H}$ NMR (500 MHz, DMSO) $\delta 12.99$ (s, $1 \mathrm{H}), 7.87(\mathrm{dd}, J=8.0,1.3 \mathrm{~Hz}, 1 \mathrm{H}), 7.74-7.68(\mathrm{~m}, 1 \mathrm{H}), 7.61(\mathrm{~d}, J=7.8 \mathrm{~Hz}, 1 \mathrm{H}), 7.46-7.40$ $(\mathrm{m}, 1 \mathrm{H}), 3.64(\mathrm{~s}, 3 \mathrm{H}), 2.99-2.92(\mathrm{~m}, 1 \mathrm{H}), 1.09(\mathrm{dd}, J=7.3,4.1 \mathrm{~Hz}, 2 \mathrm{H}), 1.01(\mathrm{dt}, J=7.2,3.4$ $\mathrm{Hz}, 2 \mathrm{H}) ;{ }^{13} \mathrm{C}$ NMR (126 MHz, DMSO) $\delta$ 197.2, 153.5, 152.7, 152.5, 133.6, 132.3, 132.1, 130.1, 124.4, 115.6, 29.4, 16.4, 11.8; HRMS (ESI) m/z: [M+Na $]^{+}$Calcd for $\mathrm{C}_{14} \mathrm{H}_{13} \mathrm{~N}_{3} \mathrm{O}_{3} \mathrm{Na}$ 294.0849; Found 294.0849.

(E)-3-(2-cyclopentyl-1-(hydroxyimino)-2-oxoethyl)-1-methylquinoxalin-2(1H)-one (38)<smiles>Cn1c(=O)c(/C(=N\O)C(=O)C2CCCC2)nc2ccccc21</smiles>

Purified by recrystallisation using the mixture of ethyl acetate and petroleum ether as solvent. Brown solid (2.06 g, 69\% yield); M.p. $219-220{ }^{\circ} \mathrm{C} .{ }^{1} \mathrm{H}$ NMR (500 MHz, DMSO) $\delta 12.85$ (s, $1 \mathrm{H}), 7.89-7.85(\mathrm{~m}, 1 \mathrm{H}), 7.73(\mathrm{t}, J=7.8 \mathrm{~Hz}, 1 \mathrm{H}), 7.64(\mathrm{~d}, J=8.4 \mathrm{~Hz}, 1 \mathrm{H}), 7.45(\mathrm{t}, J=7.6 \mathrm{~Hz}$, $1 \mathrm{H}), 3.83-3.76(\mathrm{~m}, 1 \mathrm{H}), 3.65(\mathrm{~s}, 3 \mathrm{H}), 1.92-1.86(\mathrm{~m}, 2 \mathrm{H}), 1.83-1.76(\mathrm{~m}, 2 \mathrm{H}), 1.64-1.58$ (m, 4H) ${ }^{13} \mathrm{C}$ NMR (101 MHz, DMSO) $\delta$ 199.6, 152.3, 152.2, 152.2, 133.1, 131.8, 131.5, 129.6, 123.9, 115.1, 45.1, 29.1, 28.9, 25.8; HRMS (ESI) m/z: [M+Na] $]^{+}$Calcd for $\mathrm{C}_{16} \mathrm{H}_{17} \mathrm{~N}_{3} \mathrm{O}_{3} \mathrm{Na}$ 322.1162; Found 322.1164. 
(E)-3-(2-Cyclohexyl-1-(hydroxyimino)-2-oxoethyl)-1-methylquinoxalin-2(1H)-one (39)<smiles>Cn1c(=O)c(/C(=N\O)C(=O)C2CCCCC2)nc2ccccc21</smiles>

Purified by recrystallisation using the mixture of ethyl acetate and petroleum ether as solvent. Brown solid (2.35 g, 75\% yield); M.p. $231-232{ }^{\circ} \mathrm{C} .{ }^{1} \mathrm{H}$ NMR (500 MHz, DMSO) $\delta 12.86$ (s, $1 \mathrm{H}), 7.87(\mathrm{dd}, J=8.0,1.3 \mathrm{~Hz}, 1 \mathrm{H}), 7.75-7.71(\mathrm{~m}, 1 \mathrm{H}), 7.64(\mathrm{~d}, J=7.8 \mathrm{~Hz}, 1 \mathrm{H}), 7.47-7.43$ $(\mathrm{m}, 1 \mathrm{H}), 3.65(\mathrm{~s}, 3 \mathrm{H}), 3.38(\mathrm{dd}, J=7.0,3.8 \mathrm{~Hz}, 1 \mathrm{H}), 1.89(\mathrm{~d}, J=8.2 \mathrm{~Hz}, 2 \mathrm{H}), 1.82-1.76(\mathrm{~m}$, $2 \mathrm{H}), 1.68(\mathrm{~d}, J=12.1 \mathrm{~Hz}, 1 \mathrm{H}), 1.40-1.32(\mathrm{~m}, 4 \mathrm{H}), 1.20(\mathrm{dd}, J=9.6,7.4 \mathrm{~Hz}, 1 \mathrm{H}) ;{ }^{13} \mathrm{C} \mathrm{NMR}$ (101 MHz, DMSO) $\delta$ 200.2, 152.2, 152.0, 151.4, 133.1, 131.8, 131.5, 129.6, 123.9, 115.1, 44.5, 28.9, 28.5, 25.4, 25.2; HRMS (ESI) $\mathrm{m} / \mathrm{z}$ : $[\mathrm{M}+\mathrm{Na}]^{+}$Calcd for $\mathrm{C}_{17} \mathrm{H}_{19} \mathrm{~N}_{3} \mathrm{O}_{3} \mathrm{Na}$ 336.1319; Found 336.1323.

(E)-3-(1-(hydroxyimino)-4-(4-methoxyphenyl)-2-oxobutyl)-1-methylquinoxalin-2(1H)-one (40)<smiles>COc1ccc(CCC(=O)/C(=N/O)c2nc3ccccc3n(C)c2=O)cc1</smiles>

Purified by recrystallisation using the mixture of ethyl acetate and petroleum ether as solvent. Brown solid (1.15 g, 63\% yield); M.p. 286-287 ${ }^{\circ} \mathrm{C} .{ }^{1} \mathrm{H}$ NMR (500 MHz, DMSO) $\delta 12.98$ (s, $1 \mathrm{H}), 7.86(\mathrm{~d}, J=8.0 \mathrm{~Hz}, 1 \mathrm{H}), 7.75-7.71(\mathrm{~m}, 1 \mathrm{H}), 7.63(\mathrm{~d}, J=8.4 \mathrm{~Hz}, 1 \mathrm{H}), 7.44(\mathrm{t}, J=7.6 \mathrm{~Hz}$, $1 \mathrm{H}), 7.19$ (d, $J=8.5 \mathrm{~Hz}, 2 \mathrm{H}), 6.86(\mathrm{~d}, J=8.5 \mathrm{~Hz}, 2 \mathrm{H}), 3.73(\mathrm{~s}, 3 \mathrm{H}), 3.65(\mathrm{~s}, 3 \mathrm{H}), 3.22(\mathrm{t}, J=$ $7.6 \mathrm{~Hz}, 2 \mathrm{H}), 2.86(\mathrm{t}, J=7.6 \mathrm{~Hz}, 2 \mathrm{H}) ;{ }^{13} \mathrm{C} \mathrm{NMR}(126 \mathrm{MHz}, \mathrm{DMSO}) \delta 197.2,158.0,152.9$, 152.8, 152.5, 133.6, 133.3, 132.3, 132.1, 130.1, 129.7, 124.4, 115.6, 114.2, 55.4, 39.6, 29.4, 28.8; HRMS (ESI) m/z: [M+Na] $]^{+}$Calcd for $\mathrm{C}_{20} \mathrm{H}_{19} \mathrm{~N}_{3} \mathrm{O}_{4} \mathrm{Na} 388.1268$; Found 388.1266.

(E)-3-(4-(4-hydroxy-3-methoxyphenyl)-1-(hydroxyimino)-2-oxobutyl)-1-methylquinoxalin -2(1H)-one (41)<smiles>COc1cc(CCC(=O)/C(=N/O)c2nc3ccccc3n(C)c2=O)ccc1O</smiles>

Purified by recrystallisation using the mixture of ethyl acetate and petroleum ether as solvent. Brown solid (1.01 g, 53\% yield); M.p. 311-312 ${ }^{\circ} \mathrm{C} .{ }^{1} \mathrm{H}$ NMR (500 MHz, DMSO) $\delta 12.94$ (s, $1 \mathrm{H}), 10.19(\mathrm{~s}, 1 \mathrm{H}), 7.87-7.84(\mathrm{~m}, 1 \mathrm{H}), 7.75-7.71(\mathrm{~m}, 1 \mathrm{H}), 7.64(\mathrm{~d}, J=8.2 \mathrm{~Hz}, 1 \mathrm{H}), 7.54-$ $7.30(\mathrm{~m}, 3 \mathrm{H}), 7.25(\mathrm{~d}, J=1.7 \mathrm{~Hz}, 1 \mathrm{H}), 3.89(\mathrm{~s}, 3 \mathrm{H}), 3.65(\mathrm{~s}, 3 \mathrm{H}), 3.29(\mathrm{t}, J=7.5 \mathrm{~Hz}, 2 \mathrm{H}), 2.91$ $(\mathrm{t}, J=7.5 \mathrm{~Hz}, 2 \mathrm{H}) ;{ }^{13} \mathrm{C}$ NMR (126 MHz, DMSO) $\delta 197.1,152.9,152.7,152.4,149.7,141.3$, 137.2, 133.6, 132.3, 132.1, 132.1, 130.1, 124.4, 117.4, 115.6, 115.5, 57.0, 39.1, 29.4, 29.0; HRMS (ESI) m/z: [M+Na] $]^{+}$Calcd for $\mathrm{C}_{20} \mathrm{H}_{19} \mathrm{~N}_{3} \mathrm{O}_{5} \mathrm{Na}$ 404.1217; Found 404.1231. 
(E)-3-(1-(hydroxyimino)-4-(6-methoxynaphthalen-2-yl)-2-oxobutyl)-1-methylquinoxalin-2 (1H)-one (42)<smiles>COc1ccc2cc(CCC(=O)/C(=N/O)c3nc4ccccc4n(C)c3=O)ccc2c1</smiles>

Purified by recrystallisation using the mixture of ethyl acetate and petroleum ether as solvent. Brown solid (1.04 g, 50\% yield); M.p. 317-318 ${ }^{\circ} \mathrm{C} .{ }^{1} \mathrm{H}$ NMR $(500 \mathrm{MHz}, \mathrm{DMSO}) \delta 7.85$ (d, $J=$ $7.9 \mathrm{~Hz}, 1 \mathrm{H}), 7.79-7.70(\mathrm{~m}, 3 \mathrm{H}), 7.68(\mathrm{~s}, 1 \mathrm{H}), 7.63(\mathrm{~d}, J=8.4 \mathrm{~Hz}, 1 \mathrm{H}), 7.48-7.38(\mathrm{~m}, 2 \mathrm{H})$, $7.28(\mathrm{~d}, J=2.1 \mathrm{~Hz}, 1 \mathrm{H}), 7.13(\mathrm{dd}, J=8.9,2.4 \mathrm{~Hz}, 1 \mathrm{H}), 3.86(\mathrm{~s}, 3 \mathrm{H}), 3.65(\mathrm{~s}, 3 \mathrm{H}), 3.32(\mathrm{t}, J=$ $7.5 \mathrm{~Hz}, 2 \mathrm{H}), 3.04(\mathrm{t}, J=7.5 \mathrm{~Hz}, 2 \mathrm{H}) ;{ }^{13} \mathrm{C} \mathrm{NMR}(126 \mathrm{MHz}, \mathrm{DMSO}) \delta 197.2,157.3,153.0$, $152.8,136.6,133.6,133.3,132.4,132.0,130.1,129.3,129.1,128.2,127.2$, 126.5, 124.3, 119.0, 115.6, 106.2, 100.0, 55.6, 39.2, 29.8, 29.4; HRMS (ESI) m/z: $[\mathrm{M}+\mathrm{Na}]^{+}$Calcd for $\mathrm{C}_{24} \mathrm{H}_{21} \mathrm{~N}_{3} \mathrm{O}_{4} \mathrm{Na} 438.1424$; Found 438.1422.

3-((E)-2-((5S,8R,9S,10S,13S,14S,17S)-10,13-dimethyl-3-oxohexadecahydro-1H-cyclopenta [a]phenanthren-17-yl)-1-(hydroxyimino)-2-oxoethyl)-1-methylquinoxalin-2(1H)-one (43)

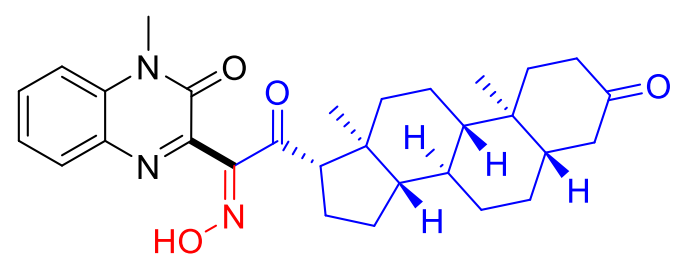

Purified by recrystallisation using the mixture of ethyl acetate and petroleum ether as solvent. Brown solid (1.00 g, 40\% yield); M.p. $399-400{ }^{\circ} \mathrm{C} .{ }^{1} \mathrm{H}$ NMR (500 MHz, DMSO) $\delta 7.85$ (dd, $J$ $=8.0,1.3 \mathrm{~Hz}, 1 \mathrm{H}), 7.69-7.64(\mathrm{~m}, 1 \mathrm{H}), 7.58(\mathrm{~d}, J=7.7 \mathrm{~Hz}, 1 \mathrm{H}), 7.42-7.38(\mathrm{~m}, 1 \mathrm{H}), 3.61(\mathrm{~s}$, $3 \mathrm{H}), 2.61-2.59(\mathrm{~m} \mathrm{1H}), 2.31-2.27(\mathrm{~m}, 1 \mathrm{H}), 2.07-2.06(\mathrm{~m}, 5 \mathrm{H}), 2.04-1.95(\mathrm{~m}, 4 \mathrm{H}), 1.88-1.84$ (m, 1H), 1.69-1.64 (m, 4H), 1.48-1.45 (m, 6H), 1.09 (s, 3H), $0.86(\mathrm{t}, J=6.9 \mathrm{~Hz}, 1 \mathrm{H}), 0.58$ (s, $3 \mathrm{H}) ;{ }^{13} \mathrm{C}$ NMR (126 MHz, DMSO) $\delta 213.7,201.5,161.7,161.4,158.6,141.5,138.7,137.3$, 134.9, 128.7, 120.0, 67.8, 61.0, 54.5, 49.0, 48.8, 45.6, 44.1, 43.2, 40.3, 36.4, 36.0, 34.1, 32.0, 30.3, 29.1, 27.5, 26.0, 18.5, 17.9; HRMS (ESI) m/z: $[\mathrm{M}+\mathrm{Na}]^{+}$Calcd for $\mathrm{C}_{30} \mathrm{H}_{37} \mathrm{~N}_{3} \mathrm{O}_{4} \mathrm{Na}$ 526.2676; Found 526.2668.

\section{4-Methyl-3-oxo-3,4-dihydroquinoxaline-2-carbonitrile (44) ${ }^{2}$}<smiles>Cn1c(=O)c(C#N)nc2ccccc21</smiles>

Purified by silica gel column chromatography using the mixture of ethyl acetate and petroleum ether as eluant. White solid (311 mg, 84\% yield); ${ }^{1} \mathrm{H}$ NMR (500 MHz, DMSO) $\delta 7.93$ (d, $J=$ $8.0 \mathrm{~Hz}, 1 \mathrm{H}), 7.86(\mathrm{dd}, J=11.5,4.2 \mathrm{~Hz}, 1 \mathrm{H}), 7.69(\mathrm{~d}, J=8.5 \mathrm{~Hz}, 1 \mathrm{H}), 7.51(\mathrm{t}, J=7.6 \mathrm{~Hz}, 1 \mathrm{H})$, $3.66(\mathrm{~s}, 3 \mathrm{H}) ;{ }^{13} \mathrm{C}$ NMR (126 MHz, DMSO) $\delta 153.4,135.0,134.8,134.1,132.8,131.1,125.0$, $116.0,115.4,30.1$. 
1-Methyl-3-nitroquinoxalin-2(1H)-one (45)<smiles>Cn1c(=O)c([N+](=O)[O-])nc2ccccc21</smiles>

Purified by silica gel column chromatography using the mixture of ethyl acetate and petroleum ether as eluant. Yellow solid (373 mg, 91\% yield); M.p. $171-172{ }^{\circ} \mathrm{C} .{ }^{1} \mathrm{H}$ NMR $(500 \mathrm{MHz}$, $\left.\mathrm{CDCl}_{3}\right) \delta 7.98(\mathrm{dd}, J=8.1,1.2 \mathrm{~Hz}, 1 \mathrm{H}), 7.89-7.83(\mathrm{~m}, 1 \mathrm{H}), 7.53(\mathrm{dd}, J=17.3,8.1 \mathrm{~Hz}, 2 \mathrm{H})$, $3.80(\mathrm{~s}, 3 \mathrm{H}) ;{ }^{13} \mathrm{C}$ NMR $\left(126 \mathrm{MHz}, \mathrm{CDCl}_{3}\right) \delta 152.4,141.2,135.2,134.8,132.3,131.5,125.4$, 114.5, 29.8; HRMS (ESI) m/z: [M+Na] ${ }^{+}$Calcd for $\mathrm{C}_{9} \mathrm{H}_{7} \mathrm{~N}_{3} \mathrm{O}_{3} \mathrm{Na}$ 228.0380; Found 228.0373.

(E)-3-(1-(Methoxyimino)-2-oxopropyl)-1-methylquinoxalin-2(1H)-one (46)<smiles>CO/N=C(/C(C)=O)c1nc2ccccc2n(C)c1=O</smiles>

Purified by silica gel column chromatography using the mixture of ethyl acetate and petroleum ether as eluant. Yellow solid (487 mg, 94\% yield); M.p. 199-200 ${ }^{\circ} \mathrm{C} .{ }^{1} \mathrm{H}$ NMR $(500 \mathrm{MHz}$, $\left.\mathrm{CDCl}_{3}\right) \delta 7.91(\mathrm{~d}, J=7.4 \mathrm{~Hz}, 1 \mathrm{H}), 7.65-7.59(\mathrm{~m}, 1 \mathrm{H}), 7.37(\mathrm{dd}, J=16.9,8.4 \mathrm{~Hz}, 2 \mathrm{H}), 4.09(\mathrm{~s}$, $3 \mathrm{H}), 3.70(\mathrm{~s}, 3 \mathrm{H}), 2.56(\mathrm{~s}, 3 \mathrm{H}) ;{ }^{13} \mathrm{C} \mathrm{NMR}\left(126 \mathrm{MHz}, \mathrm{CDCl}_{3}\right) \delta 195.0,152.9,152.4,151.3$, 133.5, 132.8, 131.5, 130.8, 123.9, 113.9, 64.3, 29.1, 25.7; HRMS (ESI) m/z: $[\mathrm{M}+\mathrm{Na}]^{+} \mathrm{Calcd}$ for $\mathrm{C}_{13} \mathrm{H}_{13} \mathrm{~N}_{3} \mathrm{O}_{3} \mathrm{Na} 282.0849$; Found 282.0845.

\section{1-Methyl-3-(2-oxopropyl)-3,4-dihydroquinoxalin-2(1H)-one $(47)^{3}$}<smiles>CC(=O)CC1Nc2ccccc2N(C)C1=O</smiles>

Purified by silica gel column chromatography using the mixture of ethyl acetate and petroleum ether as eluant. Yellow solid (371 mg, 85\% yield); ${ }^{1} \mathrm{H}$ NMR (500 $\left.\mathrm{MHz}, \mathrm{CDCl}_{3}\right) \delta 6.98-6.91$ $(\mathrm{m}, 2 \mathrm{H}), 6.91-6.84(\mathrm{~m}, 1 \mathrm{H}), 6.74(\mathrm{dd}, J=7.7,1.3 \mathrm{~Hz}, 1 \mathrm{H}), 4.31(\mathrm{dd}, J=10.2,2.3 \mathrm{~Hz}, 1 \mathrm{H})$, $3.38-3.31(\mathrm{~m}, 4 \mathrm{H}), 2.83(\mathrm{dd}, J=18.5,10.2 \mathrm{~Hz}, 1 \mathrm{H}), 2.24(\mathrm{~s}, 3 \mathrm{H}) ;{ }^{13} \mathrm{C}$ NMR $(126 \mathrm{MHz}$, $\left.\mathrm{CDCl}_{3}\right) \delta 207.8,166.9,134.4,128.7,123.8,119.9,114.8,114.7,52.6,44.7,30.4,29.2$.

\section{1-Methyl-3-(2-oxopropylidene)-3,4-dihydroquinoxalin-2(1H)-one (48)}<smiles>CC(=O)C=C1Nc2ccccc2N(C)C1=O</smiles>

Purified by silica gel column chromatography using the mixture of ethyl acetate and petroleum ether as eluant. Yellow solid (52 mg, $48 \%$ yield); M.p. $152-153{ }^{\circ} \mathrm{C} .{ }^{1} \mathrm{H}$ NMR $\left(500 \mathrm{MHz}, \mathrm{CDCl}_{3}\right)$ $\delta 13.32(\mathrm{~s}, 1 \mathrm{H}), 7.17-7.15(\mathrm{~m}, 3 \mathrm{H}), 7.14-7.10(\mathrm{~m}, 1 \mathrm{H}), 6.27(\mathrm{~s}, 1 \mathrm{H}), 3.63(\mathrm{~s}, 3 \mathrm{H}), 2.27$ (s, 
$3 \mathrm{H}) ;{ }^{13} \mathrm{C}$ NMR $\left(126 \mathrm{MHz}, \mathrm{CDCl}_{3}\right) \delta 198.9,156.2,142.7,128.0,125.3,124.3,123.6,116.2$, 114.4, 94.5, 30.0, 29.8; HRMS (ESI) m/z: $[\mathrm{M}+\mathrm{Na}]^{+}$Calcd for $\mathrm{C}_{12} \mathrm{H}_{12} \mathrm{~N}_{2} \mathrm{O}_{2} \mathrm{Na}$ 239.0791; Found 239.0792 .

\section{1-Methyl-3-(3-oxobutan-2-yl)quinoxalin-2(1H)-one (50)}<smiles>CC(=O)C(C)c1nc2ccccc2n(C)c1=O</smiles>

Purified by silica gel column chromatography using the mixture of ethyl acetate and petroleum ether as eluant. White solid (115 mg, 5\% yield); M.p. $134-135{ }^{\circ} \mathrm{C} .{ }^{1} \mathrm{H}$ NMR $\left(500 \mathrm{MHz}, \mathrm{CDCl}_{3}\right)$ $\delta 7.86(\mathrm{~d}, J=8.0 \mathrm{~Hz}, 1 \mathrm{H}), 7.54(\mathrm{dd}, J=11.5,4.2 \mathrm{~Hz}, 1 \mathrm{H}), 7.34(\mathrm{t}, J=7.7 \mathrm{~Hz}, 1 \mathrm{H}), 7.30(\mathrm{~d}, J=$ $8.3 \mathrm{~Hz}, 1 \mathrm{H}), 4.37(\mathrm{q}, J=7.1 \mathrm{~Hz}, 1 \mathrm{H}), 3.68(\mathrm{~s}, 3 \mathrm{H}), 2.33(\mathrm{~s}, 3 \mathrm{H}), 1.51(\mathrm{~d}, J=7.1 \mathrm{~Hz}, 3 \mathrm{H}) ;{ }^{13} \mathrm{C}$ NMR $\left(126 \mathrm{MHz}, \mathrm{CDCl}_{3}\right) \delta 207.5,158.9,154.5,133.1,132.7,130.3,130.2,123.8,113.7,77.3$, 77.1, 76.8, 50.8, 29.2, 29.2, 13.7; HRMS (ESI) m/z: $[\mathrm{M}+\mathrm{Na}]^{+}$Calcd for $\mathrm{C}_{13} \mathrm{H}_{14} \mathrm{~N}_{2} \mathrm{O}_{2} \mathrm{Na}$ 253.0947; Found 253.0954.

1-Methyl-3-(3-oxopentan-2-yl)quinoxalin-2(1H)-one (51)<smiles>CCC(=O)C(C)c1nc2ccccc2n(C)c1=O</smiles>

Purified by silica gel column chromatography using the mixture of ethyl acetate and petroleum ether as eluant. White solid (34 mg, 28\% yield); M.p. $142-143{ }^{\circ} \mathrm{C} .{ }^{1} \mathrm{H}$ NMR (500 MHz, $\mathrm{CDCl}_{3}$ ) $\delta 7.88(\mathrm{dd}, J=8.0,1.4 \mathrm{~Hz}, 1 \mathrm{H}), 7.55(\mathrm{ddd}, J=8.6,7.4,1.5 \mathrm{~Hz}, 1 \mathrm{H}), 7.37-7.34(\mathrm{~m}, 1 \mathrm{H}), 7.33$ $-7.30(\mathrm{~m}, 1 \mathrm{H}), 4.40(\mathrm{q}, J=7.1 \mathrm{~Hz}, 1 \mathrm{H}), 3.69(\mathrm{~s}, 3 \mathrm{H}), 2.71(\mathrm{dd}, J=35.1,7.3 \mathrm{~Hz}, 2 \mathrm{H}), 1.52(\mathrm{~d}$, $J=7.1 \mathrm{~Hz}, 3 \mathrm{H}), 1.11(\mathrm{t}, J=7.3 \mathrm{~Hz}, 3 \mathrm{H}) ;{ }^{13} \mathrm{C} \mathrm{NMR}\left(126 \mathrm{MHz}, \mathrm{CDCl}_{3}\right) \delta 210.2,159.0,154.6$, $133.1,132.7,130.3,130.2,123.7,113.6,50.0,35.1,29.2,13.8,7.9 ;$ HRMS (ESI) m/z: $[\mathrm{M}+\mathrm{Na}]^{+}$Calcd for $\mathrm{C}_{14} \mathrm{H}_{16} \mathrm{~N}_{2} \mathrm{O}_{2} \mathrm{Na}$ 267.1104; Found 267.1104. 


\section{Computational Details}

To explore the stabilization of $\mathrm{E}$ isomer and $\mathrm{Z}$ isomer, density functional theory (DFT) calculations with wB97X-D ${ }^{4}$ were carried out at wB97xd/def2TZVPP/SMD(acetone)//wB97xd/6-31G(d) levels of theory with Gaussian 16 package. On the basis of our calculation, E-isomer is more stable than Z-isomer by 1.2 $\mathrm{kcal} / \mathrm{mol}$. The computational results are consistent with the experimental observations.

\section{Cartesian coordinates of the optimized structures}

$E$

\begin{tabular}{|c|c|c|c|}
\hline $\mathrm{C}$ & -4.26936200 & -0.19605400 & 0.21188700 \\
\hline $\mathrm{C}$ & -3.33819600 & 0.81630800 & 0.04801200 \\
\hline $\mathrm{C}$ & -1.97236700 & 0.50493600 & -0.01121800 \\
\hline $\mathrm{C}$ & -1.57123600 & -0.84002400 & 0.10559800 \\
\hline $\mathrm{C}$ & -2.52987700 & -1.84776000 & 0.26902500 \\
\hline $\mathrm{C}$ & -3.87382700 & -1.53346400 & 0.32029400 \\
\hline $\mathrm{H}$ & -5.32322700 & 0.06040700 & 0.25477800 \\
\hline $\mathrm{H}$ & -3.67337500 & 1.84264800 & -0.03842900 \\
\hline $\mathrm{H}$ & -2.17892000 & -2.87038800 & 0.36180500 \\
\hline $\mathrm{H}$ & -4.61511700 & -2.31464600 & 0.44843100 \\
\hline $\mathrm{C}$ & 0.66523000 & -0.27333600 & -0.03490900 \\
\hline $\mathrm{N}$ & -0.23337100 & -1.19288200 & 0.09943800 \\
\hline $\mathrm{N}$ & -0.99738900 & 1.47554300 & -0.20817500 \\
\hline $\mathrm{C}$ & -1.37265400 & 2.86915200 & -0.40647900 \\
\hline $\mathrm{H}$ & -1.99982700 & 2.97439800 & -1.29684200 \\
\hline $\mathrm{H}$ & -1.91148300 & 3.23907500 & 0.46987700 \\
\hline $\mathrm{H}$ & -0.45466900 & 3.43729100 & -0.54234800 \\
\hline
\end{tabular}




\begin{tabular}{|c|c|c|c|}
\hline $\mathrm{C}$ & 0.34164700 & 1.14904100 & -0.34177600 \\
\hline $\mathrm{O}$ & 1.18465400 & 1.94594400 & -0.71400300 \\
\hline $\mathrm{C}$ & 2.09357900 & -0.64465800 & -0.03150700 \\
\hline $\mathrm{C}$ & 3.07458900 & 0.31650700 & 0.59181900 \\
\hline $\mathrm{C}$ & 4.44956100 & 0.40764300 & -0.00693200 \\
\hline $\mathrm{H}$ & 4.85503000 & -0.58789900 & -0.20473200 \\
\hline $\mathrm{H}$ & 4.36962500 & 0.92999600 & -0.96734900 \\
\hline $\mathrm{H}$ & 5.10293500 & 0.97040000 & 0.66125200 \\
\hline $\mathrm{N}$ & 2.59018700 & -1.77739900 & -0.40188100 \\
\hline $\mathrm{O}$ & 2.71301300 & 0.94656800 & 1.56262200 \\
\hline $\mathrm{O}$ & 1.76338600 & -2.77502200 & -0.76585800 \\
\hline $\mathrm{H}$ & 0.84208400 & -2.52777500 & -0.51709100 \\
\hline \multicolumn{4}{|c|}{$Z$} \\
\hline $\mathrm{C}$ & -4.38377500 & -0.38853600 & 0.14072400 \\
\hline $\mathrm{C}$ & -3.48530200 & 0.65341000 & -0.02129700 \\
\hline $\mathrm{C}$ & -2.10985400 & 0.38576400 & -0.06204100 \\
\hline $\mathrm{C}$ & -1.65911800 & -0.94325600 & 0.06413300 \\
\hline $\mathrm{C}$ & -2.58854800 & -1.97896800 & 0.22886100 \\
\hline $\mathrm{C}$ & -3.94303900 & -1.71026100 & 0.26587600 \\
\hline $\mathrm{H}$ & -5.44630400 & -0.16775300 & 0.17058000 \\
\hline $\mathrm{H}$ & -3.85334900 & 1.66799200 & -0.11570400 \\
\hline $\mathrm{H}$ & -2.19934800 & -2.98697200 & 0.32548300 \\
\hline $\mathrm{H}$ & -4.65904900 & -2.51514200 & 0.39313600 \\
\hline $\mathrm{C}$ & 0.53872900 & -0.32052800 & -0.10586500 \\
\hline
\end{tabular}




\begin{tabular}{|c|c|c|c|}
\hline $\mathrm{N}$ & -0.31475300 & -1.26963100 & 0.04764500 \\
\hline $\mathrm{N}$ & -1.16367200 & 1.39138300 & -0.22899000 \\
\hline $\mathrm{C}$ & -1.57664700 & 2.77955900 & -0.37798200 \\
\hline $\mathrm{H}$ & -2.20989800 & 2.89521300 & -1.26247200 \\
\hline $\mathrm{H}$ & -2.12372200 & 3.10736700 & 0.51043200 \\
\hline $\mathrm{H}$ & -0.67439100 & 3.37659600 & -0.49402400 \\
\hline $\mathrm{C}$ & 0.18628700 & 1.10981200 & -0.28668500 \\
\hline $\mathrm{O}$ & 1.03730300 & 1.96772000 & -0.48414000 \\
\hline $\mathrm{C}$ & 1.99368100 & -0.61858500 & -0.13112100 \\
\hline $\mathrm{C}$ & 2.89897400 & 0.27322800 & 0.71181600 \\
\hline $\mathrm{C}$ & 4.06154400 & 0.95555400 & 0.04855900 \\
\hline $\mathrm{H}$ & 4.67579700 & 0.23535700 & -0.49618100 \\
\hline $\mathrm{H}$ & 3.65687800 & 1.67769000 & -0.66825300 \\
\hline $\mathrm{H}$ & 4.65306300 & 1.47669100 & 0.80347600 \\
\hline $\mathrm{N}$ & 2.34373600 & -1.66916000 & -0.76942000 \\
\hline $\mathrm{O}$ & 2.60567500 & 0.41133200 & 1.87830500 \\
\hline $\mathrm{O}$ & 3.69736600 & -1.92821900 & -0.66442100 \\
\hline $\mathrm{H}$ & 3.80281400 & -2.72500500 & -1.19892400 \\
\hline
\end{tabular}

\section{References}

1. (a) Xie, L.-Y.; Bai, Y.-S.; Xu, X.-Q.; Peng, X.; Tang, H.-S.; Huang, Y.; Lin, Y.-W.; Cao, Z.; He, W.-M. Green Chem. 2020, 22, 1720; (b) Wang, J.; Sun, B.; Zhang, L.; Xu, T.; Xie, Y.; Jin, C. Org. Chem. Front. 2020, 7, 113; (c) Bao, P.; Liu, F.; Lv, Y.; Yue, H.; Li, J.-S.; Wei, W. Org. Chem. Front. 2020, 7, 492.

2. Wang, J.; Sun, B.; Zhang, L.; Xu, T.; Xie, Y.; Jin, C. Org. Chem. Front. 2020, 7, 113.

3. Rostoll-Berenguer, J.; Blay, G.; Muñoz, M. C.; Pedro, J. R.; Vila, C. Org. Lett. 2019, 21, 6011.

4. Chai, J. D.; Head-Gordon, M. Phys. Chem. Chem. Phys. 2008, 10, 6615. 
6. X-ray Crystal Data for 18 and 20

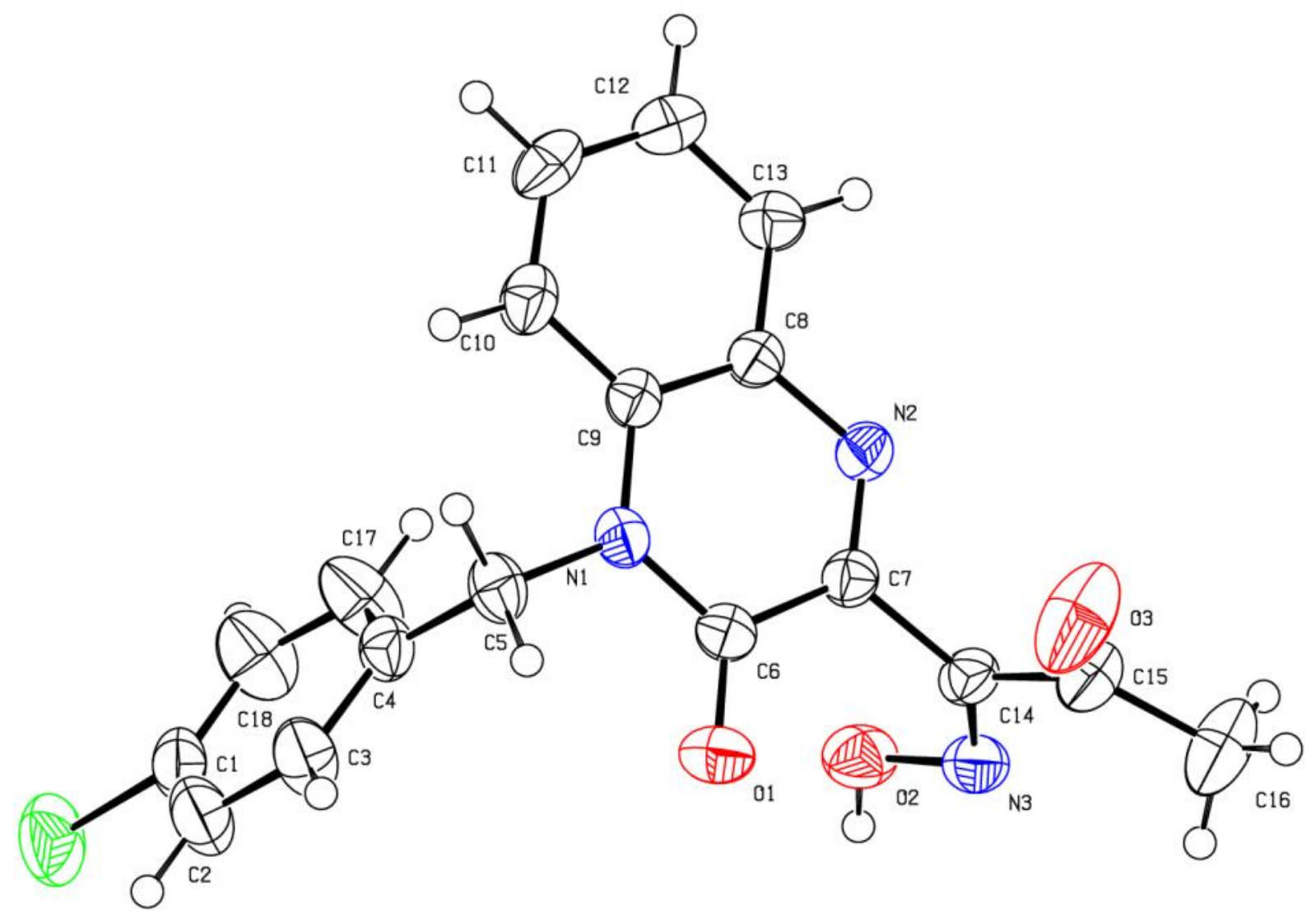

Figure S2 Single-crystal X-ray structure of 18. Ellipsoids are represented at 30\% probability. 
Table S1. Crystallographic data and structure refinement for $\mathbf{1 8}$

\begin{tabular}{|c|c|}
\hline CCDC & 2007883 \\
\hline Empirical formula & $\mathrm{C}_{18} \mathrm{H}_{14} \mathrm{ClN}_{3} \mathrm{O}_{3}$ \\
\hline Formula weight & 355.77 \\
\hline Temperature, $\mathrm{K}$ & 100.15 \\
\hline Wavelength, $\AA$ & 0.71073 \\
\hline Crystal system & Triclinic \\
\hline Space group & $\mathrm{P}-1$ \\
\hline$a, b, c, \AA$ & 8.7090(11), 9.9399(12), 10.3203(12) \\
\hline$\alpha, \beta, \gamma,{ }^{\mathrm{o}}$ & $107.538(2), 94.549(2), 100.768(2)$ \\
\hline Volume, $\AA^{3}$ & $828.05(17)$ \\
\hline$Z$ & 2 \\
\hline Calculated density, $\mathrm{Mg} / \mathrm{m}^{\wedge} 3$ & 1.427 \\
\hline Absorption coefficient, $\mathrm{mm}^{-1}$ & 0.254 \\
\hline$F(000)$ & 368 \\
\hline Theta range for data collection, ${ }^{\circ}$ & 2.406 to 27.167 \\
\hline Limiting indices & $-10<=\mathrm{h}<=10,-12<=\mathrm{k}<=6,-9<=1<=13$ \\
\hline Reflections collected / unique & $5013 / 3602[\mathrm{R}(\mathrm{int})=0.0147]$ \\
\hline Absorption correction & Semi-empirical from equivalents \\
\hline Refinement method & Full-matrix least-squares on $\mathrm{F}^{2}$ \\
\hline Data / restraints / parameters & 3602 / 0 / 227 \\
\hline Goodness of fit on $F^{2}$ & 1.027 \\
\hline Final $R$ indices [I>2sigma(I)] & $\mathrm{R} 1=0.0461, \mathrm{wR} 2=0.1013$ \\
\hline $\mathrm{R}$ indices (all data) & $\mathrm{R} 1=0.0714, \mathrm{wR} 2=0.1129$ \\
\hline
\end{tabular}



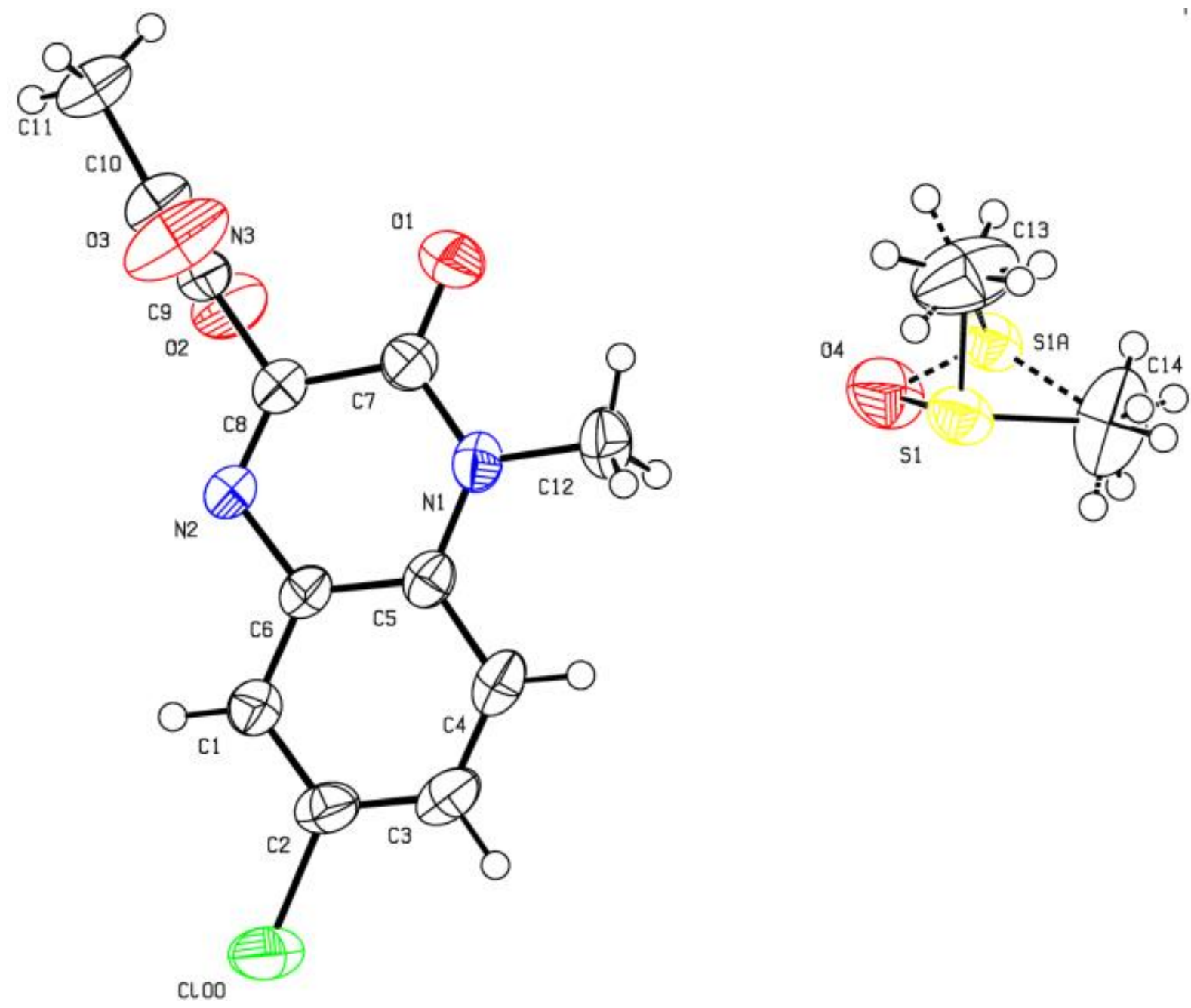

Figure S3 Single-crystal X-ray structure of 20. Ellipsoids are represented at 30\% probability. 
Table S2. Crystallographic data and structure refinement for $\mathbf{2 0}$

\begin{tabular}{|c|c|}
\hline CCDC & 2009728 \\
\hline Empirical formula & $\mathrm{C}_{14} \mathrm{H}_{16} \mathrm{~N}_{3} \mathrm{O}_{4} \mathrm{SCl}$ \\
\hline Formula weight & 357.81 \\
\hline Temperature, $\mathrm{K}$ & 296.15 \\
\hline Wavelength, $\AA$ & 0.71073 \\
\hline Crystal system & Monoclinic \\
\hline Space group & $\mathrm{P} 2{ }_{1} / \mathrm{c}$ \\
\hline$a, b, c, \AA$ & 14.1459(12), 9.0935(8), 13.1492(11) \\
\hline$\alpha, \beta, \gamma,{ }^{\mathrm{o}}$ & 90, 99.0130(10), 90 \\
\hline Volume, $\AA^{3}$ & $1670.6(2)$ \\
\hline$Z$ & 4 \\
\hline Calculated density, $\mathrm{Mg} / \mathrm{m}^{\wedge} 3$ & 1.423 \\
\hline Absorption coefficient, $\mathrm{mm}^{-1}$ & 0.376 \\
\hline$F(000)$ & 744.0 \\
\hline Theta range for data collection, ${ }^{\circ}$ & 5.346 to 50.01 \\
\hline Limiting indices & $-16 \leqslant h \leqslant 16,-6 \leqslant k \leqslant 10,-15 \leqslant 1 \leqslant 15$ \\
\hline Reflections collected / unique & $8221 / 2934[$ Rint $=0.0210]$ \\
\hline Absorption correction & Semi-empirical from equivalents \\
\hline Refinement method & Full-matrix least-squares on $\mathrm{F}^{2}$ \\
\hline Data / restraints / parameters & $2934 / 0 / 215$ \\
\hline Goodness of fit on $F^{2}$ & 1.026 \\
\hline Final $R$ indices [I>2sigma(I)] & $\mathrm{R} 1=0.0402, \mathrm{wR} 2=0.1058$ \\
\hline $\mathrm{R}$ indices (all data) & $\mathrm{R} 1=0.0524, \mathrm{wR} 2=0.1152$ \\
\hline
\end{tabular}




\section{Copies of ${ }^{1} \mathrm{H},{ }^{13} \mathrm{C}$ and ${ }^{19} \mathrm{~F}$ NMR Spectra}

\section{$3{ }^{1} \mathrm{H}$ NMR (500 MHz, DMSO)}

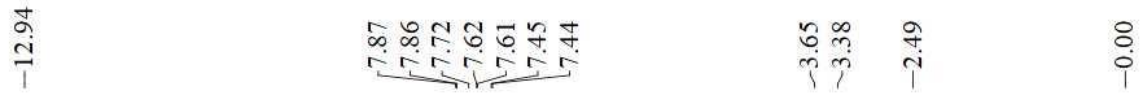<smiles>CC(=O)/C(=N/O)c1nc2ccccc2n(C)c1=O</smiles>

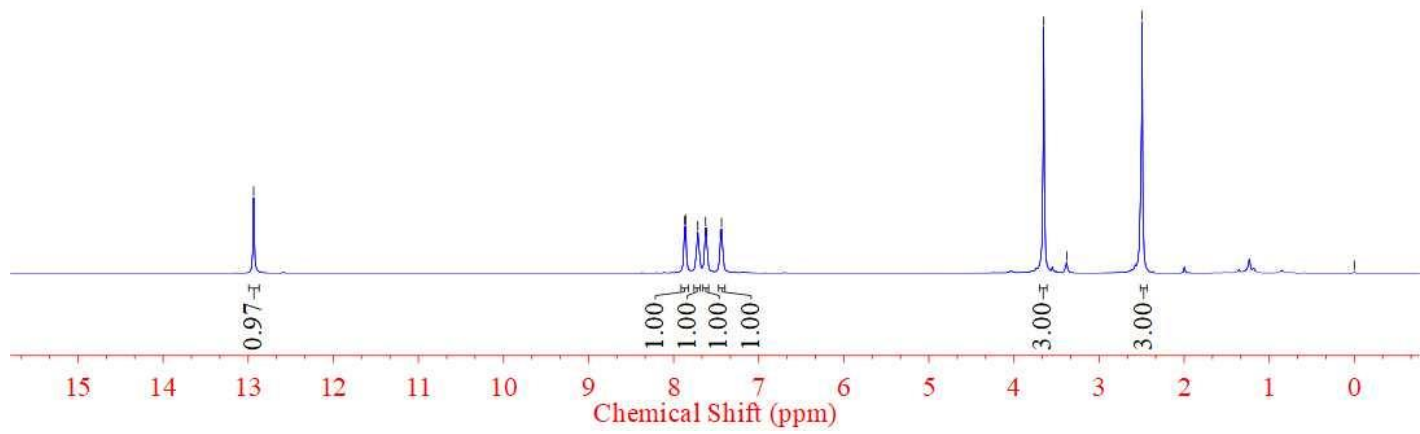

\section{$3{ }^{13} \mathrm{C}$ NMR (126 MHz, DMSO)}

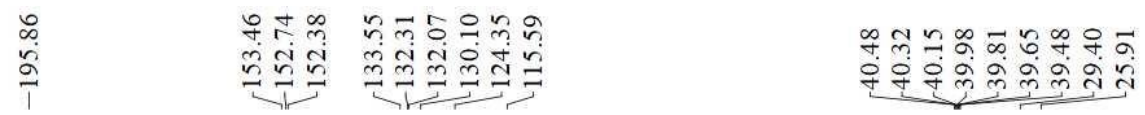<smiles>CC(=O)/C(=N/O)c1nc2ccccc2n(C)c1=O</smiles>

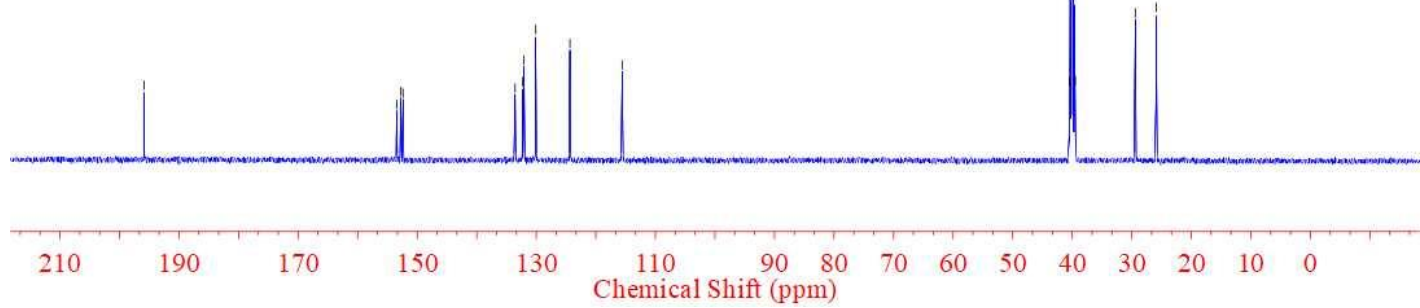




\section{$4{ }^{1}$ H NMR (500 MHz, DMSO)}
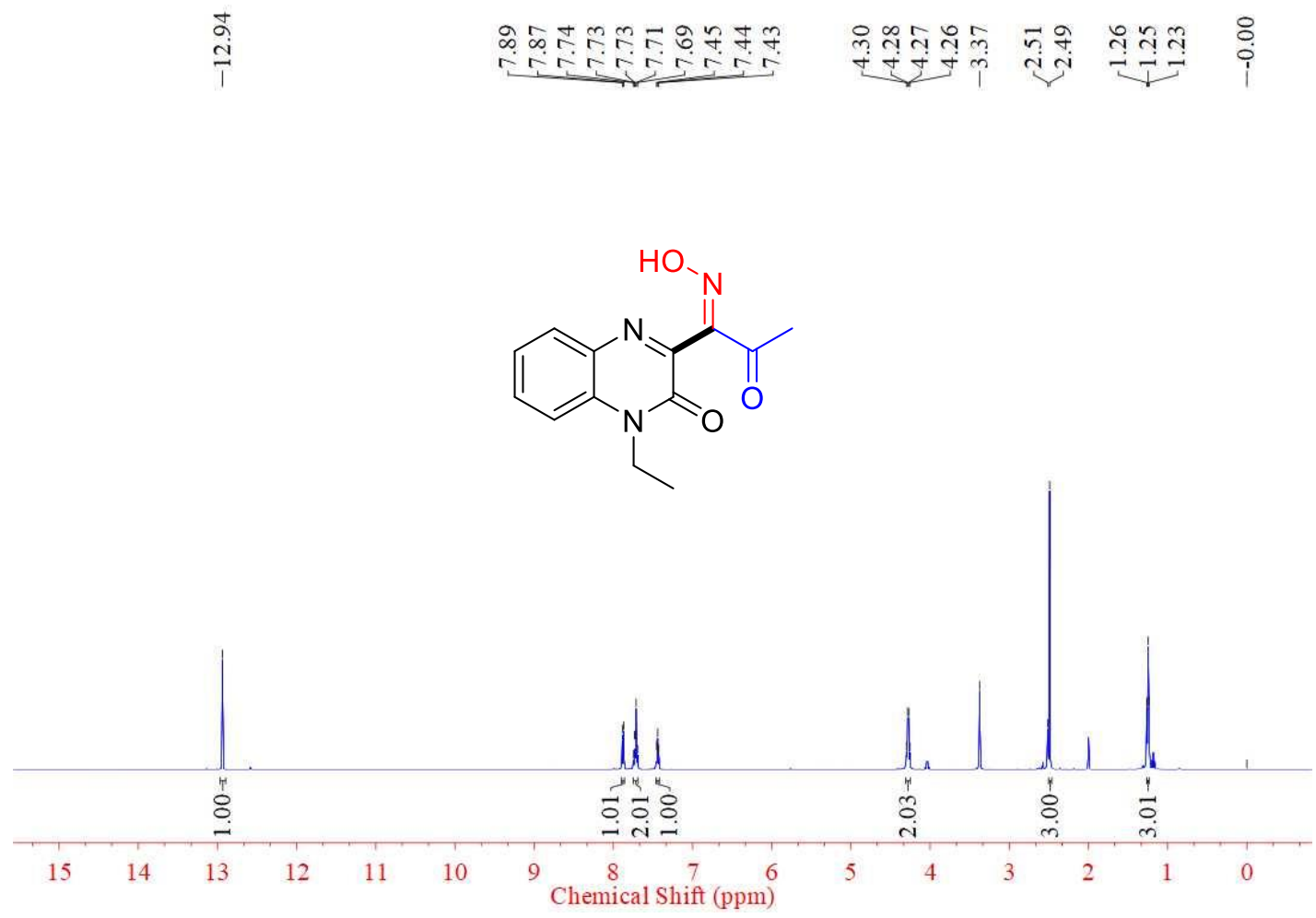

$4{ }^{13}$ C NMR (126 MHz, DMSO)

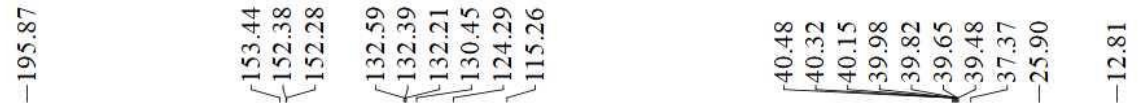

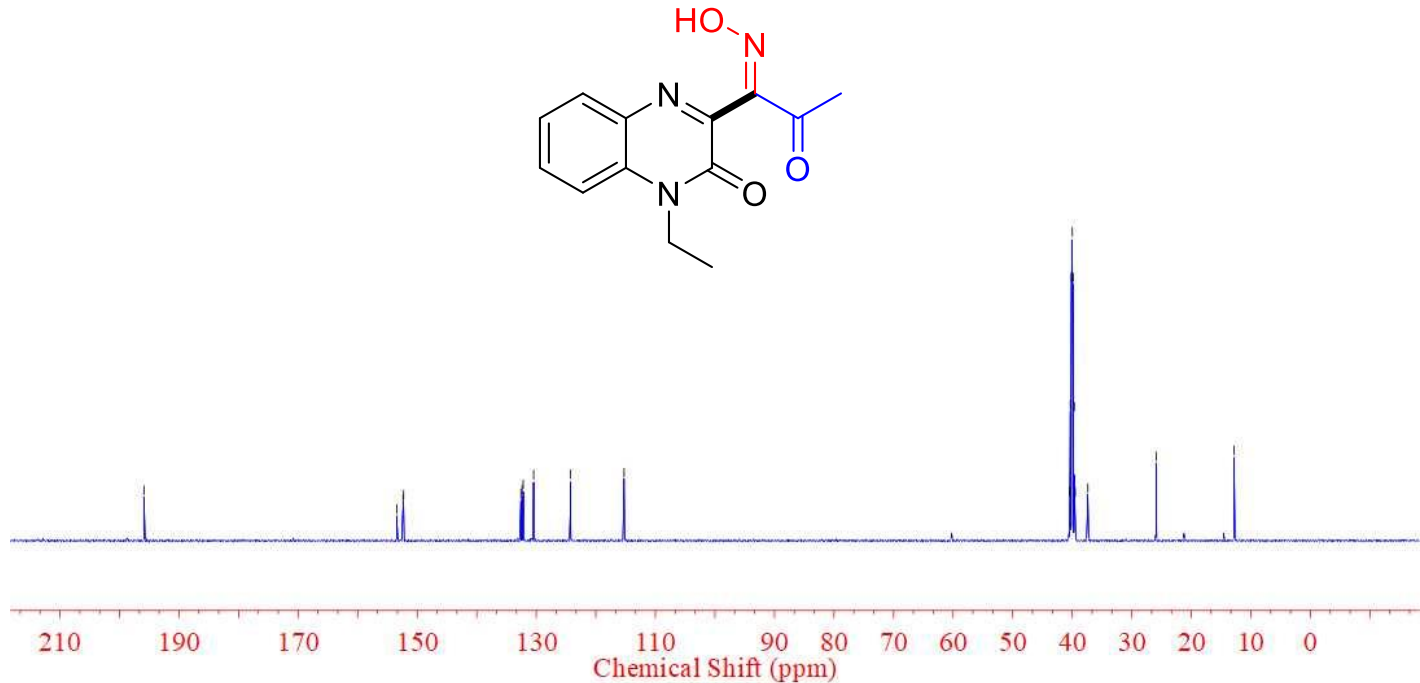




\section{$5{ }^{1}$ H NMR (500 MHz, DMSO)}
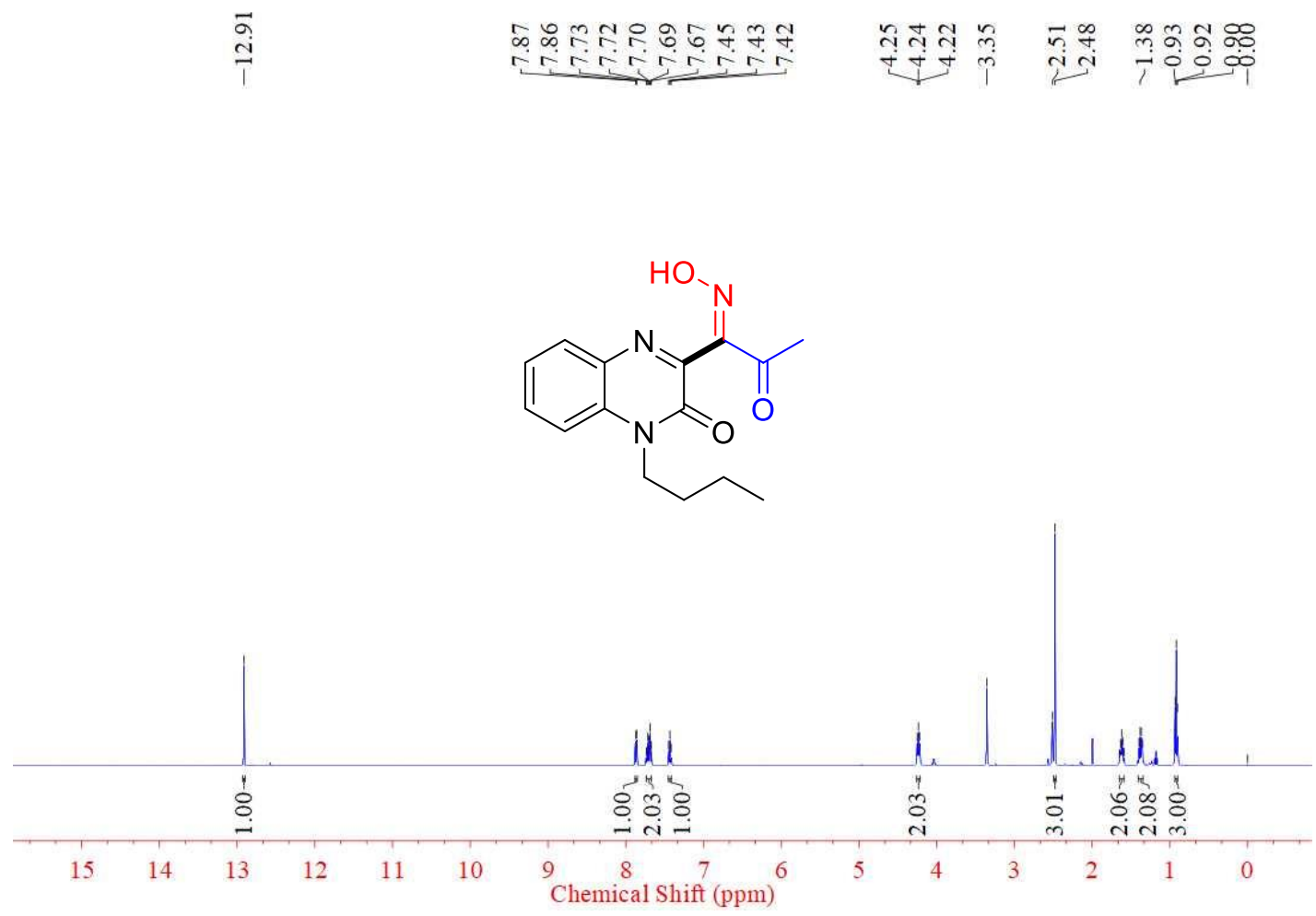

\section{$5{ }^{13} \mathrm{C}$ NMR (126 MHz, DMSO)}
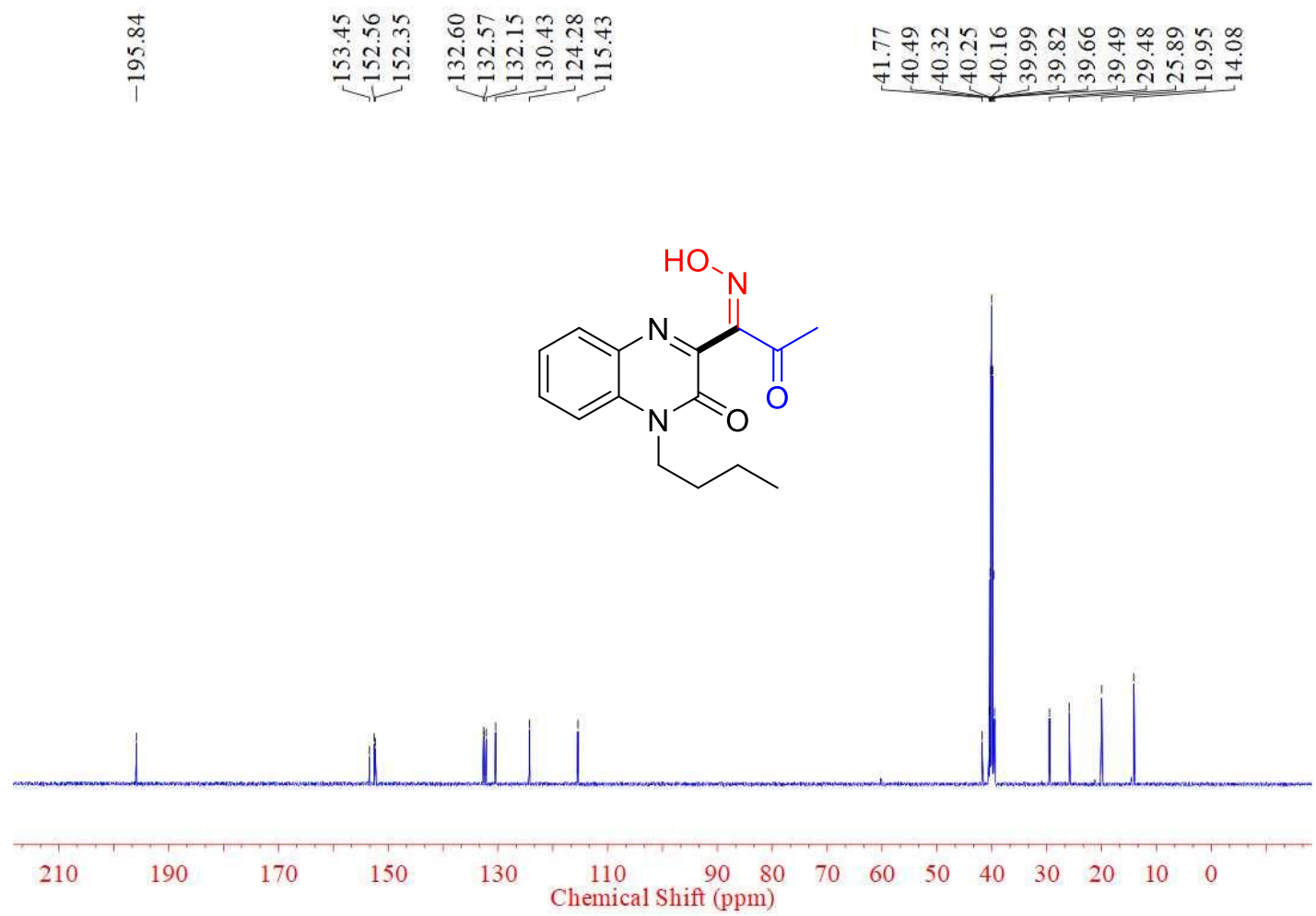
$6{ }^{1} \mathrm{H}$ NMR (500 MHz, DMSO)

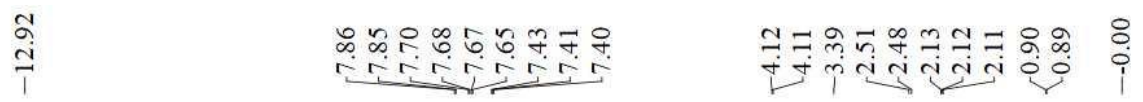<smiles>CC(=O)/C(=N/O)c1nc2ccccc2n(CC(C)C)c1=O</smiles>

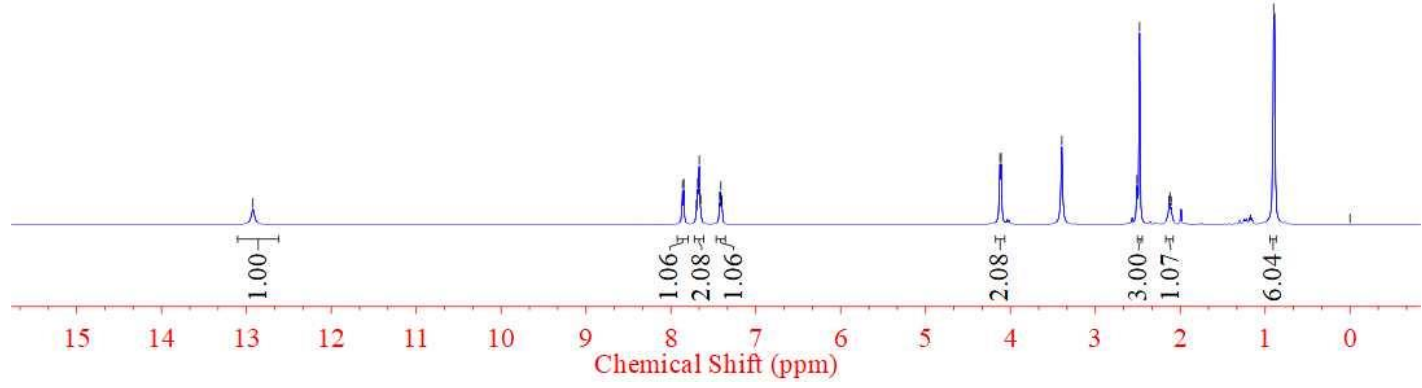

$6{ }^{13} \mathrm{C}$ NMR (126 MHz, DMSO)

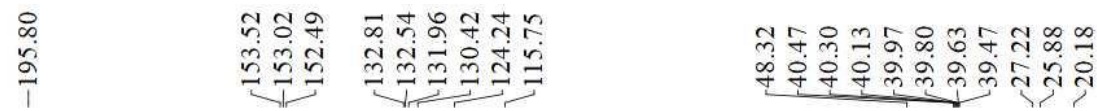

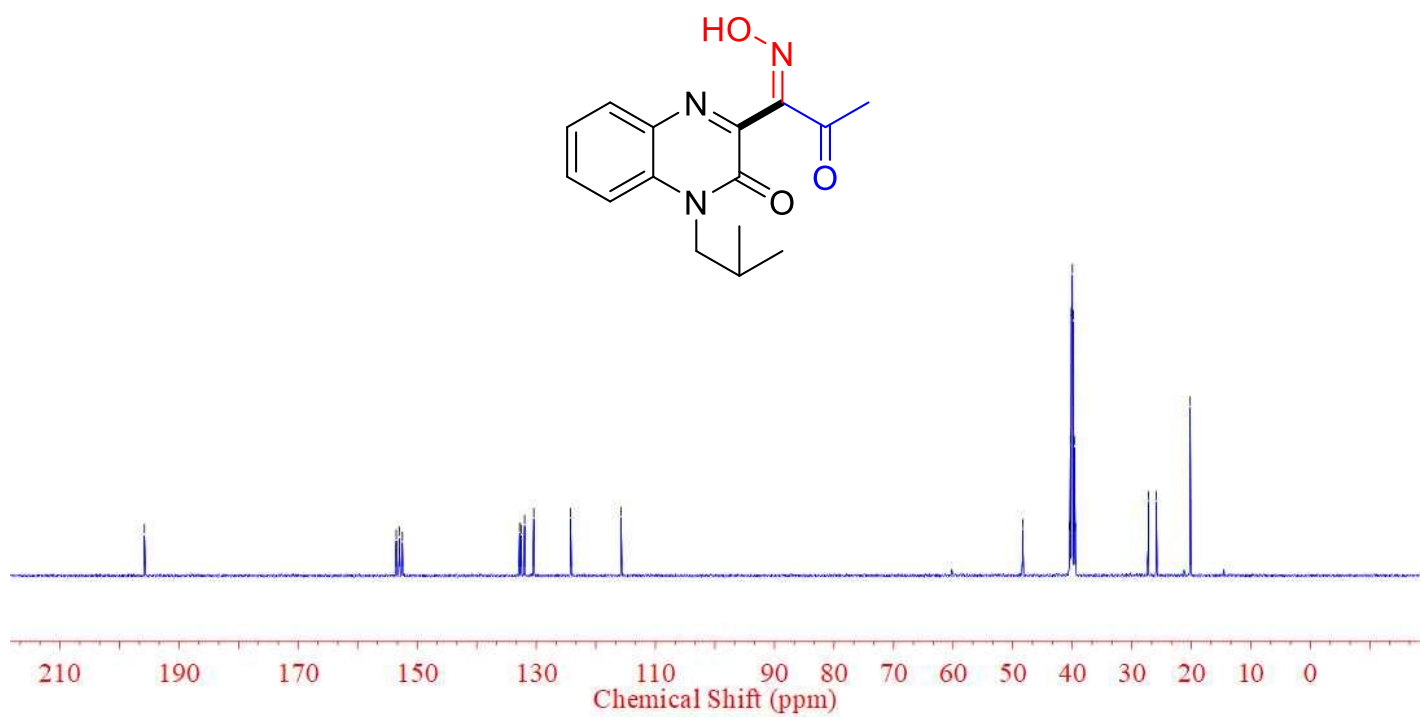




\section{$7{ }^{1}$ H NMR (500 MHz, DMSO)}
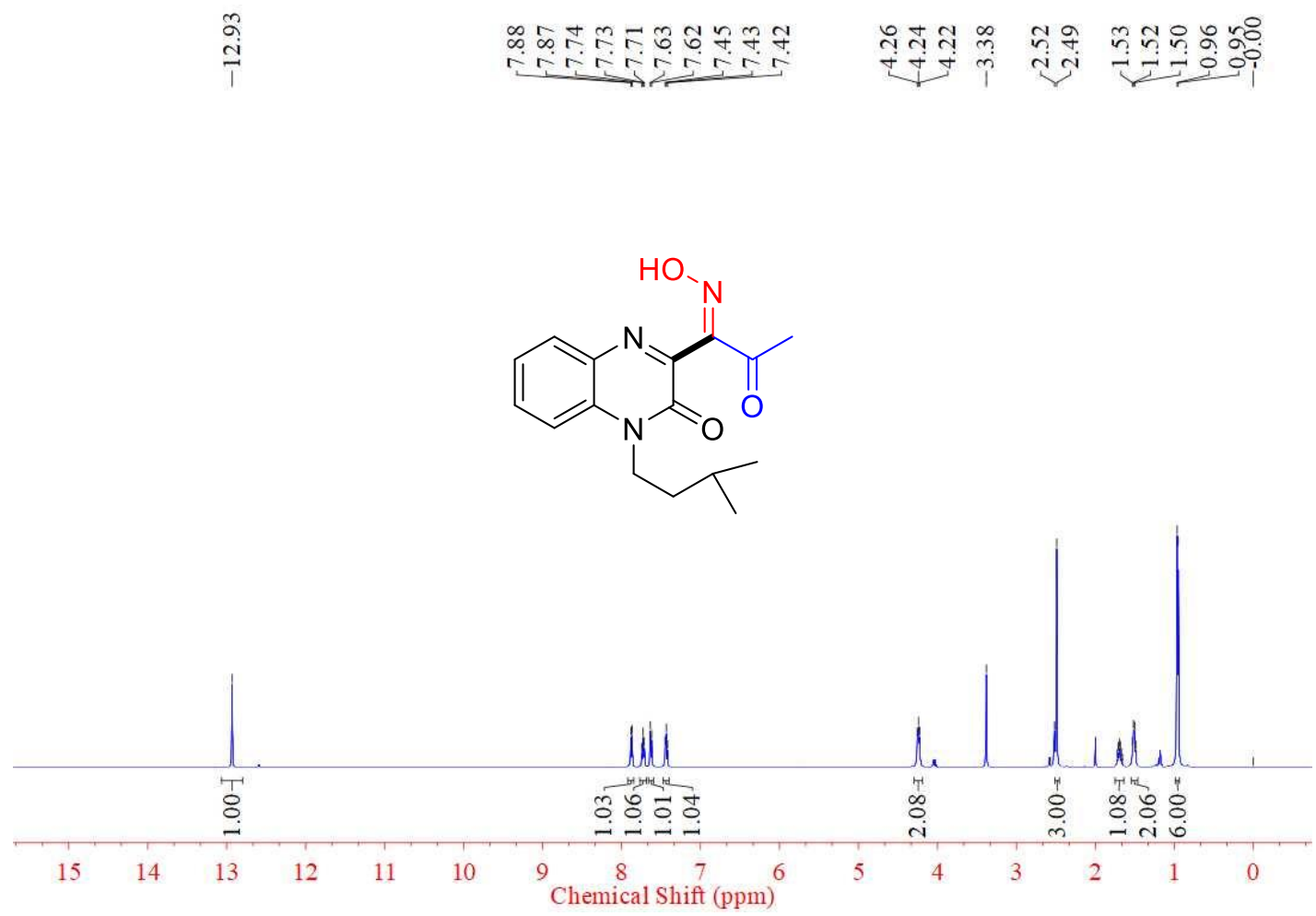

\section{$7{ }^{13} \mathrm{C}$ NMR (126 MHz, DMSO)}
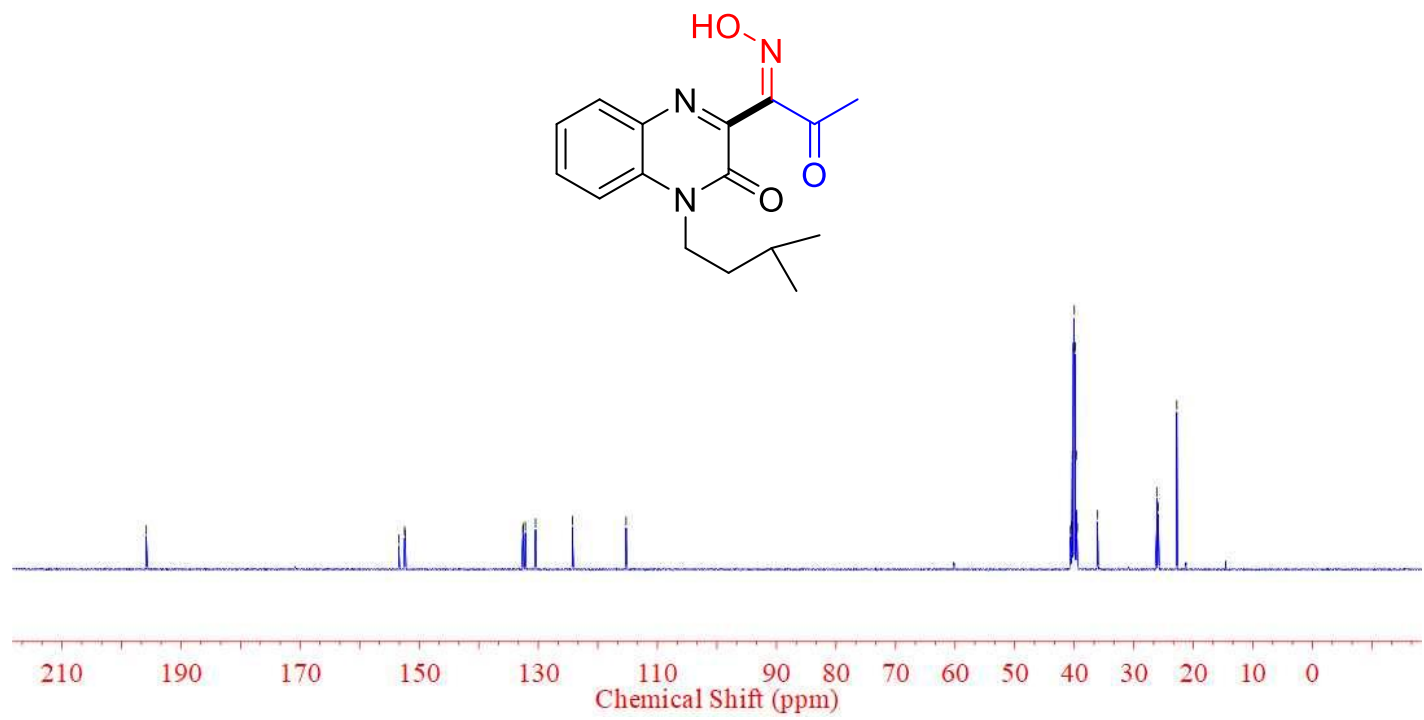


\section{$8{ }^{1}$ H NMR (500 MHz, DMSO)}
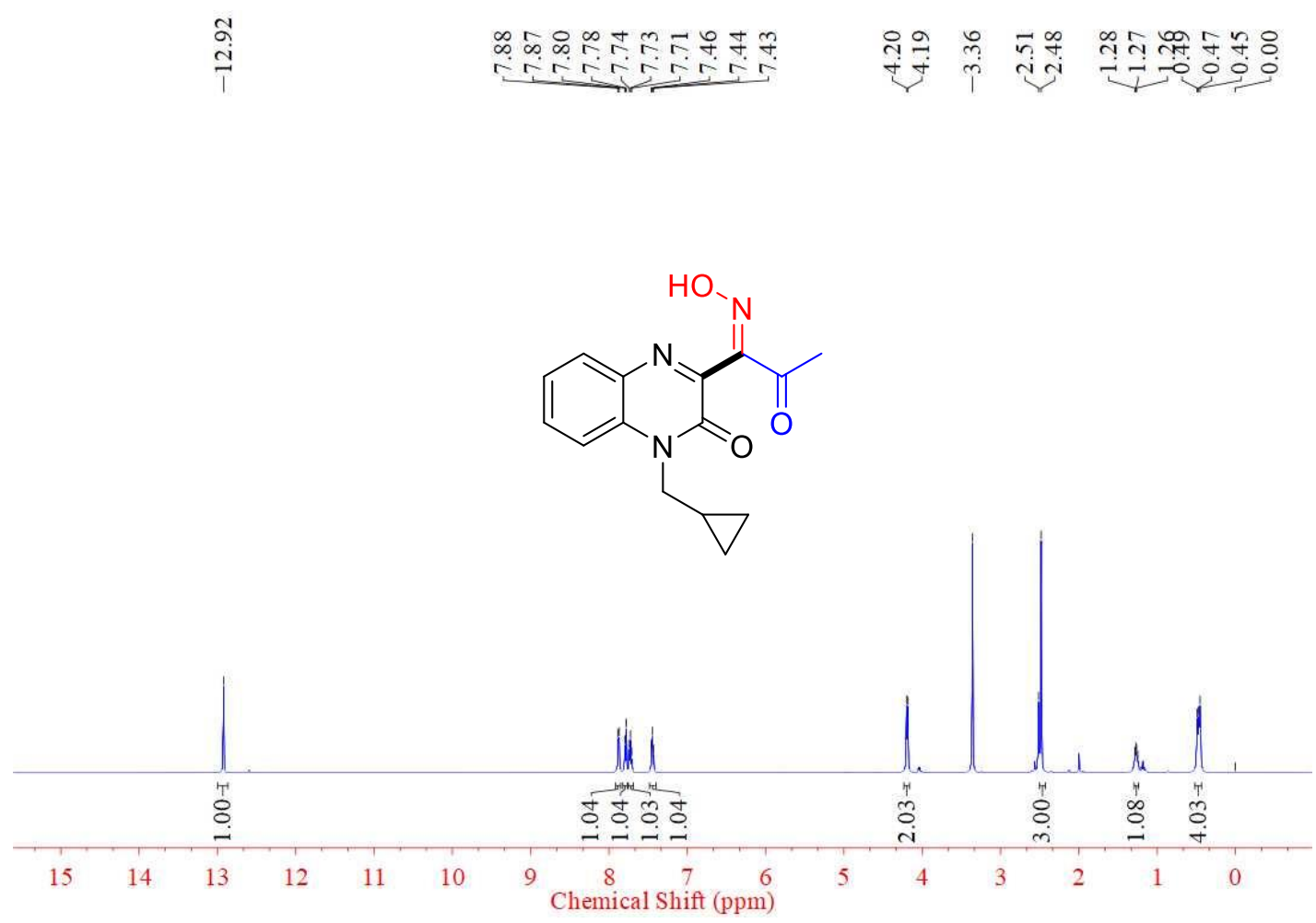

\section{$8{ }^{13} \mathrm{C}$ NMR (126 MHz, DMSO)}

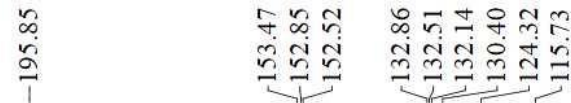

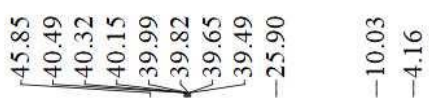<smiles>CC(=O)/C(=N/O)c1nc2ccccc2n(CC2CC2)c1=O</smiles>

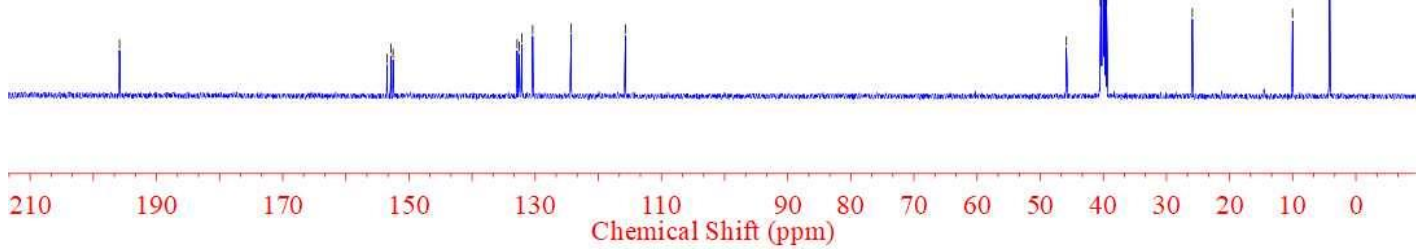


$9{ }^{1}$ H NMR (500 MHz, DMSO)
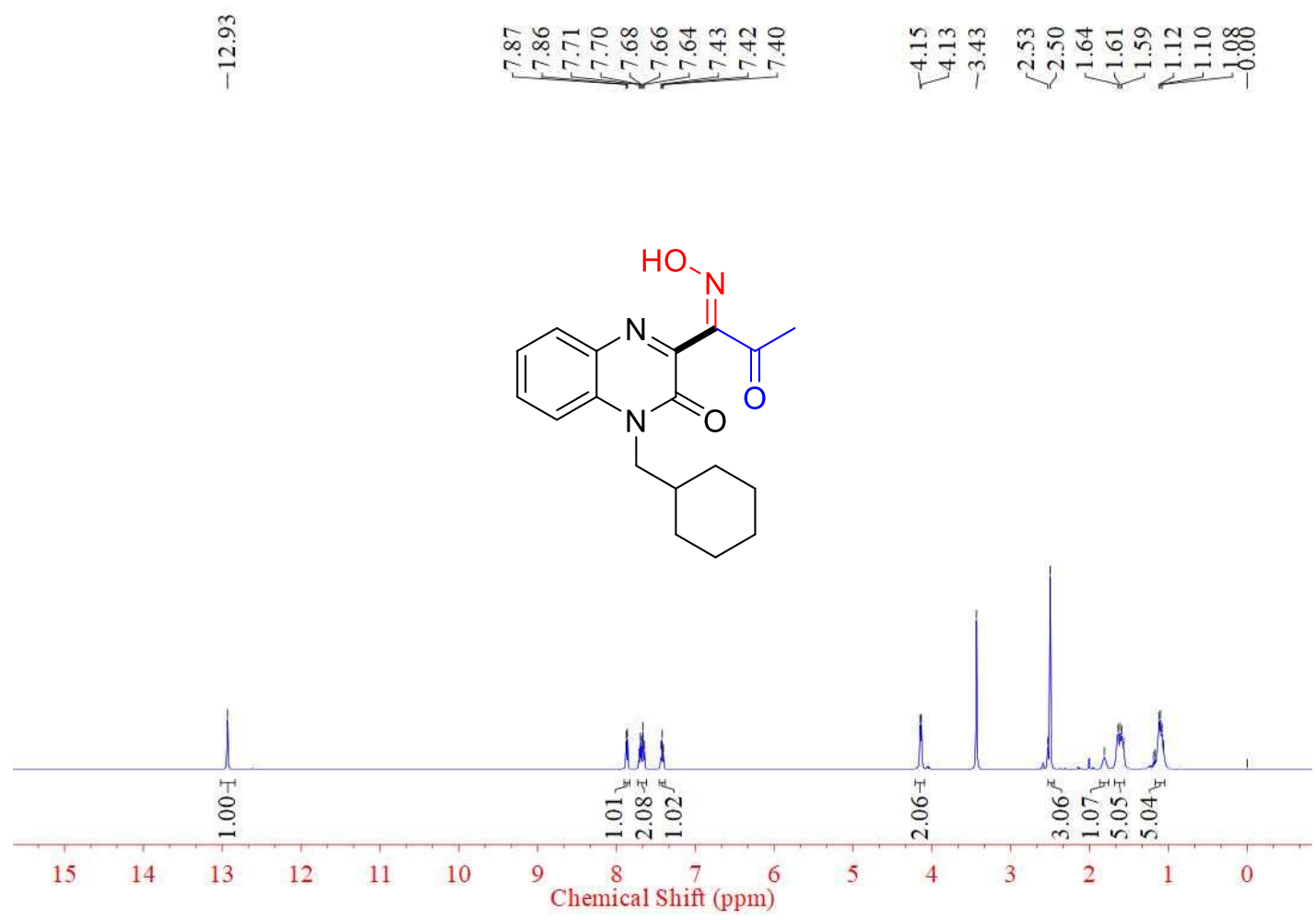

$9{ }^{13}$ C NMR (126 MHz, DMSO)

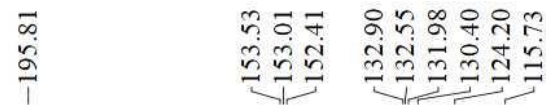

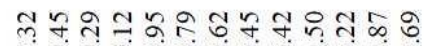

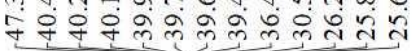

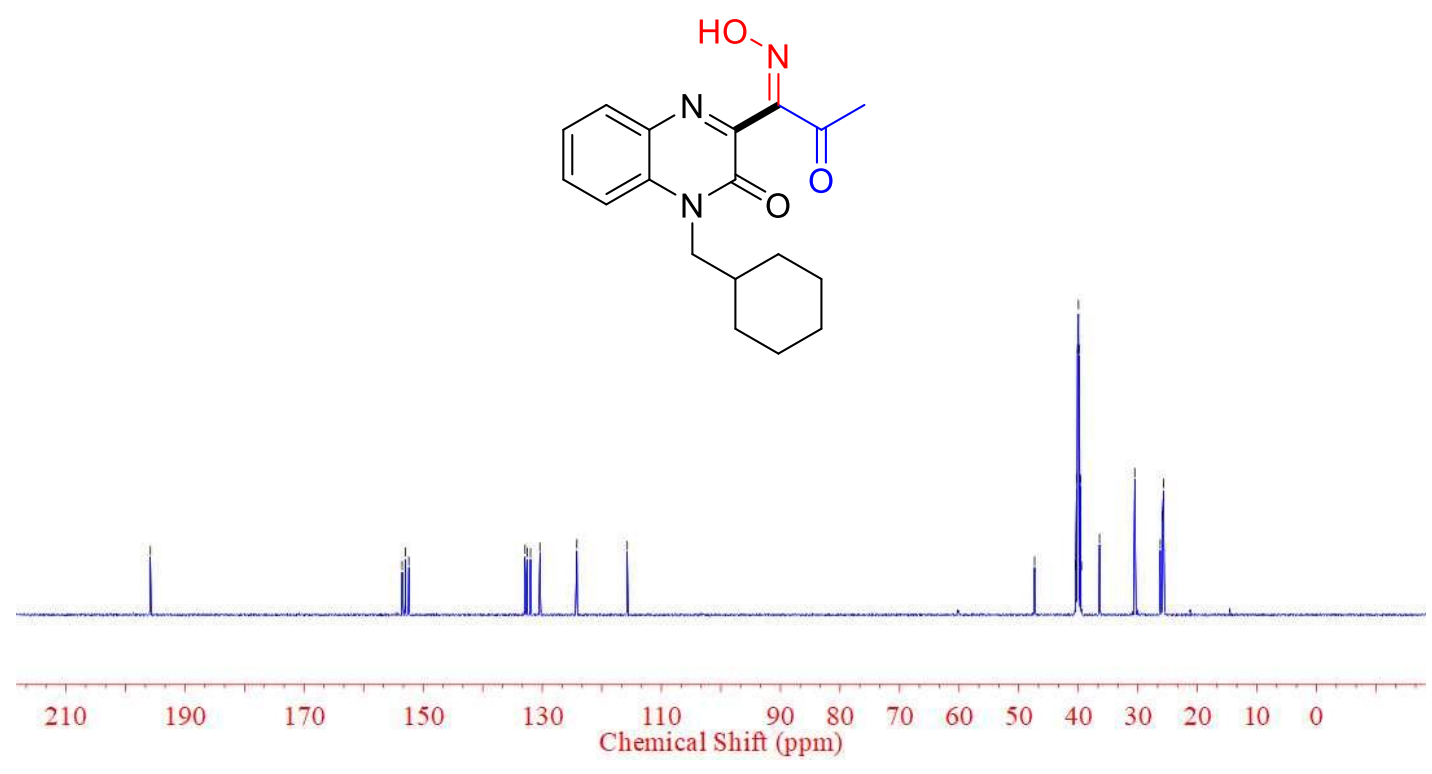




\section{$10{ }^{1}$ H NMR (500 MHz, DMSO)}
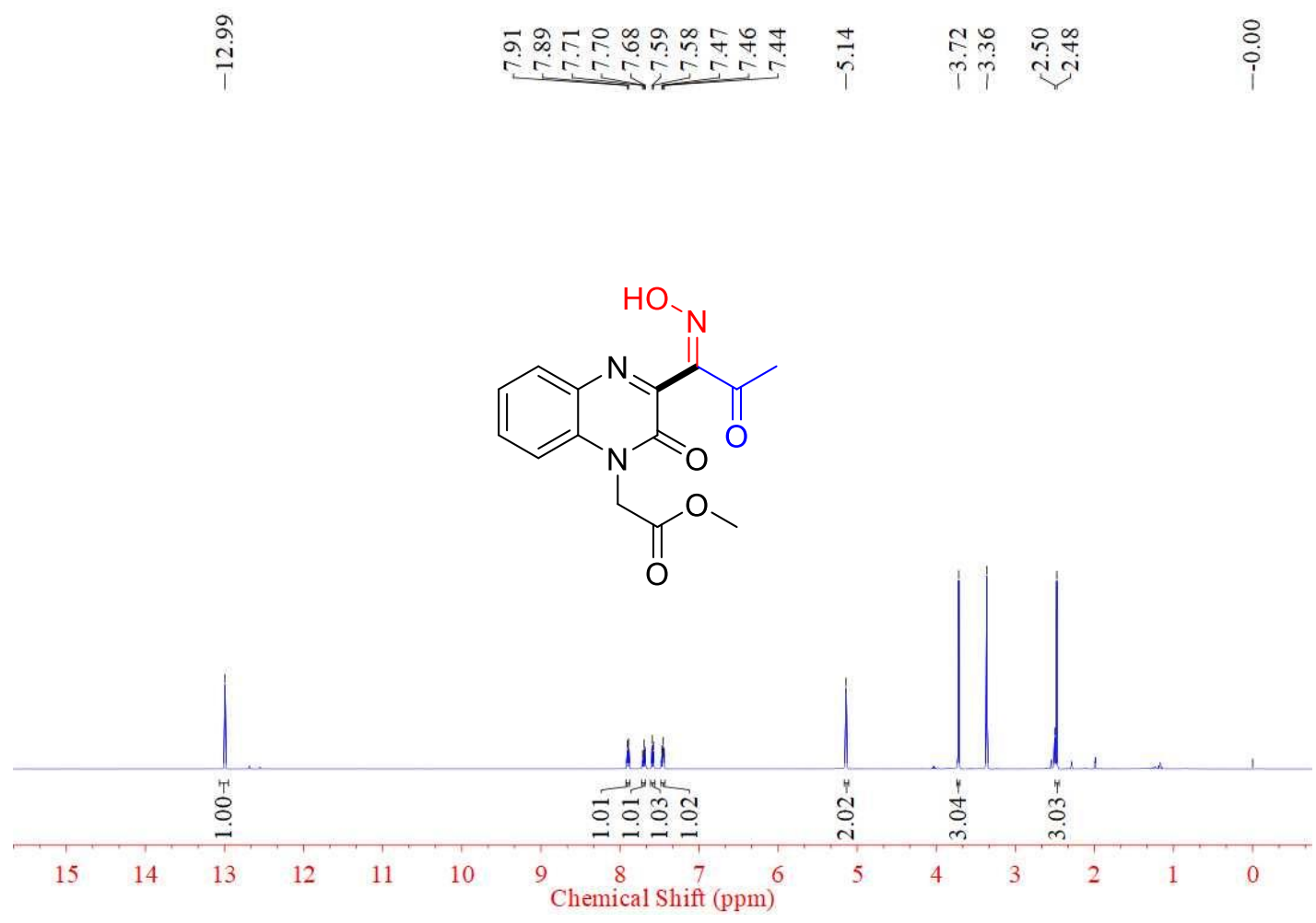

\section{$10{ }^{13} \mathrm{C}$ NMR (126 MHz, DMSO)}

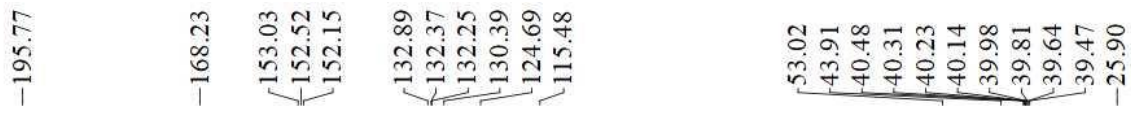<smiles>COC(=O)Cn1c(=O)c(/C(=N\O)C(C)=O)nc2ccccc21</smiles>

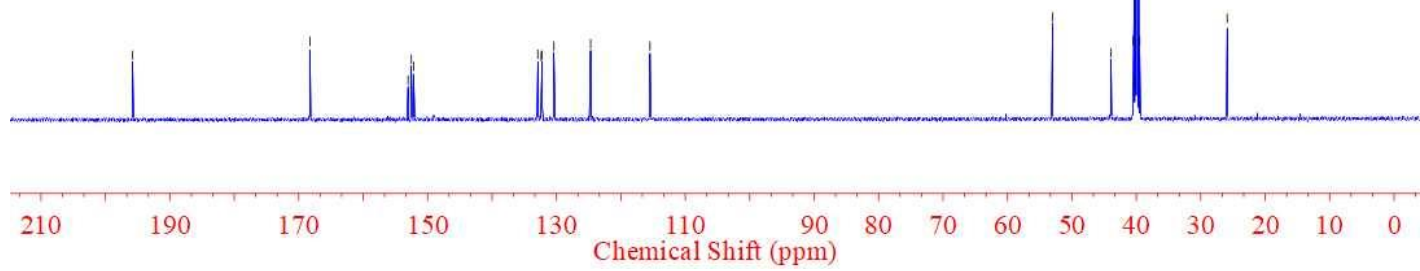




\section{$11{ }^{1} \mathrm{H}$ NMR (500 MHz, DMSO)}
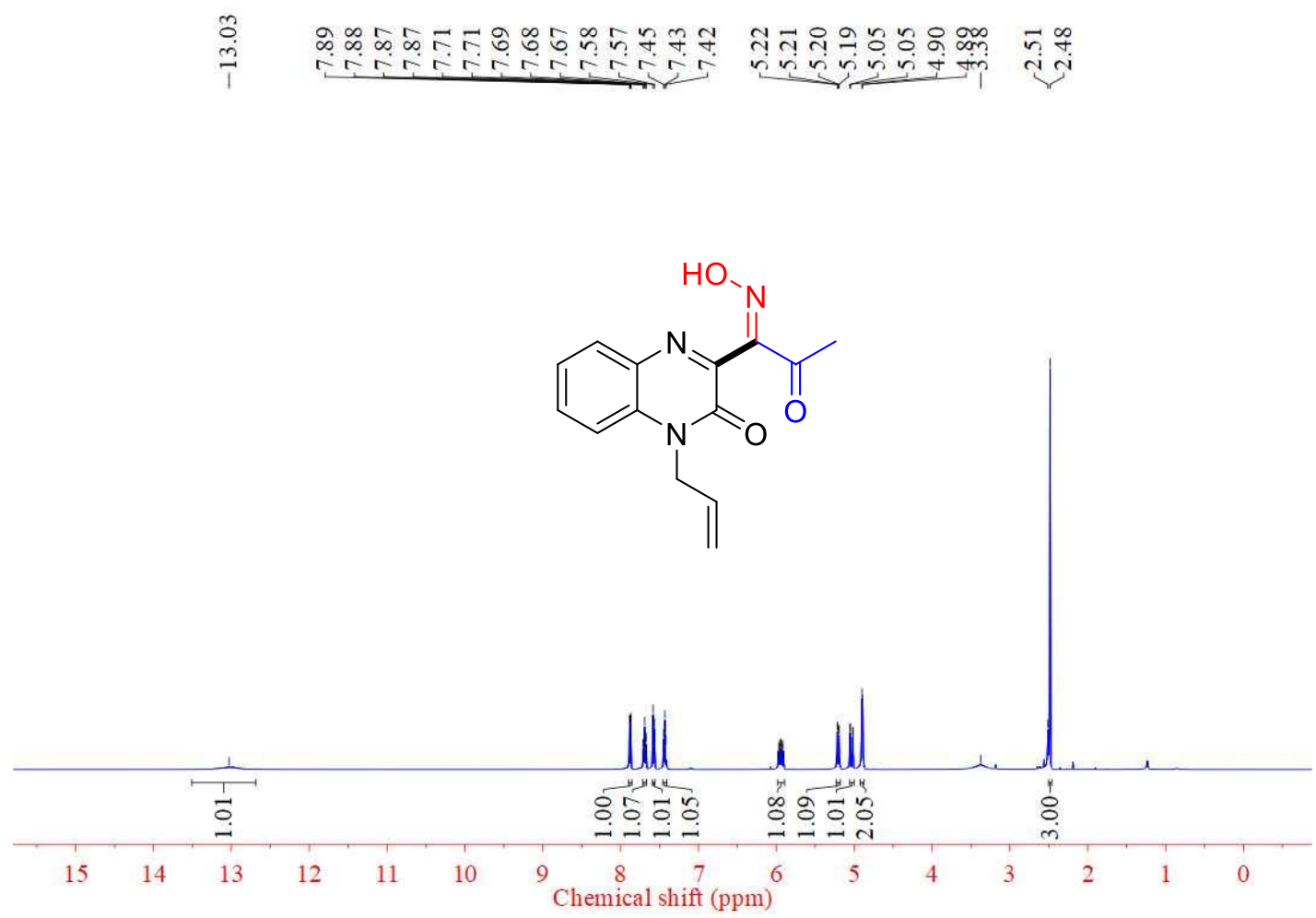

\section{$11{ }^{13} \mathrm{C}$ NMR (126 MHz, DMSO)}

\begin{tabular}{l}
$n$ \\
$\infty$ \\
2 \\
\multirow{2}{*}{}
\end{tabular}

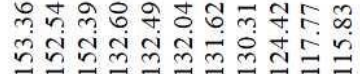

ตำ ำ 우

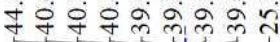<smiles>C=CCn1c(=O)c(/C(=N\O)C(C)=O)nc2ccccc21</smiles>

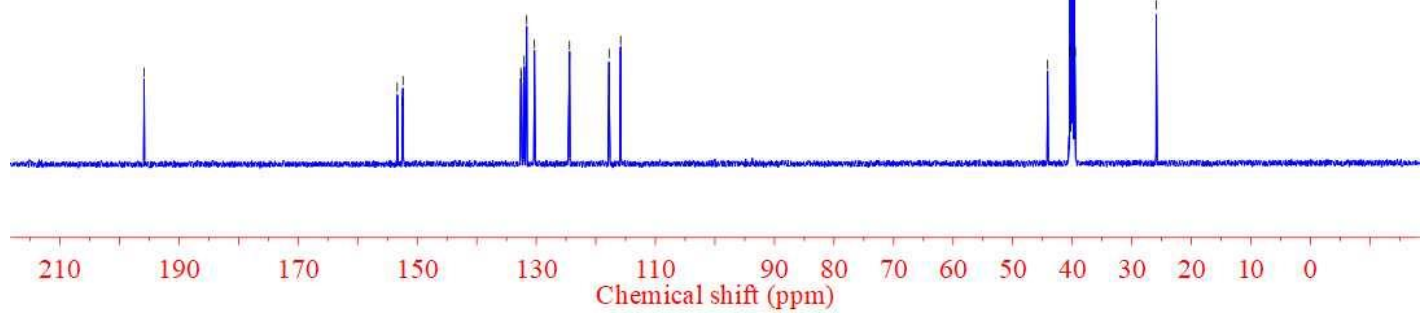




\section{$12{ }^{1} \mathrm{H}$ NMR (500 MHz, DMSO)}

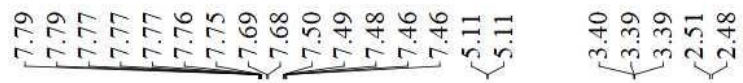<smiles>C#CCn1c(=O)c(/C(=N\O)C(C)=O)nc2ccccc21</smiles>

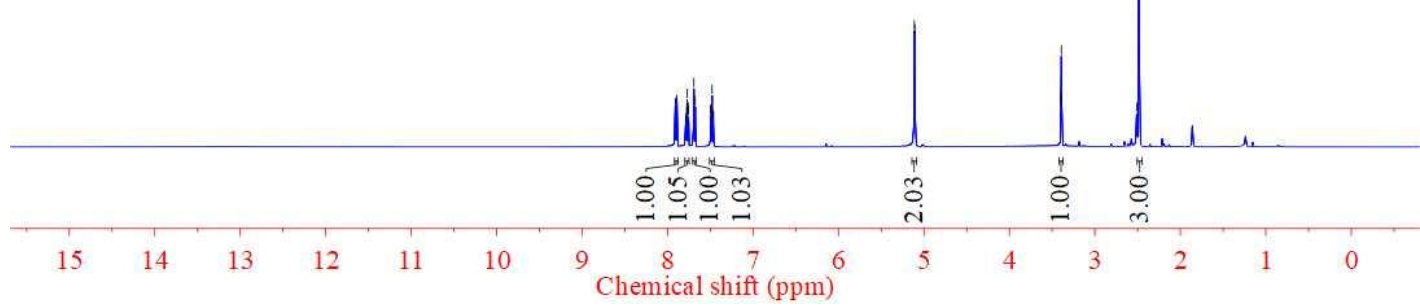

$12{ }^{13} \mathrm{C}$ NMR (126 MHz, DMSO)

ڤิْ<smiles>C#CCn1c(=O)c(/C(=N\O)C(C)=O)nc2ccccc21</smiles>

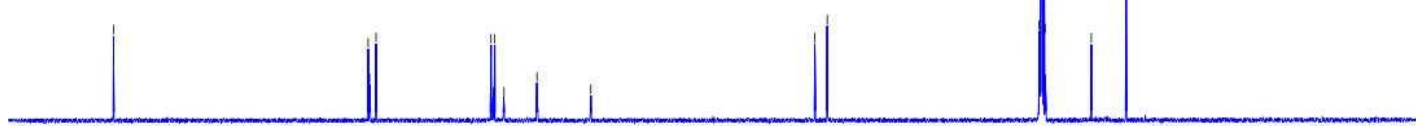

$210 \quad 190$

$170 \quad 150$

$\begin{array}{llllllllll}90 & 80 & 70 & 60 & 50 & 40 & 30 & 20 & 10 & 0\end{array}$ 


\section{$13{ }^{1}$ H NMR (500 MHz, DMSO)}
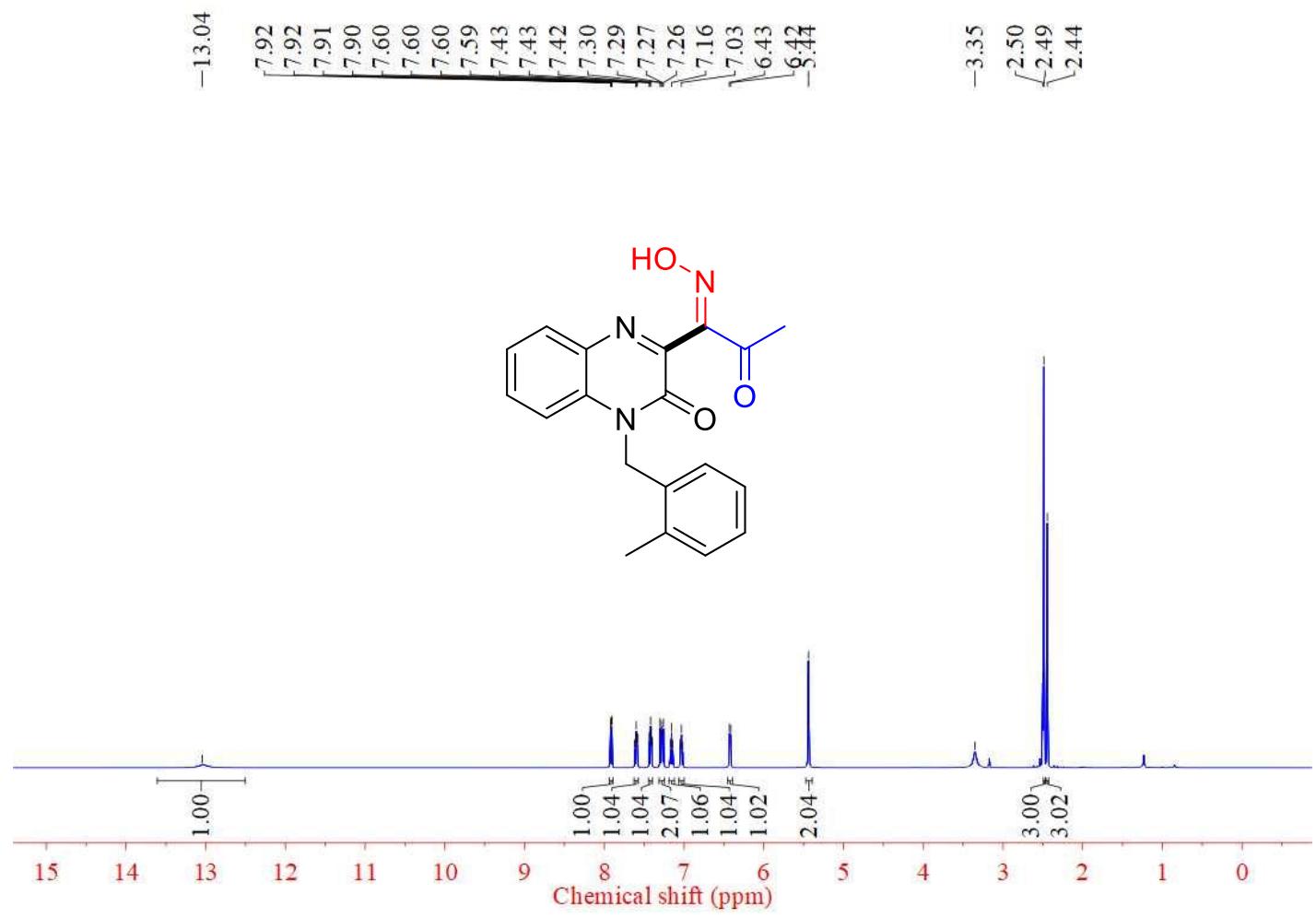

\section{$13{ }^{13} \mathrm{C}$ NMR (126 MHz, DMSO)}

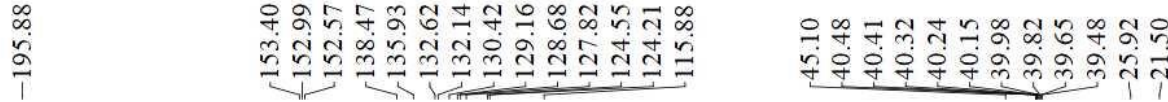

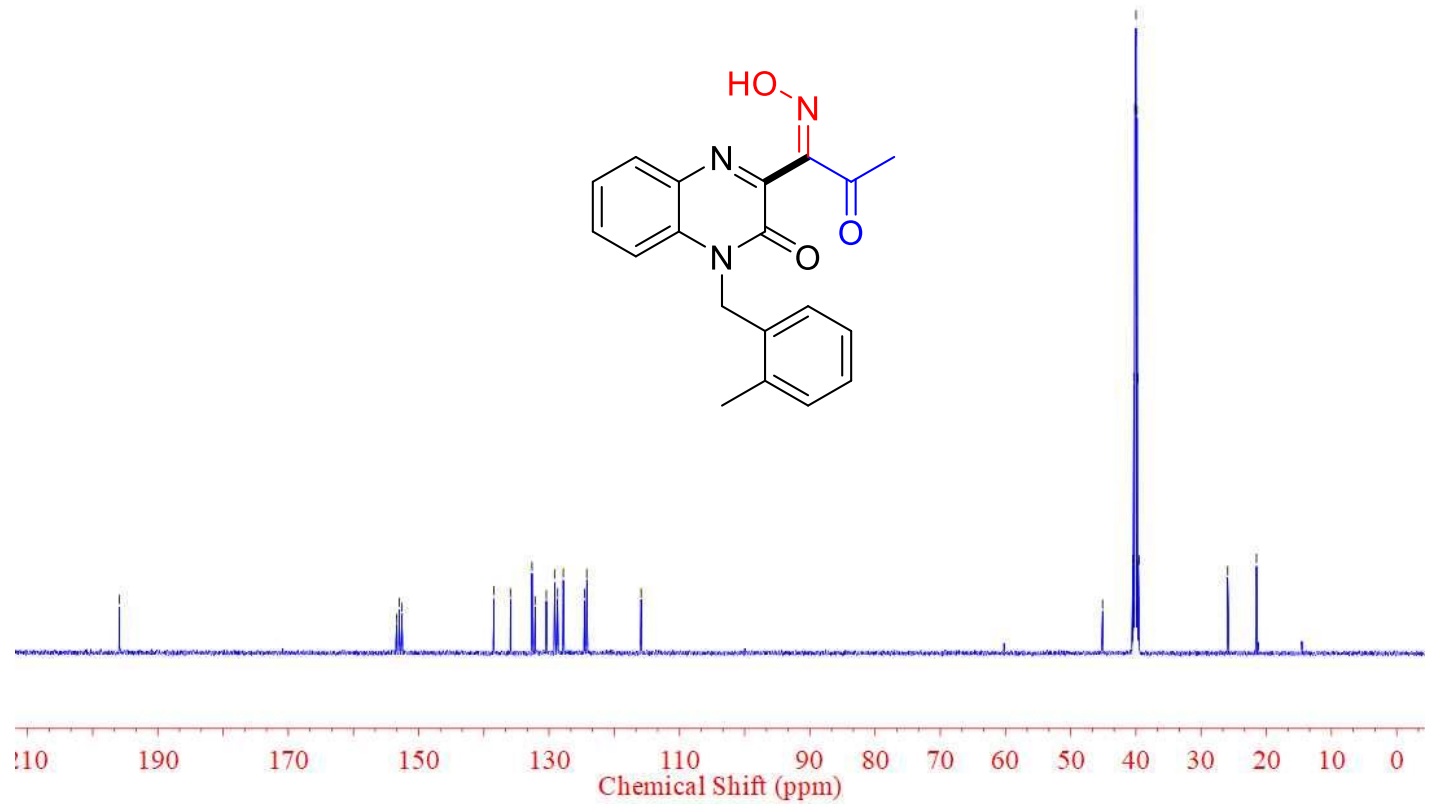




\section{$14{ }^{1} \mathrm{H}$ NMR (500 MHz, DMSO)}

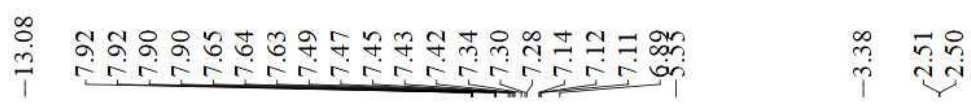

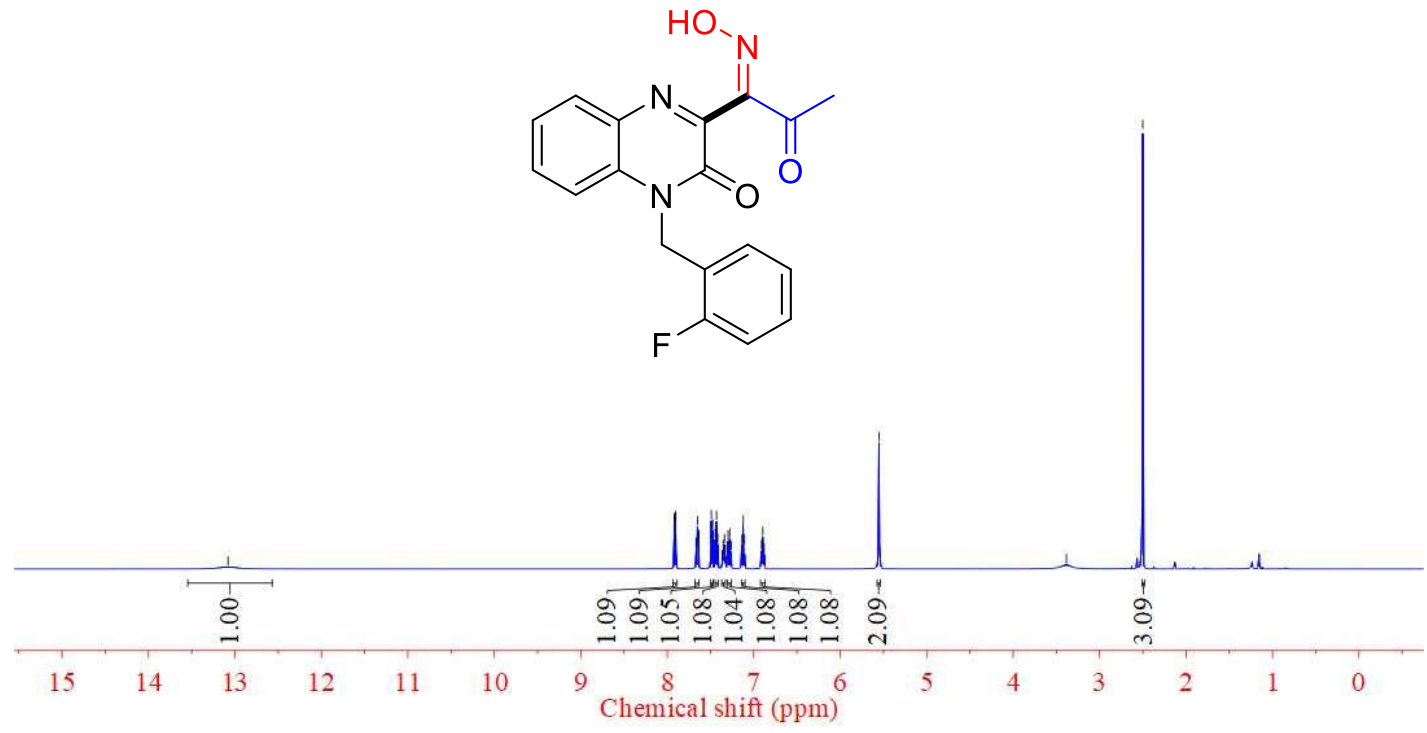

\section{$14{ }^{13} \mathrm{C}$ NMR (126 MHz, DMSO)}

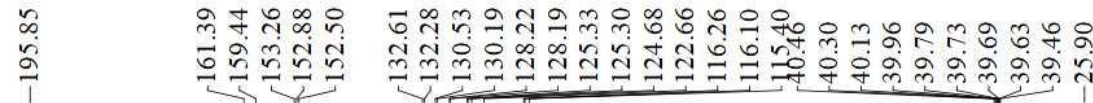

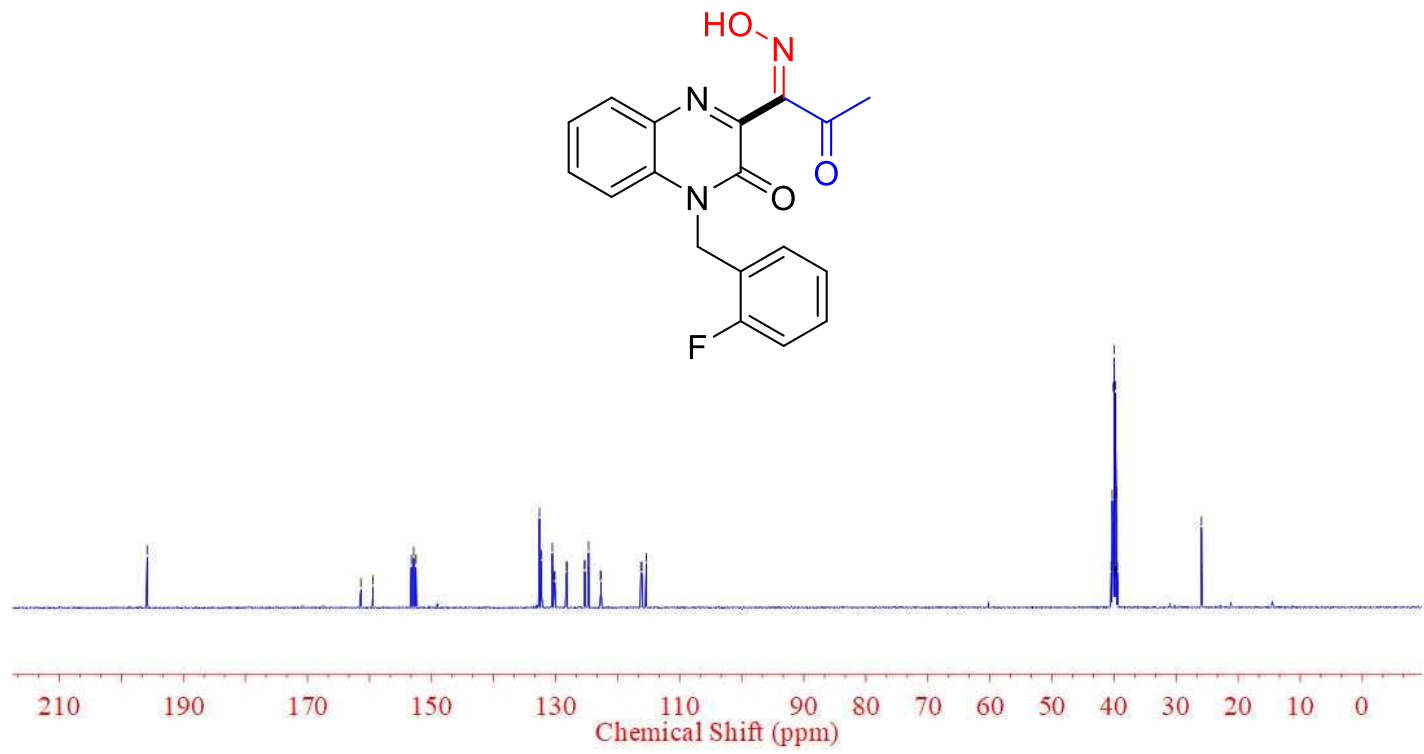




\section{$14{ }^{19}$ F NMR (471 MHz, DMSO)}

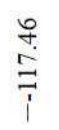

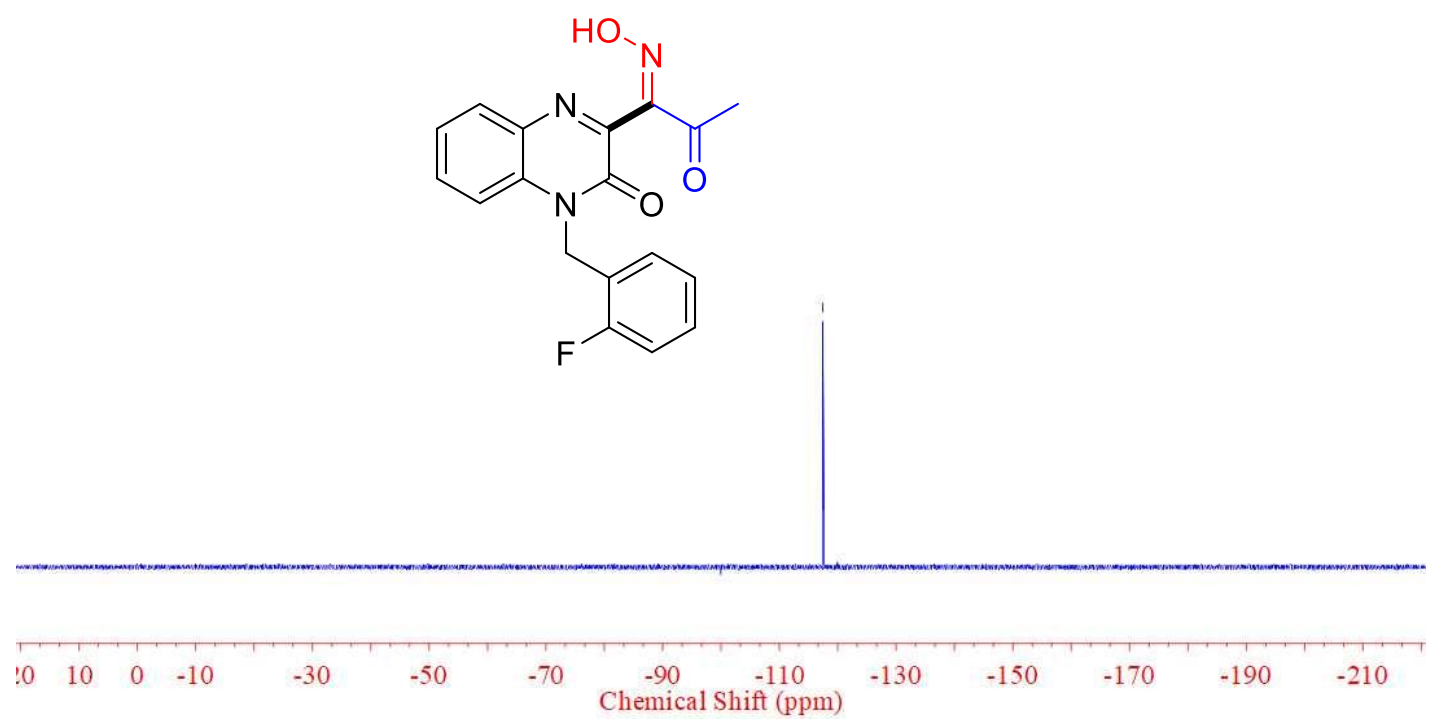

\section{$15{ }^{1}$ H NMR (500 MHz, DMSO)}

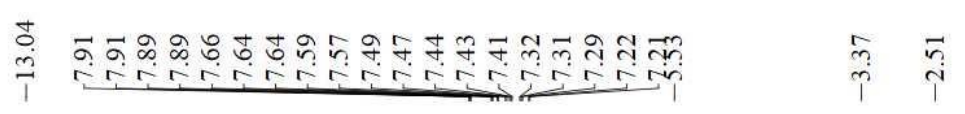

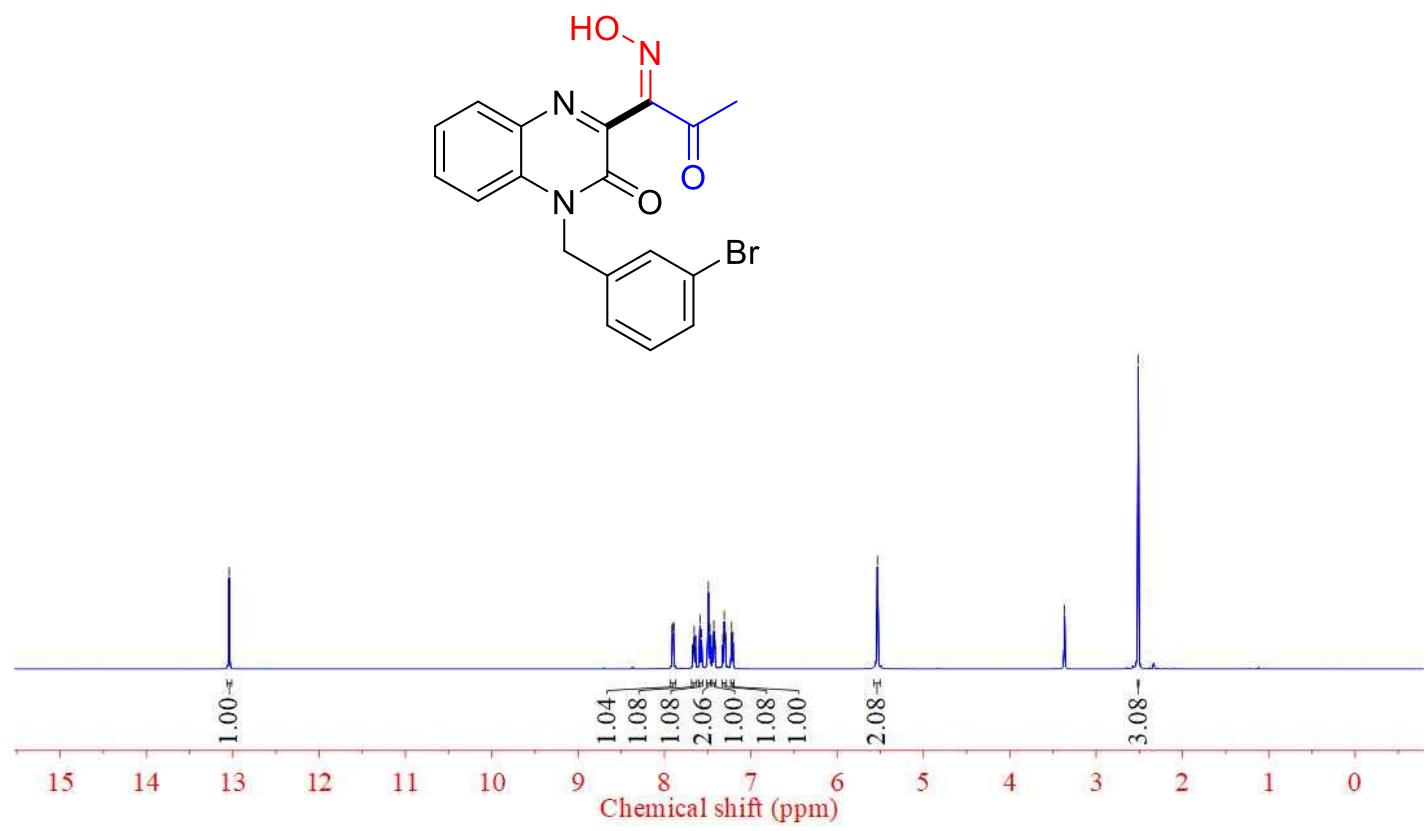




\section{$15{ }^{13} \mathrm{C}$ NMR (101 MHz, DMSO)}
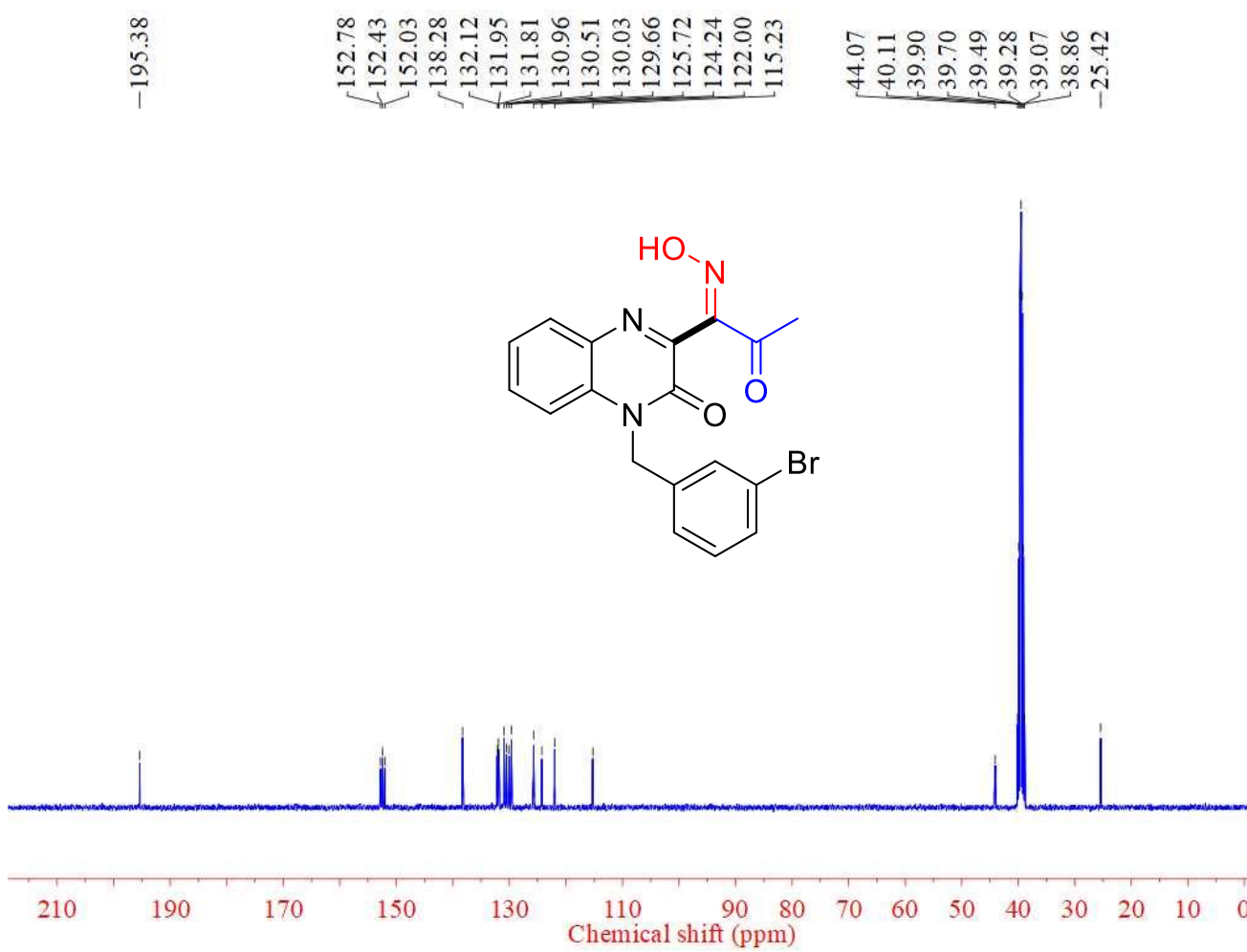

\section{$16{ }^{1} \mathrm{H}$ NMR (500 MHz, DMSO)}

彳े<smiles>CC(=O)/C(=N/O)c1nc2ccccc2n(Cc2ccc(C)cc2)c1=O</smiles>

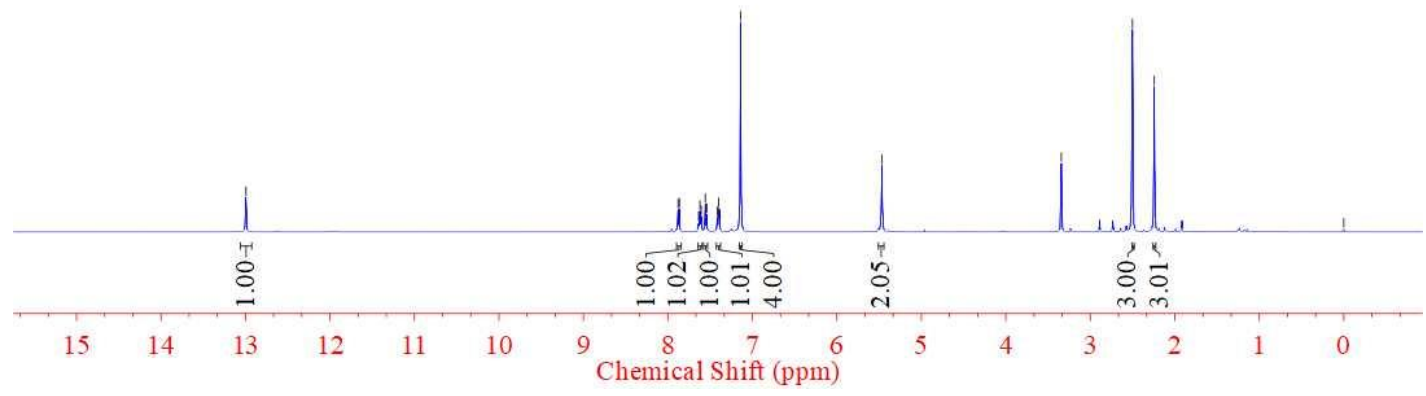




\section{$16{ }^{13} \mathrm{C}$ NMR (126 MHz, DMSO)}

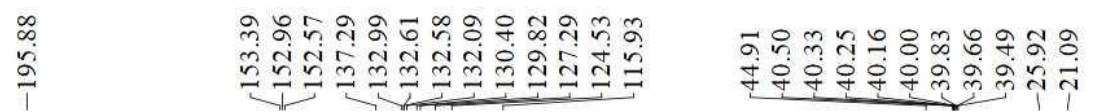<smiles>CC(=O)/C(=N/O)c1nc2ccccc2n(Cc2ccc(C)cc2)c1=O</smiles>

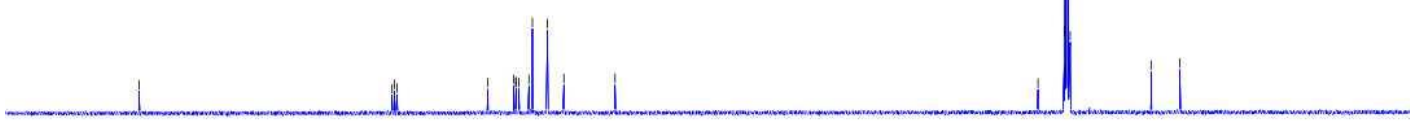

$210 \quad 190 \quad 170 \quad 150 \quad 130 \begin{gathered}110 \\ \text { Chemical Shift }(\mathrm{ppm})\end{gathered} \quad \begin{array}{llllllllll}80 & 70 & 60 & 50 & 40 & 30 & 20 & 10\end{array}$

\section{$17{ }^{1}$ H NMR (500 MHz, DMSO)}
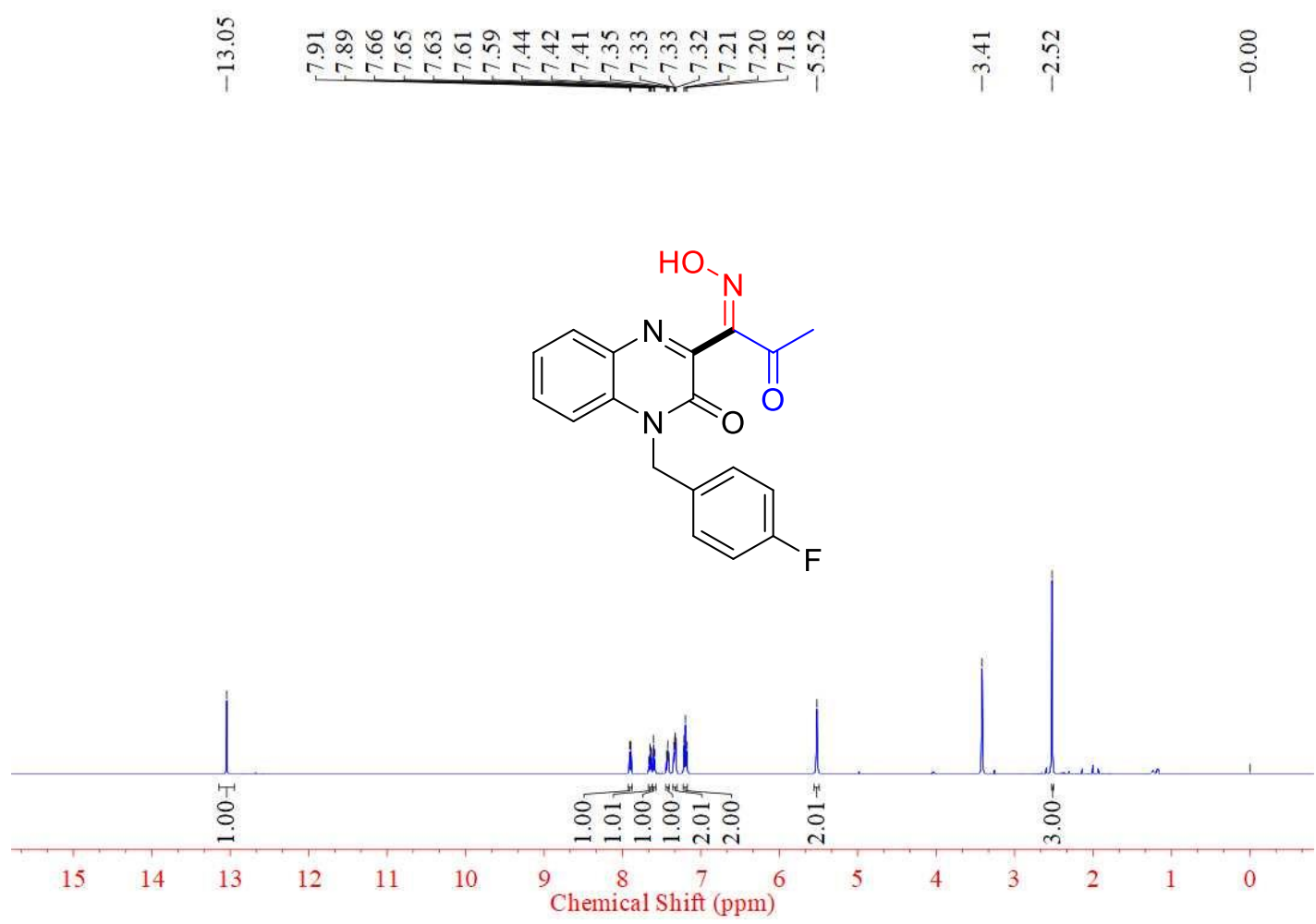


\section{$17{ }^{13} \mathrm{C}$ NMR (126 MHz, DMSO)}

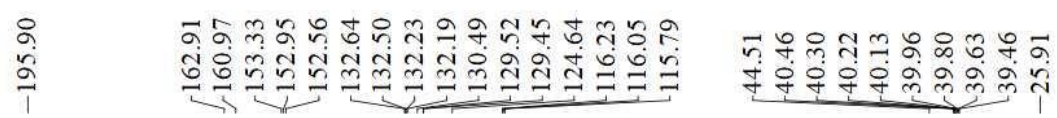<smiles>CC(=O)/C(=N/O)c1nc2ccccc2n(Cc2ccc(F)cc2)c1=O</smiles>
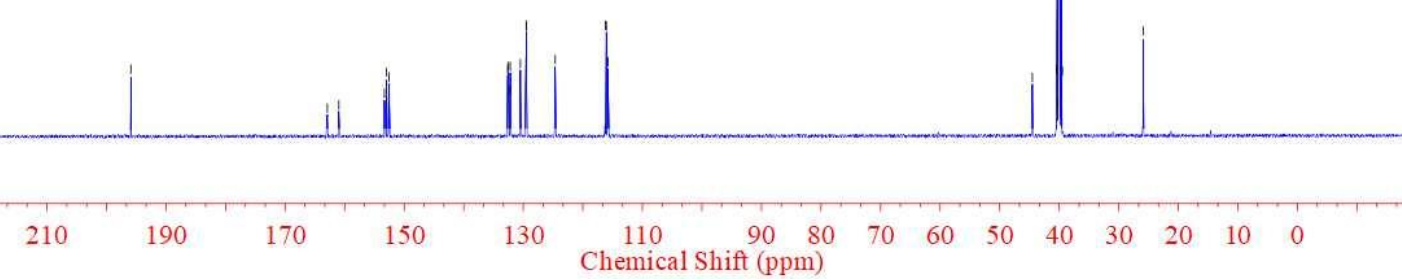

\section{$17{ }^{19}$ F NMR (471 MHz, DMSO)}

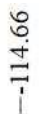<smiles>CC(=O)/C(=N/O)c1nc2ccccc2n(Cc2ccc(F)cc2)c1=O</smiles>
$\begin{array}{lllll}0 & 10 & 0 & -10\end{array}$
$-30$
$-50$
$-70$
Chemical Shift $(\mathrm{ppm})$ 


\section{$18{ }^{1}$ H NMR (500 MHz, DMSO)}
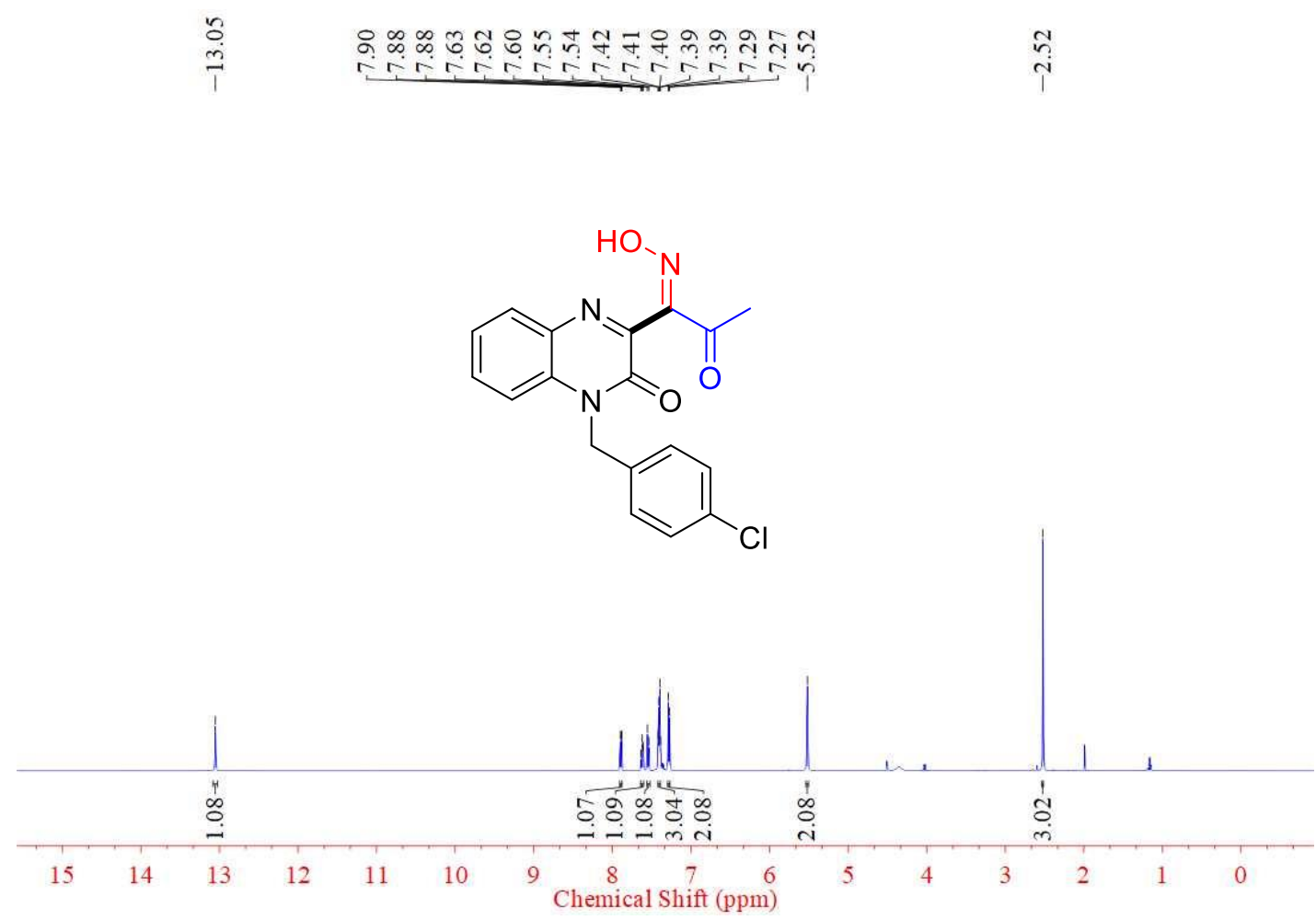

\section{$18{ }^{13} \mathrm{C}$ NMR (126 MHz, DMSO)}

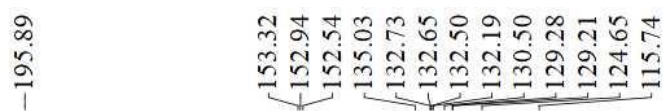

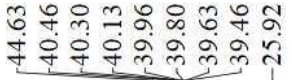<smiles>CC(=O)/C(=N/O)c1nc2ccccc2n(Cc2ccc(Cl)cc2)c1=O</smiles>

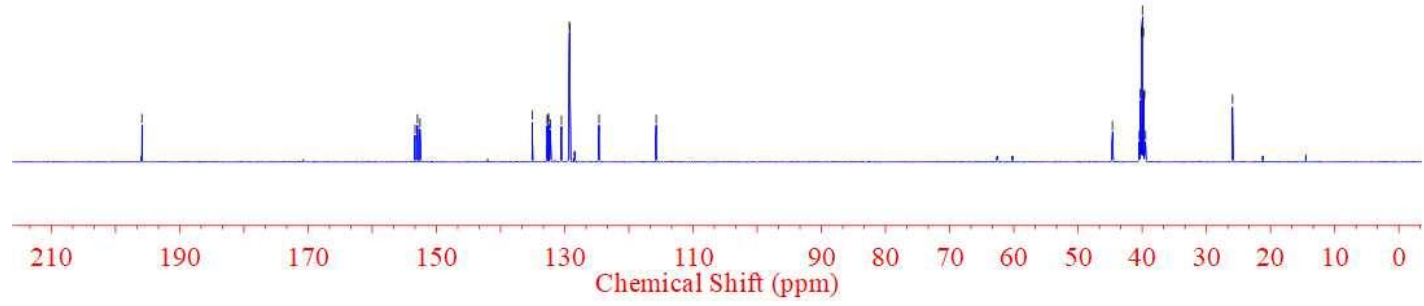


$19{ }^{1}$ H NMR (500 MHz, DMSO)

\begin{tabular}{ll}
$\infty$ \\
\hdashline
\end{tabular}<smiles>CC(=O)/C(=N/O)c1nc2c(C)cccc2n(C)c1=O</smiles>

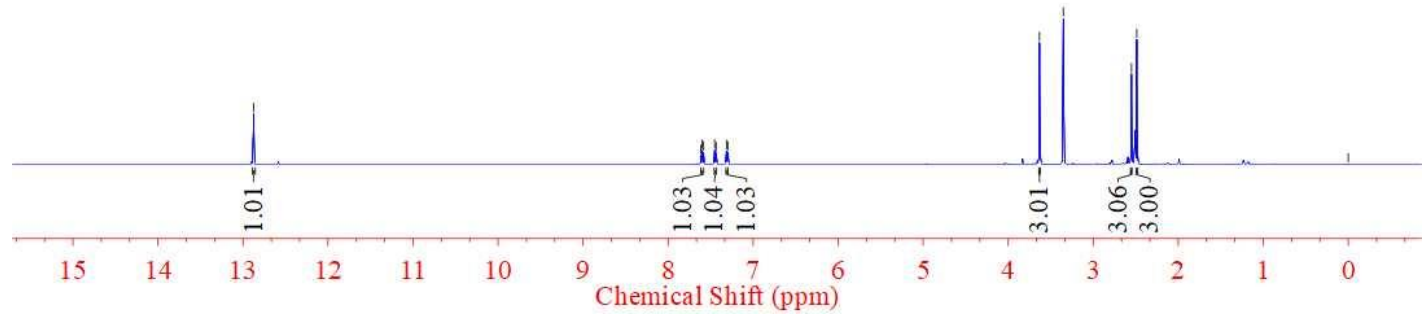

$19{ }^{13} \mathrm{C}$ NMR (126 MHz, DMSO)

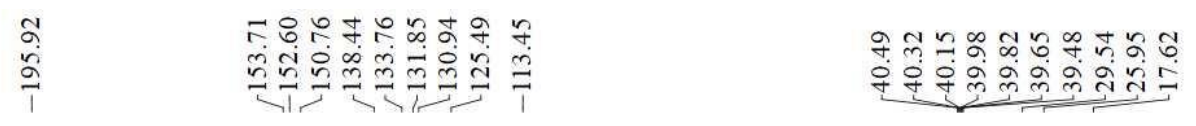<smiles>CC(=O)/C(=N/O)c1nc2c(C)cccc2n(C)c1=O</smiles>

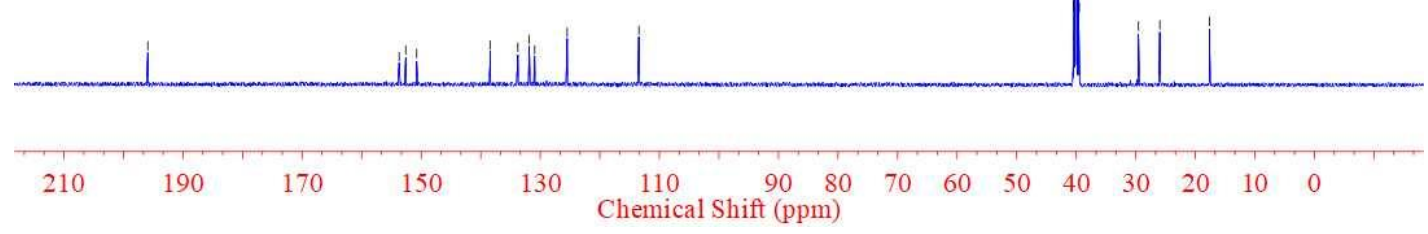




\section{$20{ }^{1}$ H NMR (500 MHz, DMSO)}

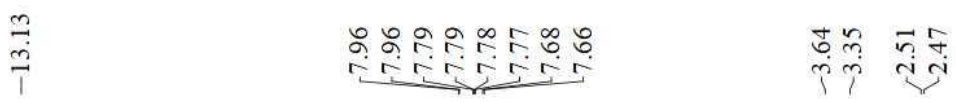

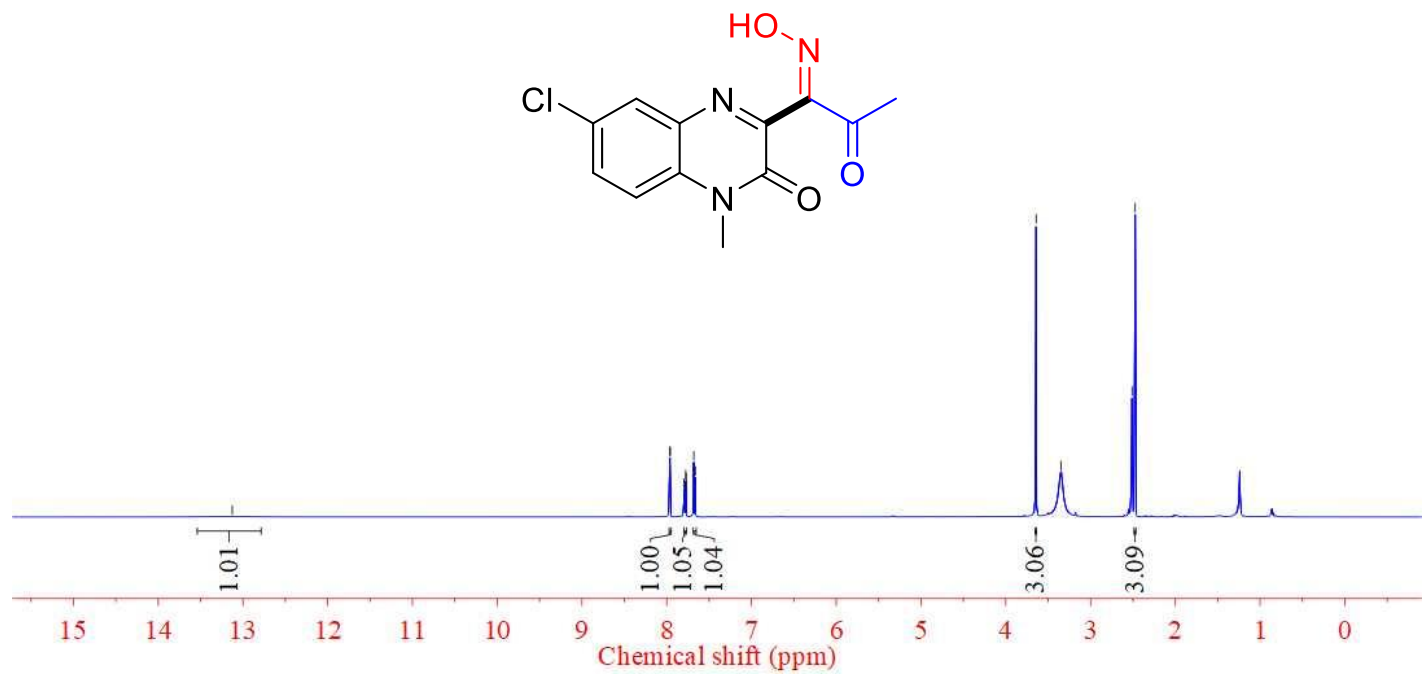

\section{$20{ }^{13} \mathrm{C}$ NMR (126 MHz, DMSO)}
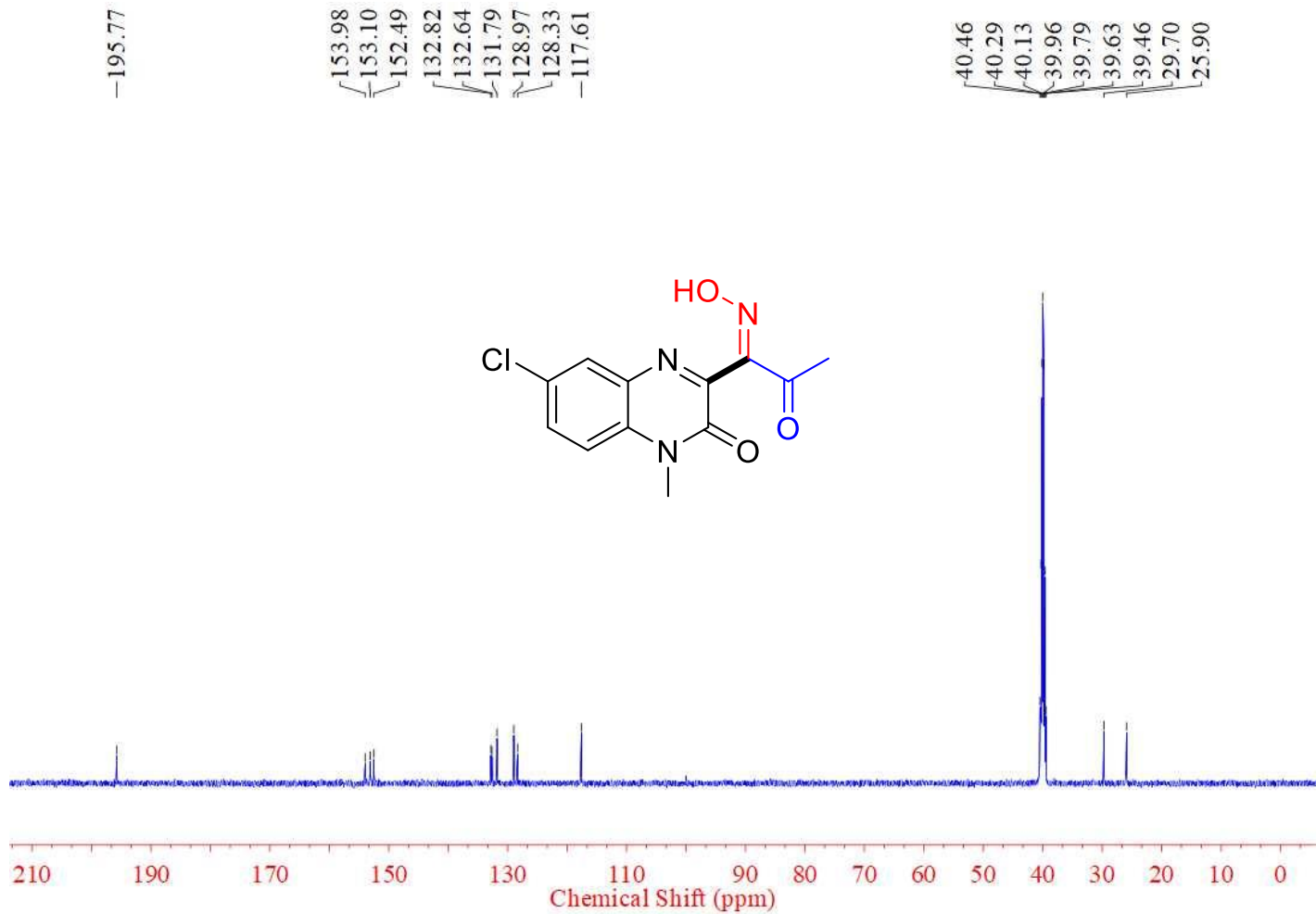


\section{$21{ }^{1}$ H NMR (500 MHz, DMSO)}
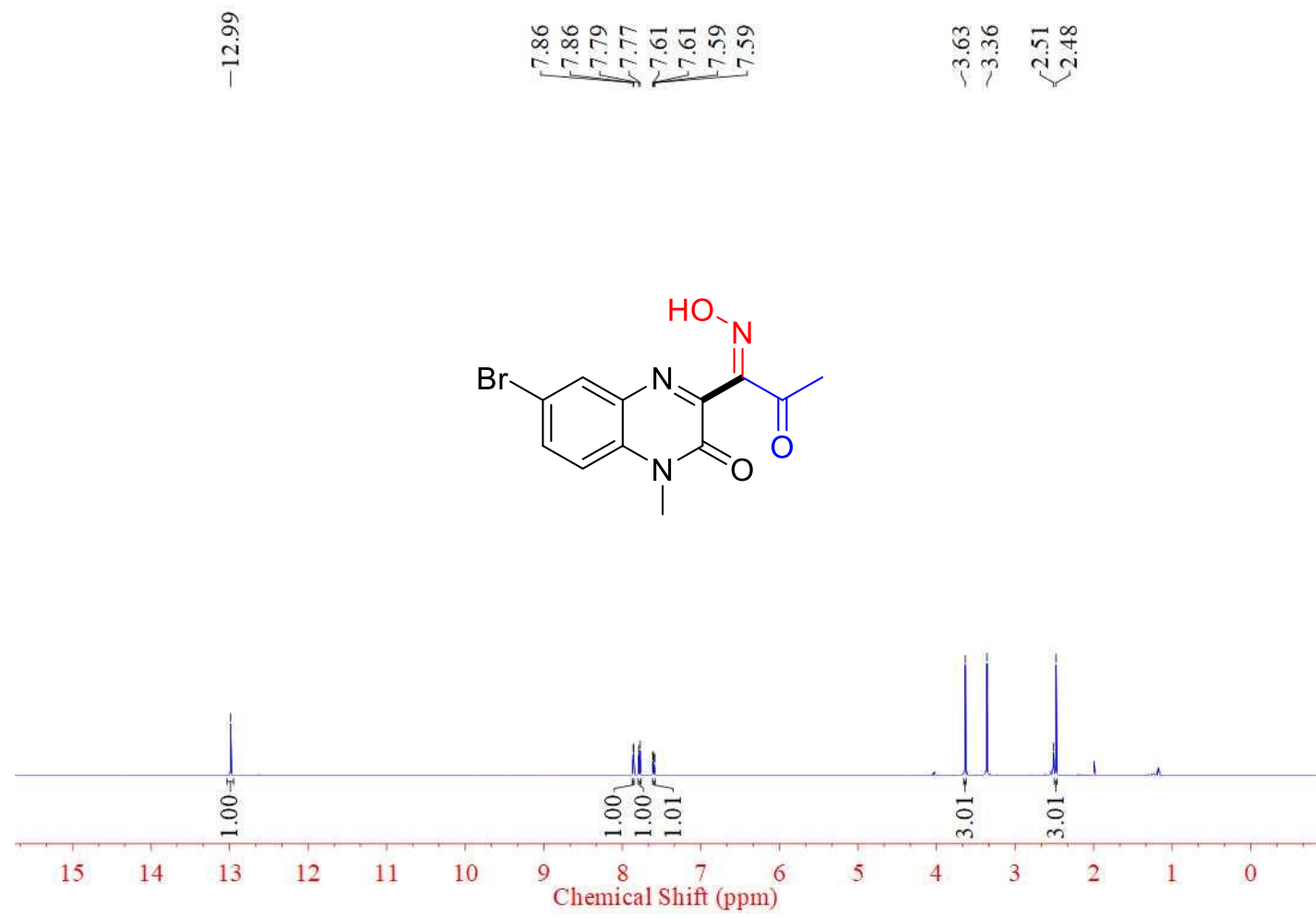

\section{$21{ }^{13} \mathrm{C}$ NMR (126 MHz, DMSO)}

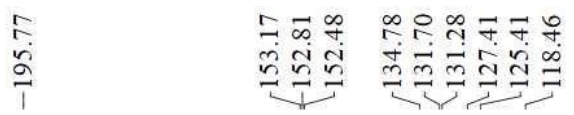<smiles>CC(=O)/C(=N/O)c1nc2cc(Br)ccc2n(C)c1=O</smiles>

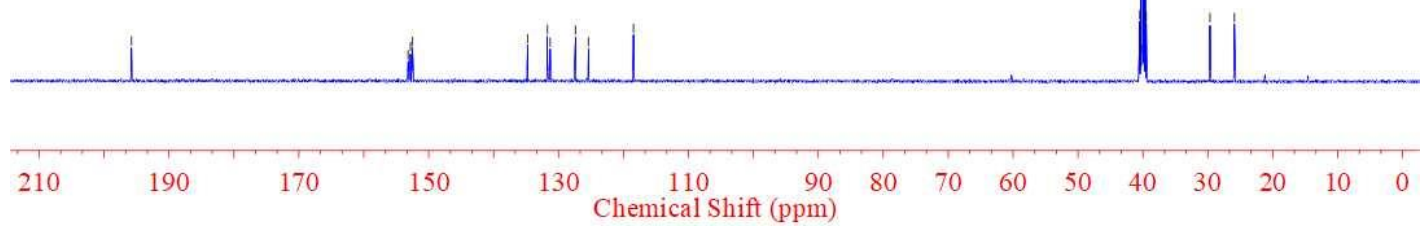




\section{$22{ }^{1}$ H NMR (500 MHz, DMSO)}

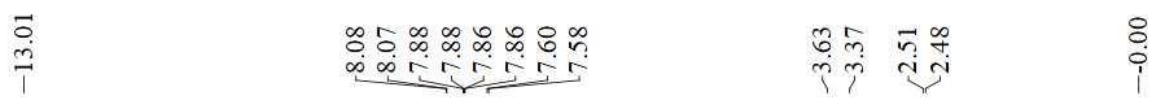<smiles>CC(=O)/C(=N/O)c1nc2ccc(Br)cc2n(C)c1=O</smiles>

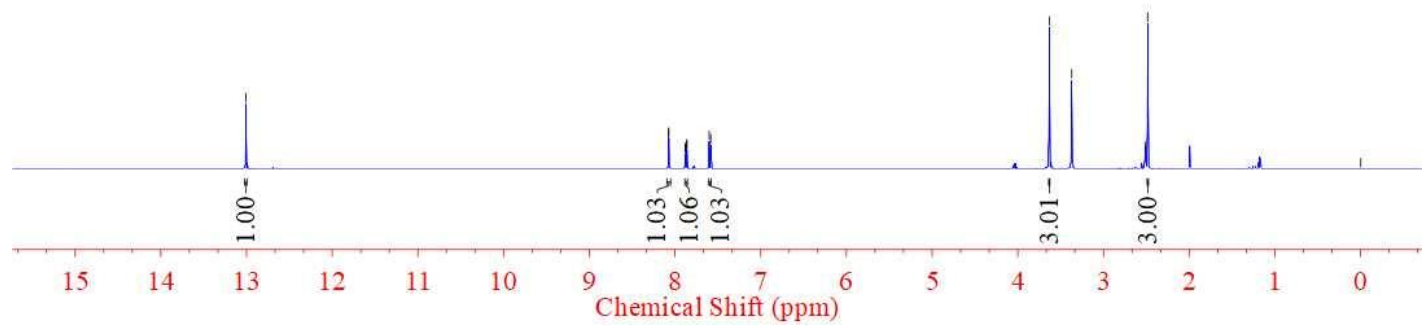

\section{$22{ }^{13} \mathrm{C}$ NMR (126 MHz, DMSO)}
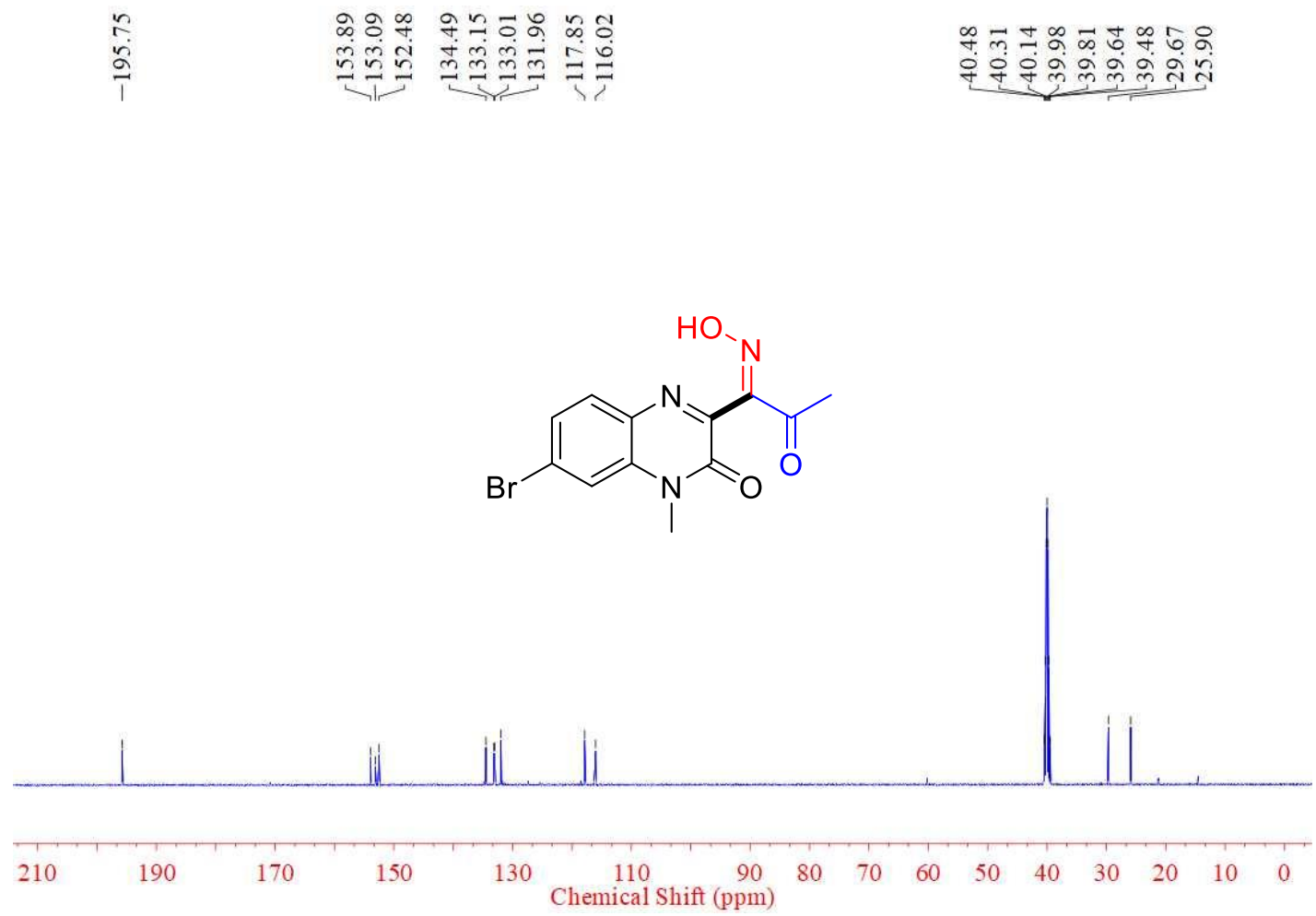


\section{$23{ }^{1}$ H NMR (500 MHz, DMSO)}

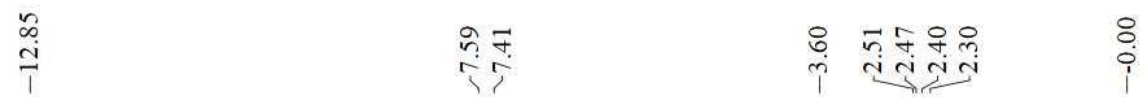<smiles>CC(=O)/C(=N/O)c1nc2cc(C)c(C)cc2n(C)c1=O</smiles>

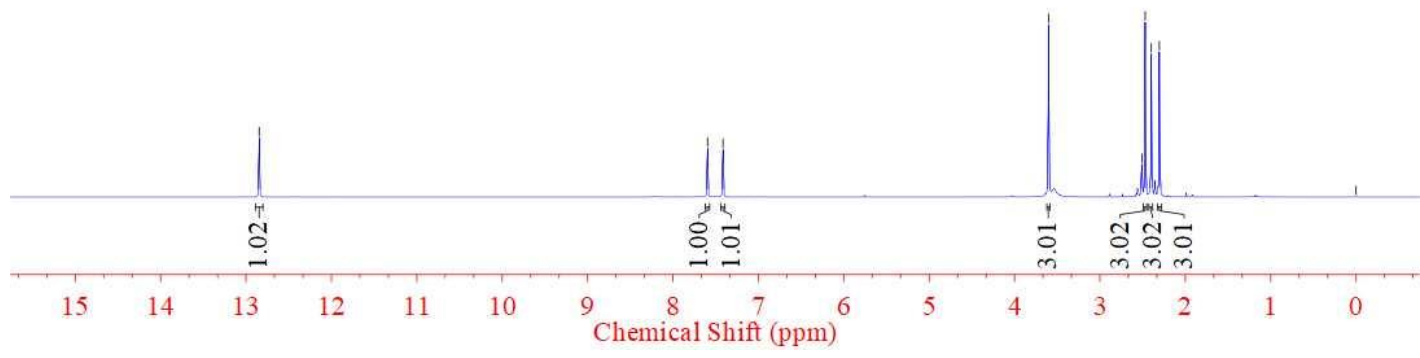

\section{$23{ }^{13} \mathrm{C}$ NMR (126 MHz, DMSO)}
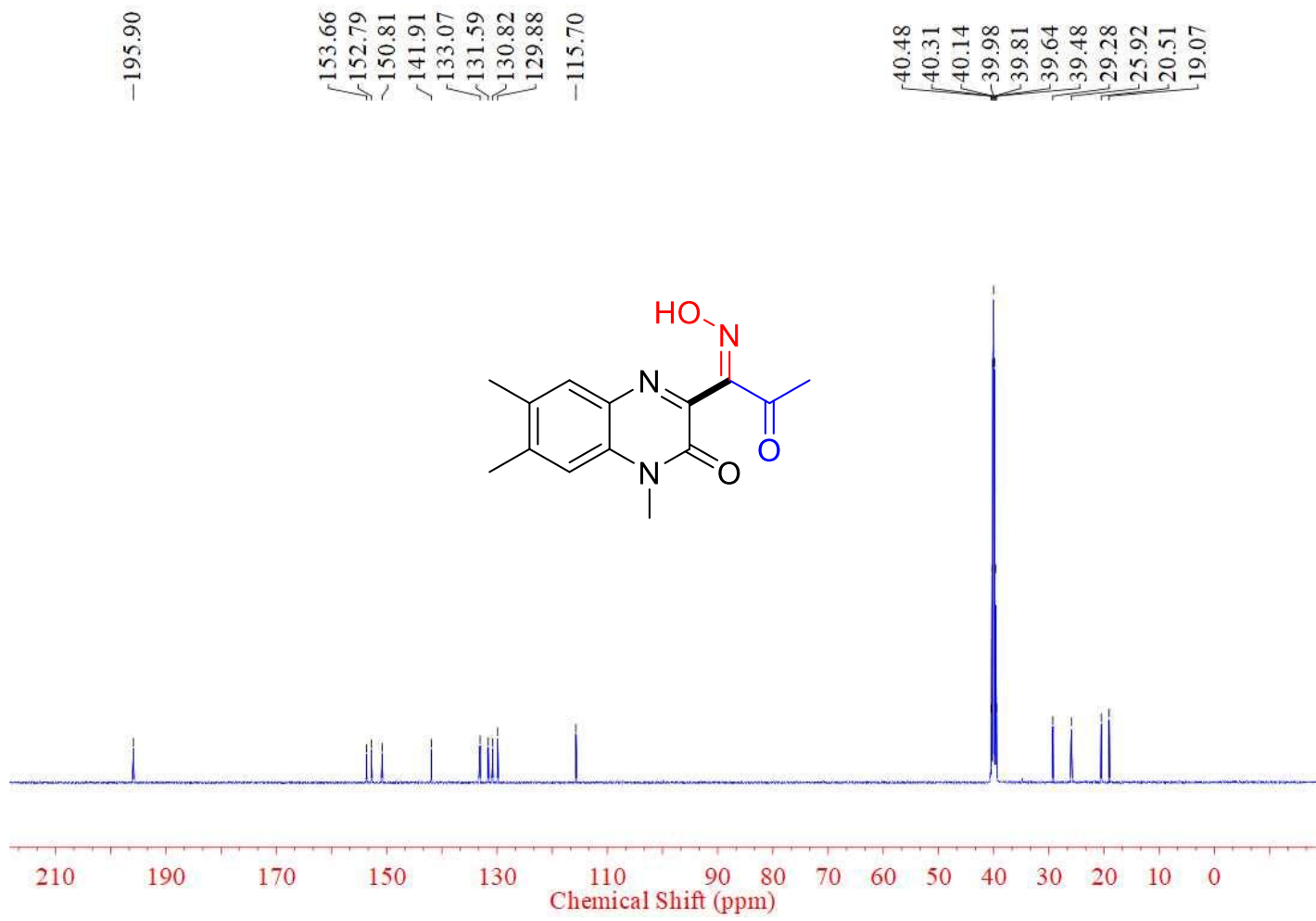


\section{$24{ }^{1}$ H NMR (500 MHz, DMSO)}

\section{㩆要}

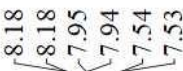

in

$\stackrel{8}{\circ}$<smiles>CC(=O)/C(=N/O)c1nc2cc(C(F)(F)F)ccc2[nH]c1=O</smiles>

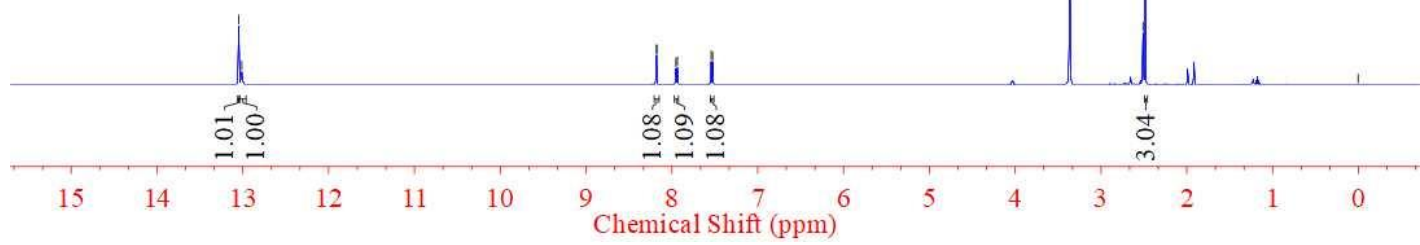

\section{$24{ }^{13} \mathrm{C}$ NMR (126 MHz, DMSO)}<smiles>CC(=O)/C(=N/O)c1nc2cc(C(F)(F)F)ccc2[nH]c1=O</smiles>

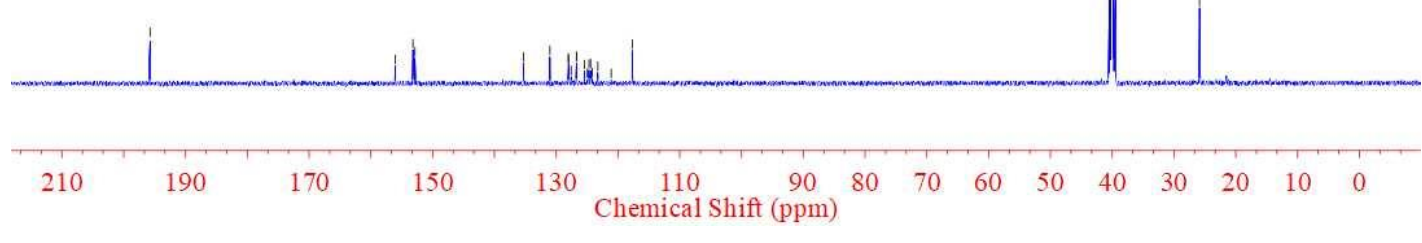




\section{$24{ }^{19}$ F NMR (471 MHz, DMSO)}

$n$
$\vdots$
0
$\vdots$<smiles>CC(=O)/C(=N/O)c1nc2cc(C(F)(F)F)ccc2[nH]c1=O</smiles>

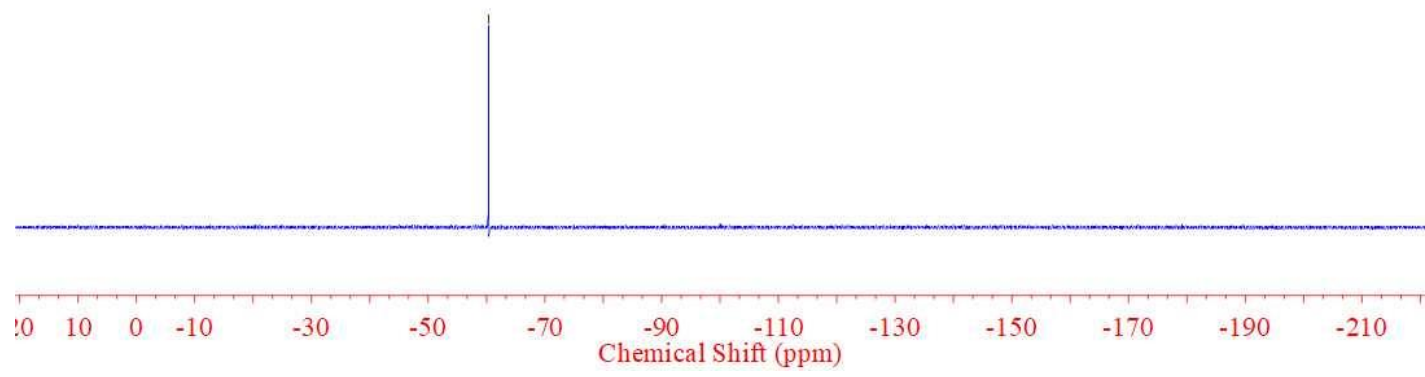

\section{$25{ }^{1}$ H NMR (500 MHz, DMSO)}

$$
\text { जे }
$$

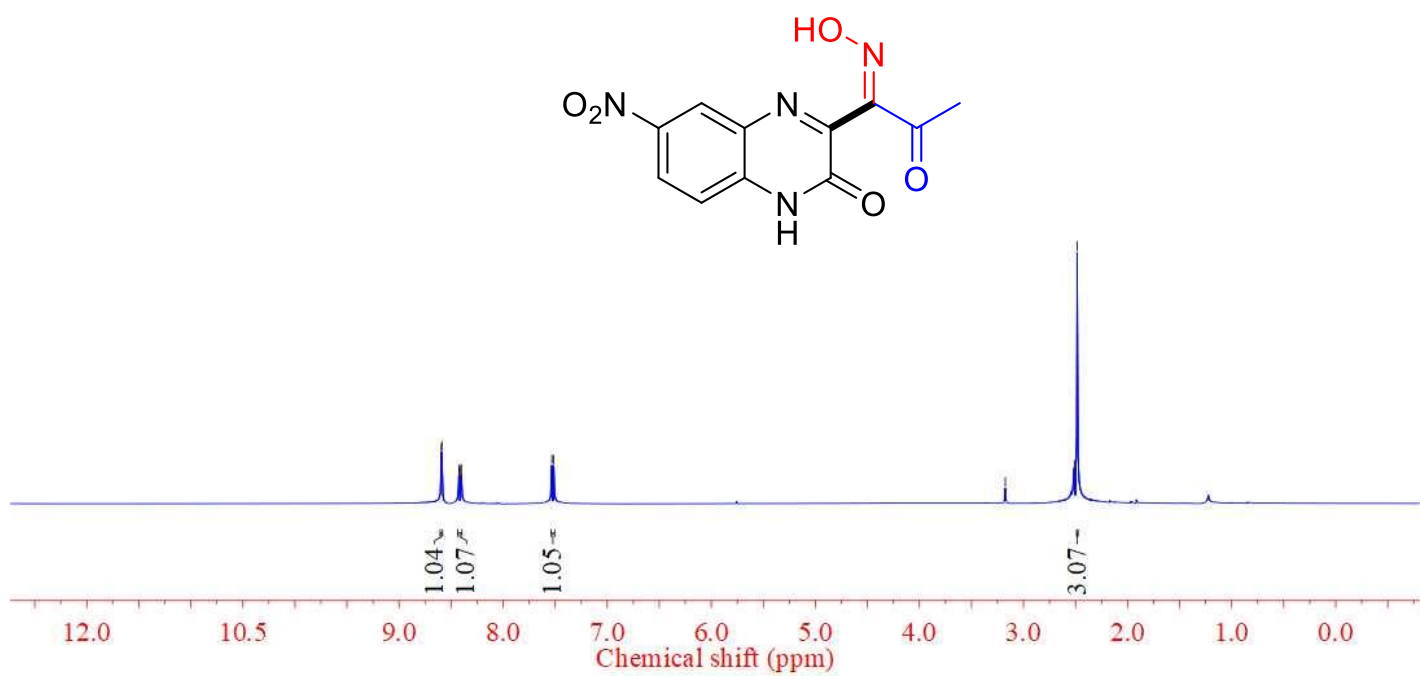


$25{ }^{13} \mathrm{C}$ NMR (126 MHz, DMSO)

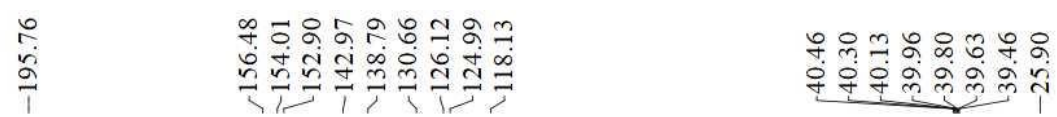<smiles>CC(=O)/C(=N/O)c1nc2cc([N+](=O)[O-])ccc2[nH]c1=O</smiles>

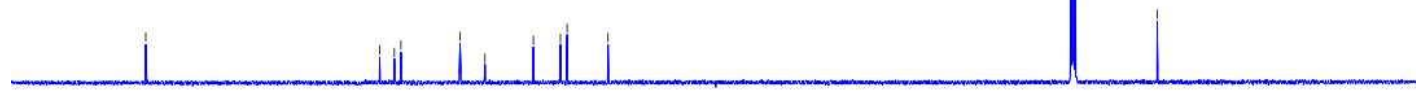

$210 \quad 190 \quad 170 \quad 150 \quad 130 \quad \begin{gathered}110 \\ \text { Chemical shift }(\mathrm{ppm})\end{gathered}$

\section{$26{ }^{1}$ H NMR (500 MHz, DMSO)}

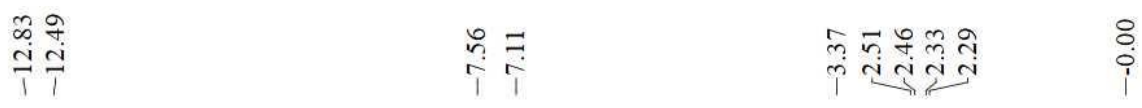<smiles>CC(=O)/C(=N/O)c1nc2cc(C)c(C)cc2[nH]c1=O</smiles>

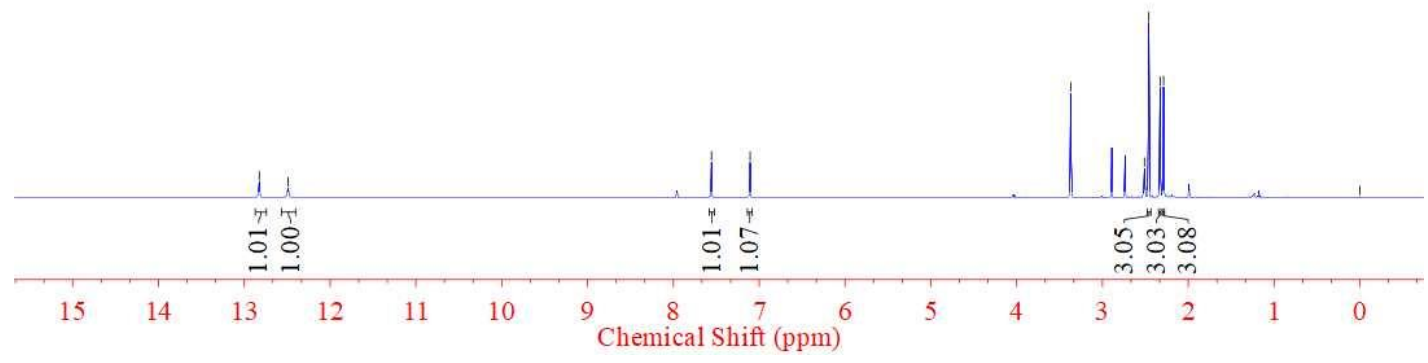




\section{$26{ }^{13} \mathrm{C}$ NMR (126 MHz, DMSO)}
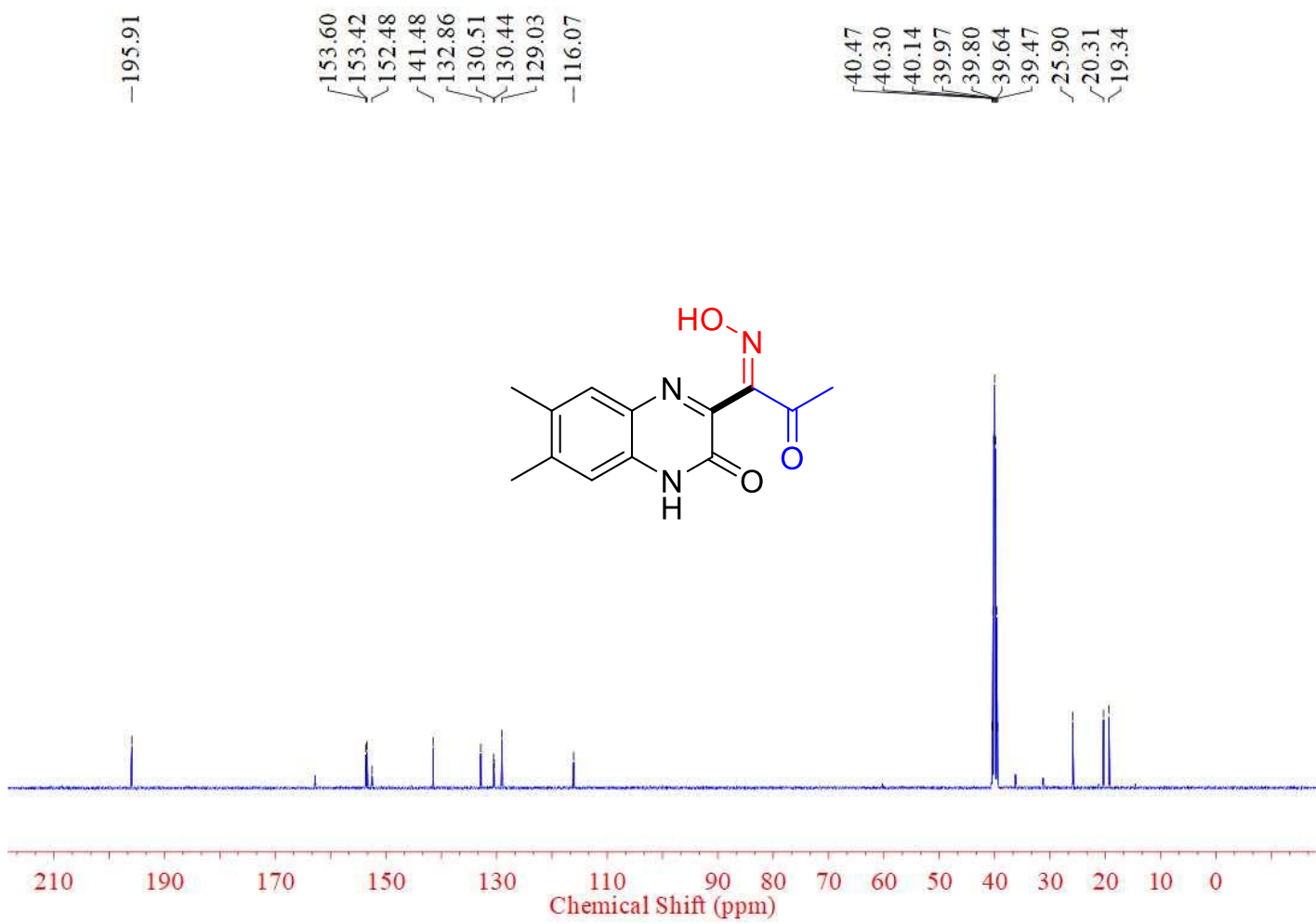

\section{$27{ }^{1}$ H NMR (500 MHz, DMSO)}

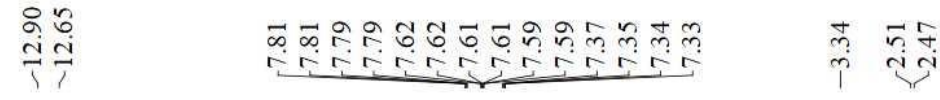

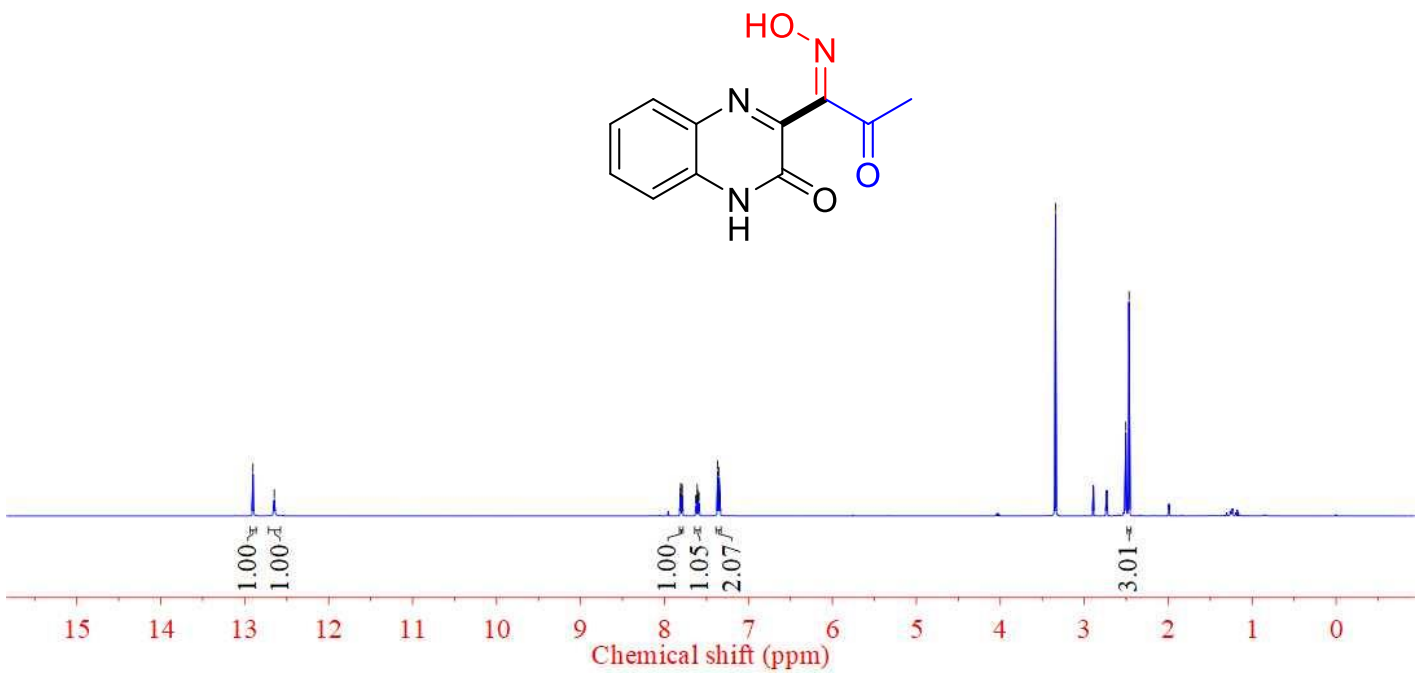


$27{ }^{13} \mathrm{C}$ NMR (126 MHz, DMSO)

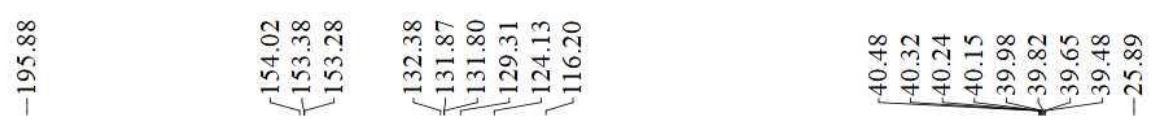<smiles>CC(=O)/C(=N/O)c1nc2ccccc2[nH]c1=O</smiles>

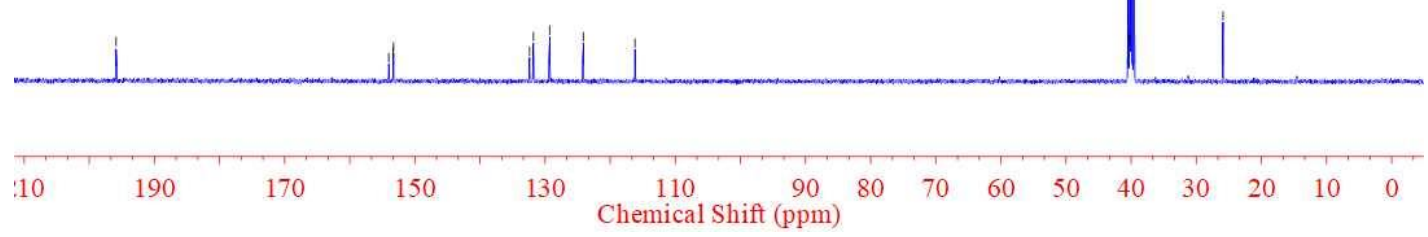

\section{$28{ }^{1}$ H NMR (500 MHz, DMSO)}

年

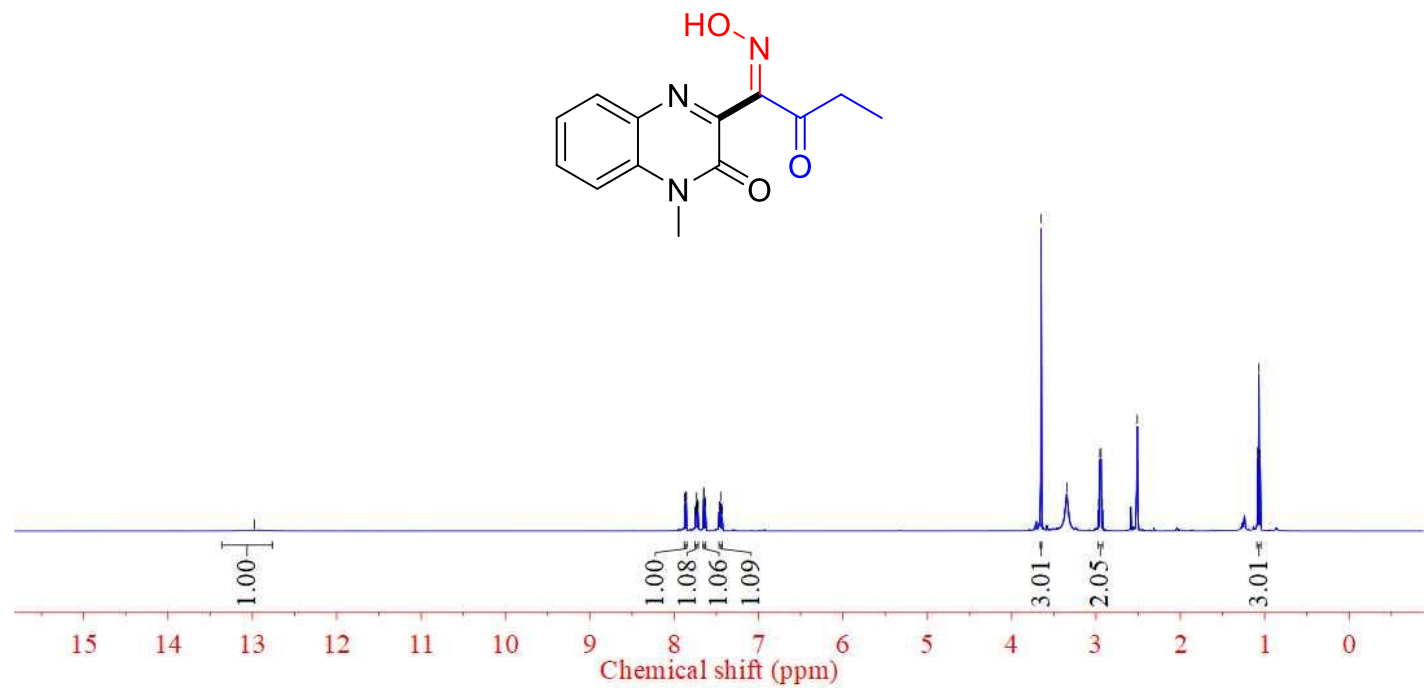


$28{ }^{13} \mathrm{C}$ NMR (126 MHz, DMSO)

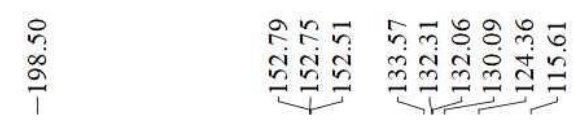

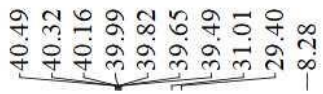<smiles>CCC(=O)/C(=N/O)c1nc2ccccc2n(C)c1=O</smiles>

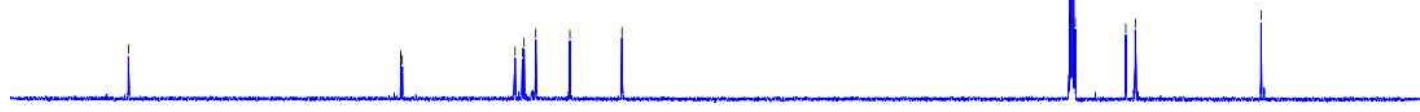

$210 \quad 190 \quad 170 \quad 150 \quad 130 \quad \begin{gathered}110 \\ \text { Chemical shift }(\mathrm{ppm})\end{gathered}$

\section{$29{ }^{1}$ H NMR (500 MHz, DMSO)}

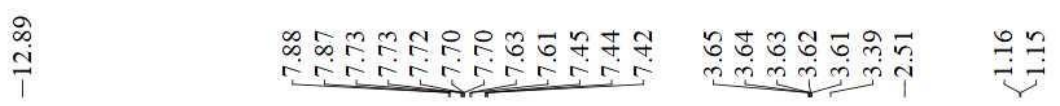<smiles>CC(C)C(=O)/C(=N\O)c1nc2ccccc2n(C)c1=O</smiles>

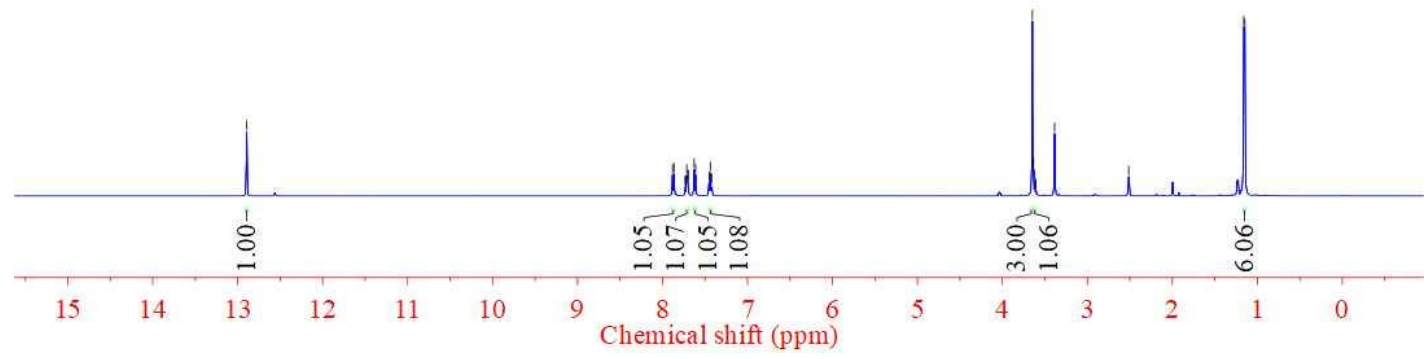


$29{ }^{13} \mathrm{C}$ NMR (126 MHz, DMSO)

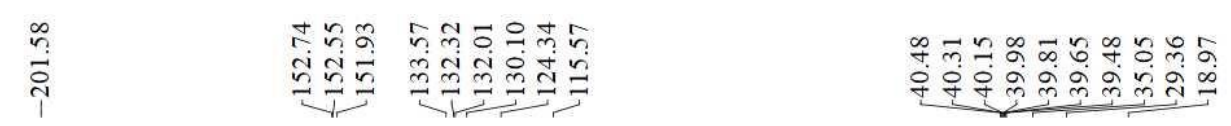<smiles>CC(C)C(=O)/C(=N/O)c1nc2ccccc2n(C)c1=O</smiles>

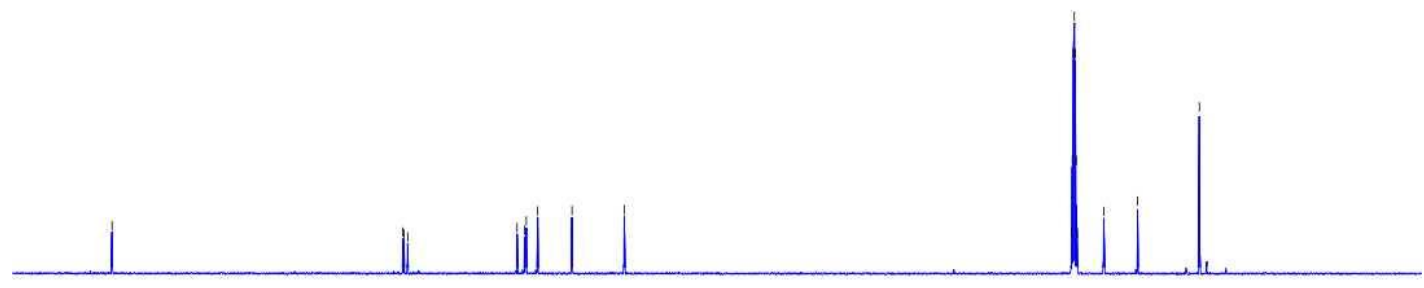

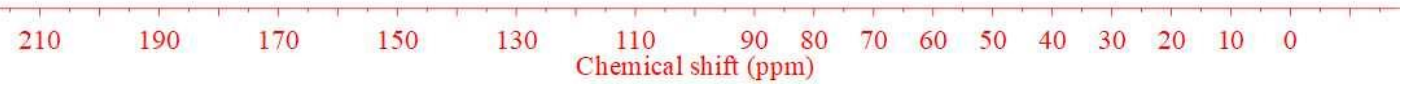

\section{$30{ }^{1}$ H NMR (500 MHz, DMSO)}

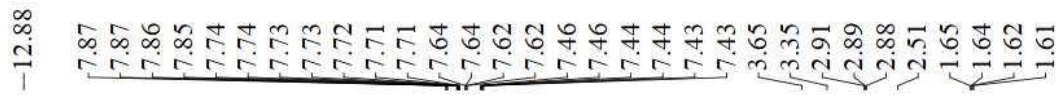

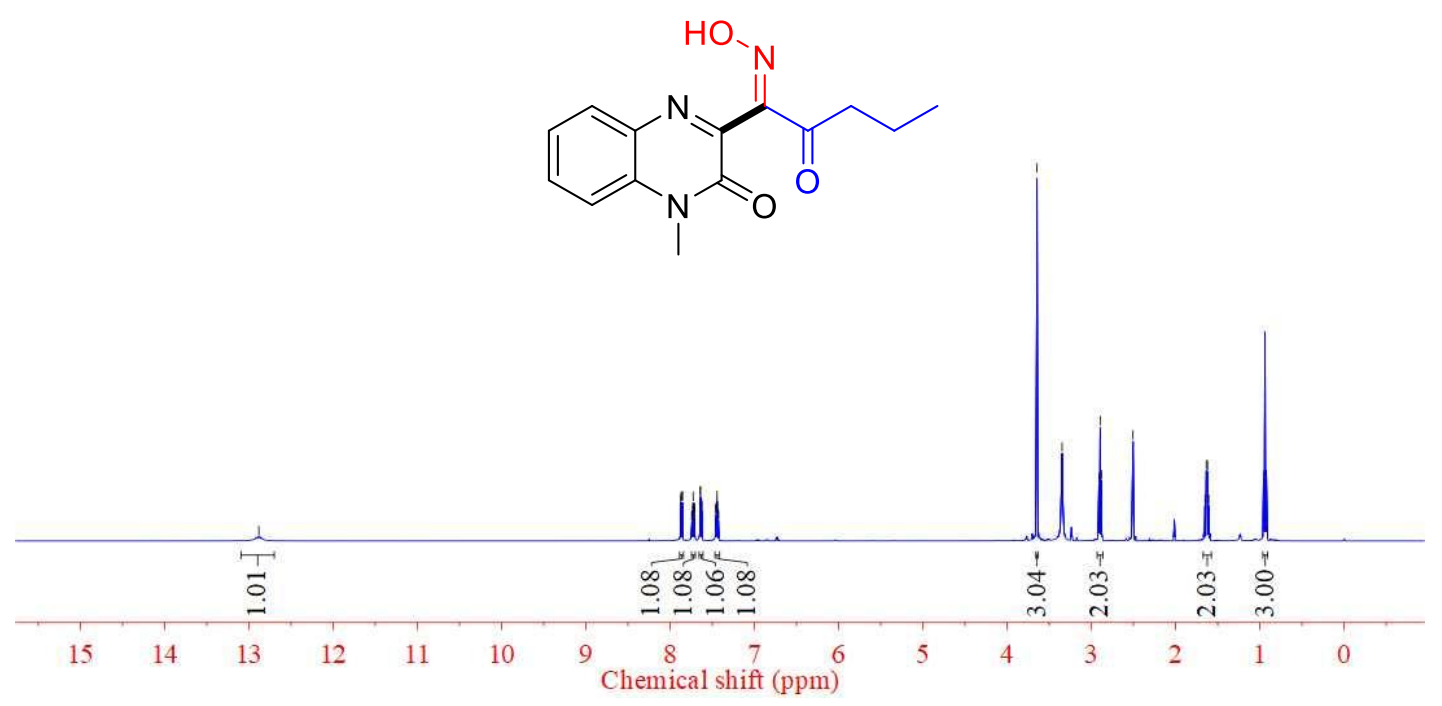


$30{ }^{13} \mathrm{C}$ NMR (126 MHz, DMSO)

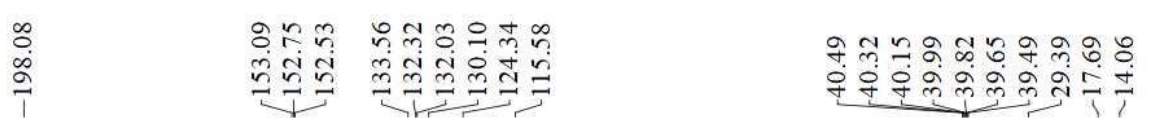

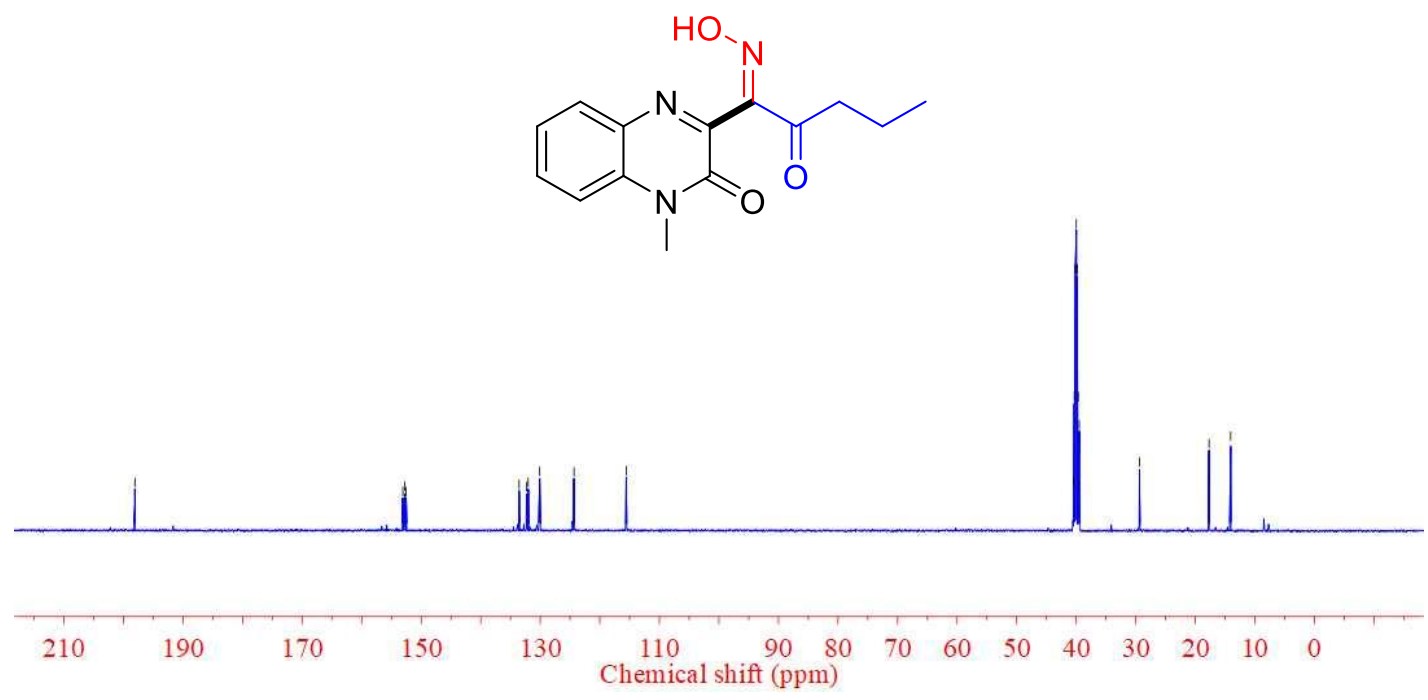

\section{$31{ }^{1}$ H NMR (500 MHz, DMSO)}

1
lom

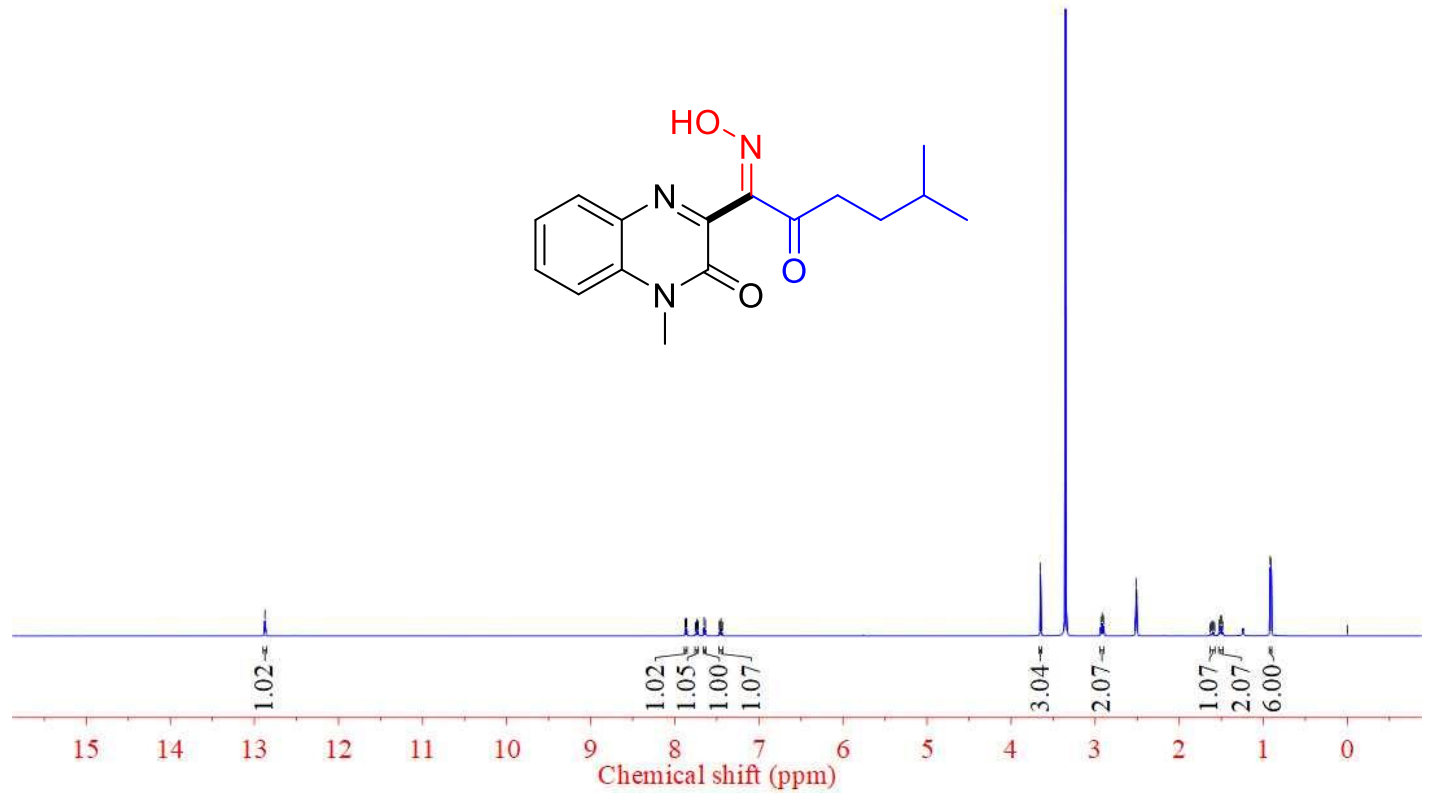


$31{ }^{13} \mathrm{C}$ NMR (126 MHz, DMSO)

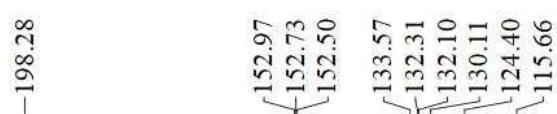

궁혀의

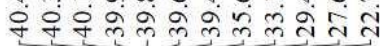<smiles>CC(C)CCC(=O)/C(=N/O)c1nc2ccccc2n(C)c1=O</smiles>

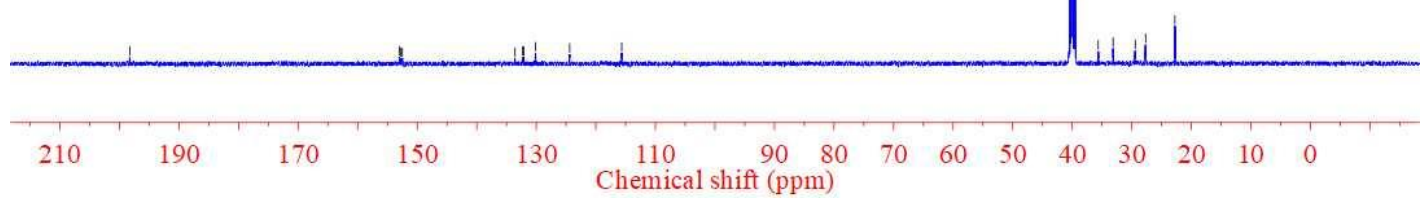

\section{$32{ }^{1} \mathrm{H}$ NMR (500 MHz, DMSO)}
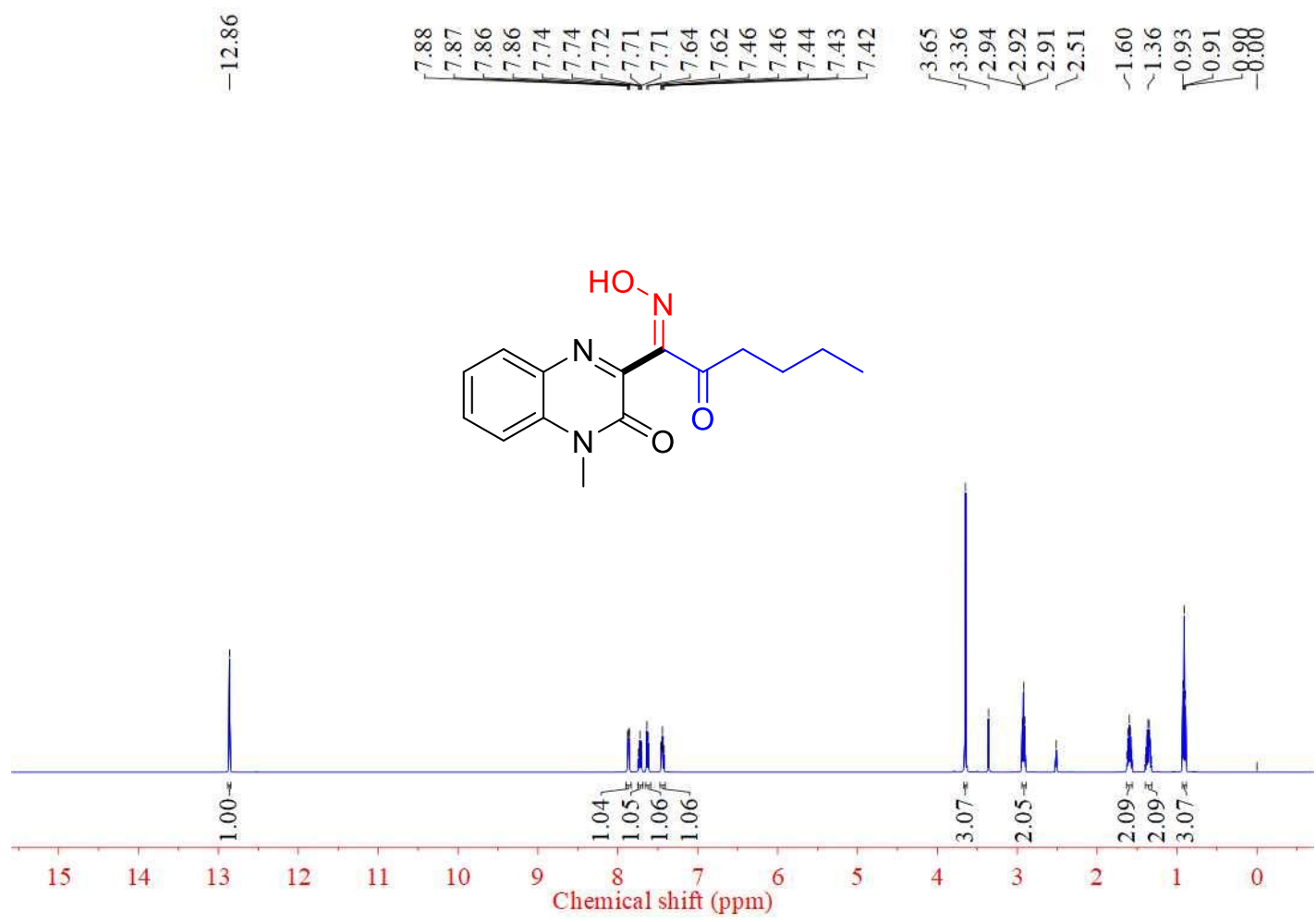
$32{ }^{13} \mathrm{C}$ NMR (101 MHz, DMSO)

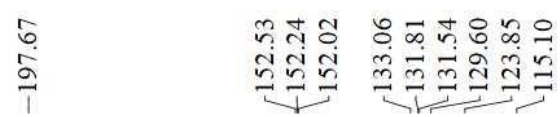

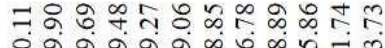

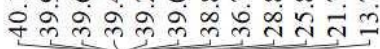<smiles>CCCCC(=O)/C(=N/O)c1nc2ccccc2n(C)c1=O</smiles>

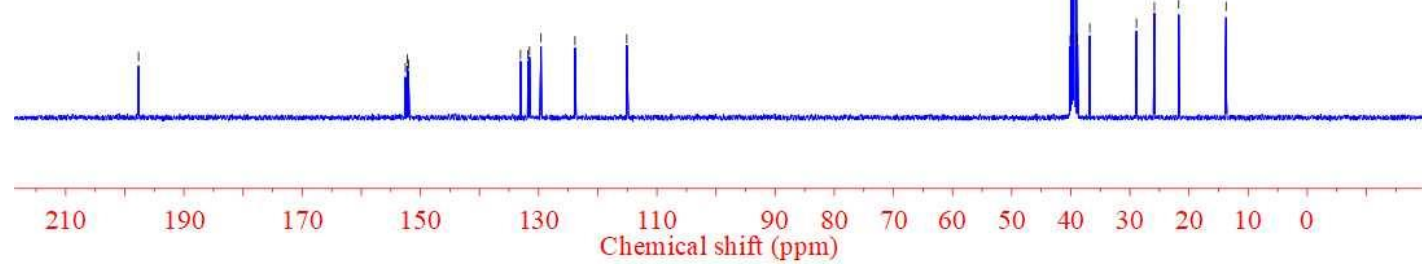

$33{ }^{1}$ H NMR (500 MHz, DMSO)

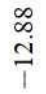

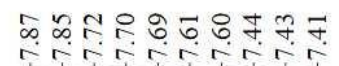

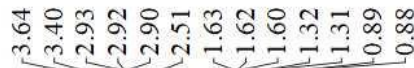<smiles>CCCCCC(=O)/C(=N/O)c1nc2ccccc2n(C)c1=O</smiles>

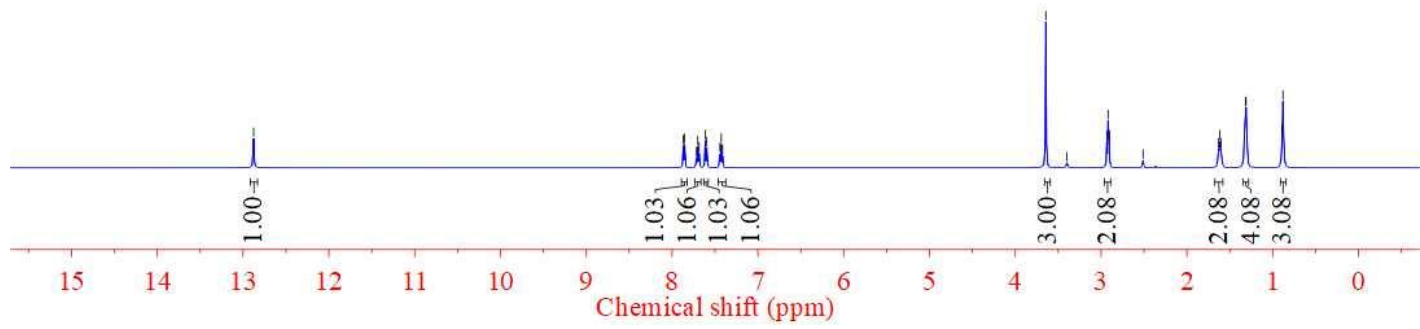


$33{ }^{13} \mathrm{C}$ NMR (126 MHz, DMSO)

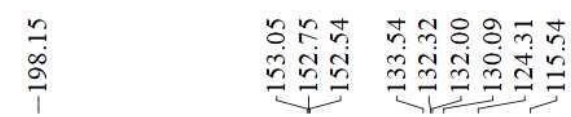

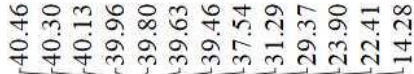<smiles>CCCCCC(=O)/C(=N/O)c1nc2ccccc2n(C)c1=O</smiles>
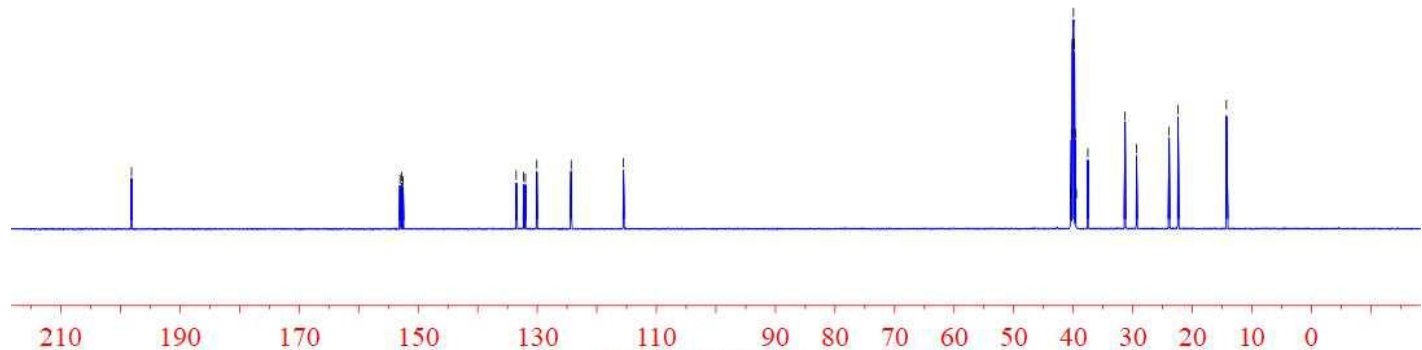

130

110

$90 \quad 80$

$\begin{array}{llllllll}70 & 60 & 50 & 40 & 30 & 20 & 10 & 0\end{array}$

\section{$34{ }^{1} \mathrm{H}$ NMR (500 MHz, DMSO)}

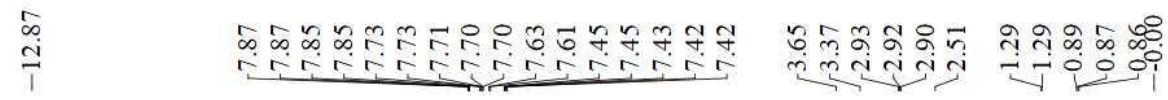<smiles>CCCCCCC(=O)/C(=N/O)c1nc2ccccc2n(C)c1=O</smiles>

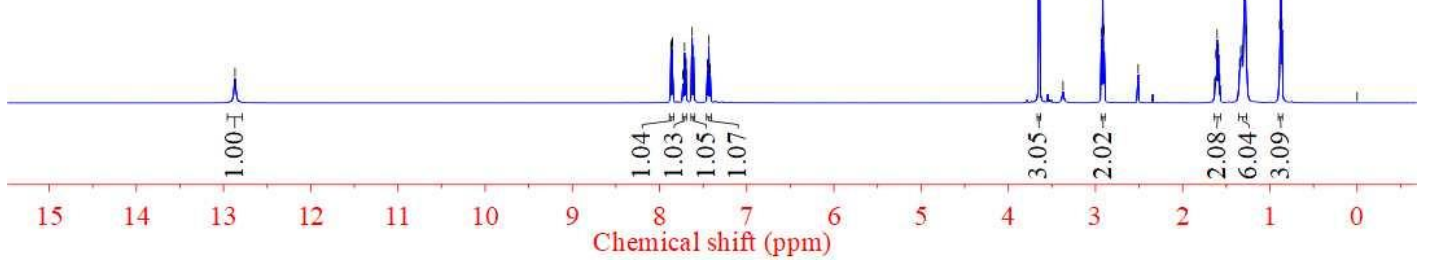


$34{ }^{13} \mathrm{C}$ NMR (101 MHz, DMSO)

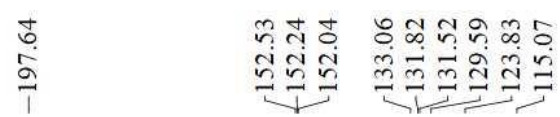

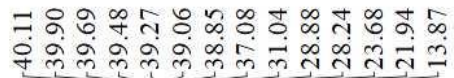<smiles>CCCCCCC(=O)/C(=N/O)c1nc2ccccc2n(C)c1=O</smiles>

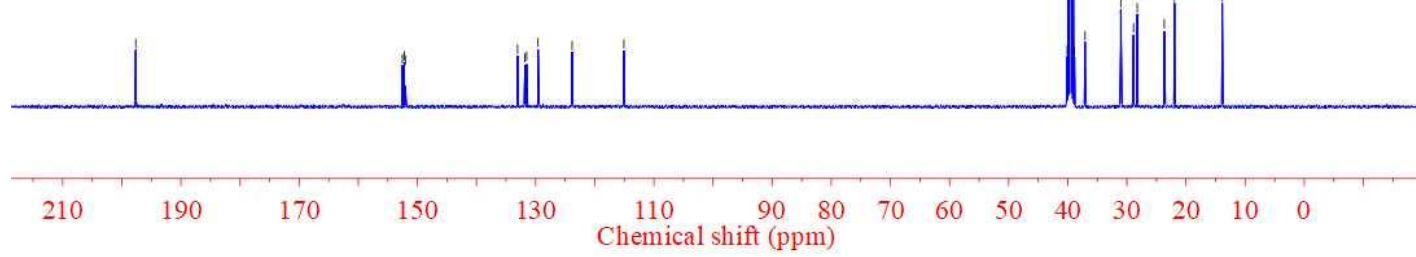

$35{ }^{1}$ H NMR (500 MHz, DMSO)

1
क

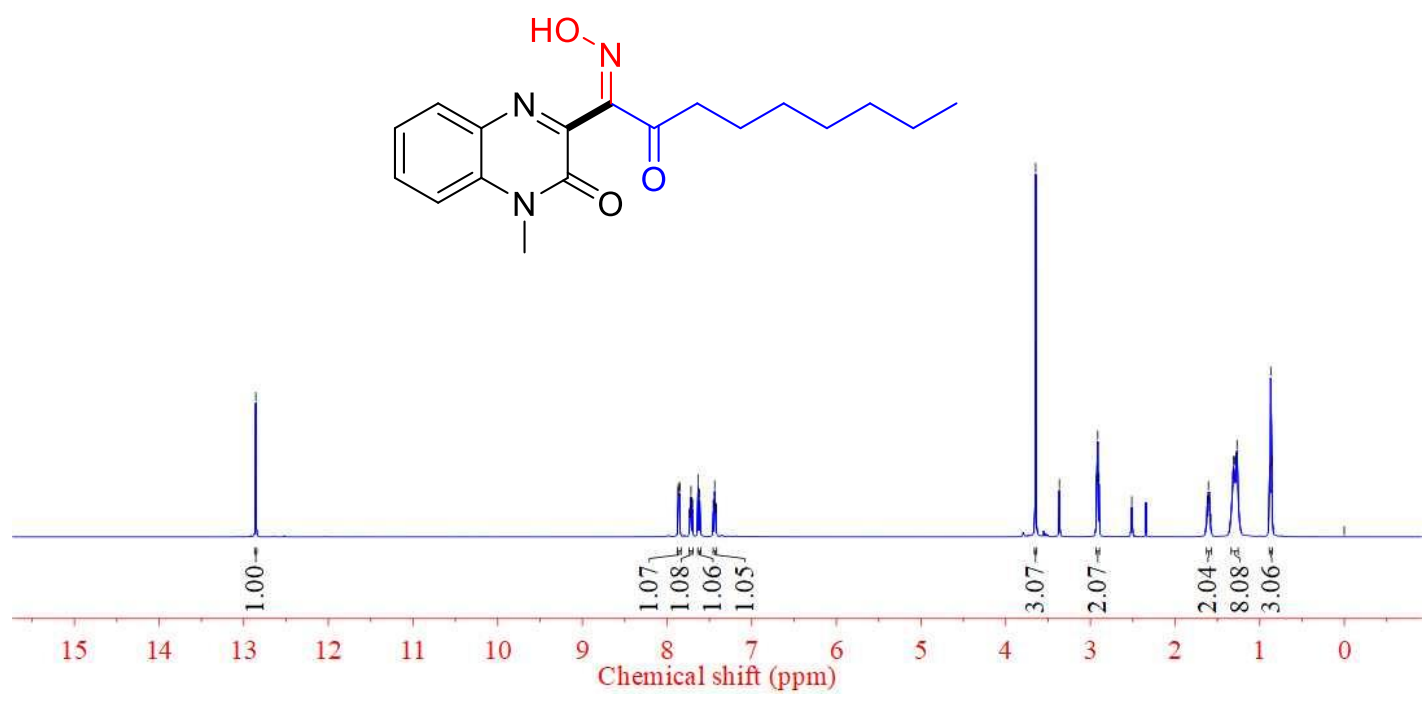


$35{ }^{13} \mathrm{C}$ NMR (101 MHz, DMSO)

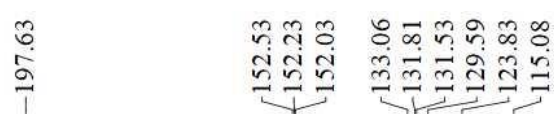

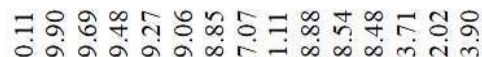

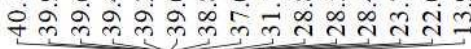<smiles>CCCCCCCC(=O)/C(=N/O)c1nc2ccccc2n(C)c1=O</smiles>

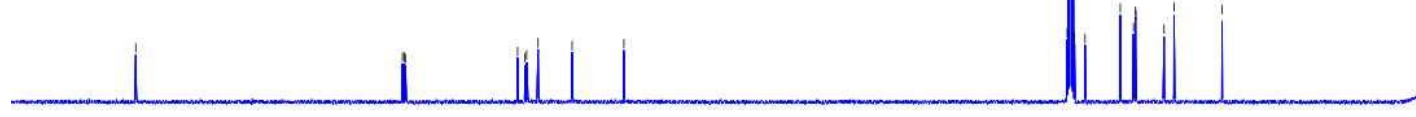

$210 \quad 190 \quad 170 \quad 150 \quad 130 \begin{gathered}110 \\ \text { Chemical shift (ppm) }\end{gathered}$

\section{$36{ }^{1} \mathrm{H}$ NMR (500 MHz, DMSO)}

$\stackrel{\grave{s}}{\grave{1}}$

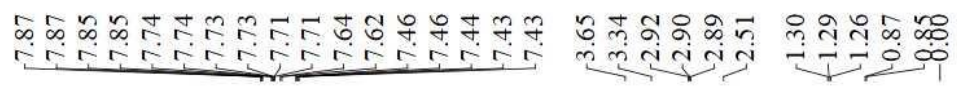<smiles>CCCCCCCCCC(=O)/C(=N/O)c1nc2ccccc2n(C)c1=O</smiles>

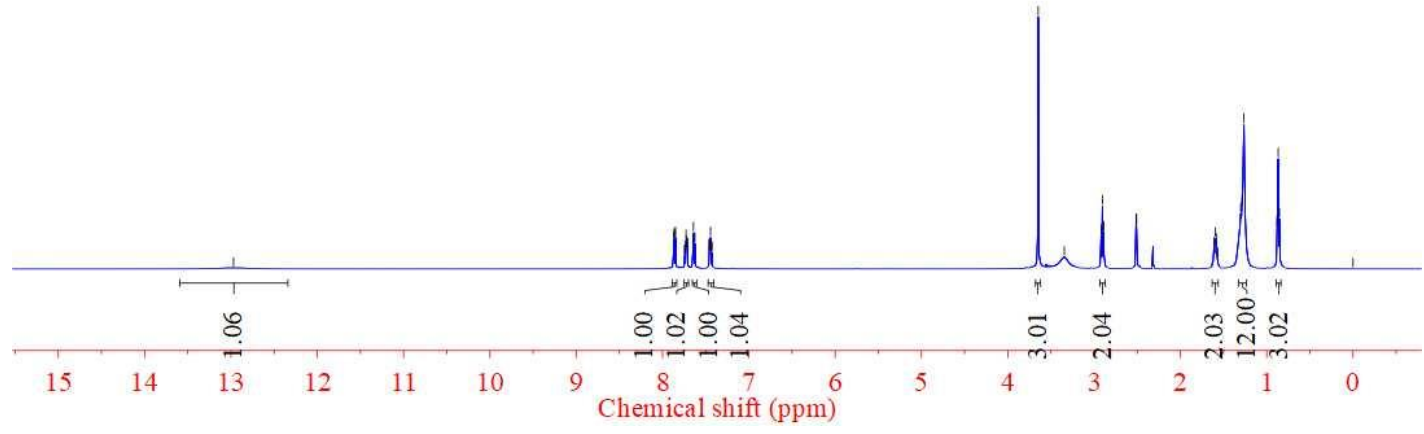




\section{$36{ }^{13} \mathrm{C}$ NMR (101 MHz, DMSO)}

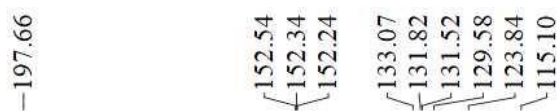

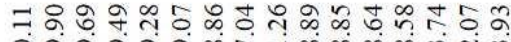

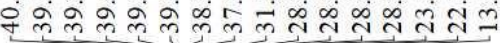<smiles>CCCCCCCCCC(=O)/C(=N/O)c1nc2ccccc2n(C)c1=O</smiles>

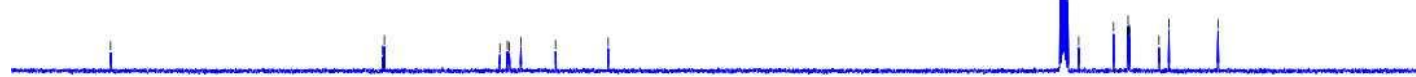

$210 \quad 190 \quad 170 \quad 150 \quad 130 \quad \begin{array}{cccccccccccc}110 & 90 & 80 & 70 & 60 & 50 & 40 & 30 & 20 & 10 & 0\end{array}$

\section{$37{ }^{1}$ H NMR (500 MHz, DMSO)}

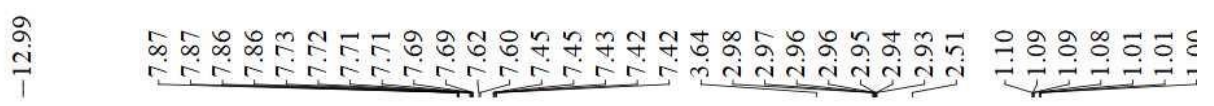<smiles>Cn1c(=O)c(/C(=N\O)C(=O)C2CC2)nc2ccccc21</smiles>

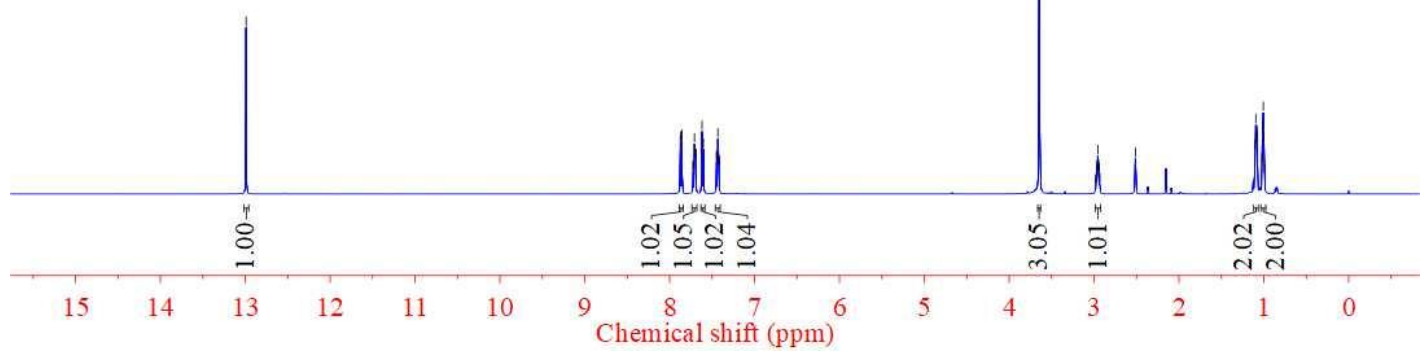


$37{ }^{13} \mathrm{C}$ NMR (126 MHz, DMSO)

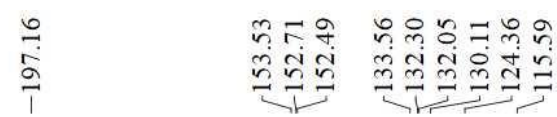

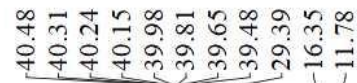

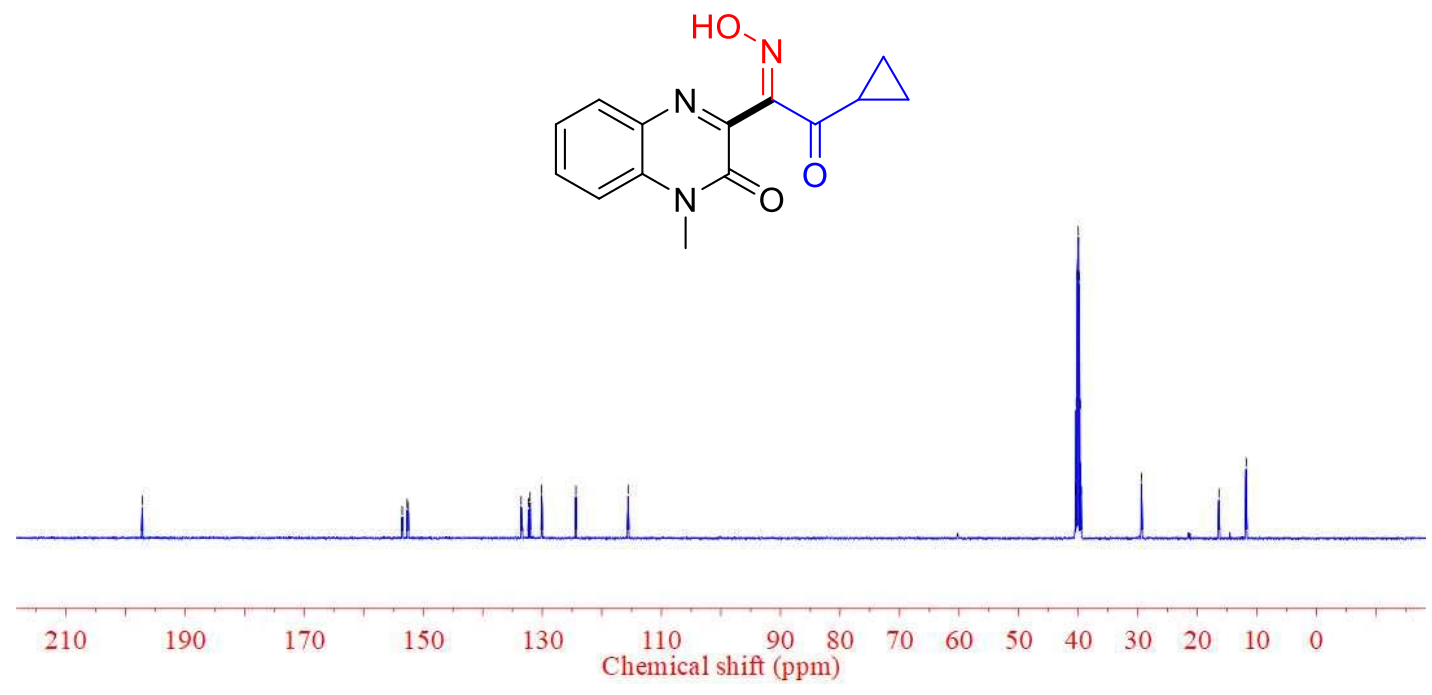

$38{ }^{1}$ H NMR (500 MHz, DMSO)

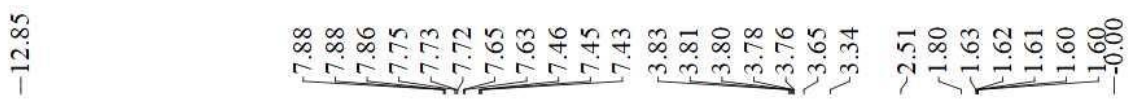<smiles>Cn1c(=O)c(C(=NO)C(=O)C2CCCC2)nc2ccccc21</smiles>

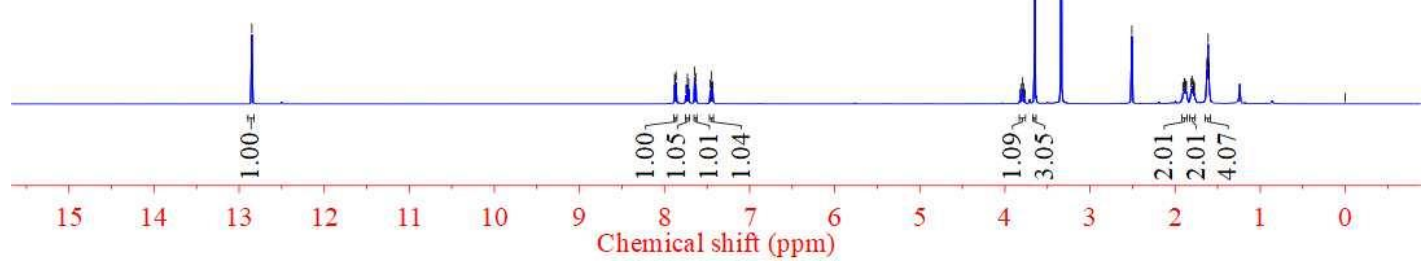


$38{ }^{13}$ C NMR (101 MHz, DMSO)

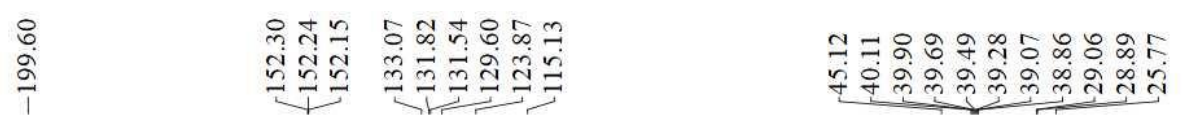<smiles>Cn1c(=O)c(/C(=N\O)C(=O)C2CCCC2)nc2ccccc21</smiles>

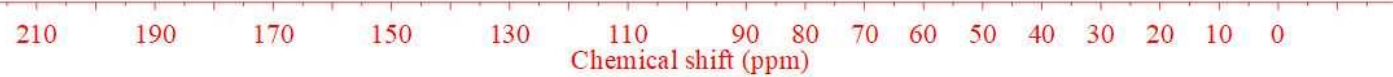

\section{$39{ }^{1} \mathrm{H}$ NMR (500 MHz, DMSO)}

1
0

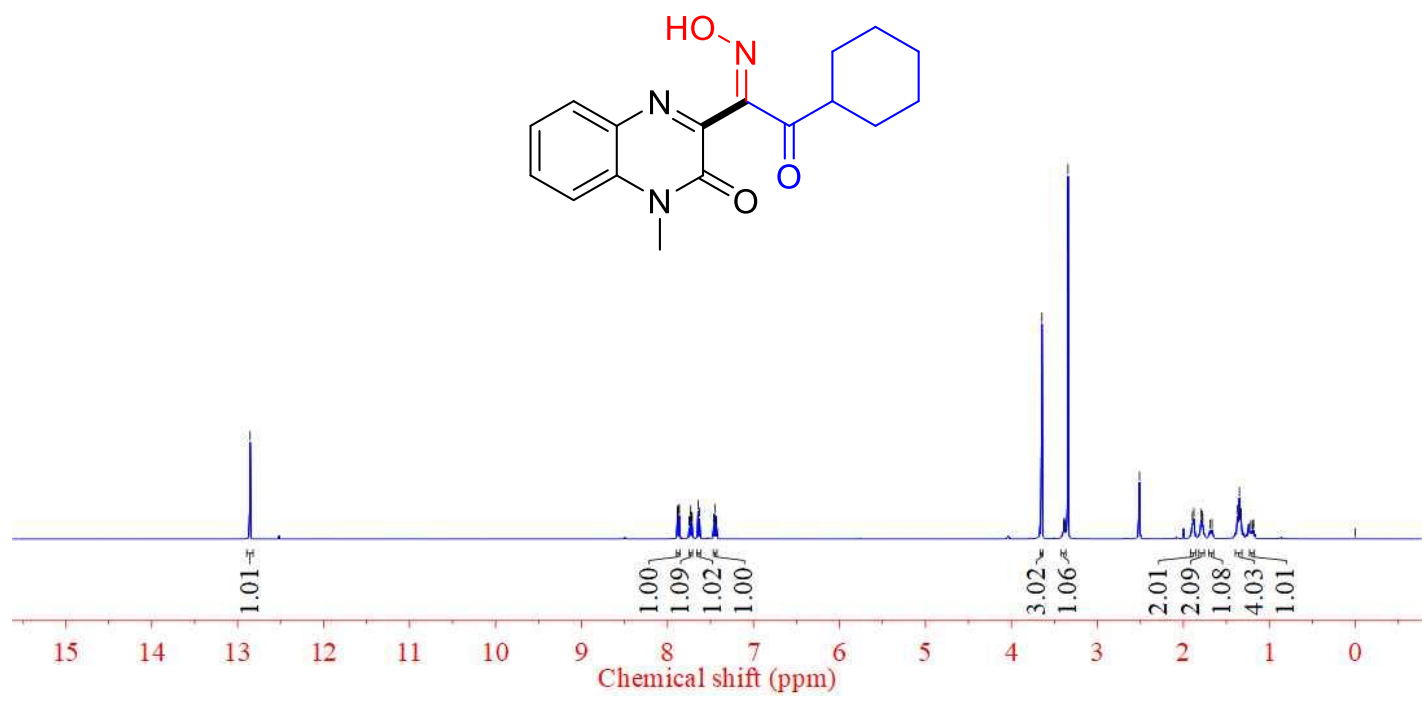


$39{ }^{13} \mathrm{C}$ NMR (101 MHz, DMSO)

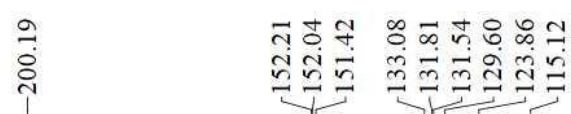

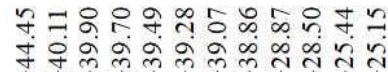<smiles>Cn1c(=O)c(C(=NO)C(=O)C2CCCCC2)nc2ccccc21</smiles>

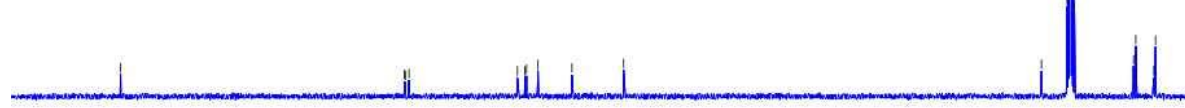

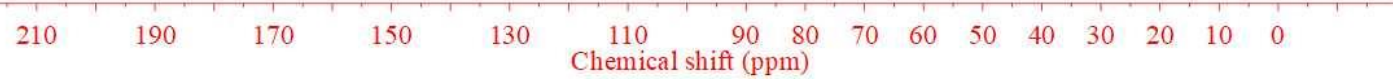

\section{$40{ }^{1} \mathrm{H}$ NMR (500 MHz, DMSO)}

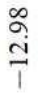

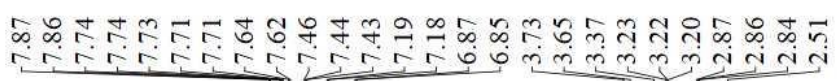<smiles>COc1ccc(CCC(=O)/C(=N/O)c2nc3ccccc3n(C)c2=O)cc1</smiles>

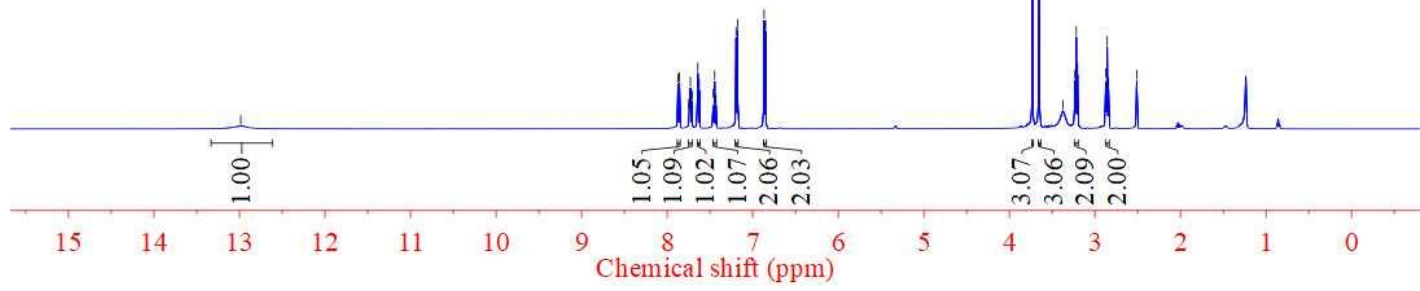


$40{ }^{13} \mathrm{C}$ NMR (126 MHz, DMSO)

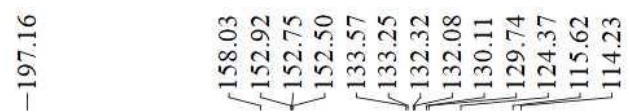

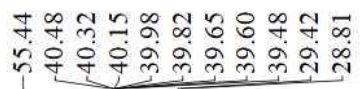<smiles>COc1ccc(CCC(=O)/C(=N/O)c2nc3ccccc3n(C)c2=O)cc1</smiles>
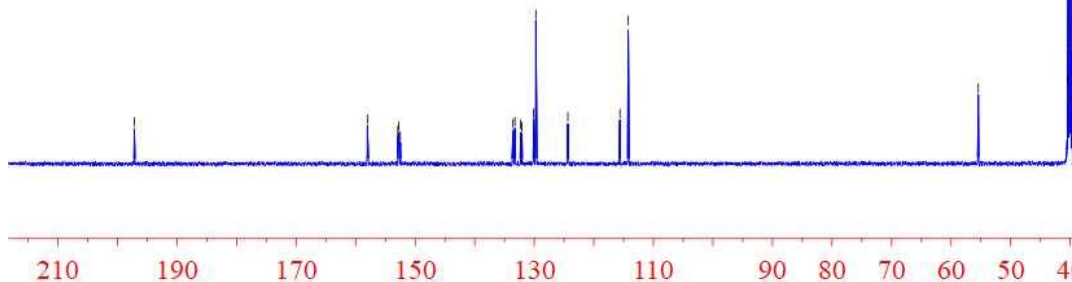

130

110
Chemical shift (ppm)

\section{$41{ }^{1}$ H NMR (500 MHz, DMSO)}

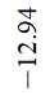

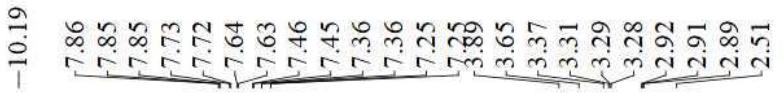<smiles>COc1cc(CCC(=O)C(=NO)c2nc3ccccc3n(C)c2=O)ccc1O</smiles>

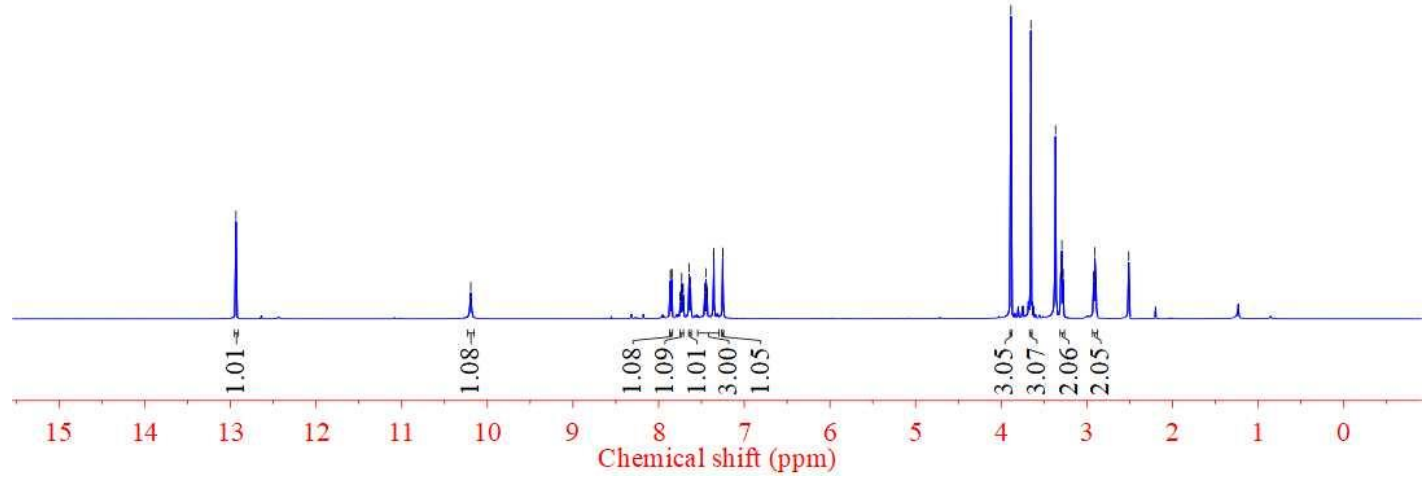


$41{ }^{13} \mathrm{C}$ NMR (126 MHz, DMSO)

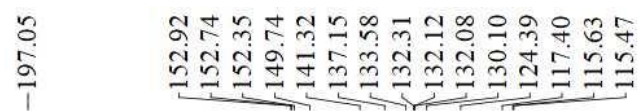

ప웅

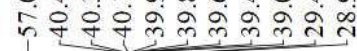<smiles>COc1cc(CCC(=O)/C(=N/O)c2nc3ccccc3n(C)c2=O)ccc1O</smiles>

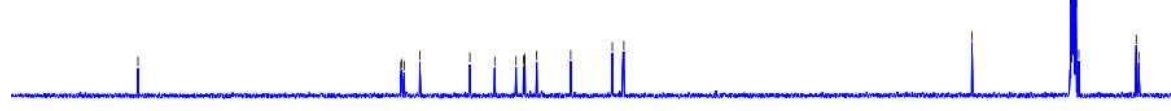

$210 \quad 190 \quad 170 \quad 150 \quad 130 \begin{gathered}110 \\ \text { Chemical shift (ppm) }\end{gathered}$

\section{$42{ }^{1} \mathrm{H}$ NMR (500 MHz, DMSO)}

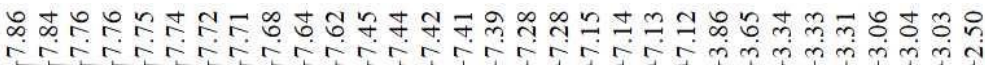<smiles>COc1ccc2cc(CCC(=O)C(=NO)c3nc4ccccc4n(C)c3=O)ccc2c1</smiles>

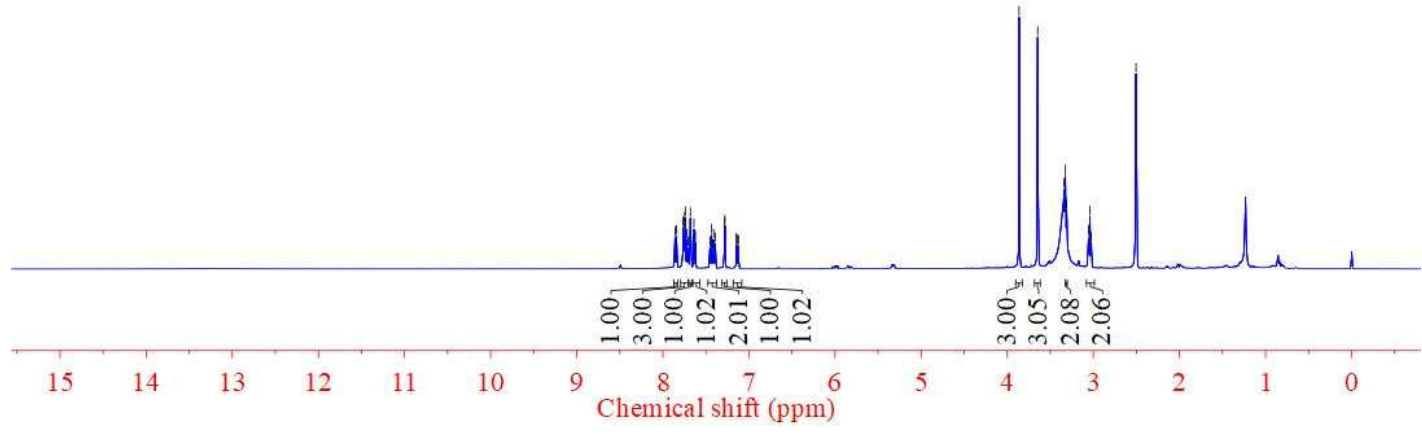




\section{$42{ }^{13} \mathrm{C}$ NMR (126 MHz, DMSO)}

$\stackrel{0}{\frac{1}{2}}$

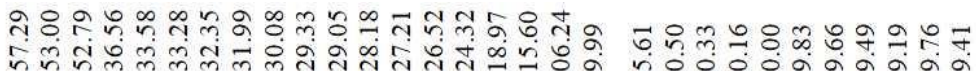

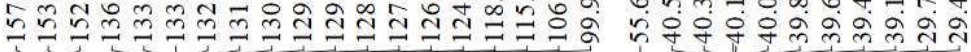<smiles>COc1ccc2cc(CCC(=O)C(=NO)c3nc4ccccc4n(C)c3=O)ccc2c1</smiles>

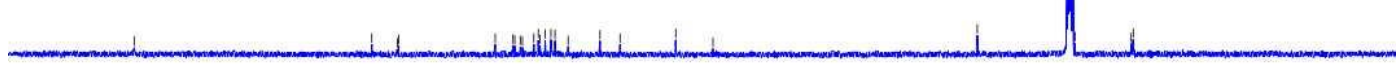

$210 \quad 190$

170

150

130

110

$90 \quad 80$

Chemical shift (ppm)

\section{$43{ }^{1}$ H NMR (500 MHz, DMSO)}

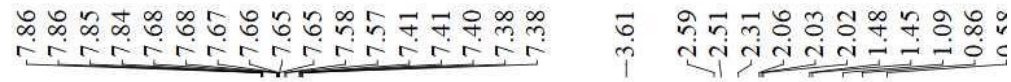<smiles>Cn1c(=O)c(/C(=N\O)C(=O)[C@H]2CC[C@]3(C)C4CC[C@H]5CC(=O)CC[C@]5(C)[C@H]4CC[C@]23C)nc2ccccc21</smiles>

$\mathrm{HO}^{-}$

$$
\text { (1) }
$$

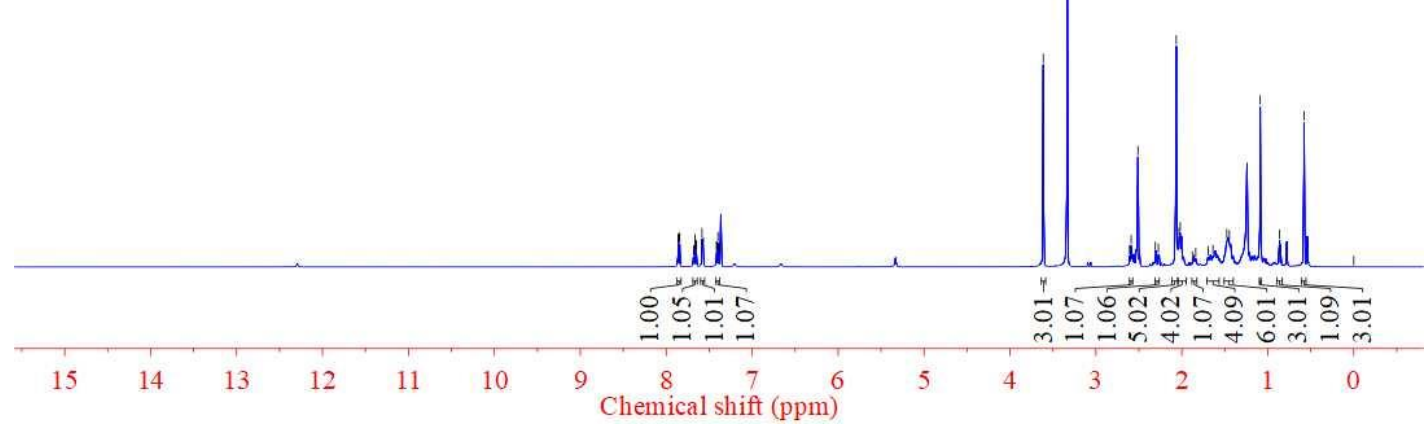


$43{ }^{13} \mathrm{C}$ NMR (126 MHz, DMSO)

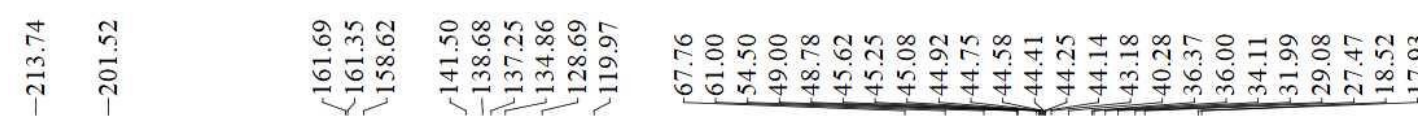

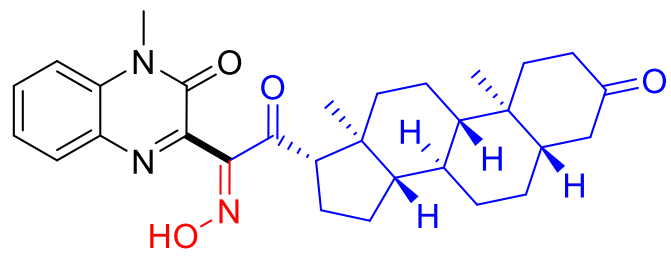

$210190 \quad 170 \quad 150 \quad 130 \begin{gathered}110 \\ \text { Chemical shift }(\mathrm{ppm})\end{gathered}$

\section{$44{ }^{1}$ H NMR (500 MHz, DMSO)}

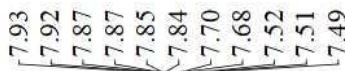

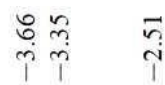<smiles>Cn1c(=O)c(C#N)nc2ccccc21</smiles>

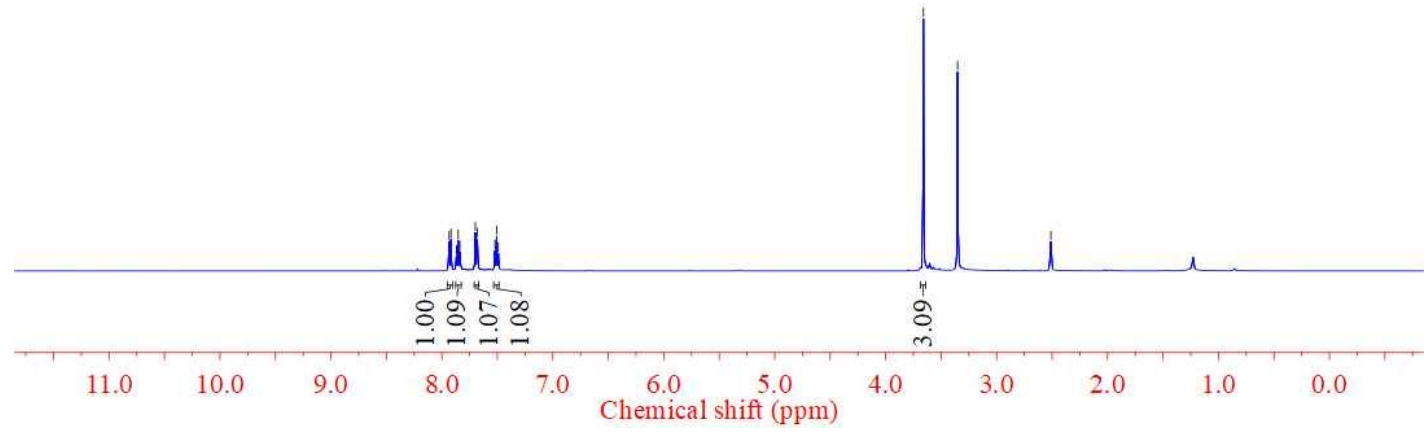


$44{ }^{13} \mathrm{C}$ NMR (126 MHz, DMSO)

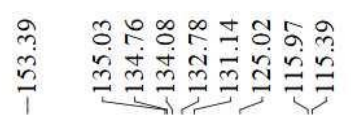

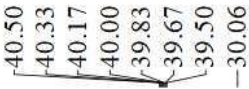<smiles>Cn1c(=O)c(C#N)nc2ccccc21</smiles>

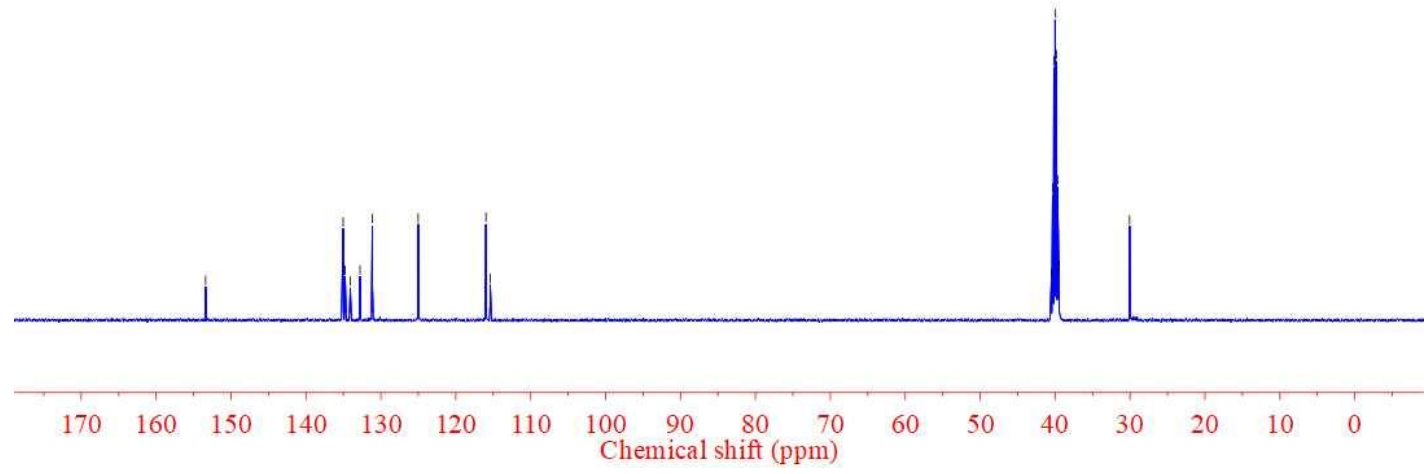

\section{$45{ }^{1} \mathrm{H}$ NMR (500 MHz, $\mathrm{CDCl}_{3}$ )}

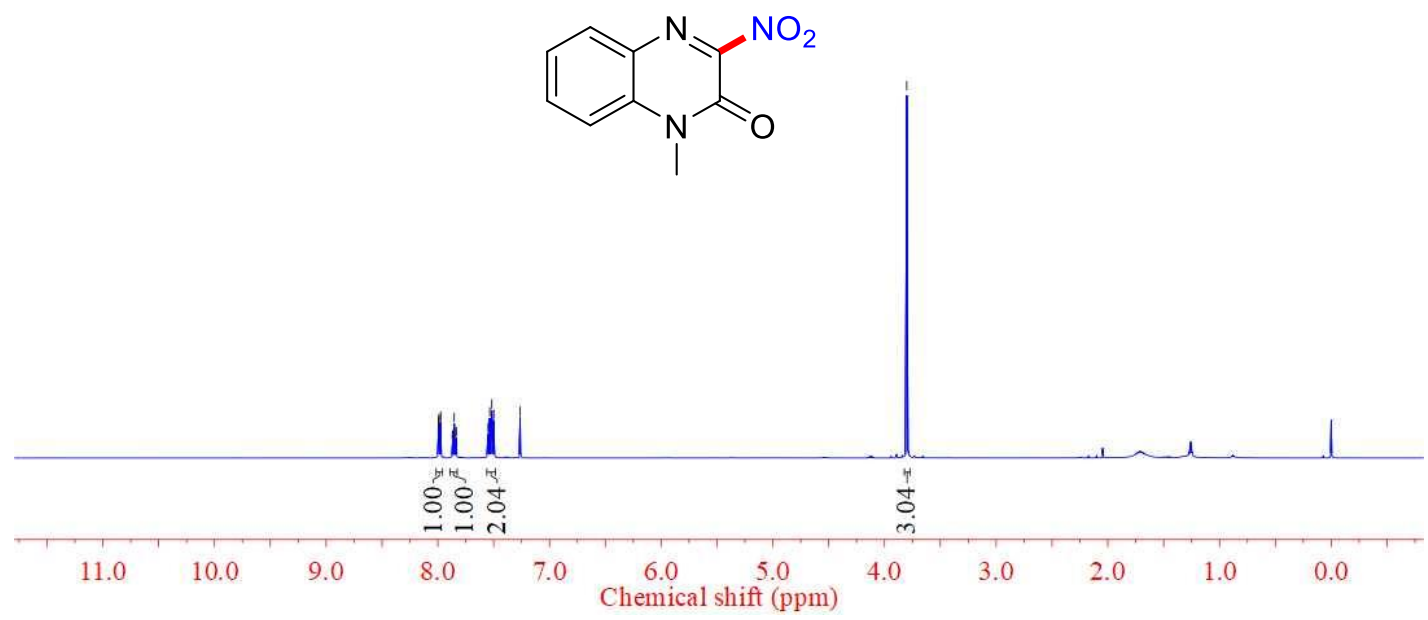


$45{ }^{13} \mathrm{C}$ NMR (126 MHz, $\left.\mathrm{CDCl}_{3}\right)$

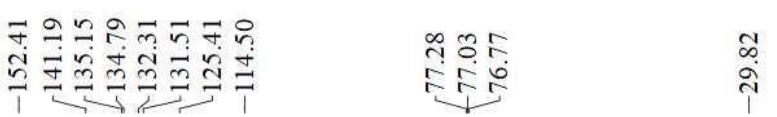

$\overbrace{\mathrm{O}}^{\mathrm{NO}_{2}}$

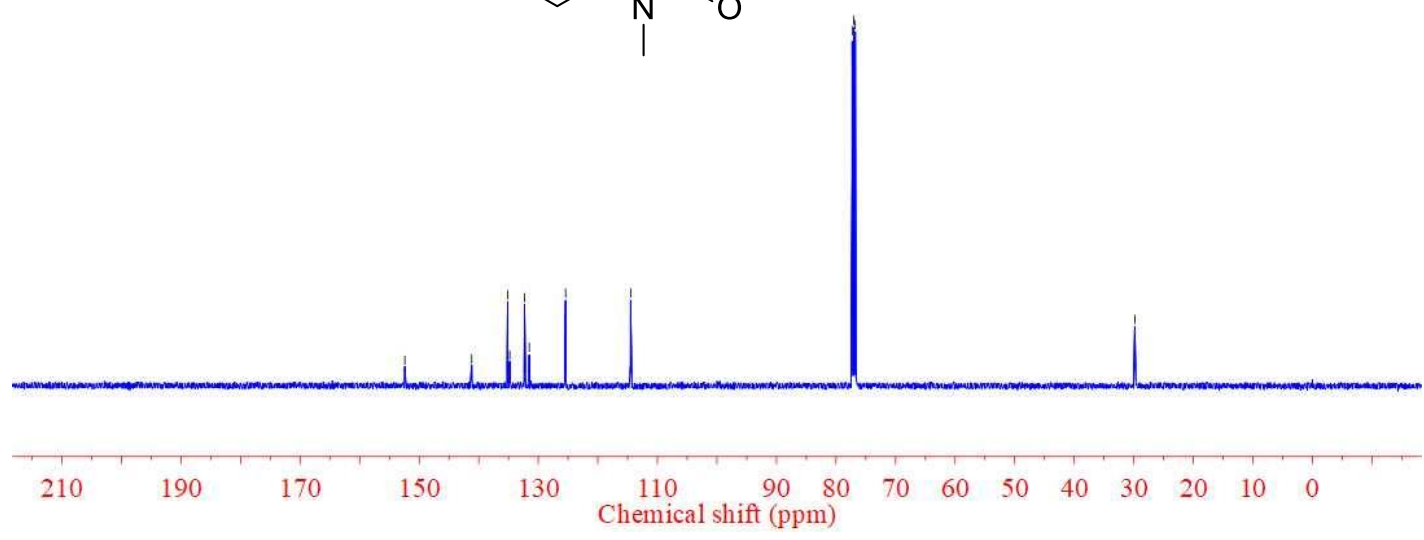

$46{ }^{1} \mathrm{H}$ NMR (500 MHz, $\left.\mathrm{CDCl}_{3}\right)$

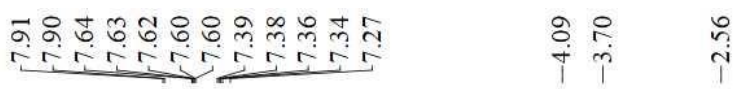

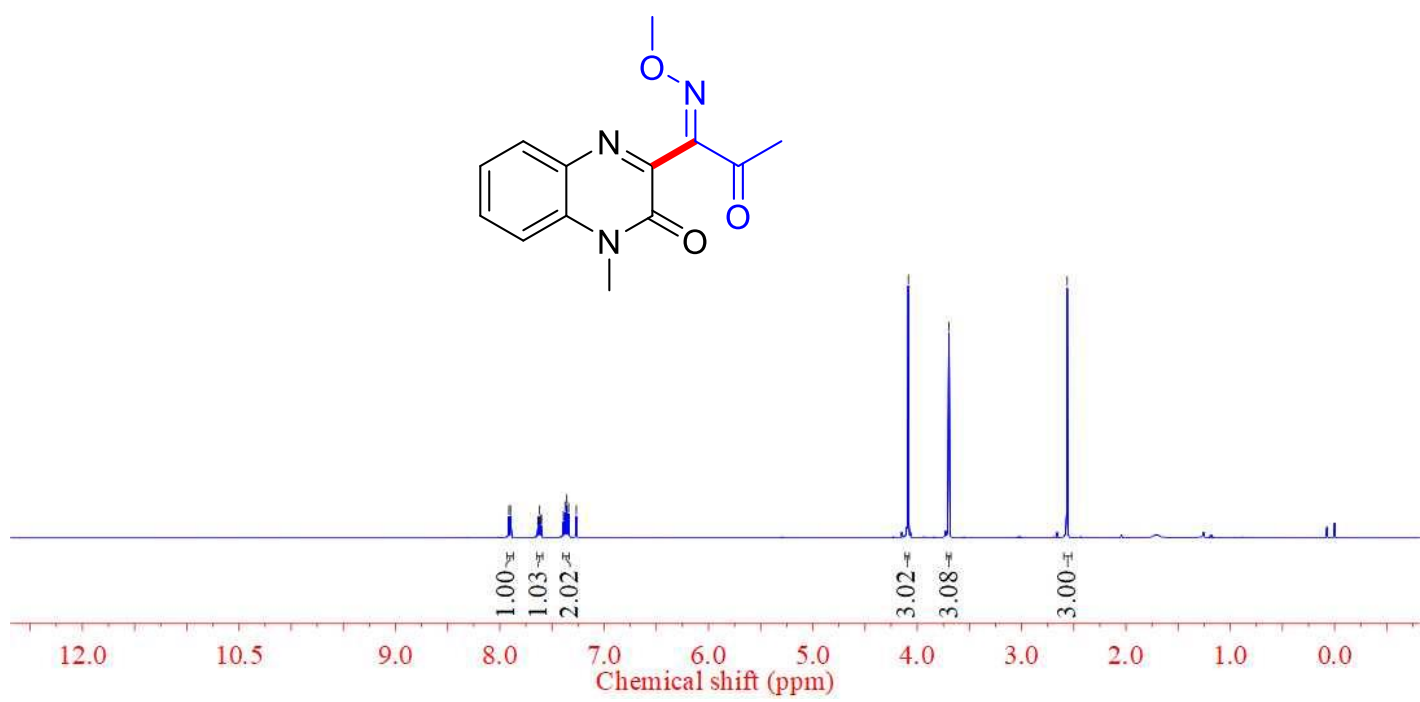


$46{ }^{13} \mathrm{C}$ NMR (126 MHz, $\left.\mathrm{CDCl}_{3}\right)$

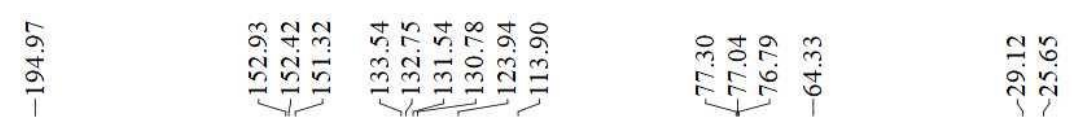<smiles>CO/N=C(/C(C)=O)c1nc2ccccc2n(C)c1=O</smiles>

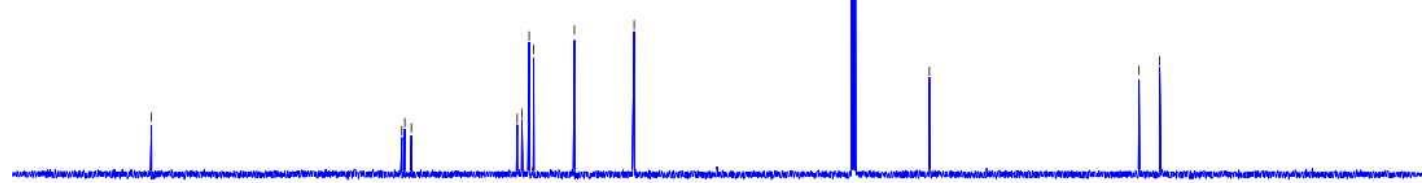

210

$190 \quad 170 \quad 150$

130

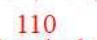

Chemical shift (ppm)

\section{$47^{1} \mathrm{H}$ NMR (500 MHz, $\left.\mathrm{CDCl}_{3}\right)$}

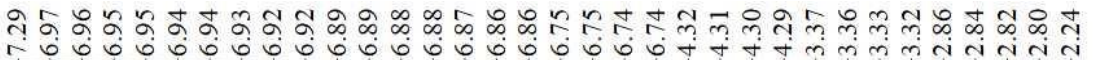

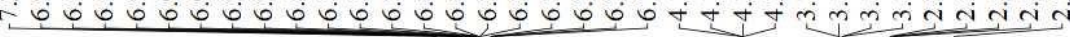

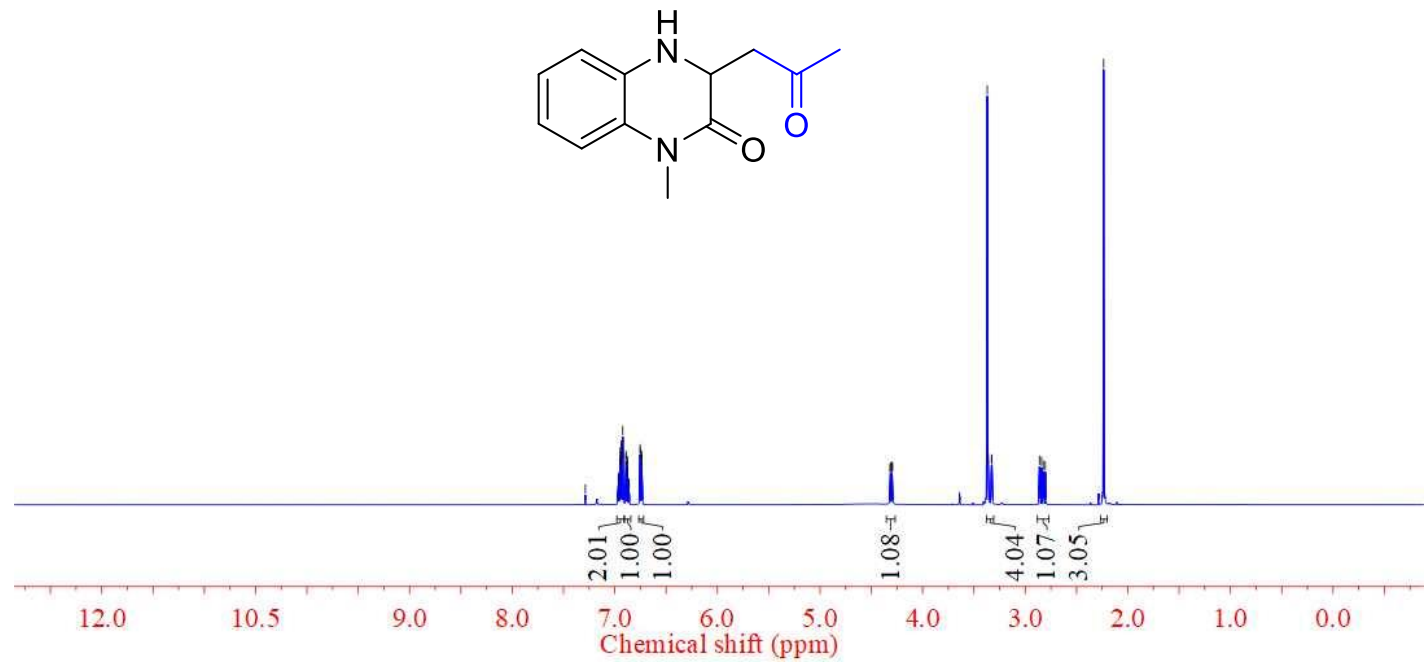


$47{ }^{13} \mathrm{C}$ NMR (126 MHz, $\left.\mathrm{CDCl}_{3}\right)$

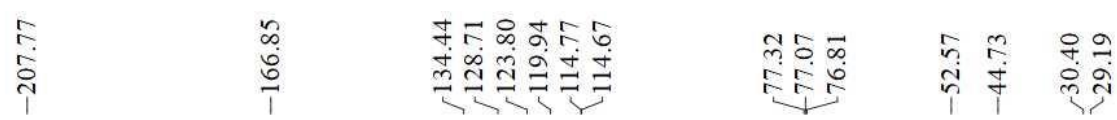<smiles>CC(=O)CC1Nc2ccccc2N(C)C1=O</smiles>

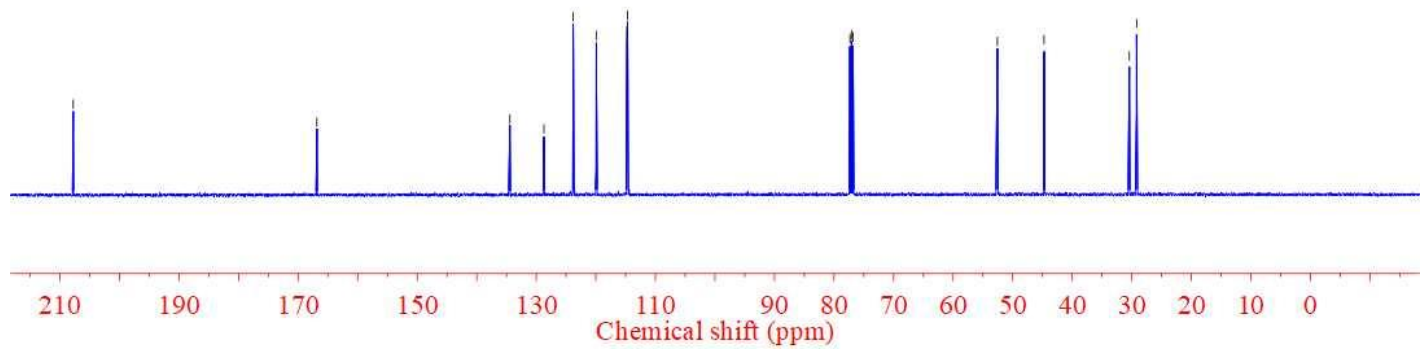

$48{ }^{1} \mathrm{H}$ NMR (500 MHz, $\left.\mathrm{CDCl}_{3}\right)$

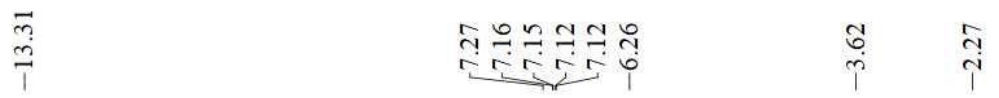<smiles>CC(=O)/C=C1/Nc2ccccc2N(C)C1=O</smiles>

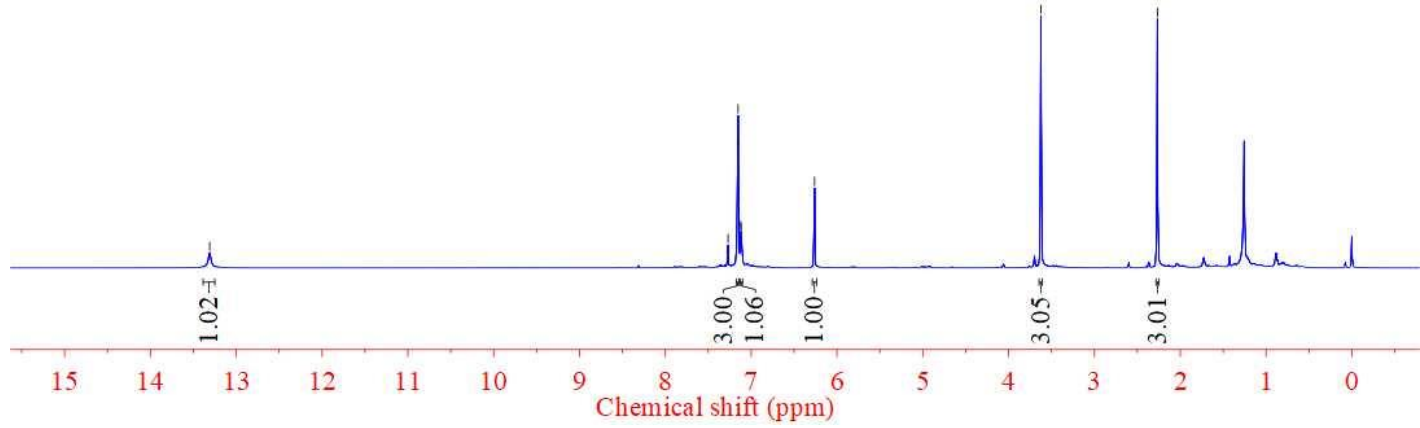


$48{ }^{13} \mathrm{C}$ NMR (126 MHz, $\left.\mathrm{CDCl}_{3}\right)$

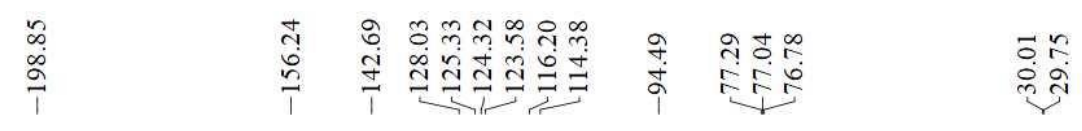
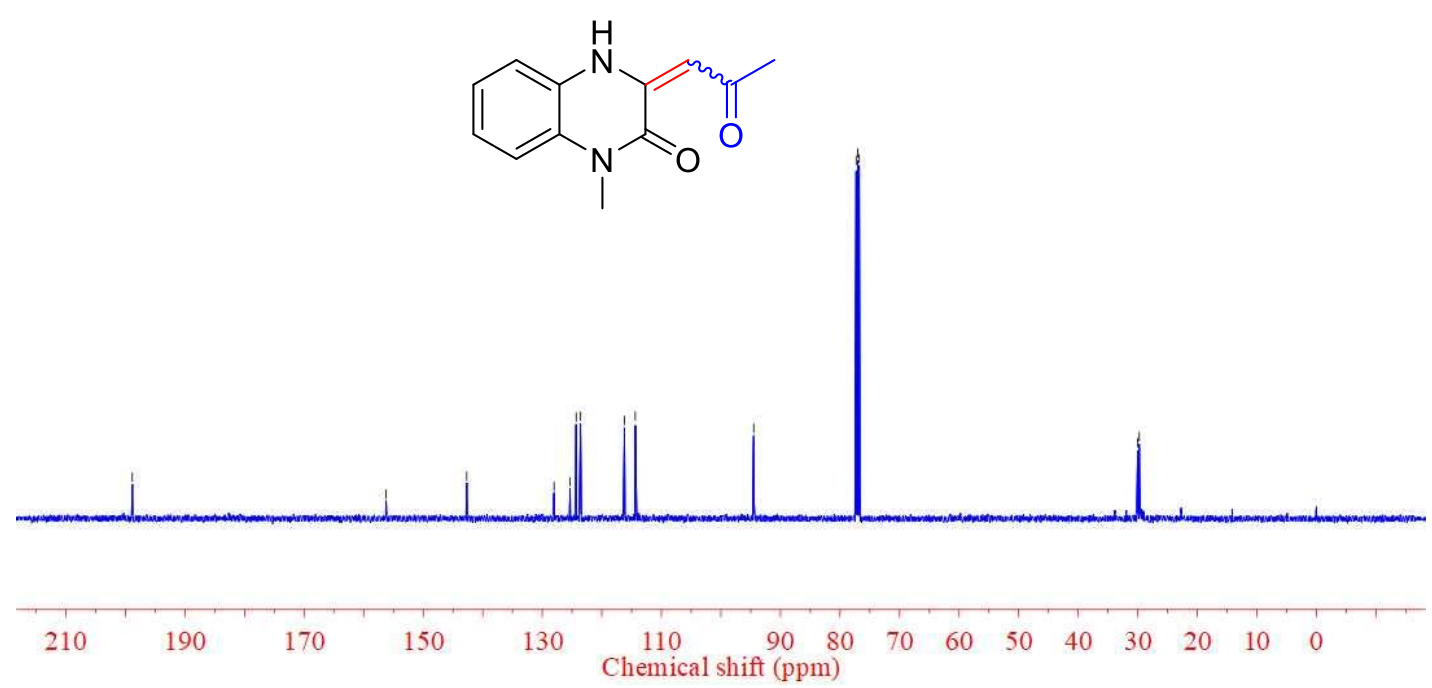

\section{$50{ }^{1} \mathrm{H}$ NMR (500 MHz, $\mathrm{CDCl}_{3}$ )}

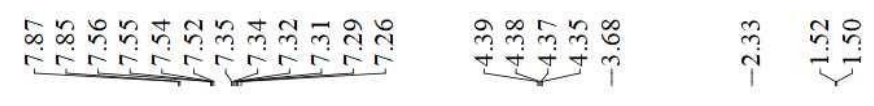<smiles>CC(=O)C(C)c1nc2ccccc2n(C)c1=O</smiles>

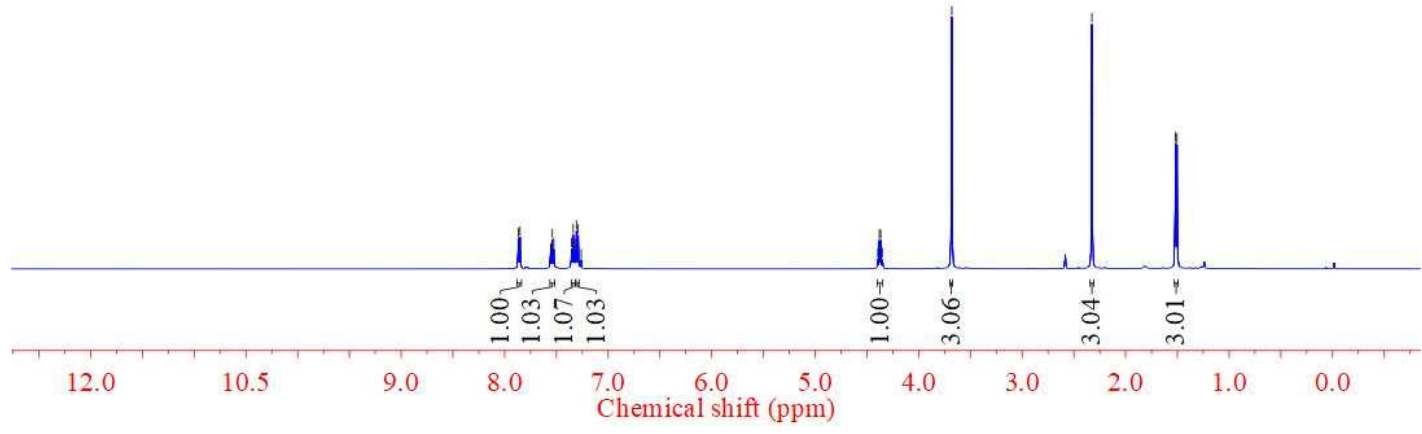


$50{ }^{13} \mathrm{C}$ NMR (126 MHz, $\left.\mathrm{CDCl}_{3}\right)$

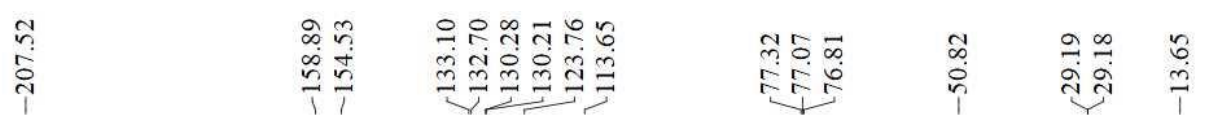<smiles>CC(=O)C(C)c1nc2ccccc2n(C)c1=O</smiles>

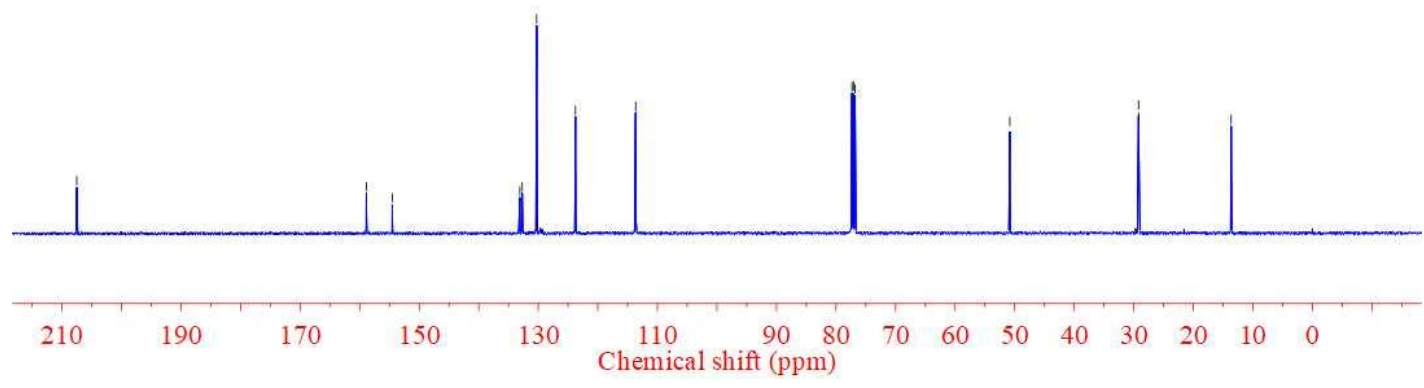

$51{ }^{1} \mathrm{H}$ NMR (500 MHz, $\left.\mathrm{CDCl}_{3}\right)$

ô om

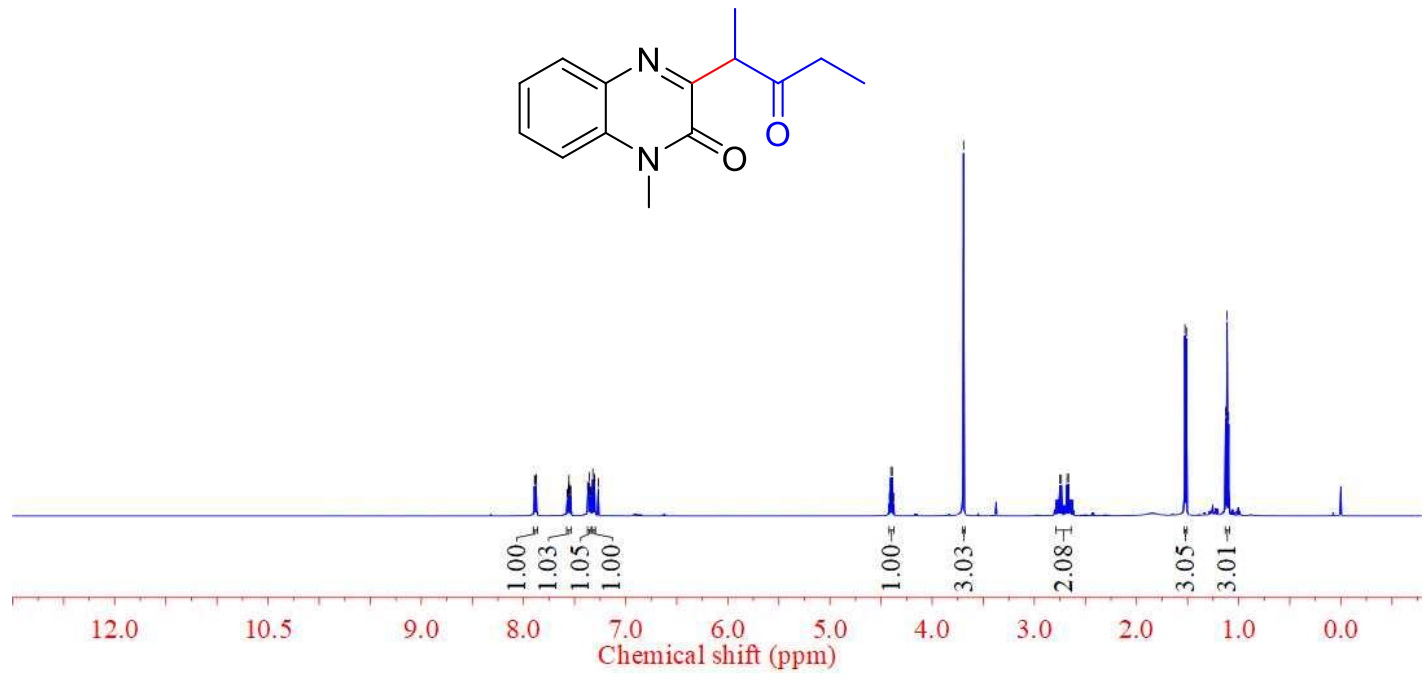




\section{$51{ }^{13} \mathrm{C}$ NMR (126 MHz, $\left.\mathrm{CDCl}_{3}\right)$}

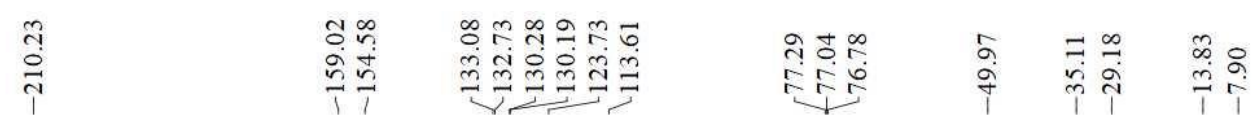<smiles>CCC(=O)C(C)c1nc2ccccc2n(C)c1=O</smiles>

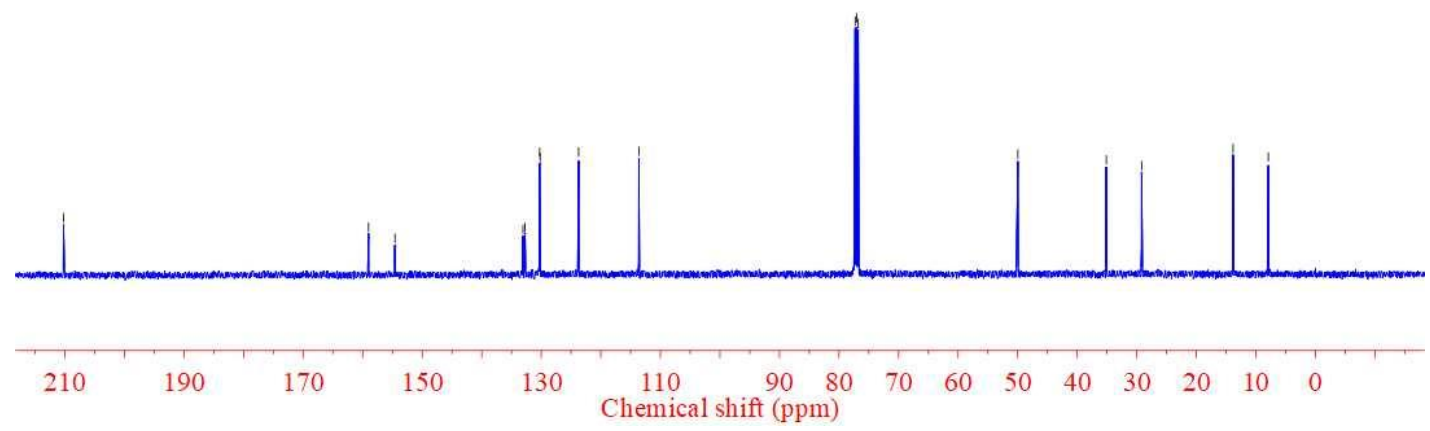




\section{Copies of HRMS Spectra}

3

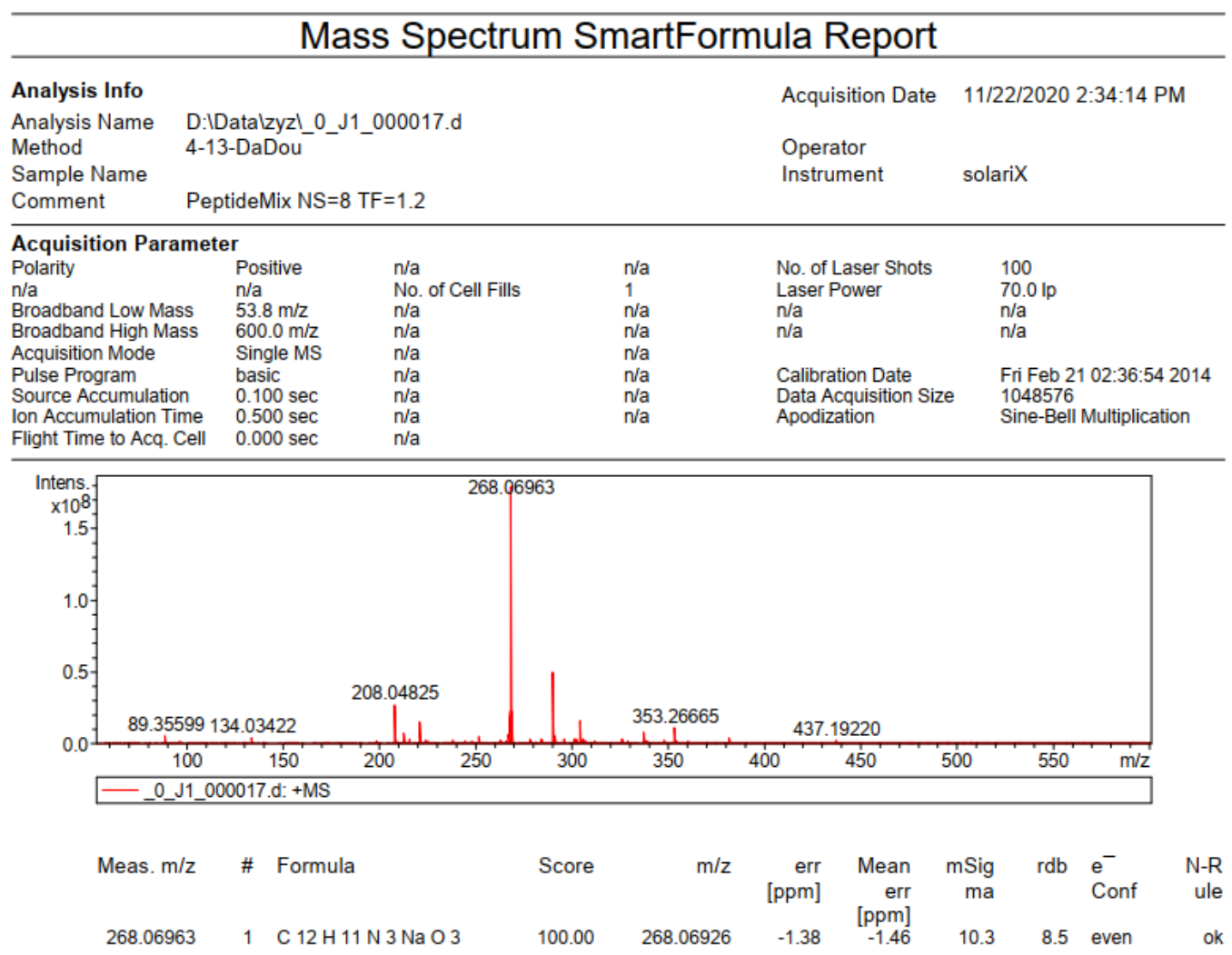

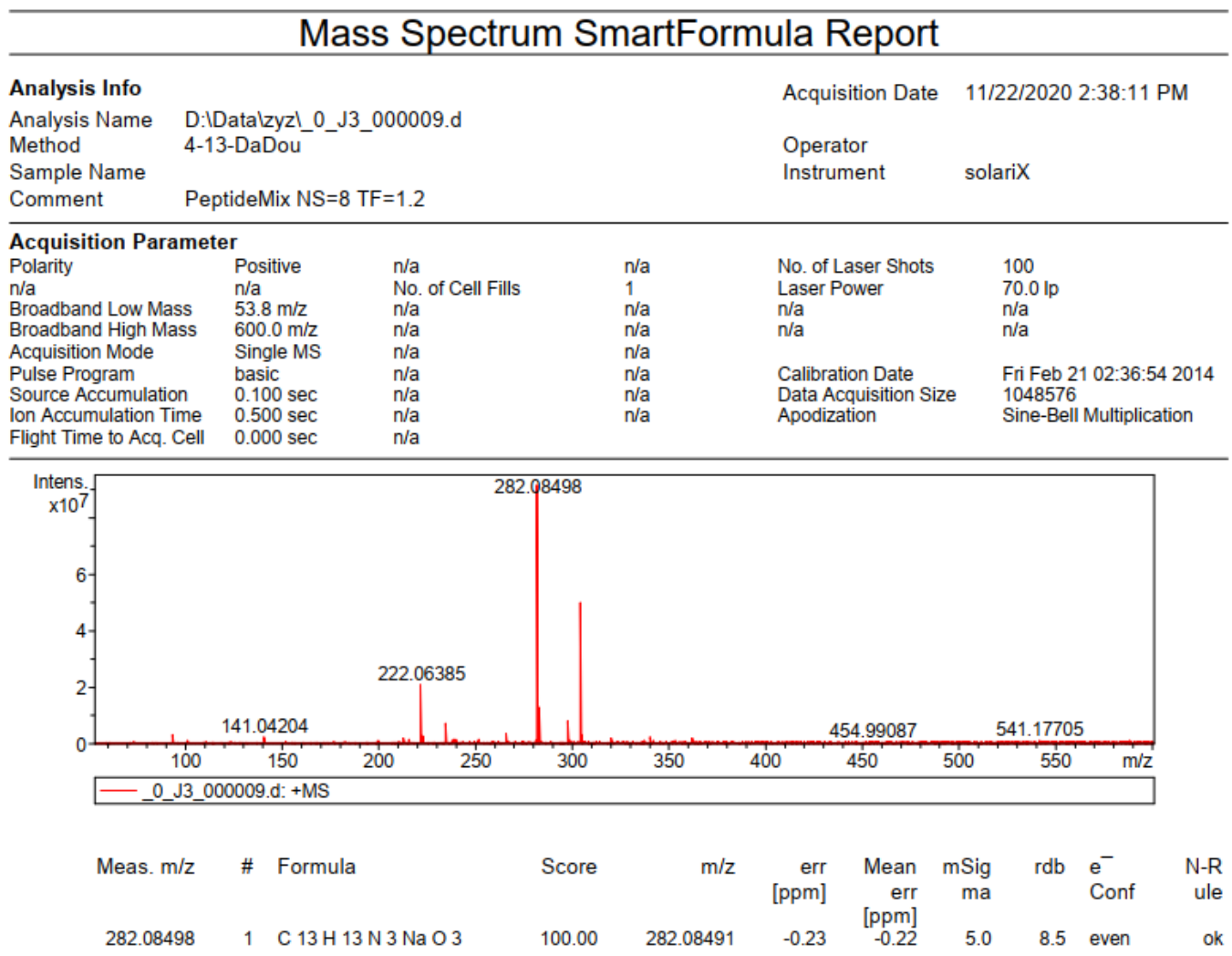




\section{Mass Spectrum SmartFormula Report}

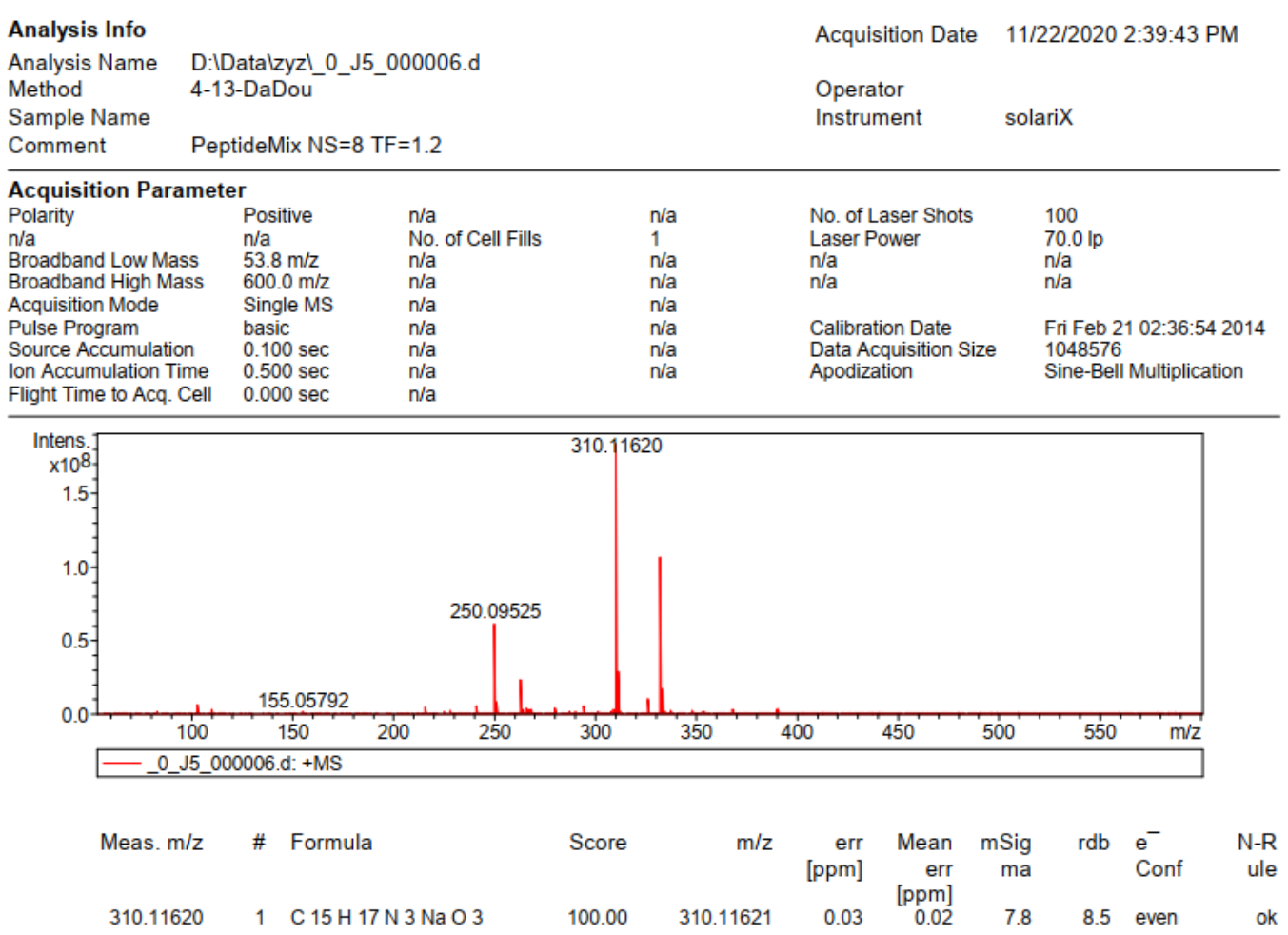

6

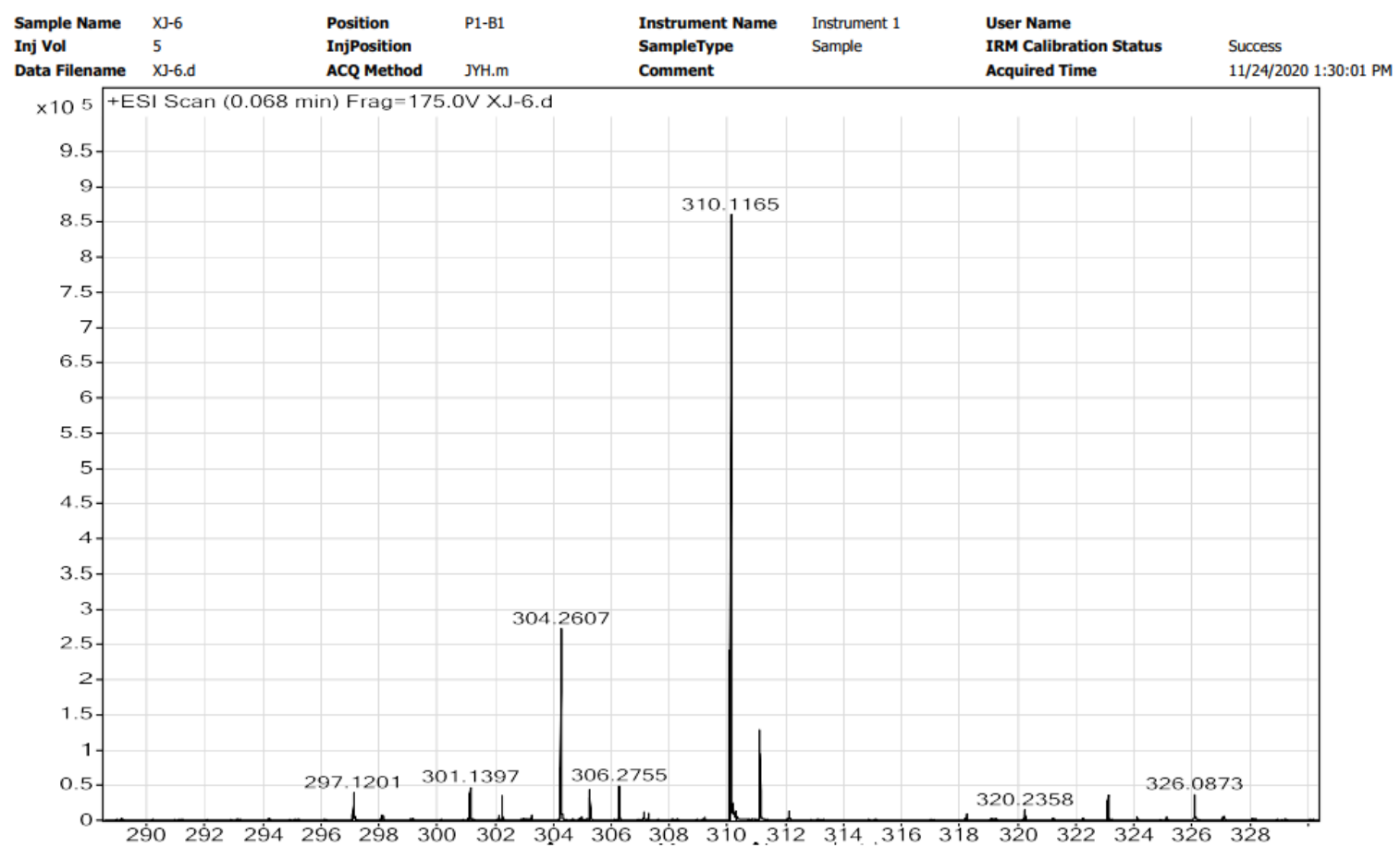

\begin{tabular}{|l|r|r|r|r|r|r|r|}
\hline Formula (M) & Score (MFG) & Mass & Mass (MFG) & $\mathrm{m} / \mathrm{z}$ (Calc) & Diff (ppm) & DBE & $\mathrm{m} / \mathrm{z}$ \\
\hline C15 H17 N3 O3 & 99.7 & 287.1273 & 287.127 & 310.1162 & -1 & 310.1165 \\
\hline
\end{tabular}




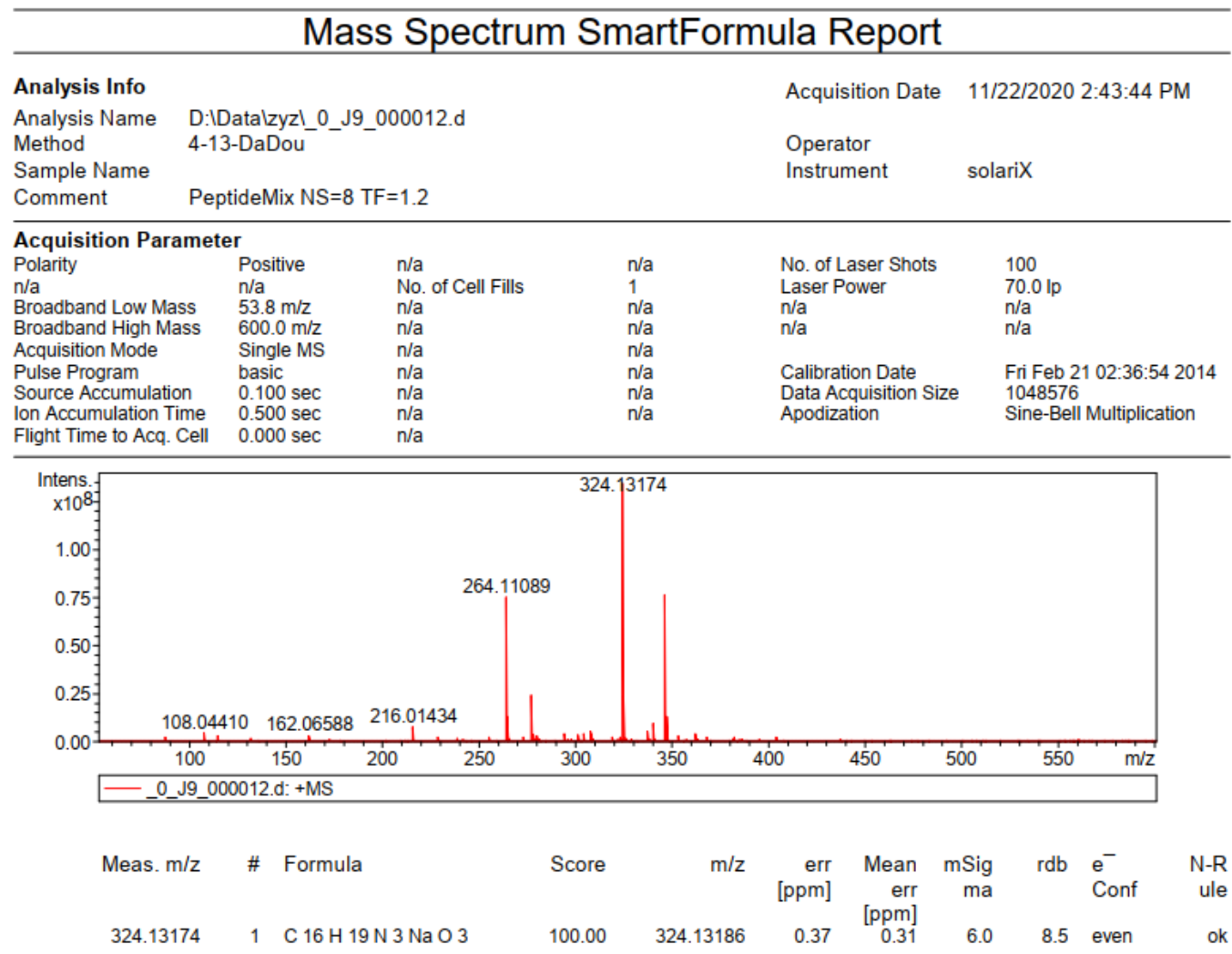

\section{Mass Spectrum SmartFormula Report}

Analysis Info

Analysis Name

Method

Sample Name

Comment

\section{Acquisition Paramete}

Parameter

Broadband Low Mass

Broadband Low Mass

Broadband High

Acquisition Mode

Source Accumulation

Flight Time to Acq. Cell

D:IDatalzyz!_0_J11_000007.d

4-13-DaDou

eptideMix NS=8 TF=1.2

Acquisition Date $\quad 11 / 22 / 2020$ 2:45:22 PM

Operator

Instrument solariX

\section{Positive $\quad \mathrm{n} / \mathrm{a}$}

n/a No. of Cell Fills

$53.8 \mathrm{~m} / \mathrm{z}$

Single MS

Single

$0.100 \mathrm{sec}$

$500 \mathrm{sec}$

$0.000 \mathrm{sec}$

Intens.

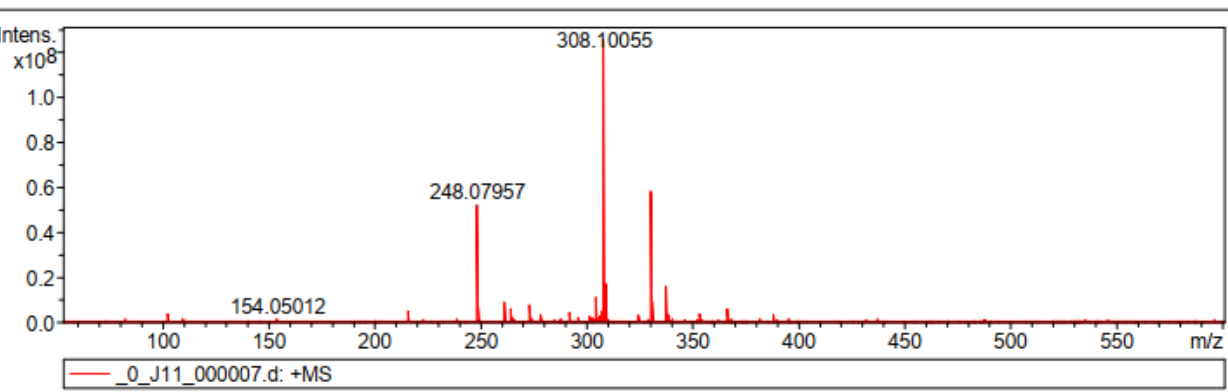

Meas. $\mathrm{m} / \mathrm{z}$

\# Formula

$n / a$

$n / a$
$n / a$

n/a

$\mathrm{n} / \mathrm{a}$

$\mathrm{n} / \mathrm{a}$

n/a

of Cell Fills

No. of Laser Shots

70.0 Ip

$\begin{array}{ll}\mathrm{n} / \mathrm{a} & \mathrm{n} / \mathrm{a} \\ \mathrm{n} / \mathrm{a} & \mathrm{n} / \mathrm{a}\end{array}$

Calibration Date

Data Acquisition Size

Fri Feb 21 02:36:54 2014

Sine-Bell Multiplication

$\begin{array}{lllrrrrrrr}\text { Meas. } \mathrm{m} / \mathrm{z} & \text { \# } & \text { Formula } & \text { Score } & \mathrm{m} / \mathrm{z} & \begin{array}{r}\text { err } \\ {[\mathrm{ppm}]}\end{array} & \begin{array}{r}\text { Mean } \\ \text { err } \\ {[\mathrm{ppm}]}\end{array} & \begin{array}{r}\mathrm{mSig} \\ \mathrm{ma}\end{array} & \begin{array}{r}\mathrm{rdb} \\ \mathrm{e}^{-}\end{array} & \begin{array}{r}\mathrm{N}-\mathrm{R} \\ \text { Conf }\end{array} \\ \text { ule }\end{array}$




\section{Mass Spectrum SmartFormula Report}

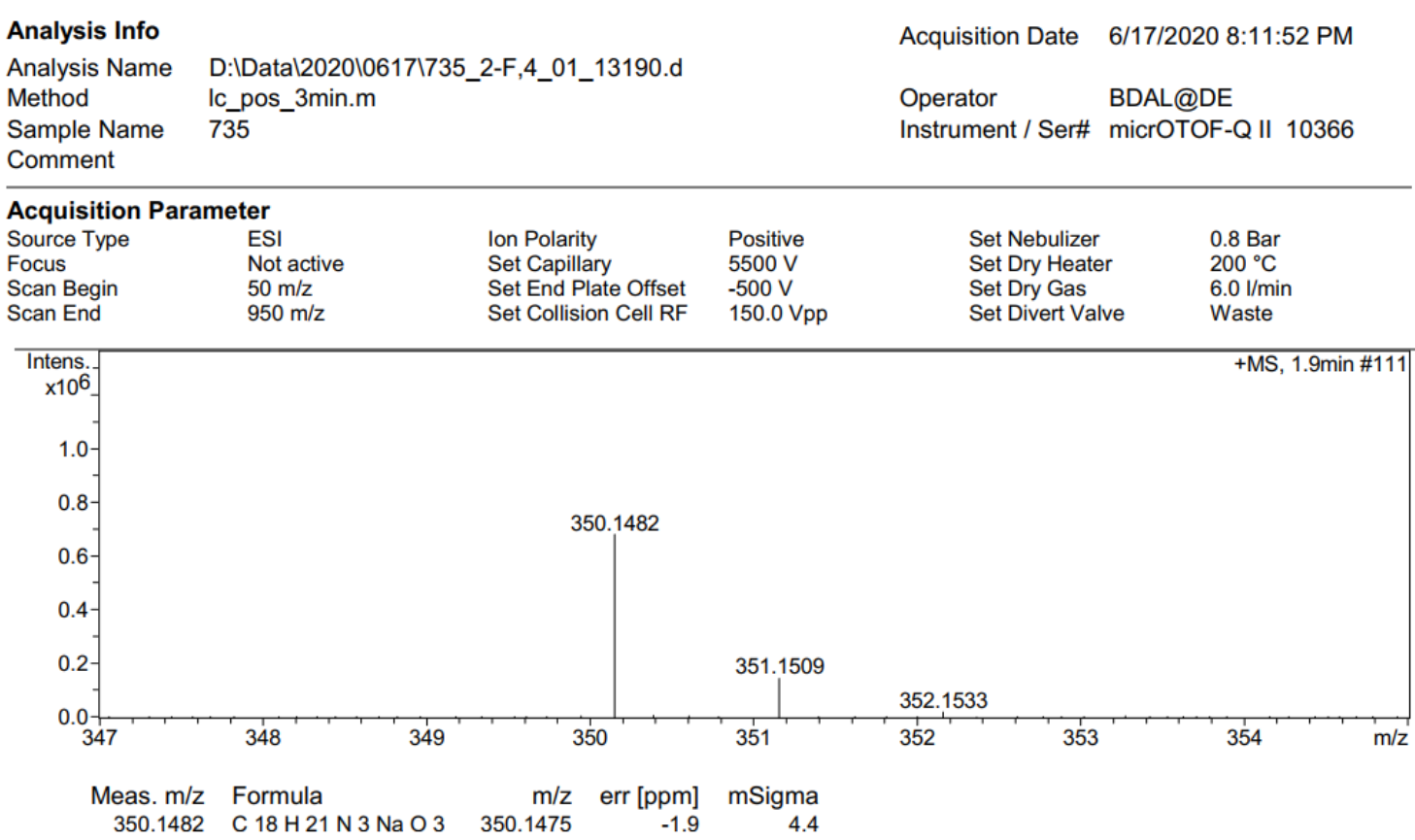

10

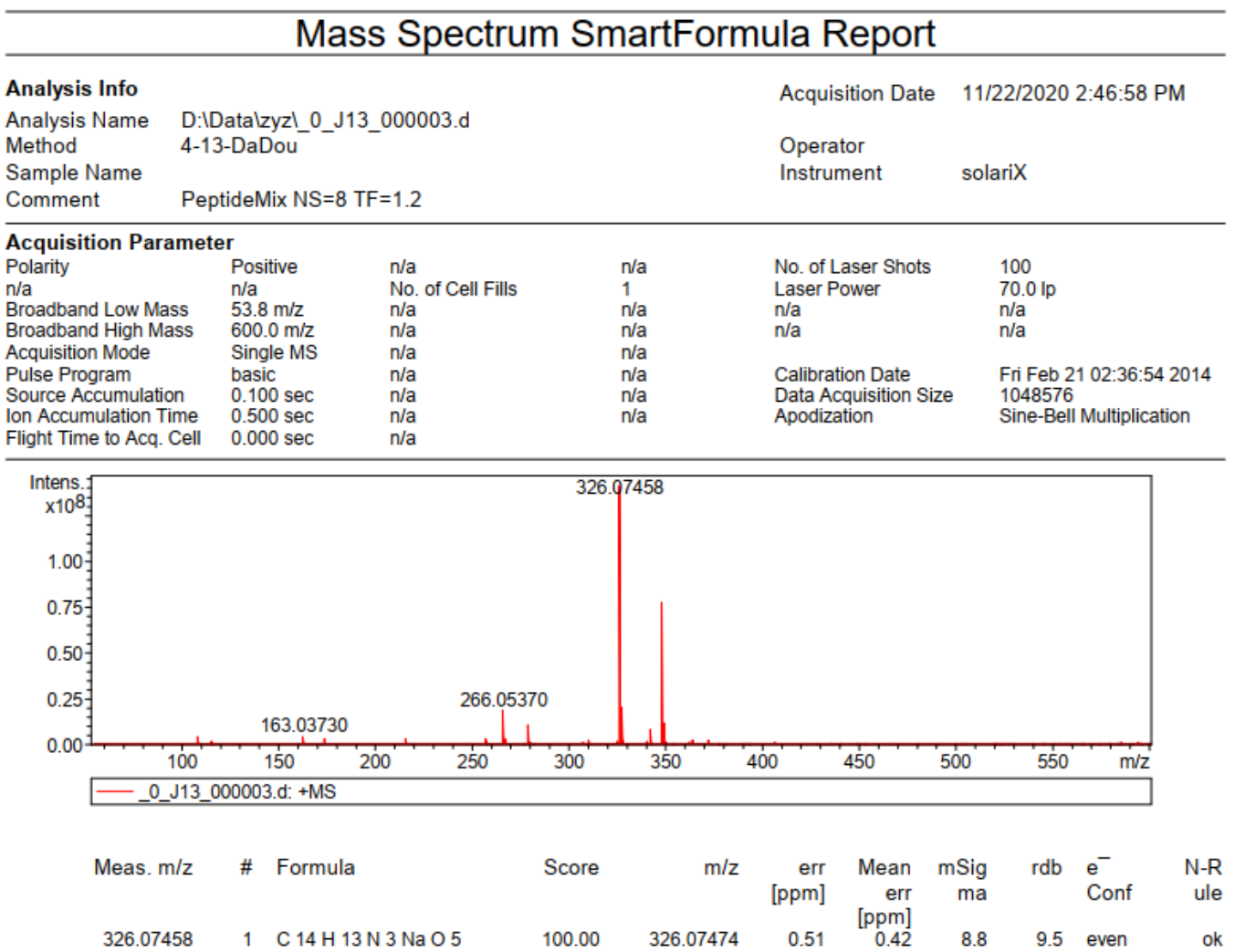




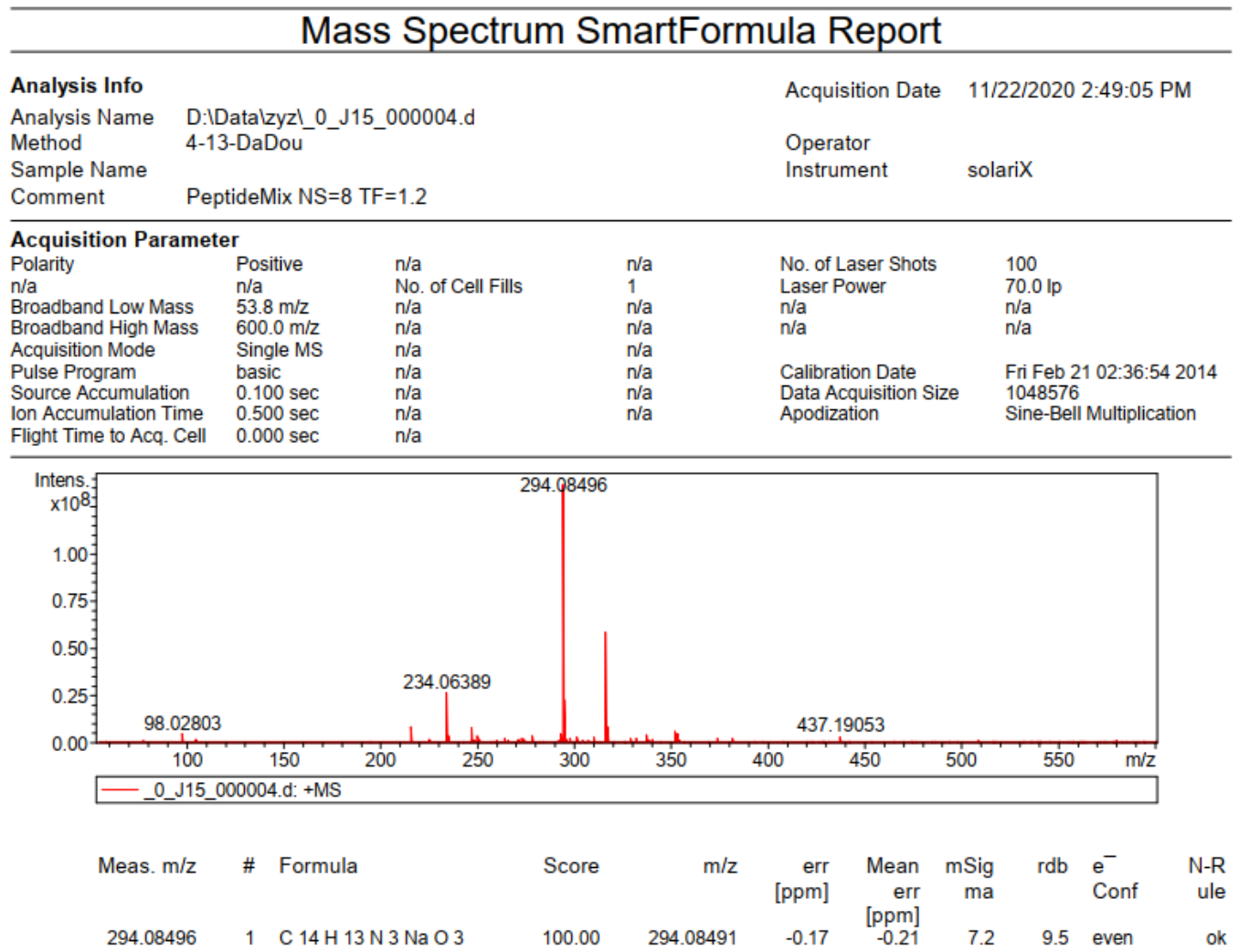

12

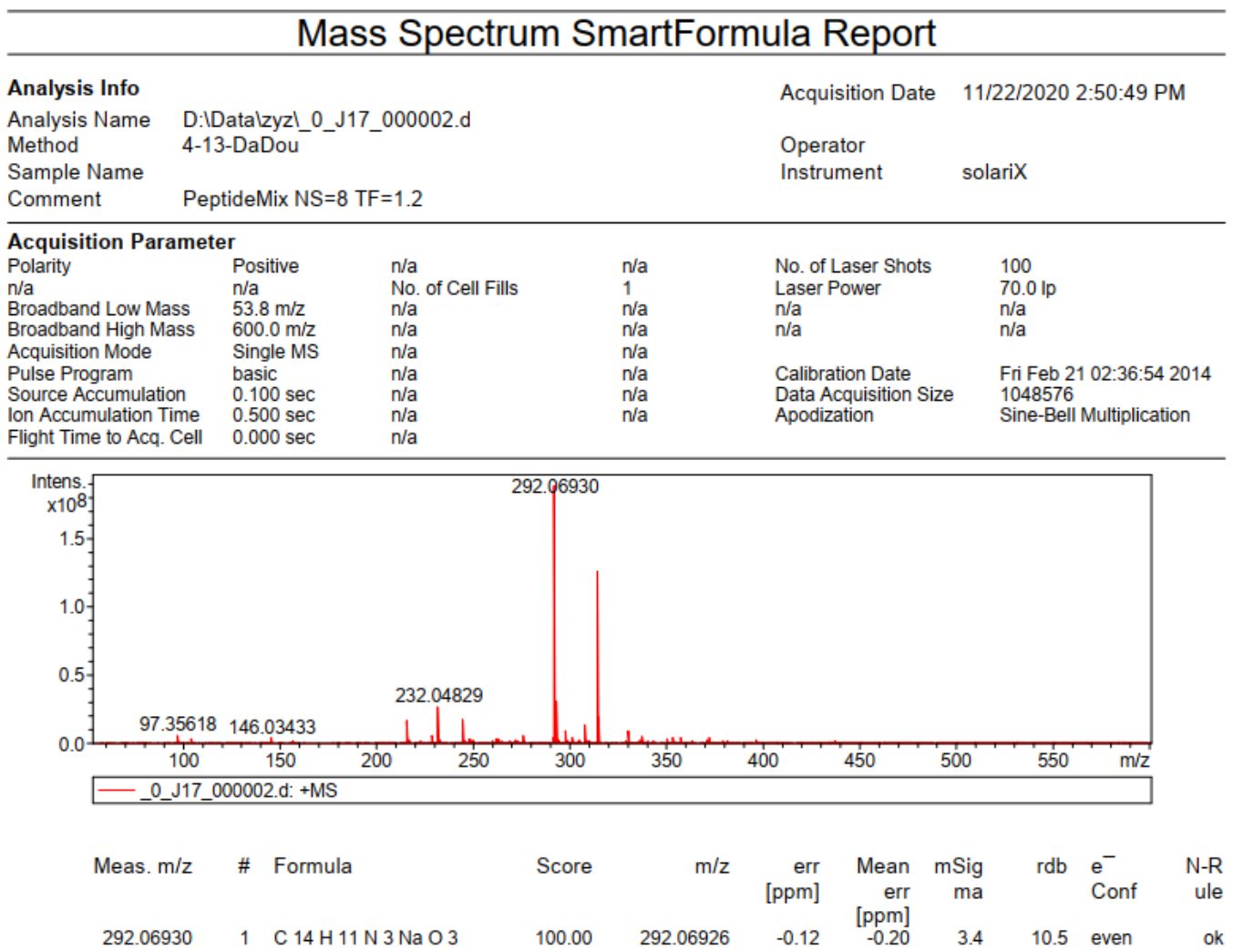


13

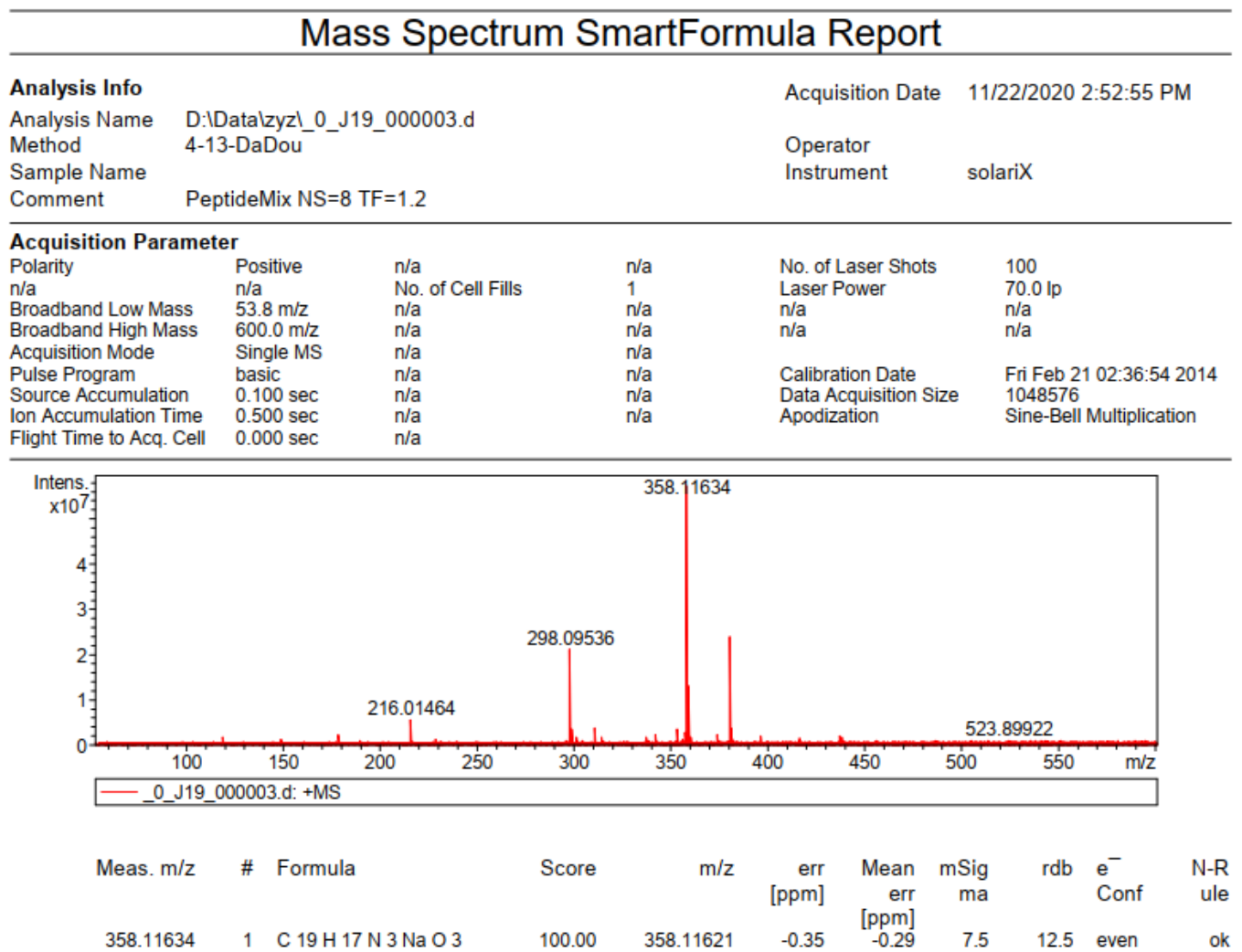

14

$\begin{array}{lllllll}\begin{array}{l}\text { Sample Name } \\ \text { Inj Vol }\end{array} & \mathrm{XJ}-14 & \text { Position } & \mathrm{P} 1-\mathrm{B} 2 & \begin{array}{l}\text { Instrument Name } \\ \text { SampleType }\end{array} & \begin{array}{l}\text { Instrument 1 } \\ \text { Sample }\end{array} & \begin{array}{l}\text { User Name } \\ \text { IRM Calibration Status }\end{array} \\ \text { Data Filename } & \mathrm{XJ}-14 . \mathrm{d} & \begin{array}{l}\text { InjPosition } \\ \text { ACQ Method }\end{array} & \text { JYH.m } & \begin{array}{l}\text { Somment } \\ \text { Acquired Time }\end{array} & & 11 / 24 / 20201: 32: 55 \text { PM }\end{array}$

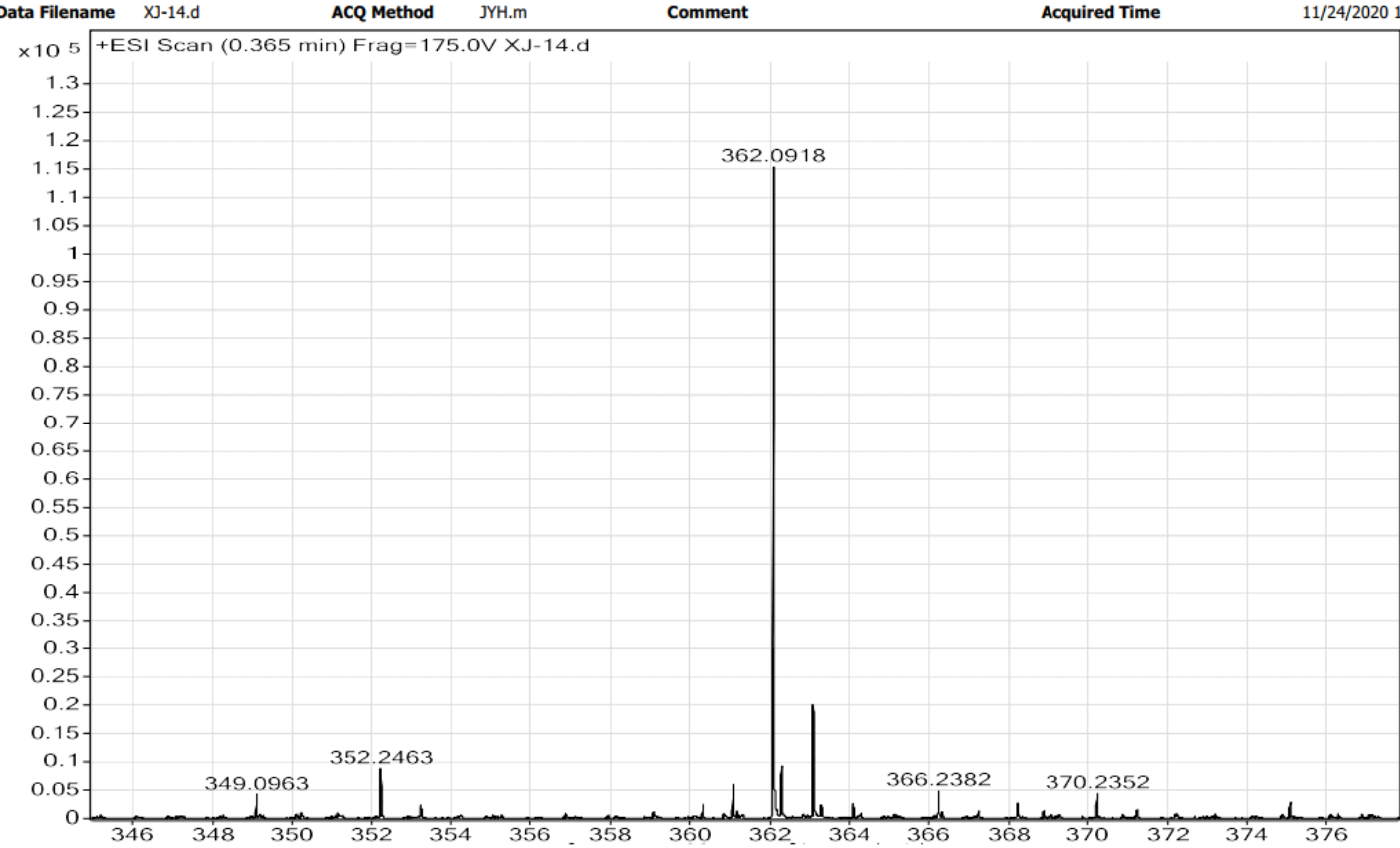

\begin{tabular}{|c|r|r|r|r|r|r|}
\hline Formula (M) & Score (MFG) & \multicolumn{1}{c|}{ Mass } & Mass (MFG) & m/z (Calc) & Diff (ppm) & DBE \\
\hline C18 H14 F N3 O3 & 98.66 & 339.1026 & 339.1019 & 362.0911 & -1.94 & 362.0918 \\
\hline
\end{tabular}




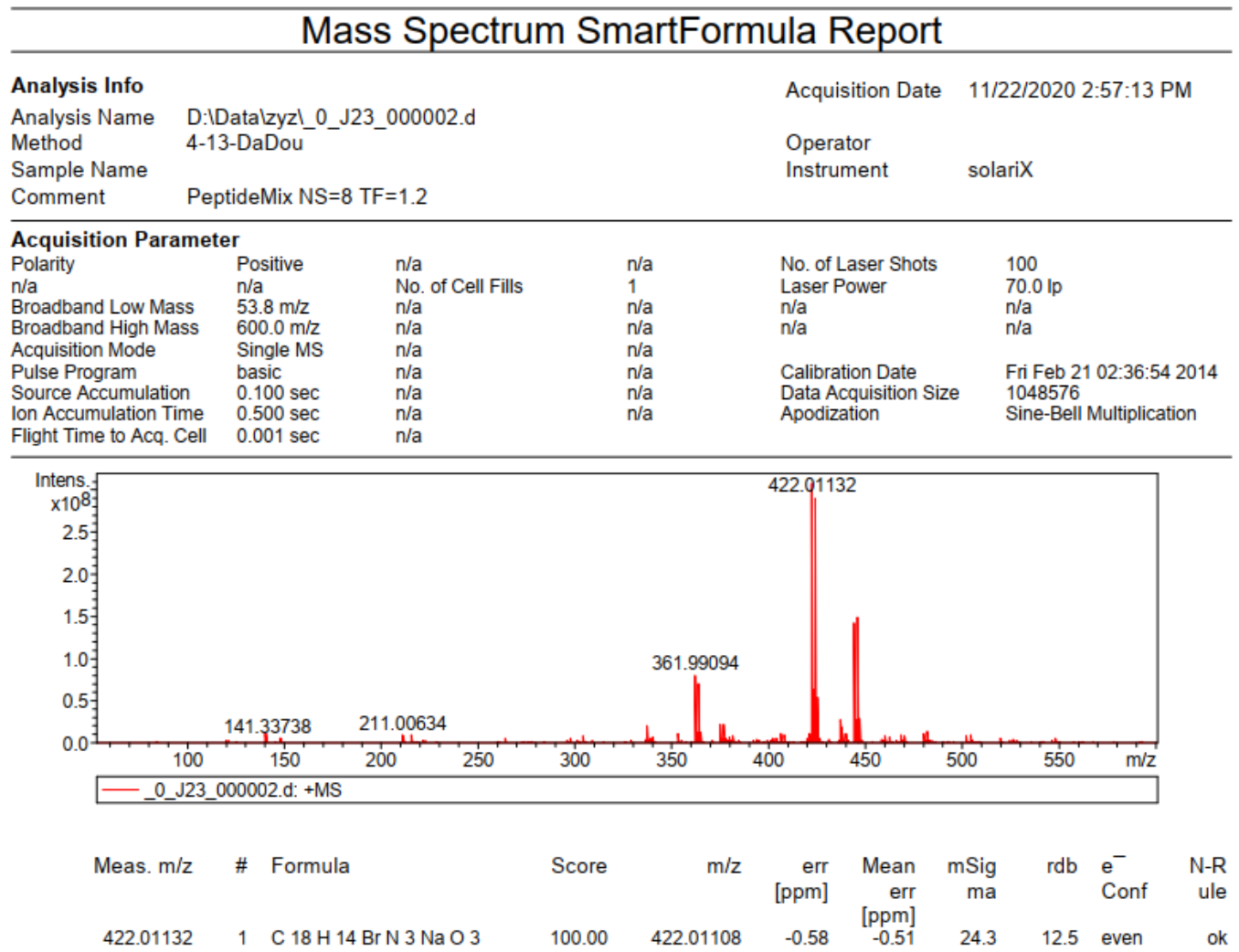

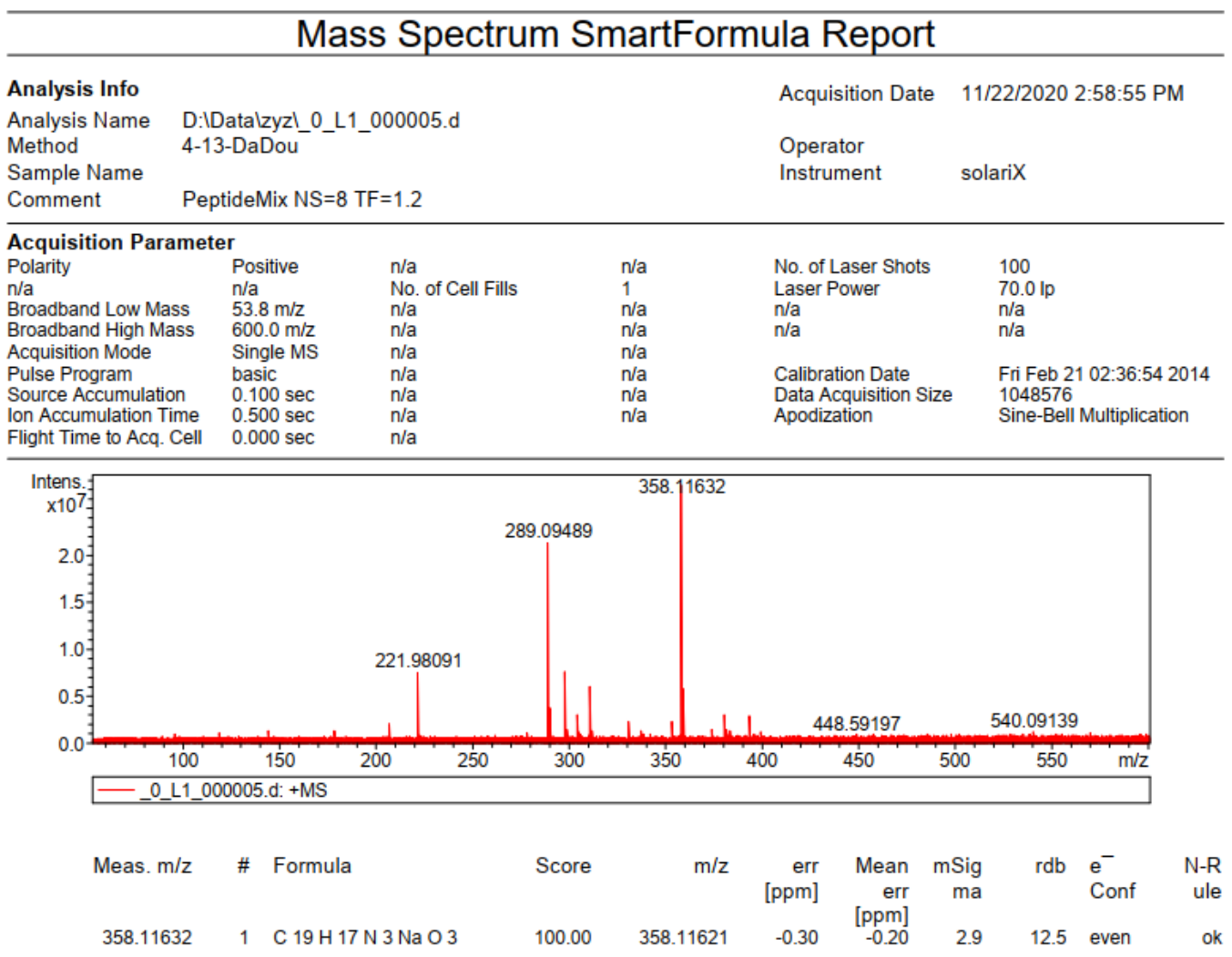




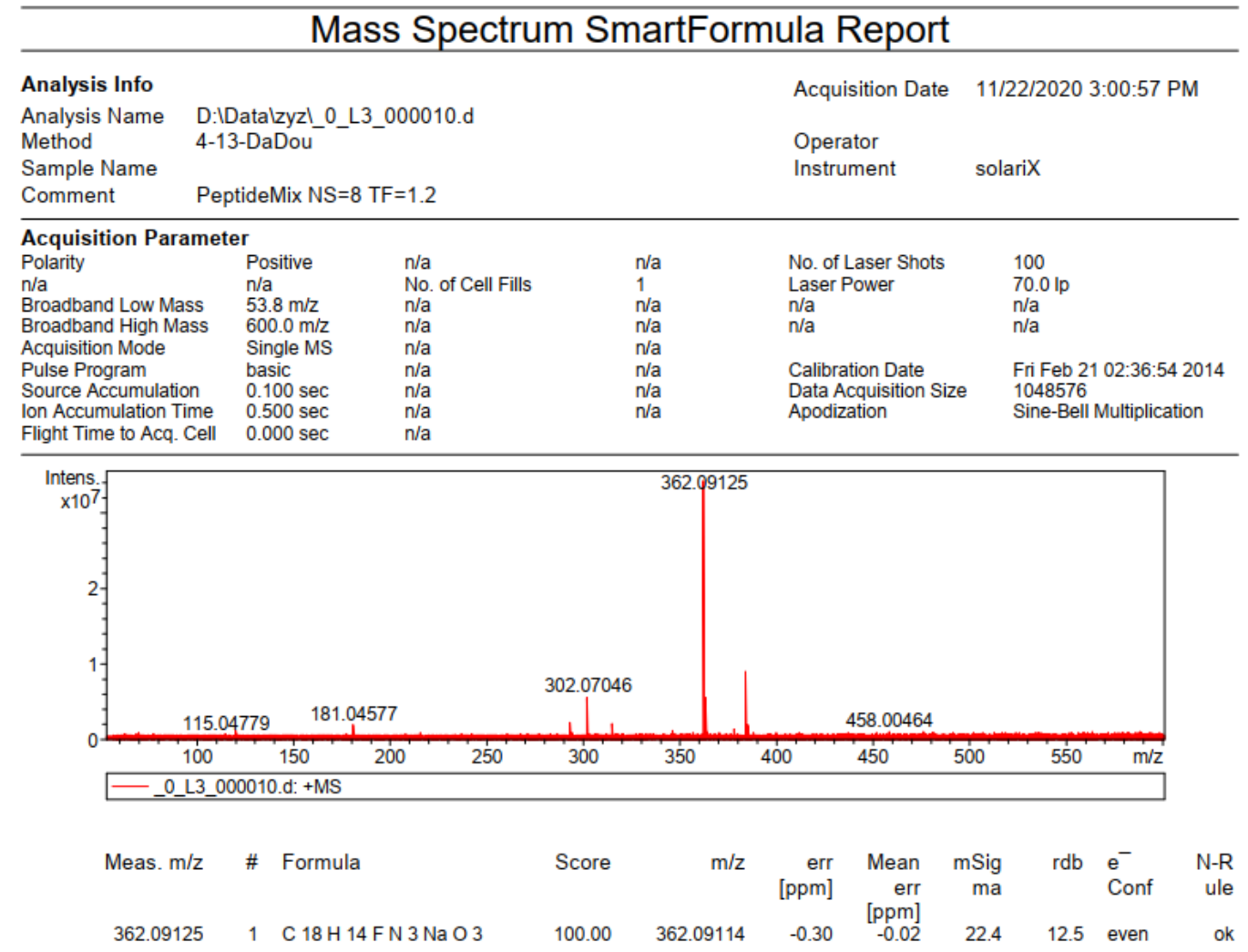

18

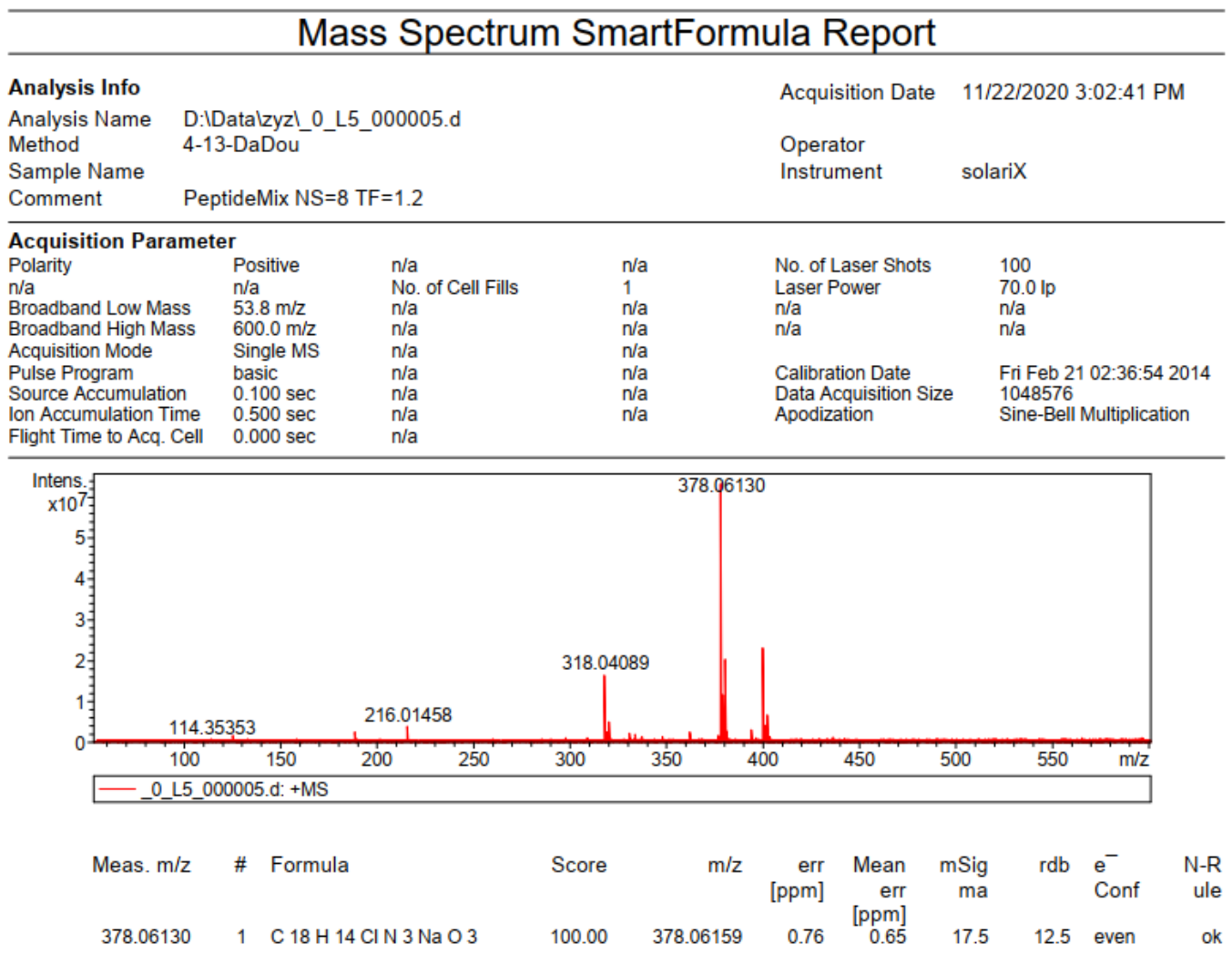


19

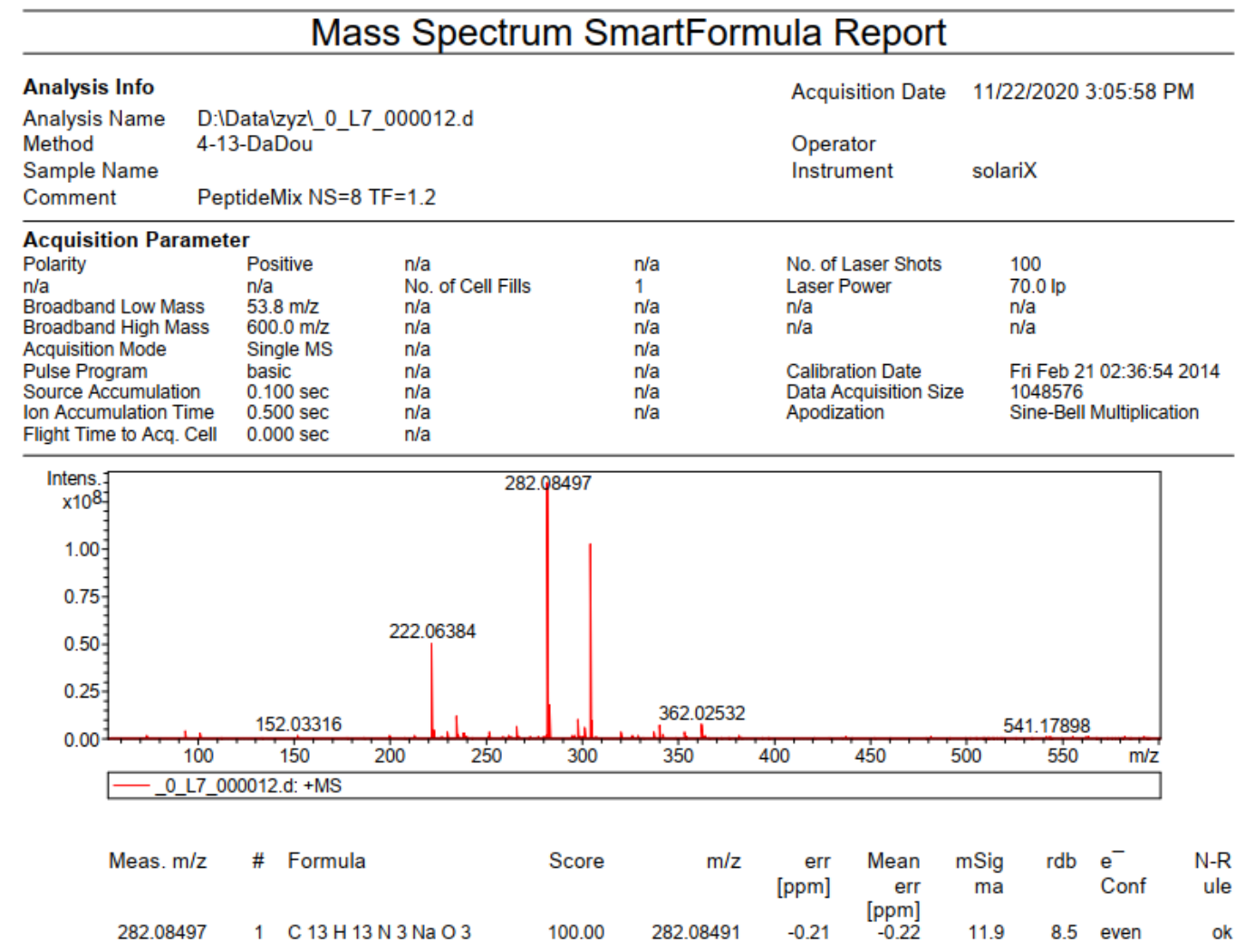

20

\section{Mass Spectrum SmartFormula Report}

Analysis Info

Analysis Name D:IDatal202010617/719_2-F,3_01_13189.d

Method

Ic_pos_3min.m

719

Comment

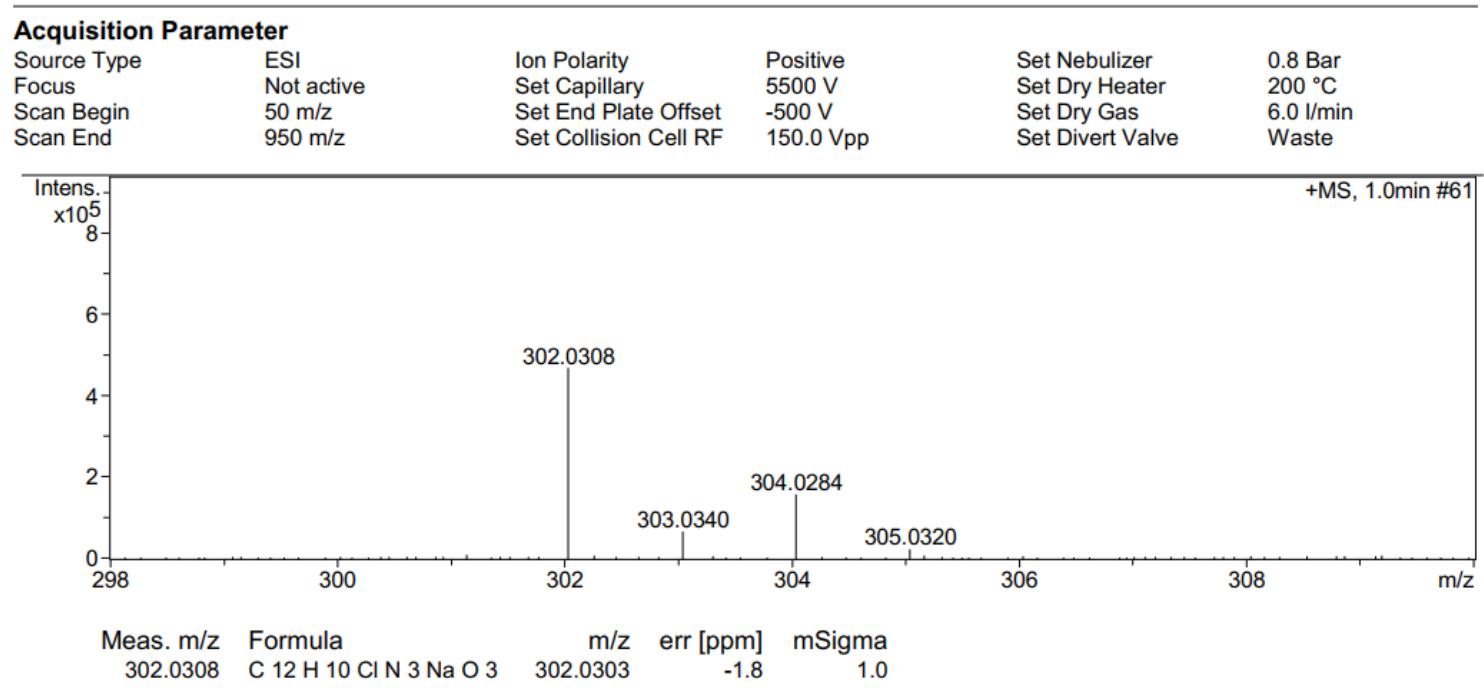

Acquisition Date $\quad 6 / 17 / 2020$ 8:07:37 PM

Operator BDAL@DE

Instrument / Ser\# micrOTOF-Q II 10366 
21

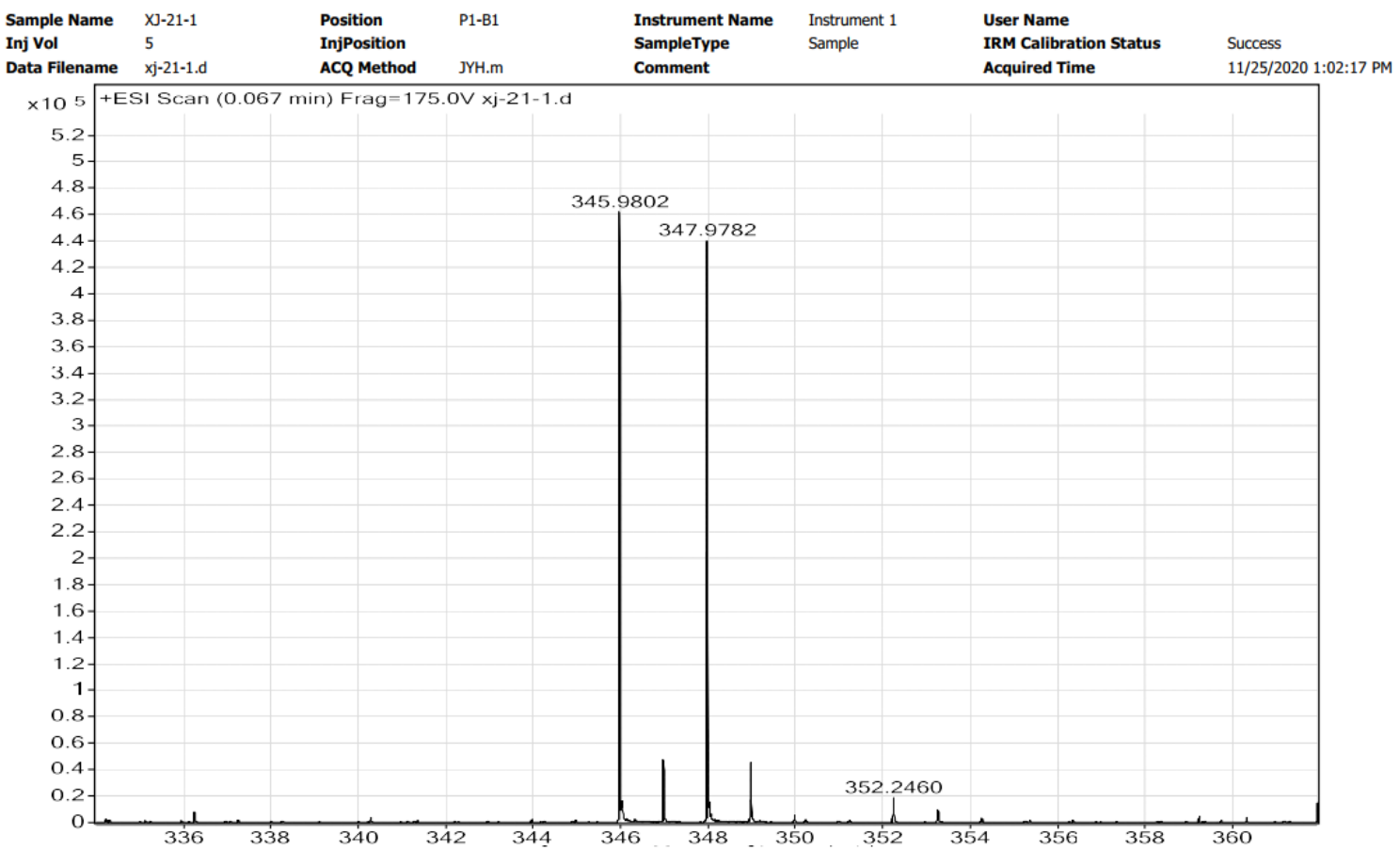

\begin{tabular}{|c|r|r|r|r|r|r|r|}
\hline Formula (M) & Score (MFG) & \multicolumn{1}{c|}{ Mass } & Mass (MFG) & $\mathrm{m} / \mathrm{z}$ (Calc) & Diff (ppm) & DBE & $\mathrm{m} / \mathrm{z}$ \\
\hline $\mathrm{C} 12 \mathrm{H} 10 \mathrm{Br} \mathrm{N} 3 \mathrm{O} 3$ & 99.41 & 322.991 & 322.9906 & 345.9798 & -1.32 & 9 & 345.9802 \\
\hline
\end{tabular}

22

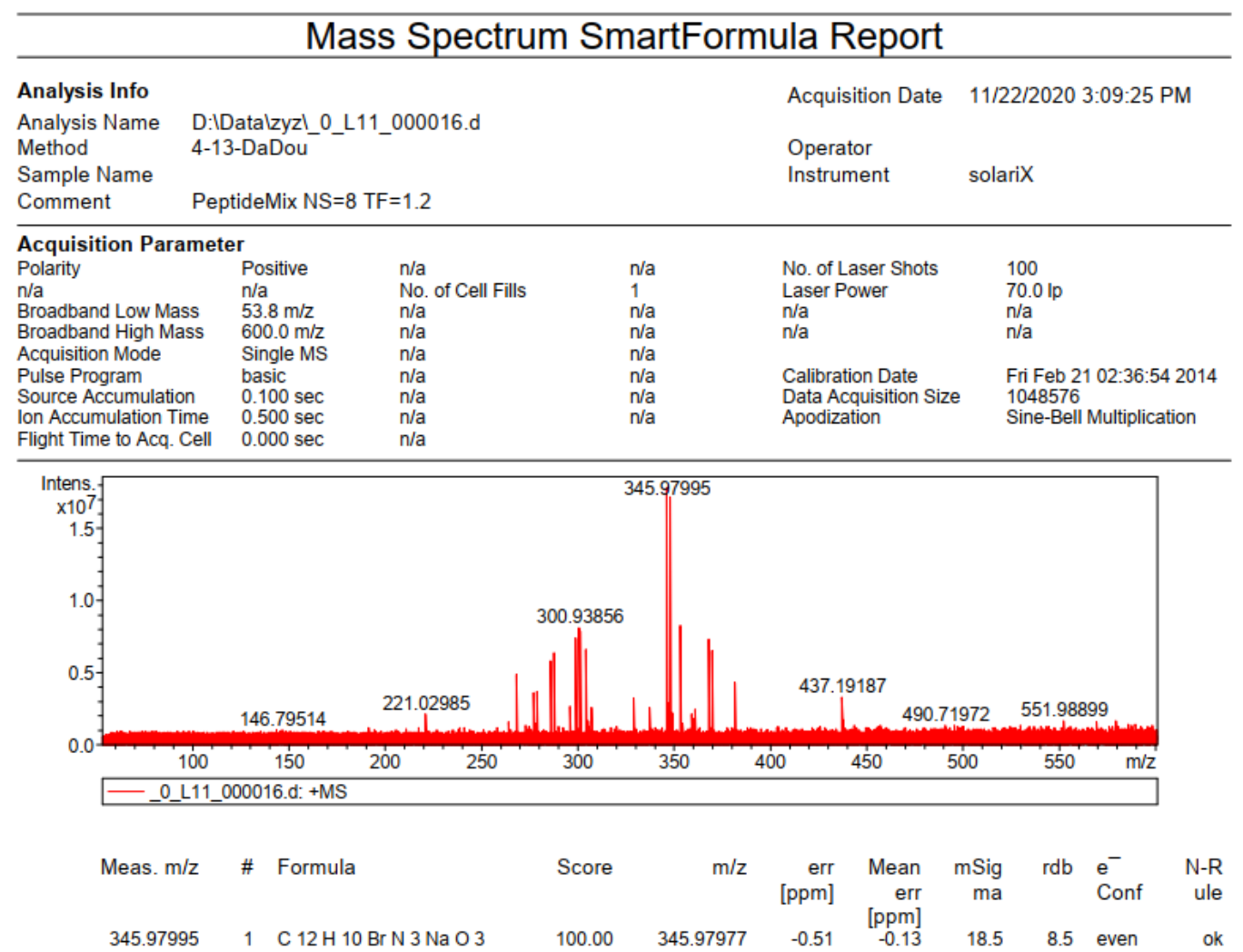




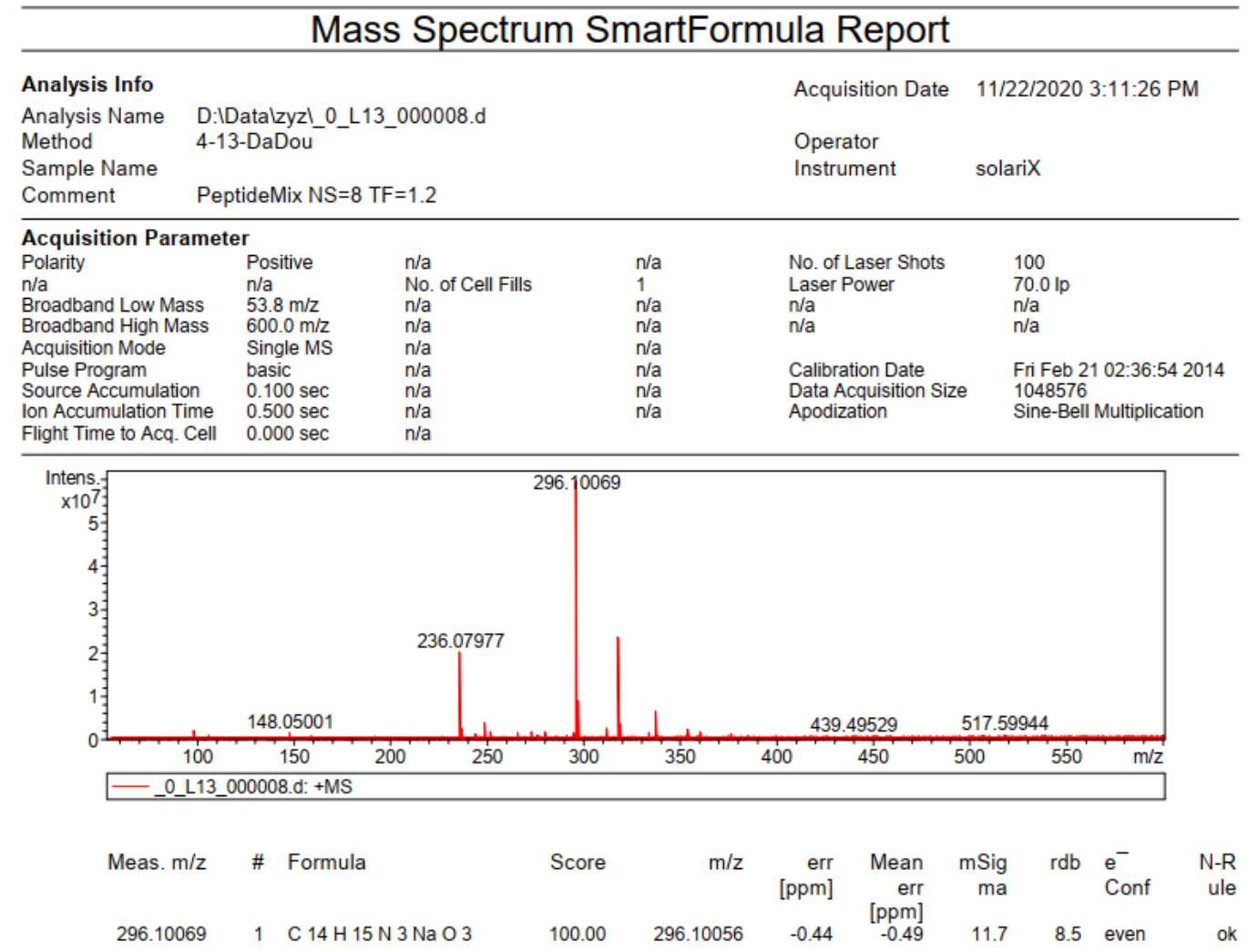

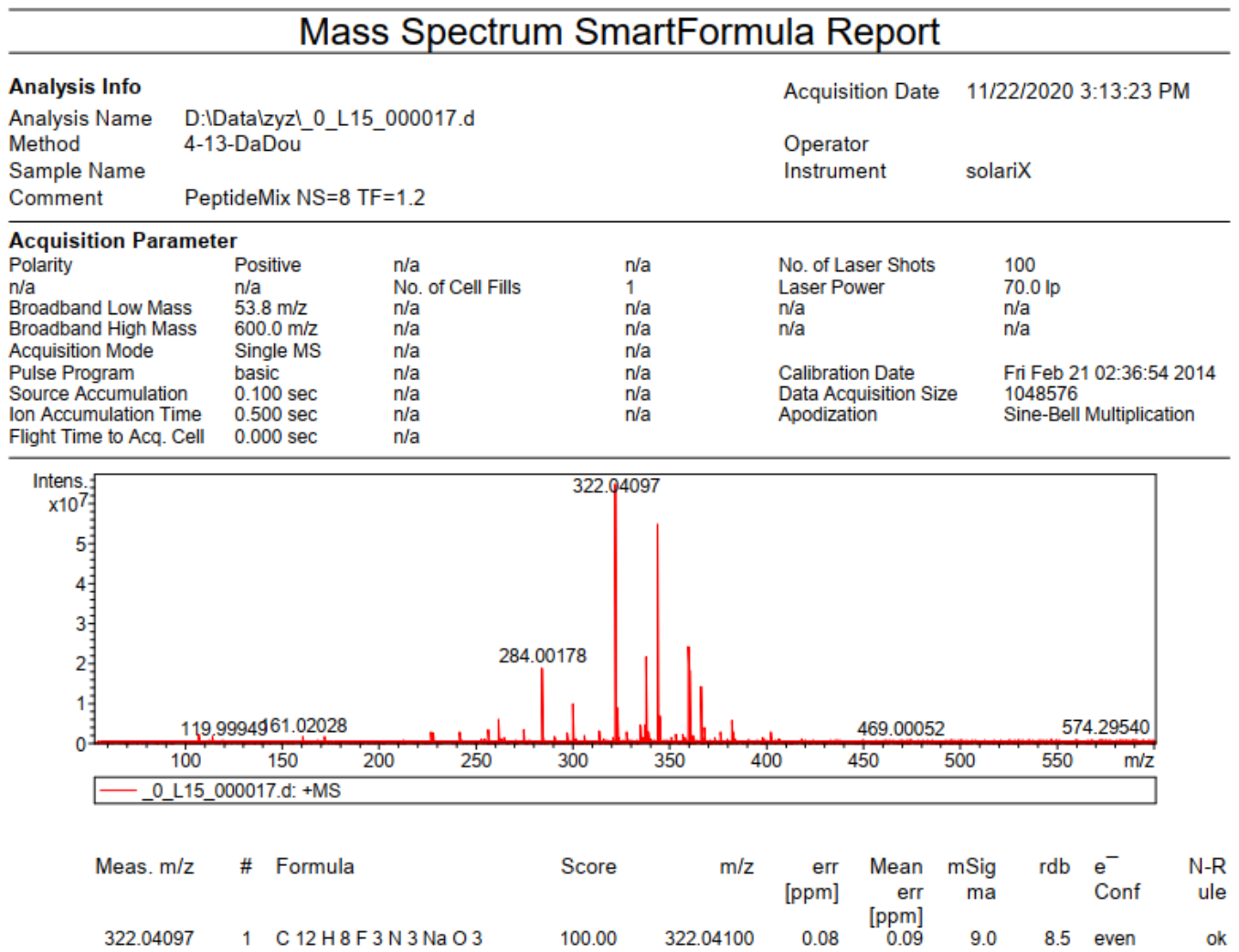


25

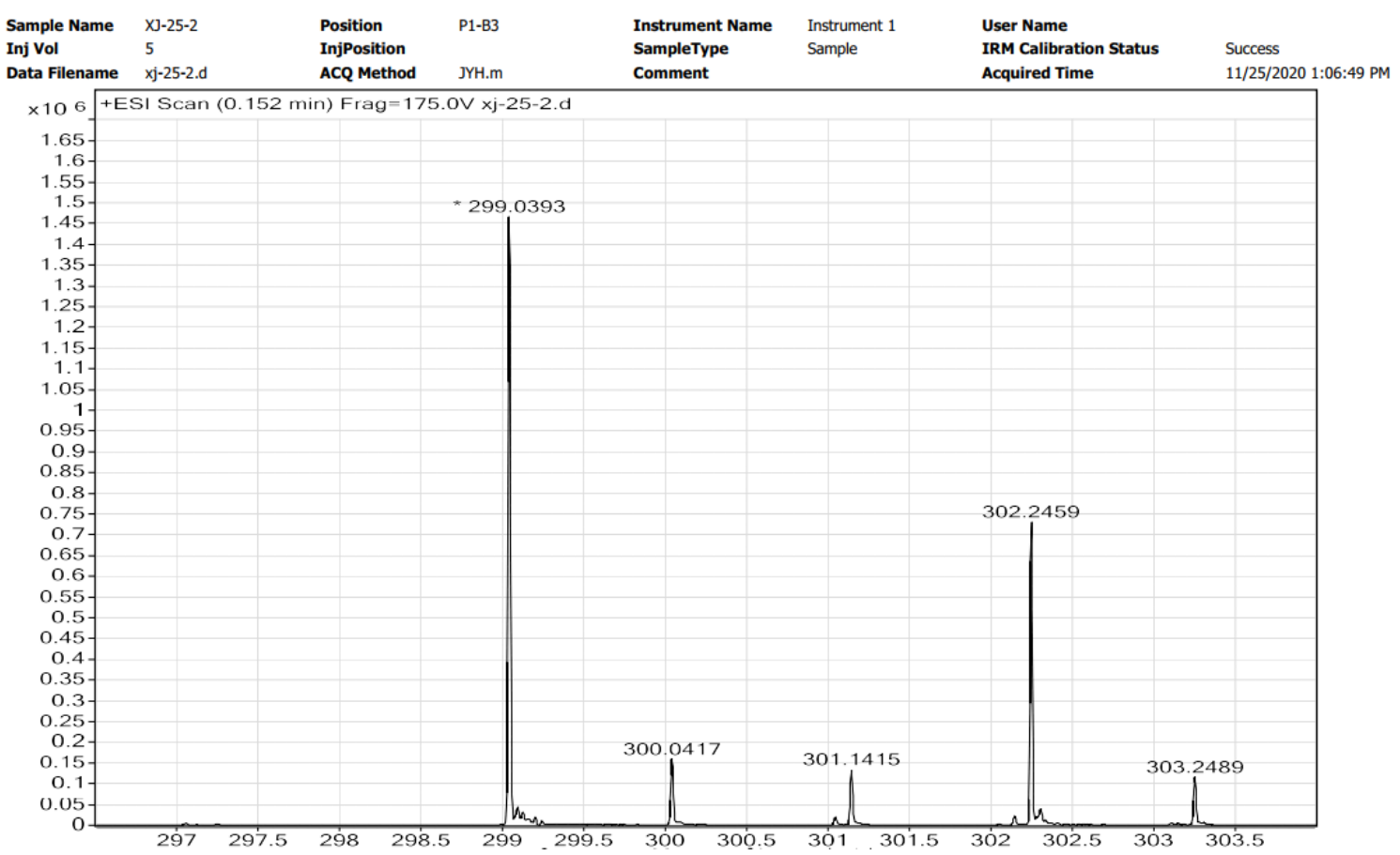

\begin{tabular}{|c|r|r|r|r|r|r|r|}
\hline Formula (M) & Score (MFG) & \multicolumn{1}{c|}{ Mass } & Mass (MFG) & m/z (Calc) & Diff (ppm) & DBE & $\mathrm{m} / \mathrm{z}$ \\
\hline C11 H8 N4 O5 & 98.61 & 276.0501 & 276.0495 & 299.0387 & -2.21 & 10 & 299.0393 \\
\hline
\end{tabular}

26

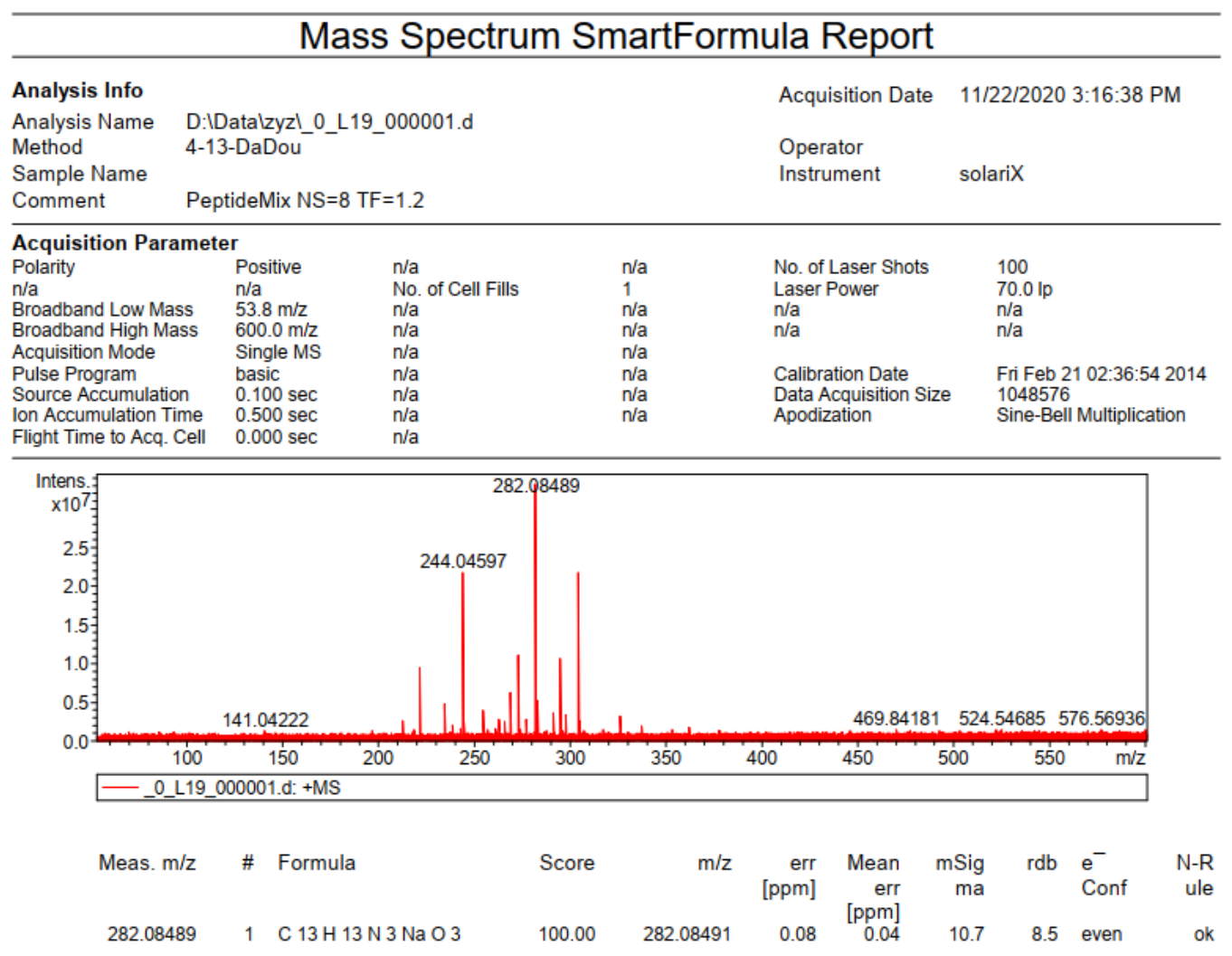




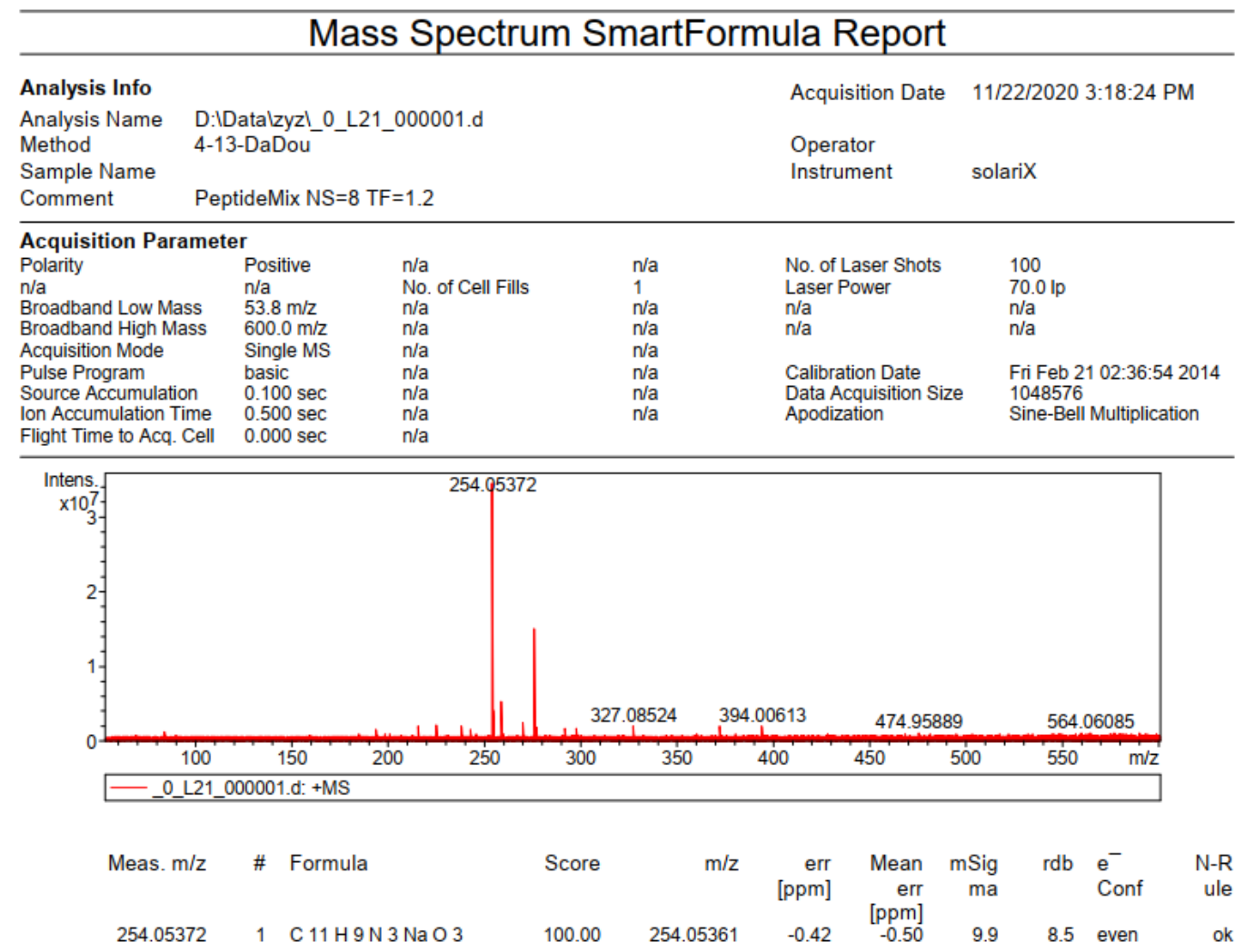

28

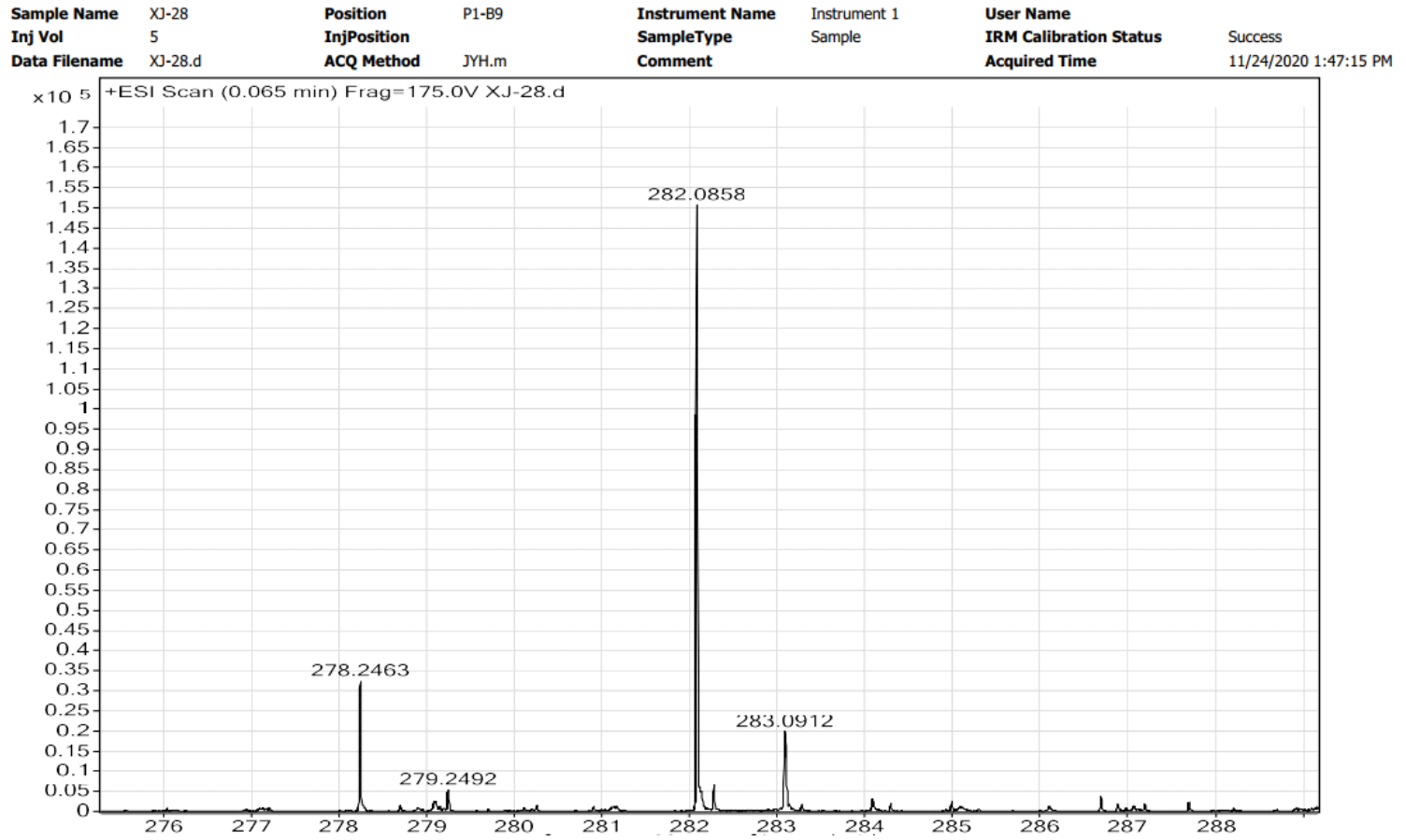

\begin{tabular}{|l|r|r|r|r|r|r|r|}
\hline Formula (M) & Score (MFG) & \multicolumn{1}{c|}{ Mass } & Mass (MFG) & $\mathrm{m} / \mathrm{z}($ Calc) & Diff (ppm) & DBE & $\mathrm{m} / \mathrm{z}$ \\
\hline C13 H13 N3 O3 & 96.95 & 259.0966 & 259.0957 & 282.0849 & -3.43 & 9 & 282.0858 \\
\hline
\end{tabular}




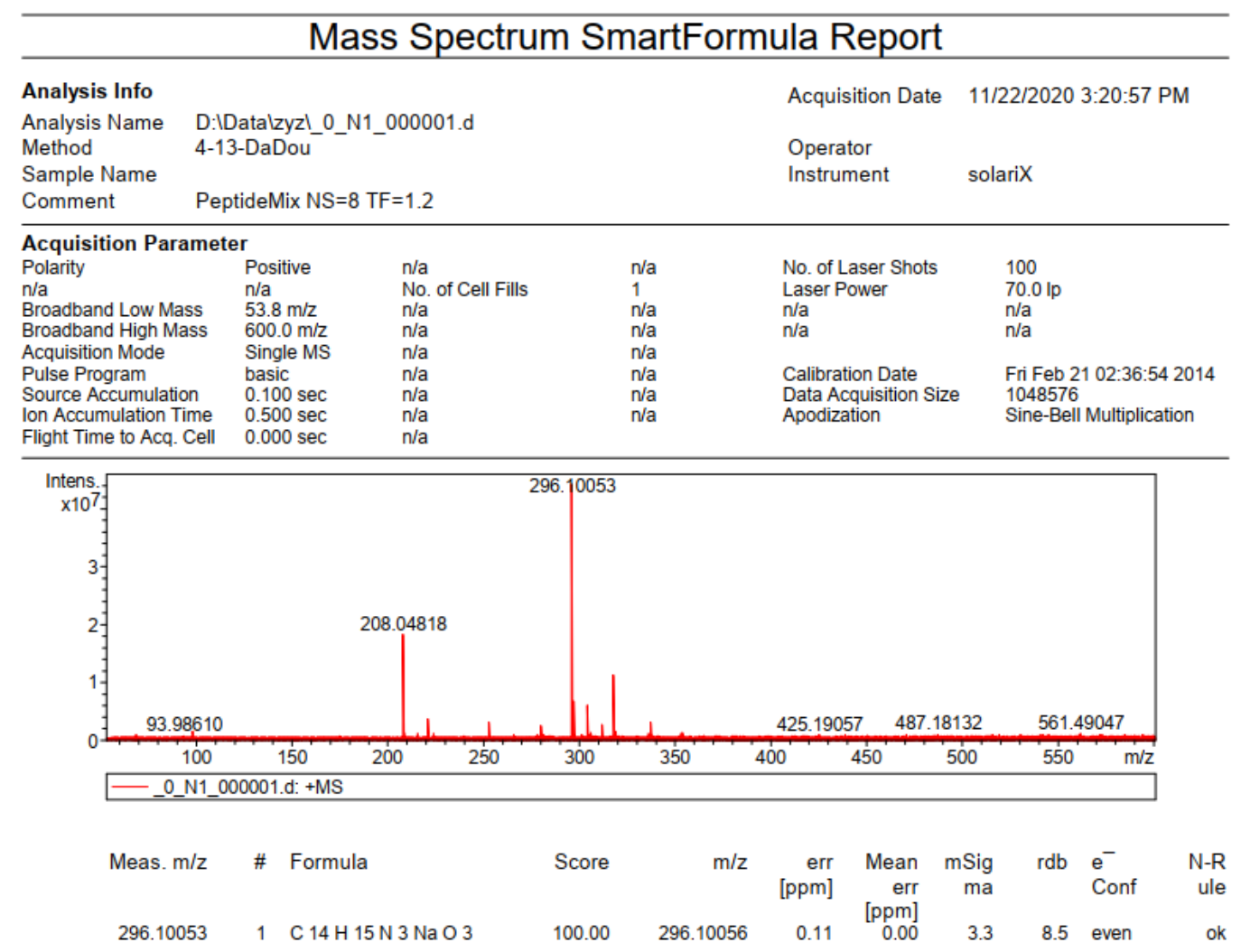

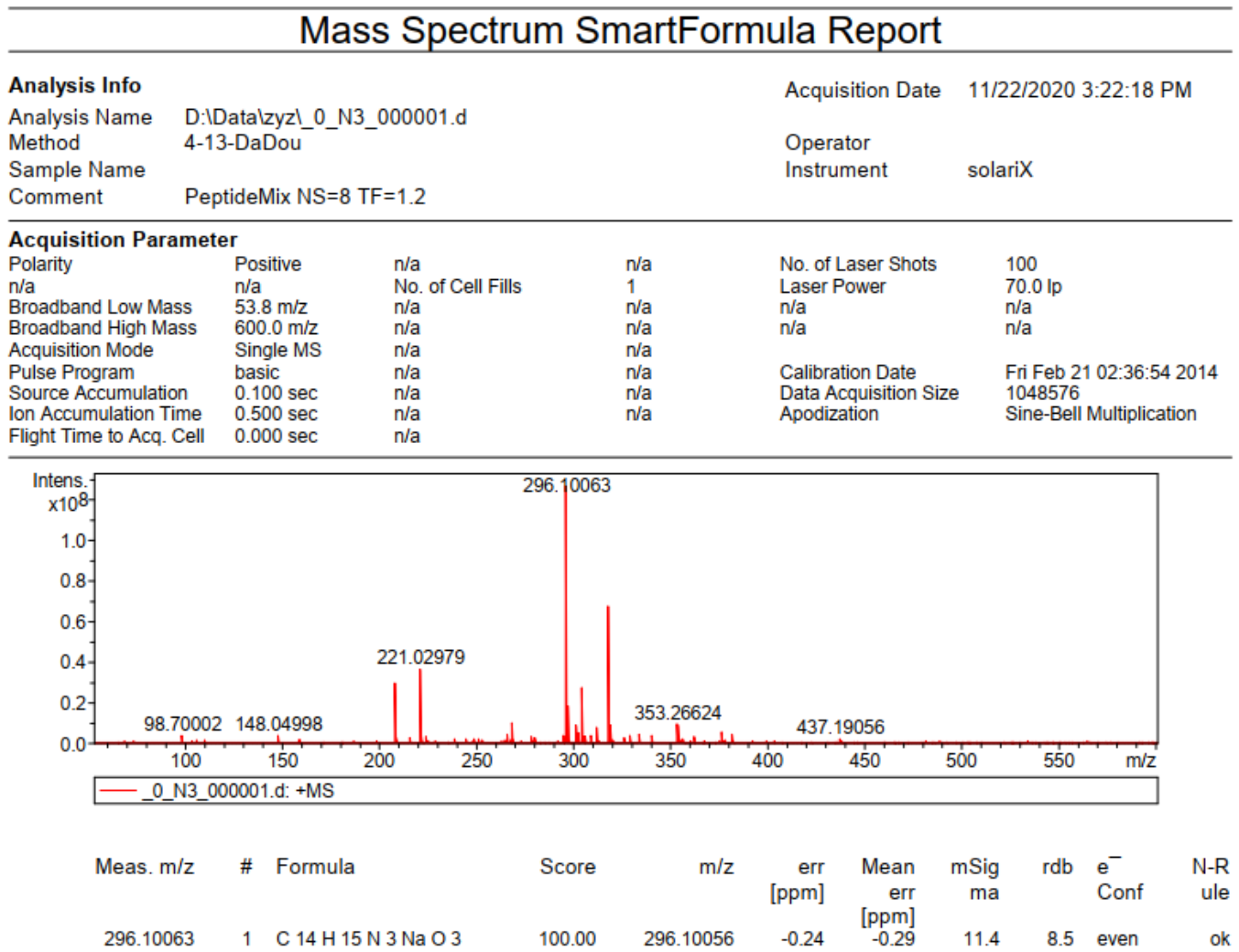




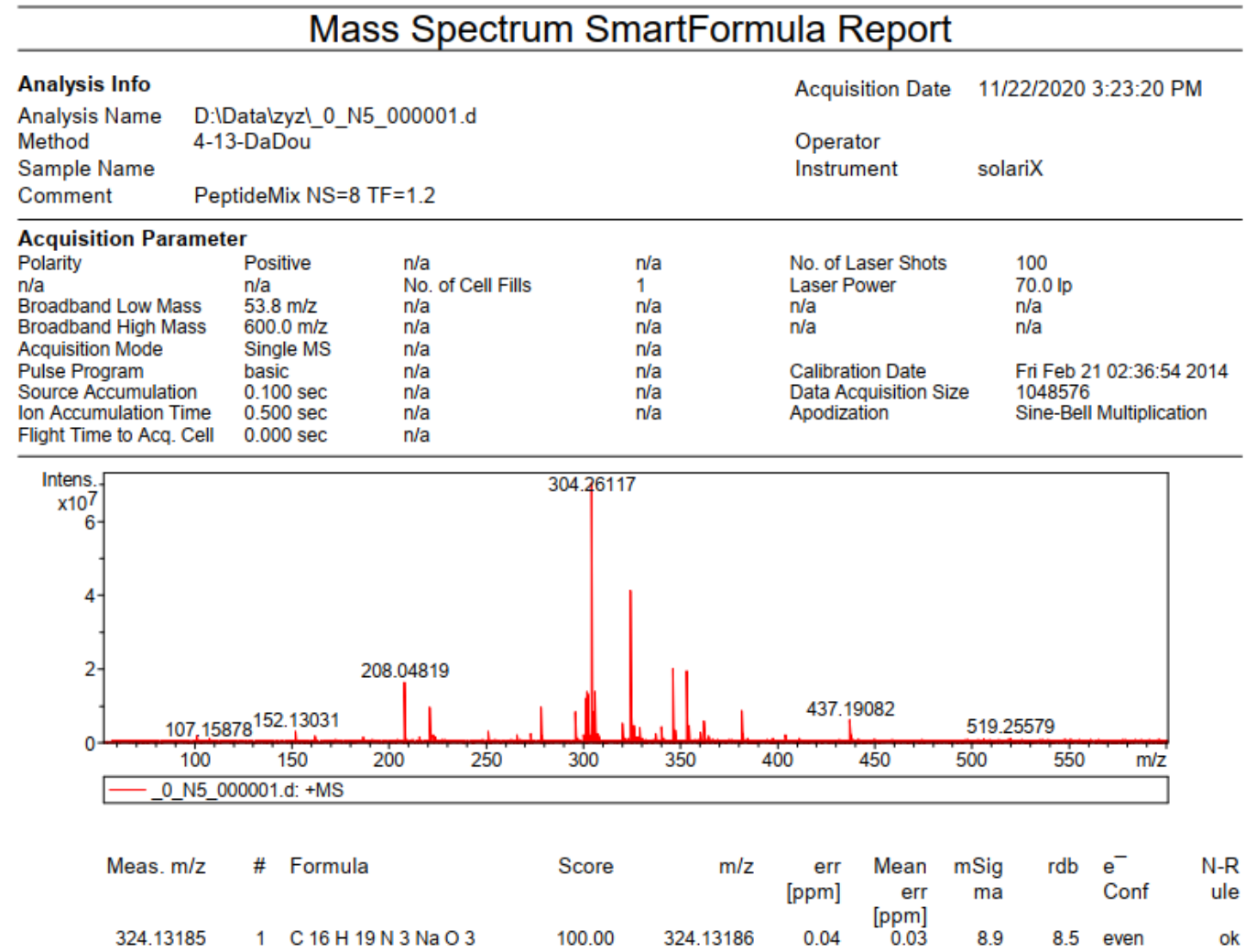

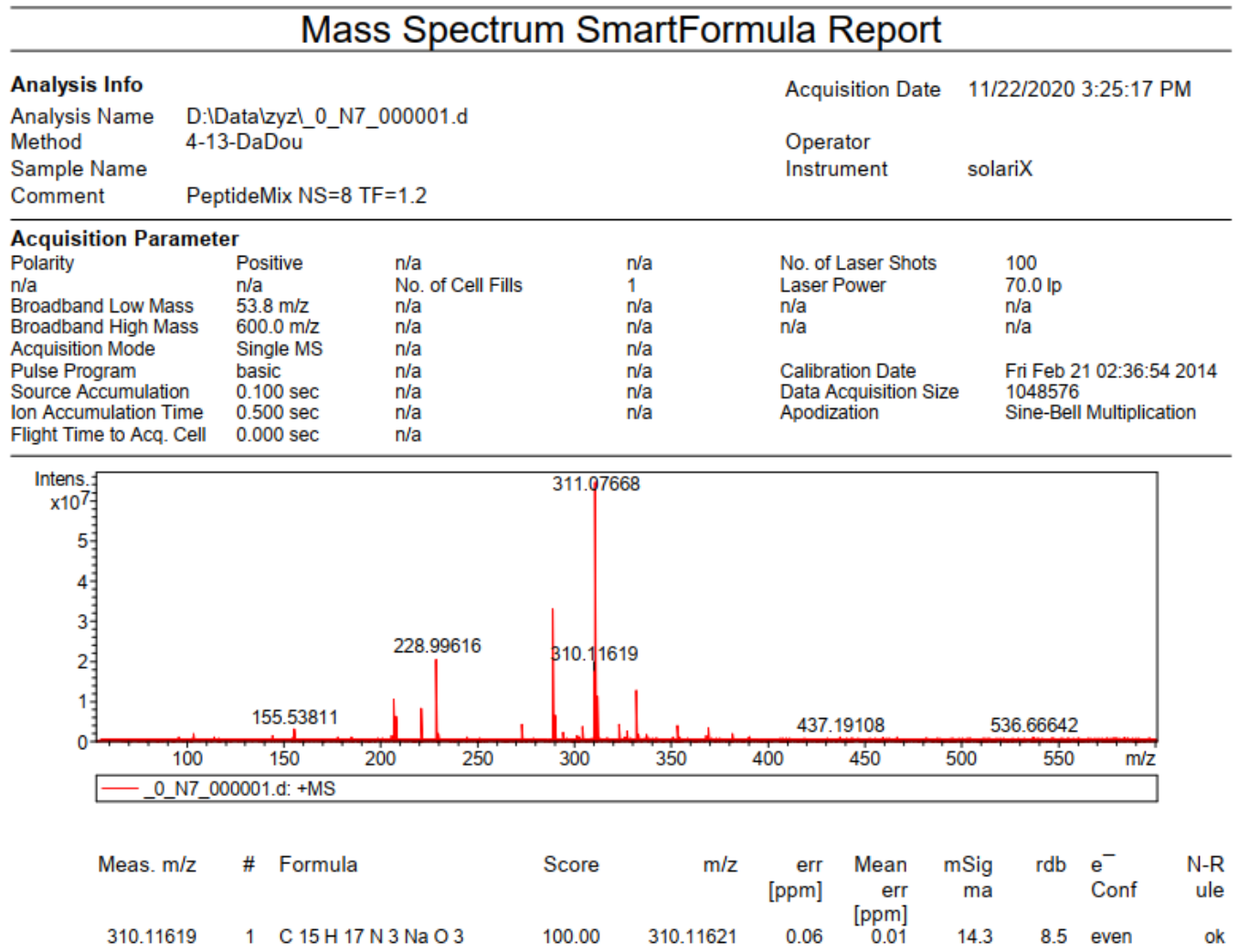




\section{Mass Spectrum SmartFormula Report}

Analysis Info

Analysis Name D:IDatalzyzl_0_N9_000001.d

Method

Sample Name

Comment
4-13-DaDou

PeptideMix NS=8 TF=1.2
Acquisition Date 11/22/2020 3:27:58 PM

Operator

Instrument solariX

\section{Acquisition Parameter}

Polarity

Broadband Low Mass Broadband High Mass

Acquisition Mode

Pulse Program

Source Accumulation

Positive

n/a $53.8 \mathrm{~m} / \mathrm{z}$

$53.8 \mathrm{~m} / \mathrm{z}$
$600.0 \mathrm{~m} / \mathrm{z}$

$600.0 \mathrm{~m} / \mathrm{z}$
Single MS

basic

$0.100 \mathrm{sec}$

Flight Time to Acq. Cell $\quad 0.000 \mathrm{sec}$

$\begin{array}{llll}\text { n/a } & \text { n/a } & \text { No. of Laser Shots } & 100 \\ \text { No. of Cell Fills } & 1 & \text { Laser Power } & 70.0 \mathrm{Ip} \\ \text { n/a } & \text { n/a } & \text { n/a } & \text { n/a } \\ \text { n/a } & \text { n/a } & \text { n/a } & \text { n/a } \\ \text { n/a } & \text { n/a } & \text { Calibration Date } & \text { Fri Feb 21 02:36:54 2014 } \\ \text { n/a } & \text { n/a } & \text { Data Acquisition Size } & 1048576 \\ \text { n/a } & \text { n/a } & \text { Apodization } & \text { Sine-Bell Multiplication } \\ \text { n/a } & \text { n/a } & & \end{array}$

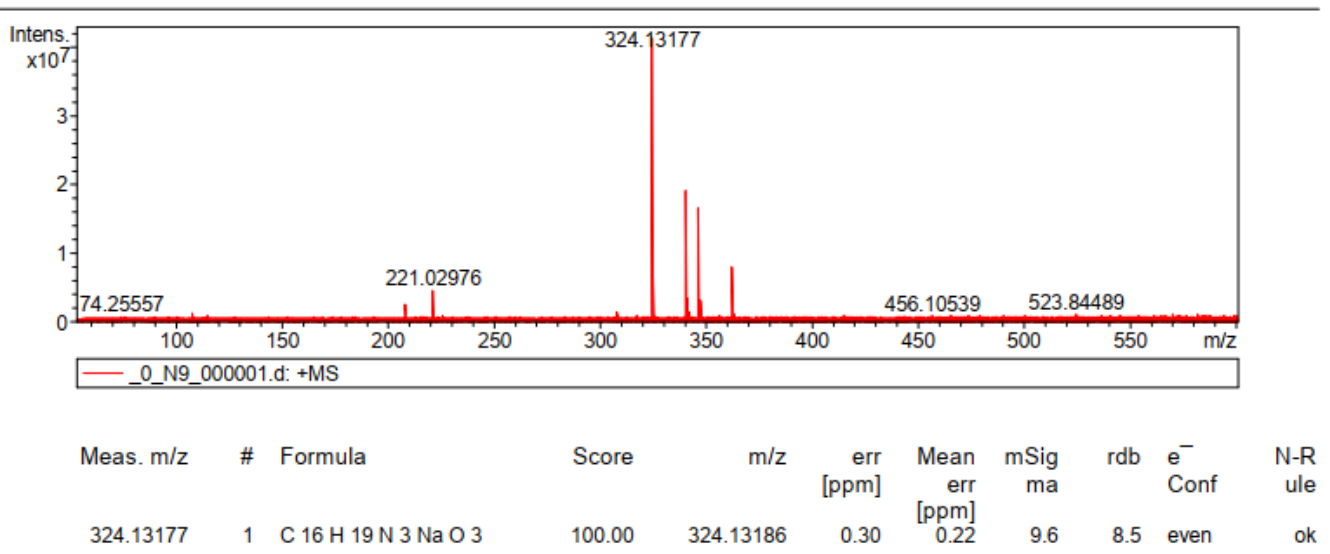

34

\section{Mass Spectrum SmartFormula Report}

Analysis Info

Analysis Name D:IDatalzyzI_0_N11_000002.d

Method

Sample Name

Comment
PeptideMix NS=8 TF=1.2
Acquisition Date 11/22/2020 3:29:18 PM

Operator

Instrument solariX

\section{Acquisition Parameter}

Polarity

n/a
Broadband Low Mass

Broadband High Mass

Acquisition Mode

Pulse Program

n/a

$53.8 \mathrm{~m} / \mathrm{z}$

$600.0 \mathrm{~m} / \mathrm{z}$

Single MS

basic

$0.100 \mathrm{sec}$

$0.500 \mathrm{sec}$

$0.000 \mathrm{sec}$

Flight Time to Acq. Cell

$\begin{array}{ll}\mathrm{n} / \mathrm{a} & \mathrm{n} \\ \mathrm{No} . \text { of Cell Fills } & 1 \\ \mathrm{n} / \mathrm{a} & \mathrm{n} \\ \mathrm{n} / \mathrm{a} & \mathrm{n} \\ \mathrm{n} / \mathrm{a} & \mathrm{n} \\ \mathrm{n} / \mathrm{a} & \mathrm{n} / \mathrm{a} \\ \mathrm{n} / \mathrm{a} & \mathrm{n} / \mathrm{a} \\ \mathrm{n} / \mathrm{a} & \mathrm{n} / \mathrm{a}\end{array}$

No. of Laser Shots
Laser Power
n/a
n/a
Calibration Date
Data Acquisition Size
Apodization

100

$70.0 \mathrm{lp}$

n/a

Fri Feb 21 02:36:54 2014

Sine-Bell Multiplication

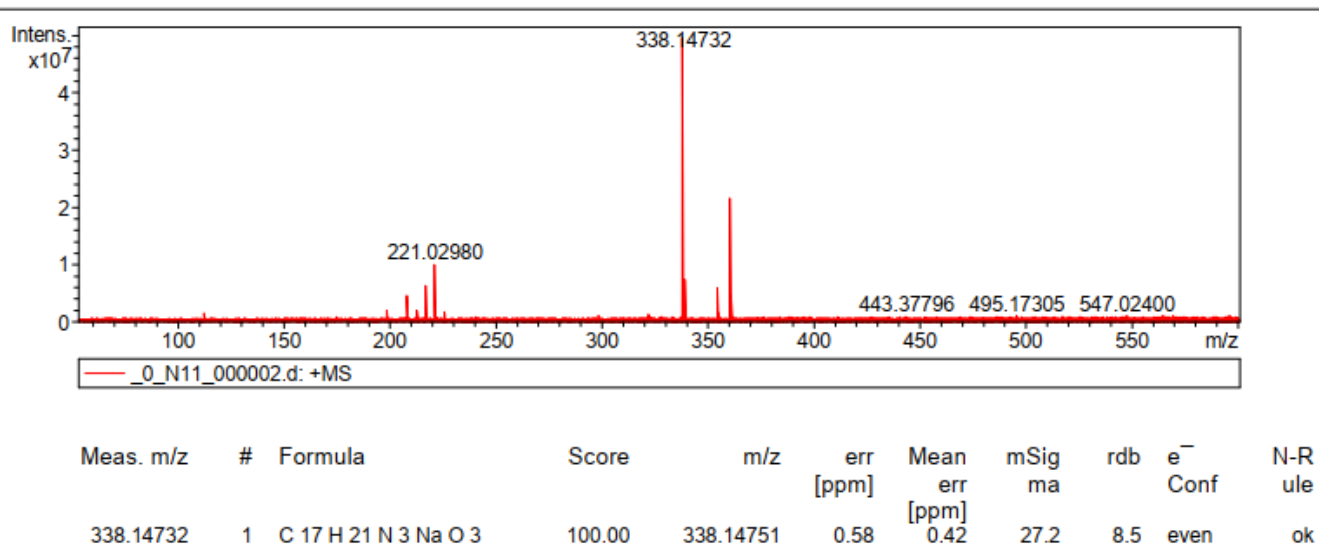




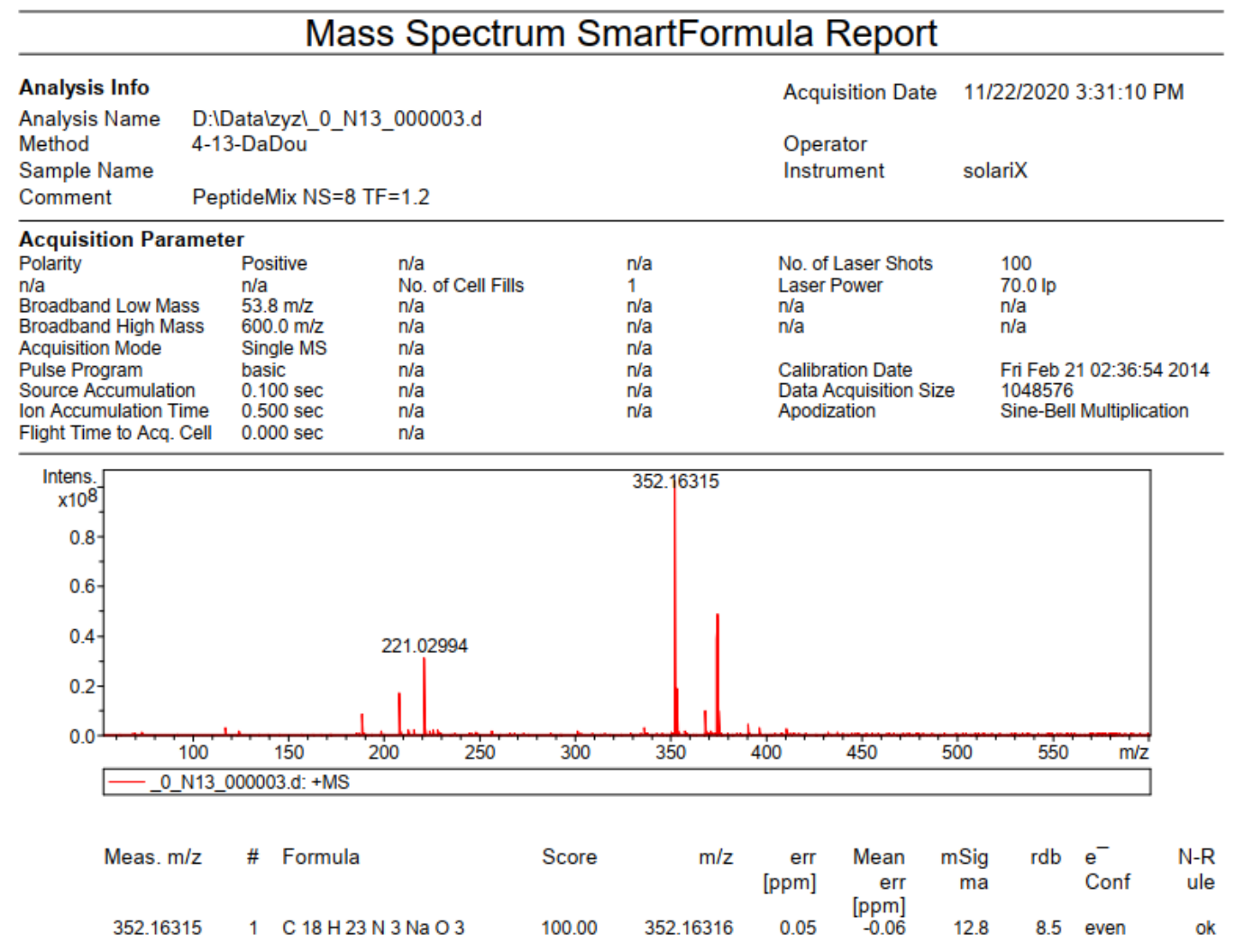

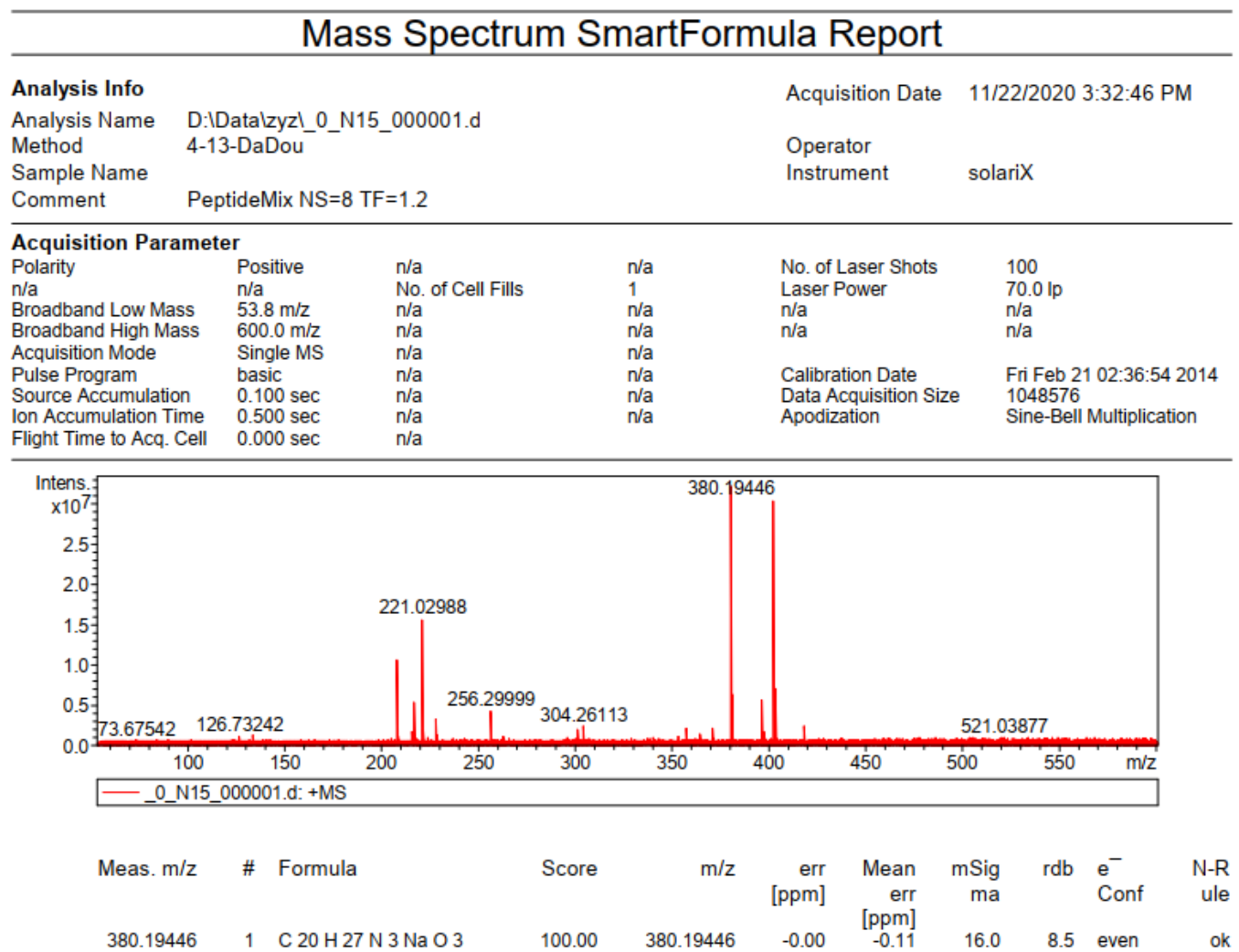




\section{Mass Spectrum SmartFormula Report}

Analysis Info

Analysis Name

Method

Sample Name

Comment

\section{Acquisition Paramete}

Polarity

Broadband Low Mass

Broadband High Mass

Acquisition Mode

Pulse Program

Source Accumulation

Flight Time to Acq. Cell

:IDatalzyzl_0_N17_000003.d

-13-DaDou

PeptideMix NS=8 TF=1.2
Acquisition Date 11/22/2020 3:35:44 PM

Operator

Instrument solariX

No. of Laser Shots $\quad 100$

Laser Power $\quad 70.0 \mathrm{lp}$

$\mathrm{n} / \mathrm{a}$

Calibration Date _ Fri Feb 21 02:36:54 2014 Data Acquisition Size

$\begin{array}{ll}\text { Data Acquisition Size } & 1048576 \\ \text { Apodization } & \text { Sine-Bell Multiplication }\end{array}$

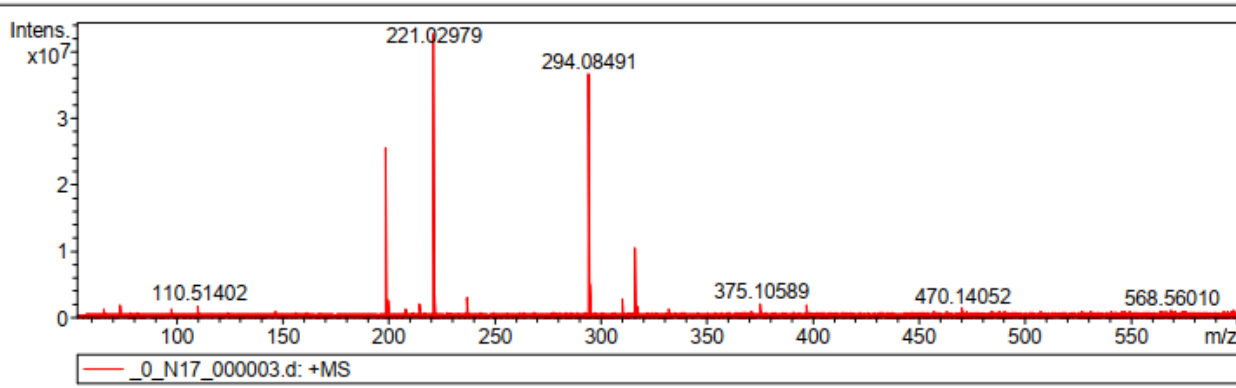

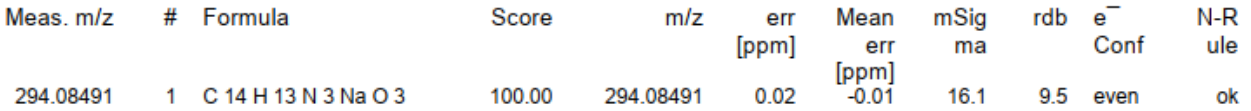

38

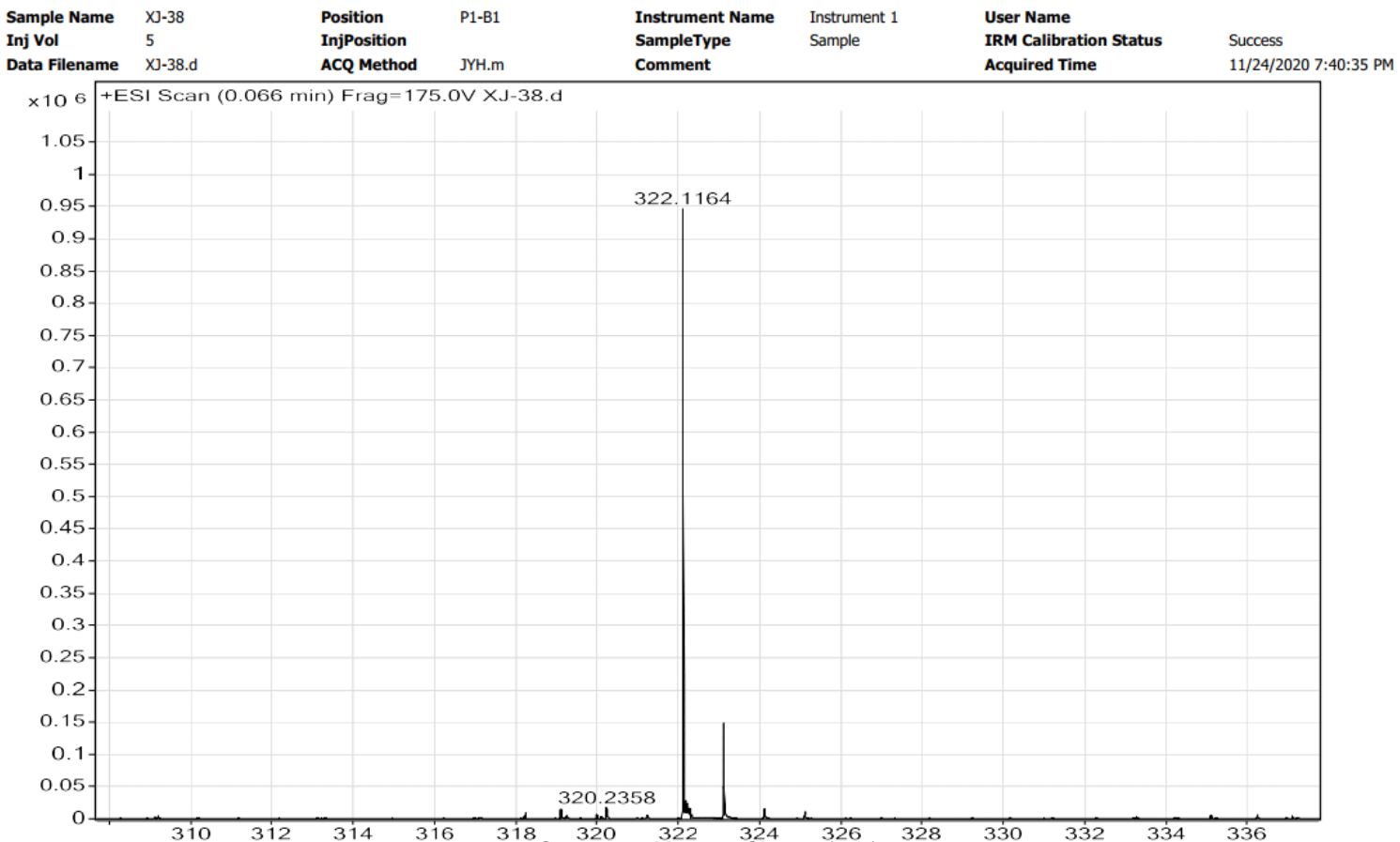

\begin{tabular}{|l|r|r|r|r|r|r|}
\hline Formula (M) & Score (MFG) & Mass & Mass (MFG) & m/z (Calc) & Diff (ppm) & DBE \\
\hline C16 H17 N3 O3 & 99.88 & 299.1272 & 299.127 & 322.1162 & -0.63 & 322.1164 \\
\hline
\end{tabular}


39

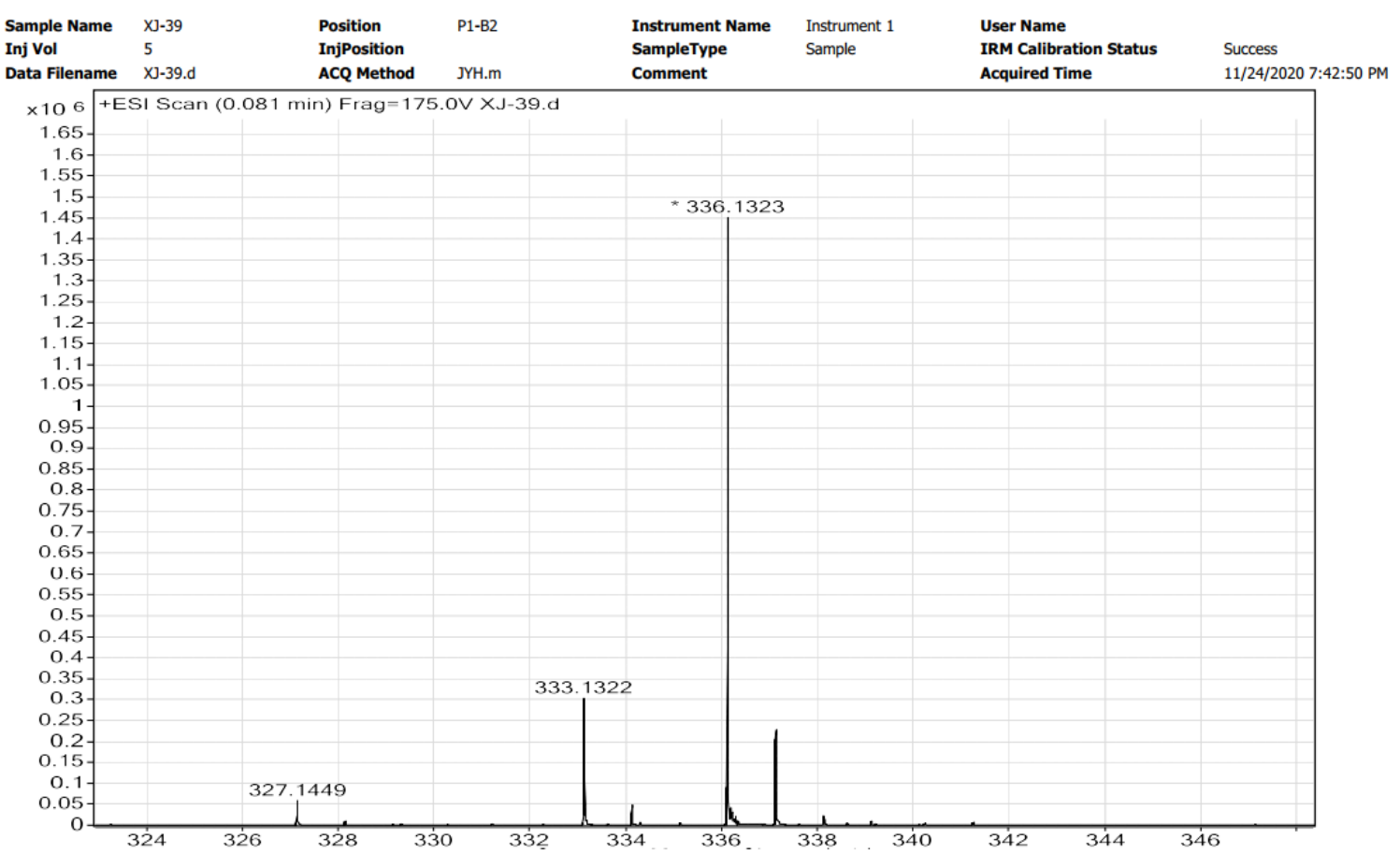

\begin{tabular}{|l|r|r|r|r|r|r|r|}
\hline Formula (M) & Score (MFG) & Mass & Mass (MFG) & m/z (Calc) & Diff (ppm) & DBE & $\mathrm{m} / \mathrm{z}$ \\
\hline C17 H19 N3 O3 & 99.36 & 313.1431 & 313.1426 & 336.1319 & -1.4 & 10 & 336.1323 \\
\hline
\end{tabular}

40

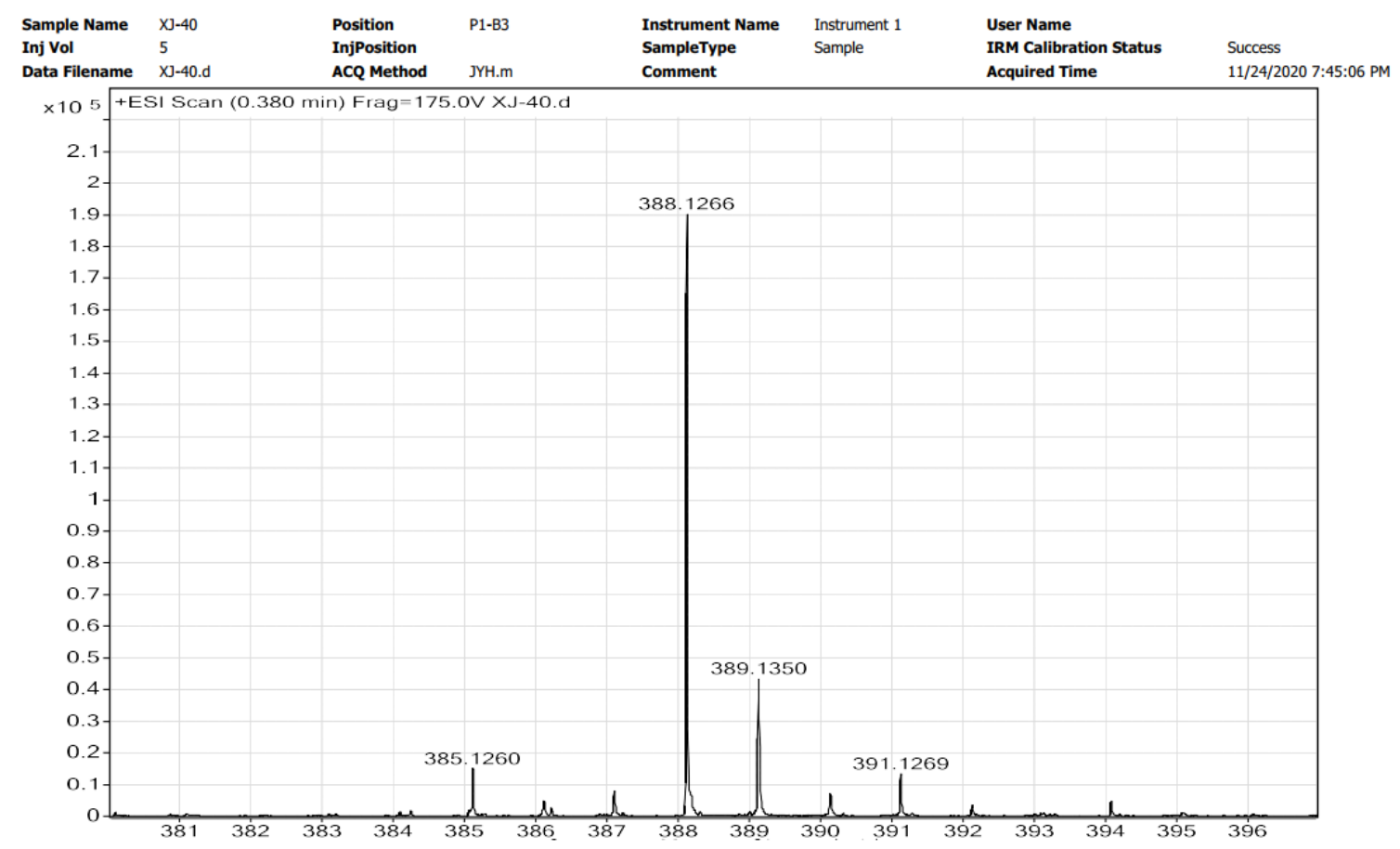

\begin{tabular}{|l|r|r|r|r|r|r|r|}
\hline Formula (M) & Score (MFG) & \multicolumn{1}{c|}{ Mass } & Mass (MFG) & $\mathrm{m} / \mathrm{z}($ Calc) & Diff (ppm) & DBE & $\mathrm{m} / \mathrm{z}$ \\
\hline C20 H19 N3 O4 & 99.91 & 365.1374 & 365.1376 & 388.1268 & 0.49 & 388.1266 \\
\hline
\end{tabular}


41

\begin{tabular}{|c|c|c|c|c|c|c|c|}
\hline $\begin{array}{l}\text { Sample Name } \\
\text { Inj Vol }\end{array}$ & $\begin{array}{l}X]-41-2 \\
5\end{array}$ & $\begin{array}{l}\text { Position } \\
\text { InjPosition }\end{array}$ & P1-B4 & $\begin{array}{l}\text { Instrument Name } \\
\text { SampleType }\end{array}$ & $\begin{array}{l}\text { Instrument } 1 \\
\text { Sample }\end{array}$ & $\begin{array}{l}\text { User Name } \\
\text { IRM Calibration Status }\end{array}$ & Success \\
\hline Data Filename & $\mathrm{xJ}-41-2 . \mathrm{d}$ & ACQ Method & JYH.m & Comment & & Acquired Time & $11 / 23 / 20202: 51: 37 \mathrm{PM}$ \\
\hline
\end{tabular}

$\times 105+$ +ESI Scan (0.078 min) Frag=175.0V $\times J-41-2 . d$

1.3 -

$1.25-$

1.2

1.15

1.05

$0.95-$

$0.9-1-8$
$0.85-10$

0.8

0.75

0.7
$0.65-1$

0.6

0.55

$0.5-1$
$0.45-1$

0.4

0.35

$0.3-1$
$0.25-1$

0.2

$0.15-$

\begin{tabular}{r|r}
0.15 \\
0.1
\end{tabular}$\quad 396.1544$

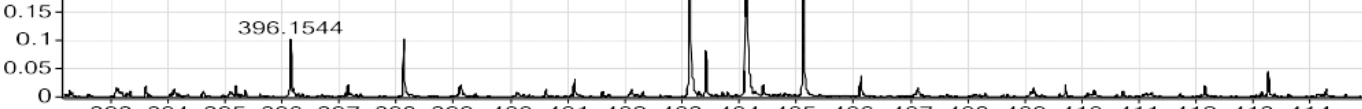

393394395396397398399400.401402403404 .405406407408409410411412413414

\begin{tabular}{|l|r|r|r|r|r|r|}
\hline Formula (M) & Score (MFG) & \multicolumn{1}{c|}{ Mass } & Mass (MFG) & m/z (Calc) & Diff (ppm) & DBE \\
\hline C20 H19 N3 O5 & 94.78 & 381.1339 & 381.1325 & 404.1217 & -3.69 & 404.1231 \\
\hline
\end{tabular}

42

\begin{tabular}{|c|c|c|c|c|c|c|c|}
\hline Sample Name & $\mathrm{XJ}-42$ & Position & P1-B1 & Instrument Name & Instrument 1 & User Name & \\
\hline Inj Vol & 5 & InjPosition & & SampleType & Sample & IRM Calibration Status & Success \\
\hline Data Filename & $\mathrm{xJ}-42 . \mathrm{d}$ & ACQ Method & JYH.m & Comment & & Acquired Time & 11/24/2020 8:14:18 PM \\
\hline
\end{tabular}

$\times 105+$ ESI Scan (1.181 min) Frag=175.0V $\times J-42 . d$

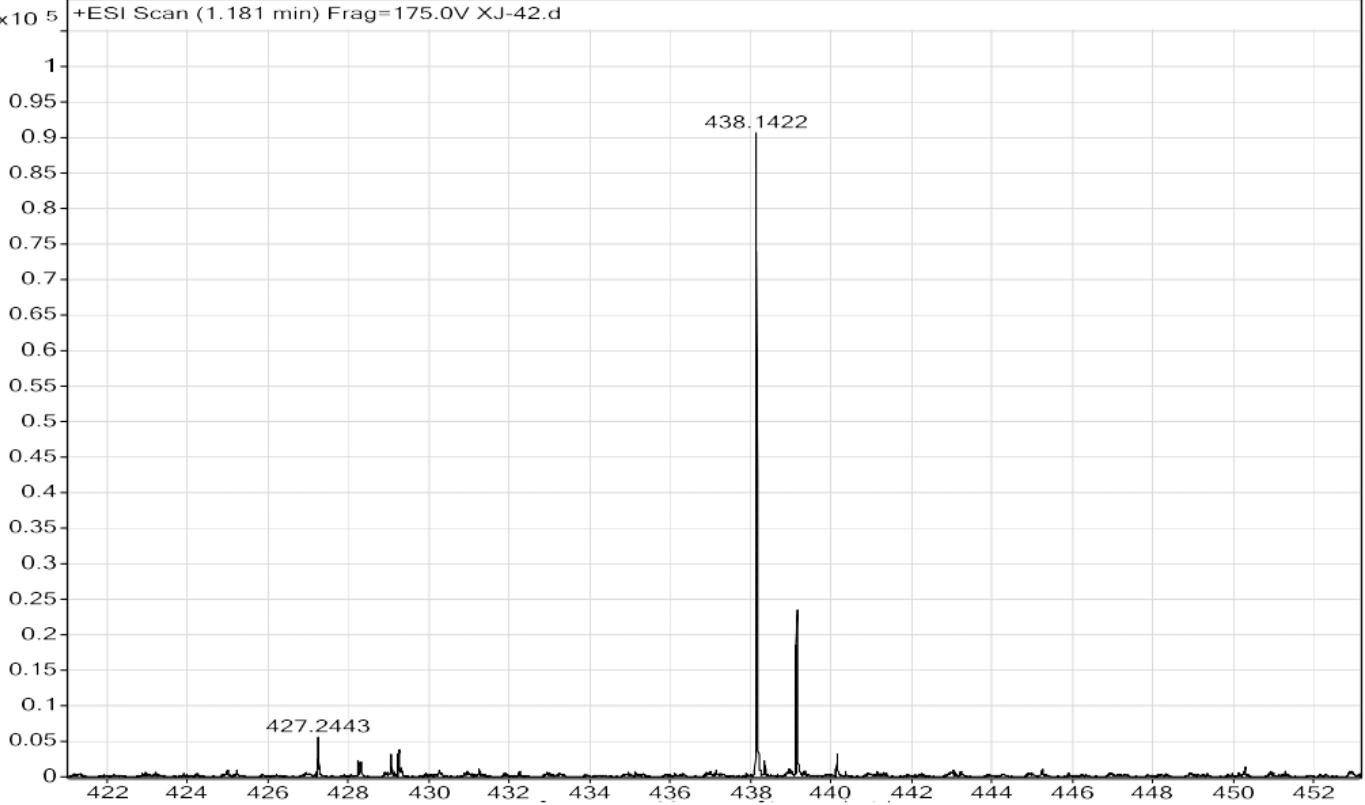

\begin{tabular}{|l|r|r|r|r|r|r|}
\hline Formula (M) & Score (MFG) & \multicolumn{1}{c|}{ Mass } & Mass (MFG) & m/z (Calc) & Diff (ppm) & DBE \\
\hline C24 H21 N3 O4 & 99.87 & 415.153 & 415.1532 & 438.1424 & 0.55 & 438.1422
\end{tabular}


43

\begin{tabular}{|c|c|c|c|c|c|c|c|}
\hline $\begin{array}{l}\text { Sample Name } \\
\text { Inj Vol }\end{array}$ & $\begin{array}{l}X J-43-2 \\
5\end{array}$ & $\begin{array}{l}\text { Position } \\
\text { InjPosition }\end{array}$ & P1-A6 & $\begin{array}{l}\text { Instrument Name } \\
\text { SampleType }\end{array}$ & $\begin{array}{l}\text { Instrument } 1 \\
\text { Sample }\end{array}$ & $\begin{array}{l}\text { User Name } \\
\text { IRM Calibration Status }\end{array}$ & Success \\
\hline Data Filename & $x]-43-2 . d$ & ACQ Method & JYH.m & Comment & & Acquired Time & 11/25/2020 7:47:53 PM \\
\hline
\end{tabular}

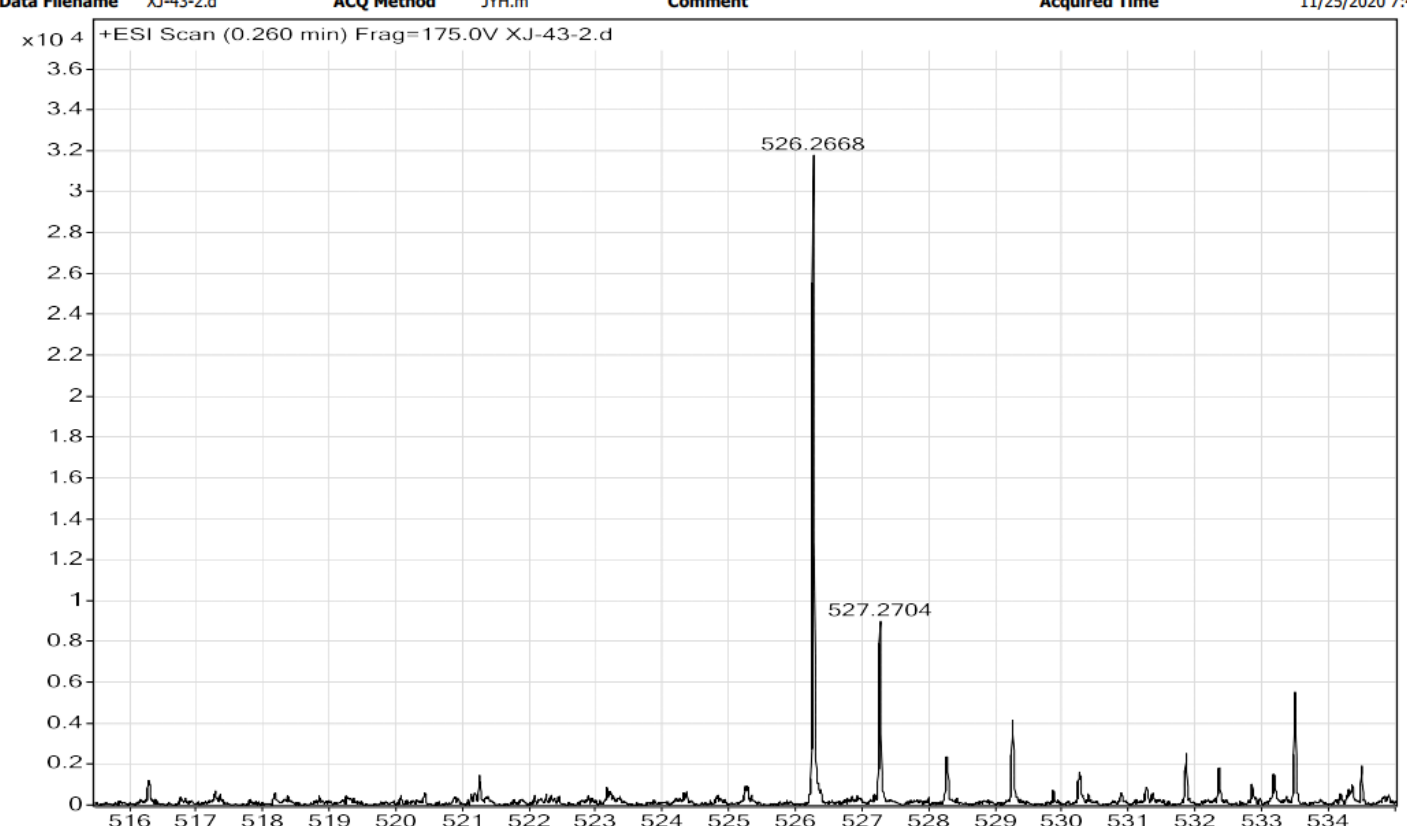

\begin{tabular}{|l|r|r|r|r|r|r|}
\hline Formula (M) & Score (MFG) & \multicolumn{1}{c|}{ Mass } & Mass (MFG) & m/z (Calc) & Diff (ppm) & DBE \\
\hline C30 H37 N3 O4 & 98.62 & 503.2776 & 503.2784 & 526.2676 & 1.64 & 526.2668 \\
\hline
\end{tabular}

45

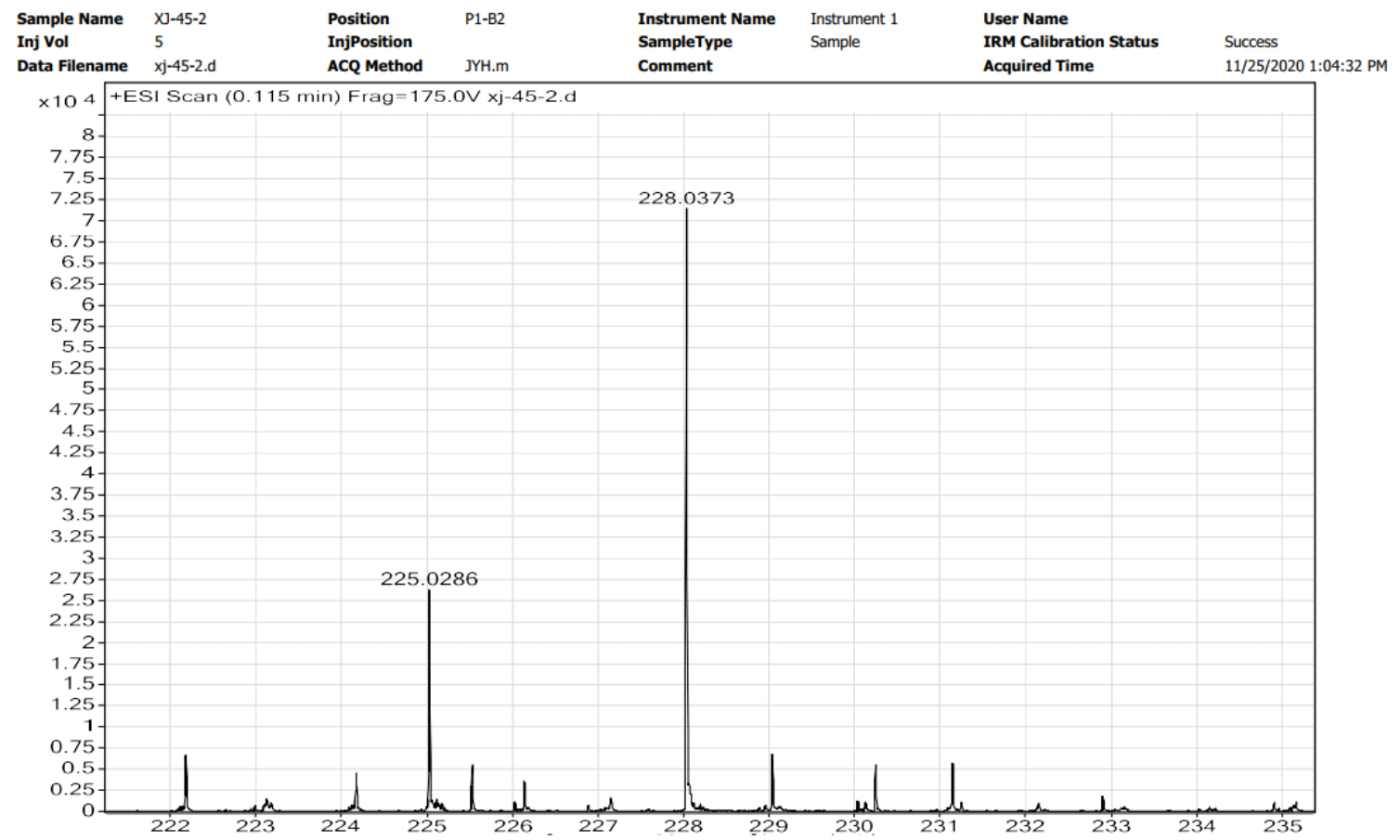

\begin{tabular}{|c|r|r|r|r|r|r|r|}
\hline Formula (M) & Score (MFG) & Mass & Mass (MFG) & $\mathrm{m} / \mathrm{z}$ (Calc) & Diff (ppm) & DBE & $\mathrm{m} / \mathrm{z}$ \\
\hline C9 H7 N3 O3 & 97.94 & 205.0481 & 205.0487 & 228.038 & 3.23 & 8 & 228.0373 \\
\hline
\end{tabular}


46

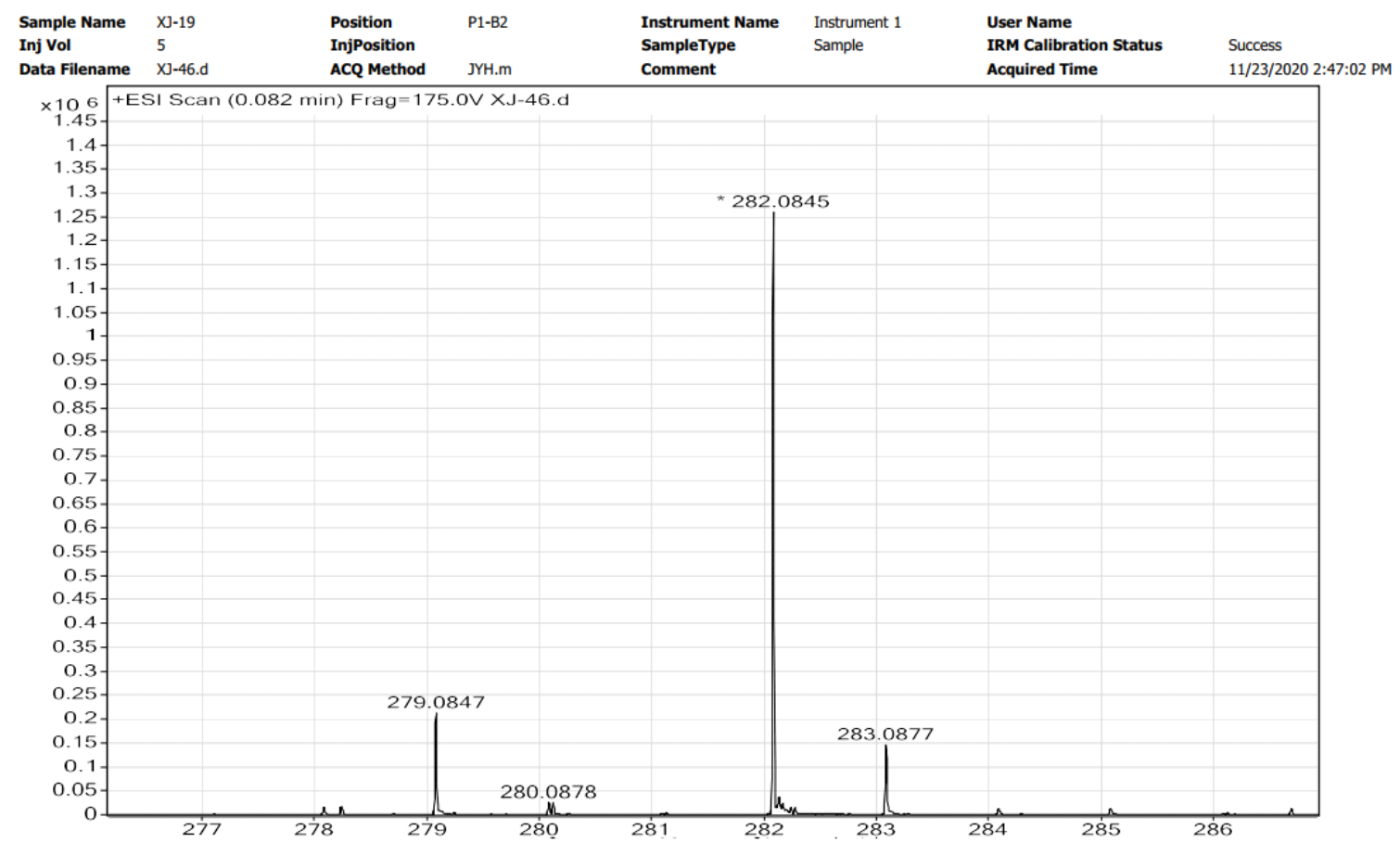

\begin{tabular}{|l|r|r|r|r|r|r|r|}
\hline Formula (M) & Score (MFG) & \multicolumn{1}{|c|}{ Mass } & Mass (MFG) & $\mathrm{m} / \mathrm{z}($ Calc) & Diff (ppm) & DBE & $\mathrm{m} / \mathrm{z}$ \\
\hline C13 H13 N3 O3 & 99.32 & 259.0953 & 259.0957 & 282.0849 & 1.59 & 9 & 282.0845 \\
\hline
\end{tabular}

48

\section{Mass Spectrum SmartFormula Report}

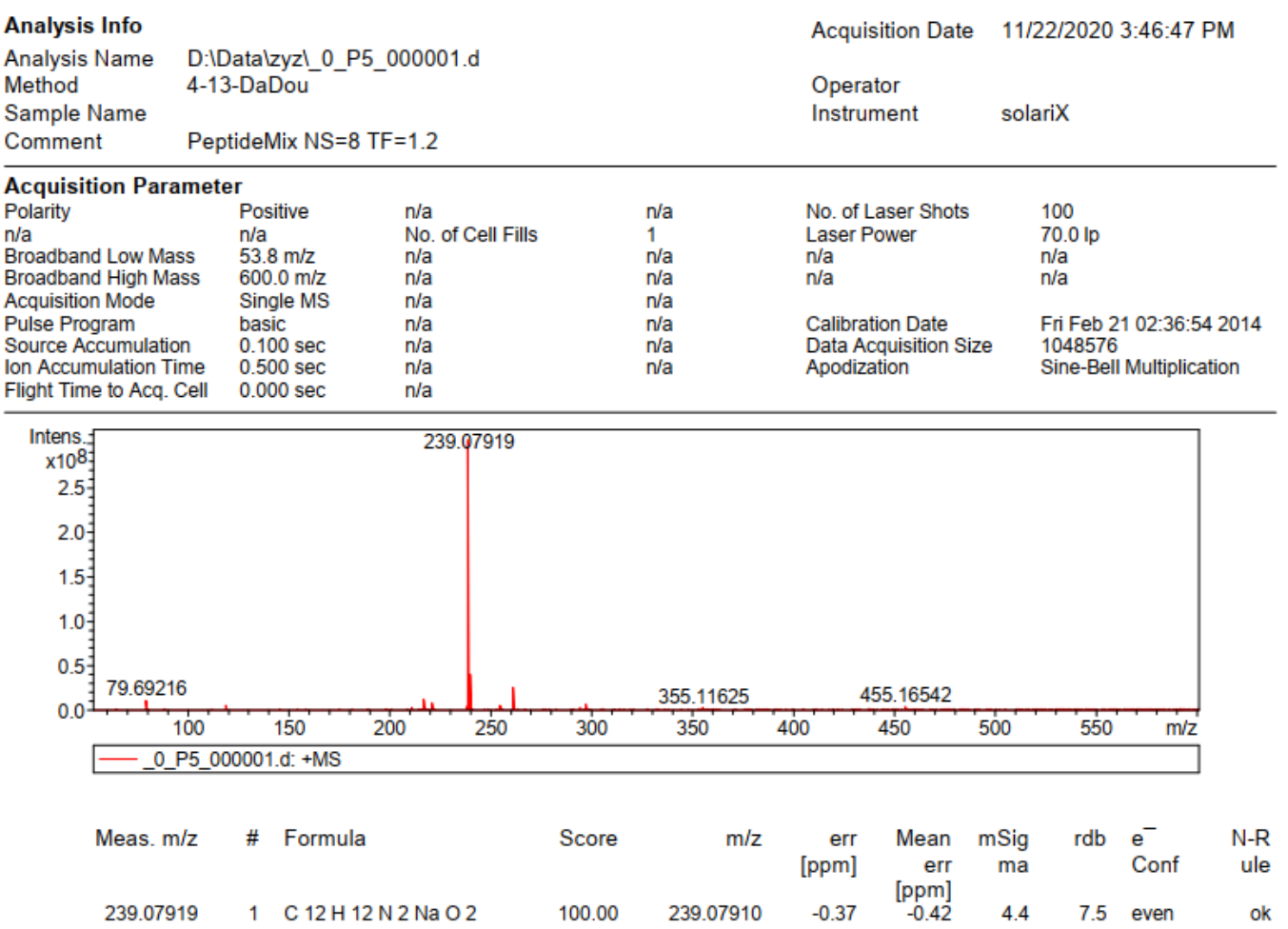


50

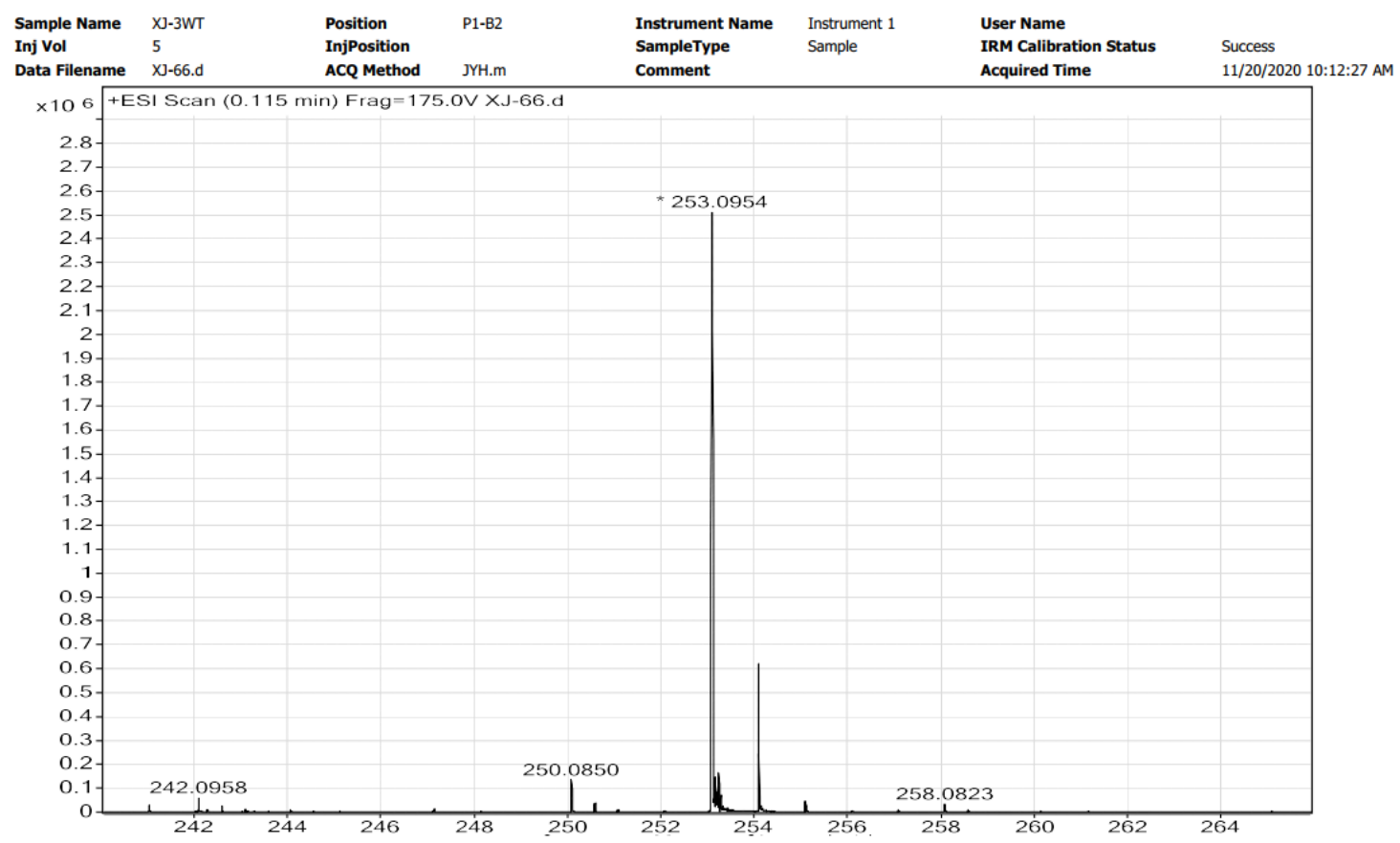

\begin{tabular}{|l|r|r|r|r|r|r|r|}
\hline Formula (M) & Score (MFG) & \multicolumn{1}{c|}{ Mass } & Mass (MFG) & $\mathrm{m} / \mathrm{z}($ Calc) & Diff (ppm) & DBE & $\mathrm{m} / \mathrm{z}$ \\
\hline $\mathrm{C} 13 \mathrm{H} 14 \mathrm{~N} 2 \mathrm{O} 2$ & 98.17 & 230.1062 & 230.1055 & 253.0947 & -2.83 & 8 & 253.0954 \\
\hline
\end{tabular}

\section{1}

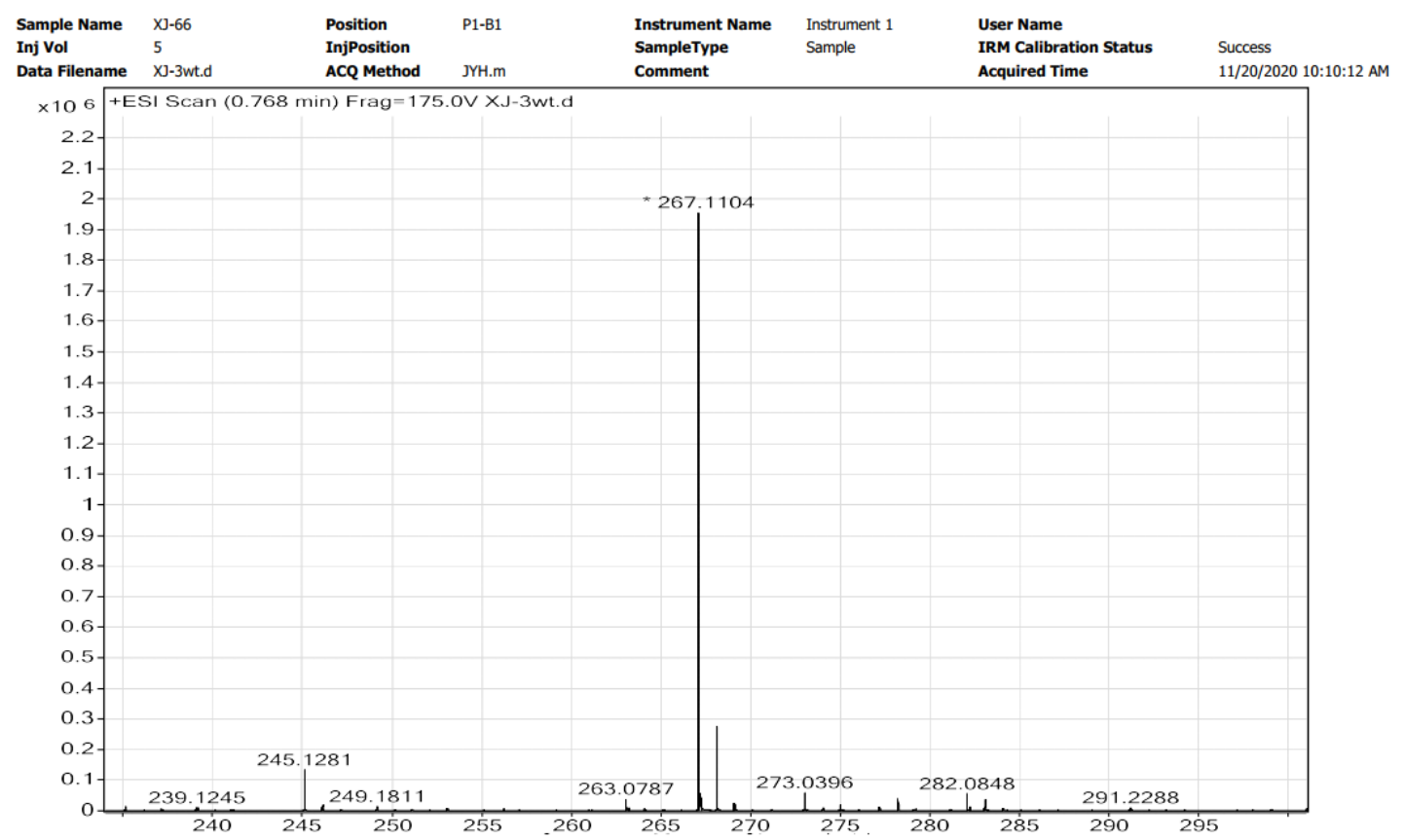

\begin{tabular}{|l|r|r|r|r|r|r|r|}
\hline Formula (M) & Score (MFG) & Mass & Mass (MFG) & $\mathrm{m} / \mathrm{z}$ (Calc) & Diff (ppm) & DBE & $\mathrm{m} / \mathrm{z}$ \\
\hline C14 H16 N2 O2 & 100 & 244.1212 & 244.1212 & 267.1104 & 0 & 8 & 267.1104 \\
\hline
\end{tabular}

